\title{
IntechOpen
}

\section{Voice and Swallowing Disorders}

Edited by Monjur Ahmed 



\section{Voice and Swallowing Disorders}

Edited by Monjur Ahmed 

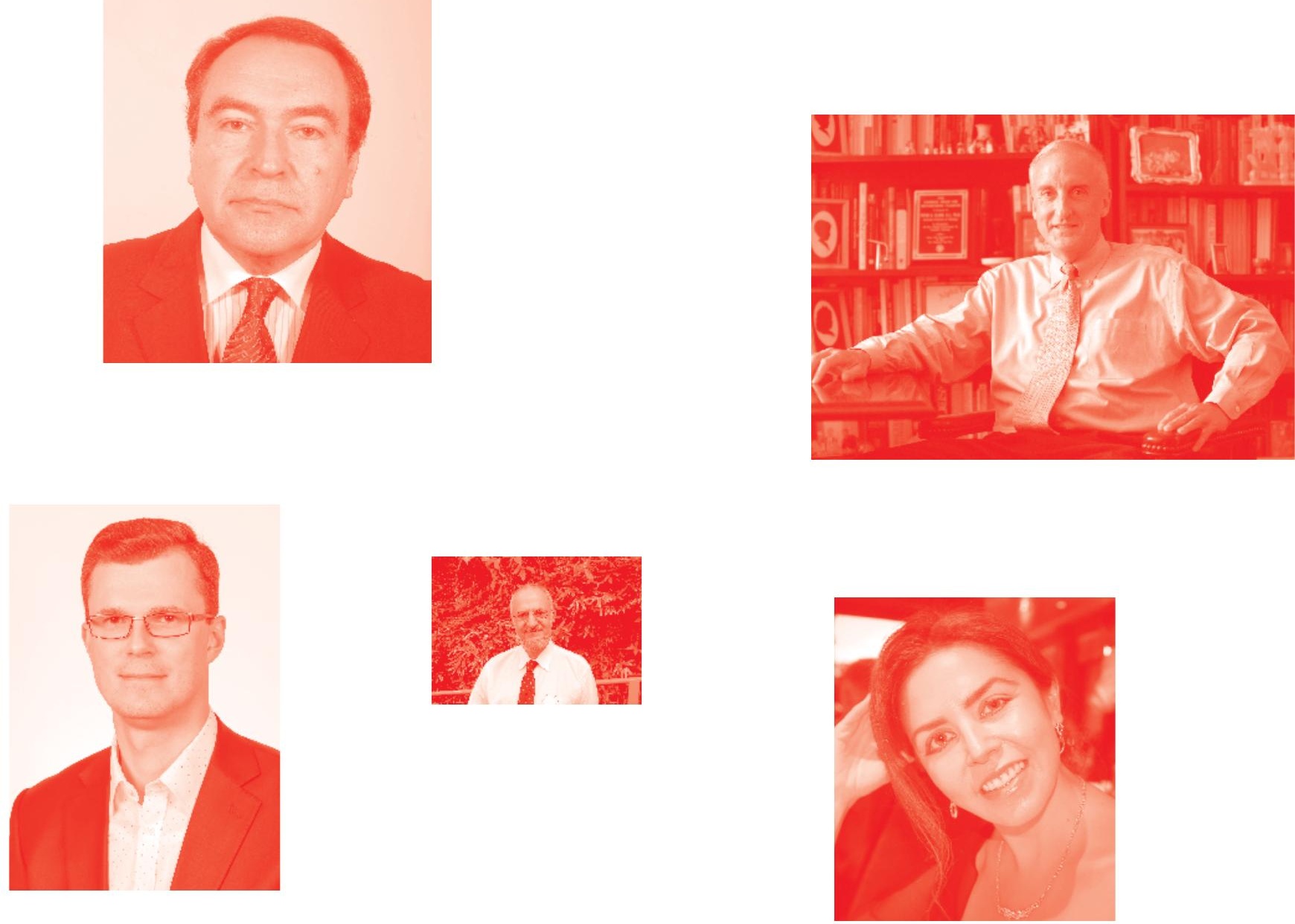

Supporting open minds since 2005
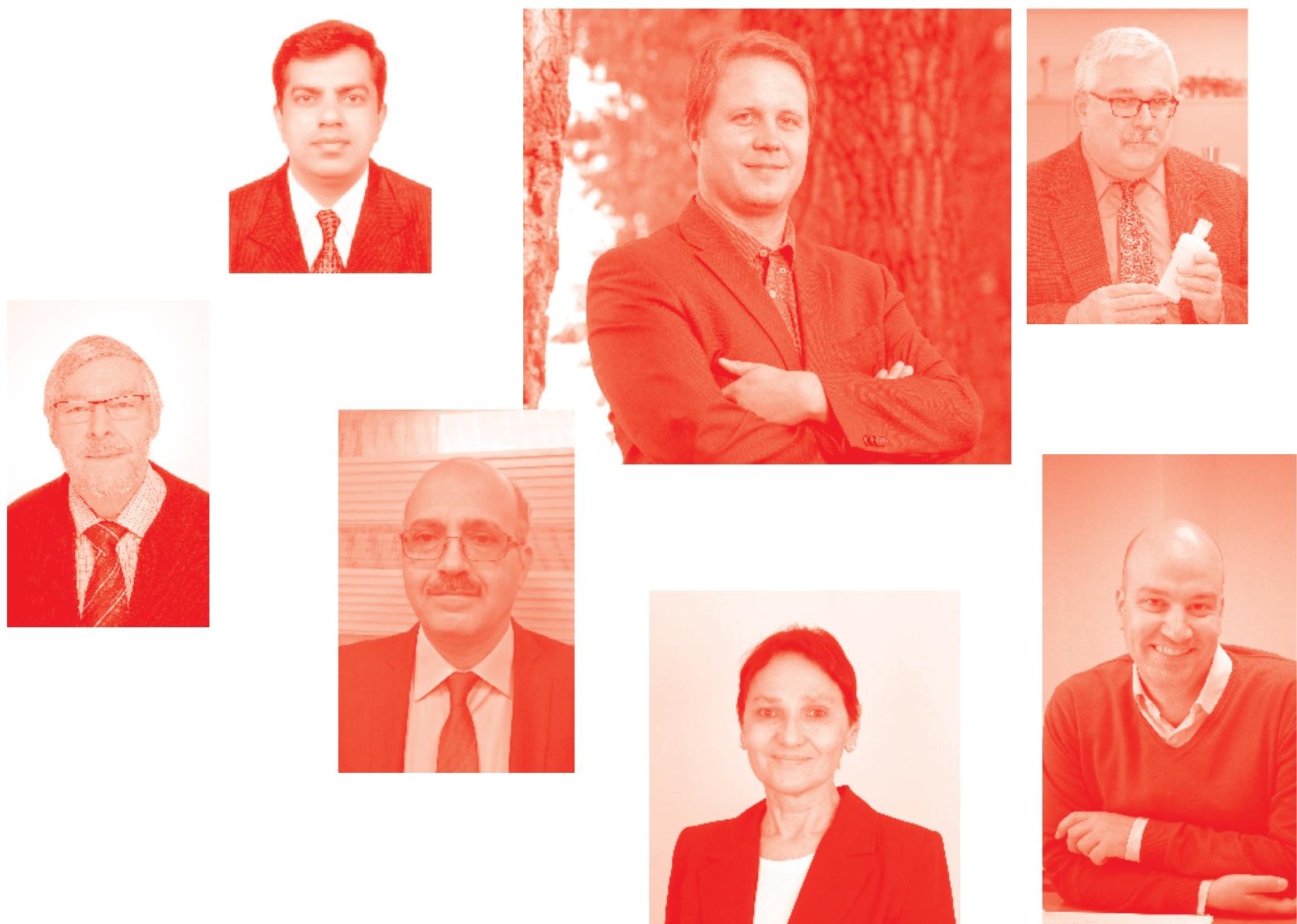
Voice and Swallowing Disorders

http: //dx. doi.org/10.5772/intechopen. 77662

Edited by Monjur Ahmed

\section{Contributors}

Yozo Miyaoka, Nil Çomunoğlu, Şebnem Batur, Mine Önenerk, Shinichiro Maeshima, Aiko Osawa, Mohammed Basha, Stefano Ursino, Fabiola Paiar, Paola Cocuzza, Stefania Santopadre, Bruno Fattori, Yumi Tsunoda, Hisao Hiraba, Daniela Vrinceanu, Mihai Dumitru

( ) The Editor(s) and the Author(s) 2020

The rights of the editor(s) and the author(s) have been asserted in accordance with the Copyright, Designs and Patents Act 1988. All rights to the book as a whole are reserved by INTECHOPEN LIMITED. The book as a whole (compilation) cannot be reproduced, distributed or used for commercial or non-commercial purposes without INTECHOPEN LIMITED's written permission. Enquiries concerning the use of the book should be directed to INTECHOPEN LIMITED rights and permissions department (permissions@intechopen.com).

Violations are liable to prosecution under the governing Copyright Law .

\section{(cc) BY}

Individual chapters of this publication are distributed under the terms of the Creative Commons Attribution 3.๑ Unported License which permits commercial use, distribution and reproduction of the individual chapters, provided the original author(s) and source publication are appropriately acknowledged. If so indicated, certain images may not be included under the Creative Commons license. In such cases users will need to obtain permission from the license holder to reproduce the material. More details and guidelines concerning content reuse and adaptation can be found at http : //www . intechopen . com/copyright-policy . html .

\section{Notice}

Statements and opinions expressed in the chapters are these of the individual contributors and not necessarily those of the editors or publisher. No responsibility is accepted for the accuracy of information contained in the published chapters. The publisher assumes no responsibility for any damage or injury to persons or property arising out of the use of any materials, instructions, methods or ideas contained in the book.

First published in London, United Kingdom, 2020 by IntechOpen IntechOpen is the global imprint of INTECHOPEN LIMITED, registered in England and Wales, registration number: 11086078 , 7th floor, 10 Lower Thames Street, London,

EC3R 6AF, United Kingdom

Printed in Croatia

British Library Cataloguing-in-Publication Data

A catalogue record for this book is available from the British Library

Additional hard and PDF copies can be obtained from orders@intechopen.com

Voice and Swallowing Disorders

Edited by Monjur Ahmed

p. $\mathrm{cm}$.

Print ISBN 978-1-83880-365- 0

Online ISBN 978-1-83880-366-7

eBook (PDF) ISBN 978-1-83880-105-2 


\section{We are IntechOpen, \\ the world's leading publisher of Open Access books}

Built by scientists, for scientists

\section{$4,700+$}

Open access books available

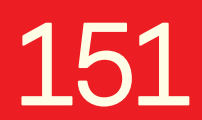

Countries delivered to
$120,000+$

International authors and editors

Our authors are among the

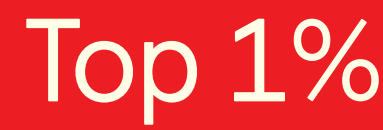

most cited scientists

Contributors from top 500 universities
$135 \mathrm{M}+$

Downloads

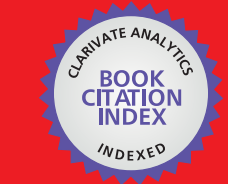

WEB OF SCIENCE ${ }^{\mathrm{MM}}$

Selection of our books indexed in the Book Citation Index in Web of Science ${ }^{\mathrm{TM}}$ Core Collection (BKCI)

\section{Interested in publishing with us? \\ Contact book.department@intechopen.com}

Numbers displayed above are based on latest data collected.

For more information visit www.intechopen.com 



\section{Meet the editor}

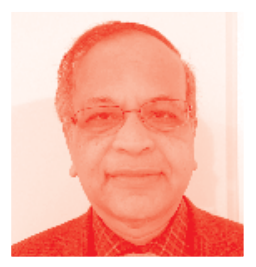

Monjur Ahmed, MD, FRCP is working as an Associate Professor of Medicine at Thomas Jefferson University, Philadelphia, Pennsylvania, USA. He has been a practicing gastroenterologist for 21 years. His special interests are in eosinophilic esophagitis, gastrointestinal motility, and dysphagia. He also serves as an Editor-in-Chief of the World Journal of Gastrointestinal Oncology. 



\section{Contents}

Preface

Section 1

Physiological Aspect of Dysphagia

Chapter 1

Quantitative Analysis of Activity Patterns in the Muscles of

Mastication and Deglutition

by Yozo Miyaoka

Section 2

Pathological Aspect of Dysphagia

Chapter 2

Pathology of Nonneoplastic Lesions of the Vocal Folds

by Nil Çomunoğlu, Şebnem Batur and Ayşe Mine Önenerk

\section{Section 3}

Dysphagia in Stroke Patients

Chapter 3

Swallowing Disorders in Patients with Stroke

by Aiko Osawa and Shinichiro Maeshima

Section 4

Maxillofacial and Oral Dysphagia

Chapter 4

Maxillofacial and Oral Aspects of Dysphagia

by Mohammed Basha

Section 5

Radiation-Related Dysphagia

Chapter 5

Radiation-Related Dysphagia: From Pathophysiology to

Clinical Aspects

by Stefano Ursino, Paola Cocuzza, Stefania Santopadre, Fabiola Paiar and Bruno Fattori 
Section 6

Salivation by Somatosensory Stimulation

Chapter 6

Effect of Salivation by Facial Somatosensory Stimuli of Facial

Massage and Vibrotactile Apparatus

by Tsunoda Yumi, Akatuka Sumiko, Fukui Sayaka, Nakayama Enri, Abe Kimiko, Sato Mituyasu, Kimura Masanori, Kato Syunnichiryou, Sakai Maho, Yamaoka Masaru, Watanabe Mao, Ueda Koichirou and Hiraba Hisao

Section 7

Dysphagia Due to Cervical and Facial Tumors

Chapter 7

Swallowing Disorders in Cervical Facial Tumors

by Daniela Vrinceanu and Mihai Dumitru 


\section{Preface}

This book is an excellent source of information on voice and swallowing disorders. It describes not only the physiologic and pathologic aspects of various voice and swallowing disorders but also the differential diagnoses and clinical management of these disorders. The chapter on voice changes due to various non-neoplastic lesions of vocal cord is very interesting. As I was editing this book, I thought that certain chapters were of paramount importance in clinical practice, particularly oropharyngeal dysphagia, dysphagia due to cervical facial tumors, radiationinduced dysphagia, and dysphagia in patients with a stroke. This book can serve as an invaluable reference to practicing gastroenterologists, oral surgeons, and ear, nose, and throat surgeons.

Monjur Ahmed

Thomas Jefferson University,

Philadelphia, 

Section 1

\section{Physiological Aspect of Dysphagia}





\title{
Chapter 1
}

\section{Quantitative Analysis of Activity Patterns in the Muscles of Mastication and Deglutition}

\author{
Yozo Miyaoka
}

\begin{abstract}
Surface electromyograms (EMGs) were recorded from the masseter (Mass), one of the major muscles for chewing, and from the suprahyoid ( $\mathrm{SH}$ ) muscles, involved in swallowing. Activity patterns of these EMGs were analyzed with a $T_{P}$ method that was developed specifically to quantify muscle activity patterns. To compare individual EMG bursts in a participant with different amplitudes and active durations, the bursts were cumulatively integrated to standardize the amplitudes and active durations. Each $T_{P}$ value calculated by this method indicated a relative location of an EMG burst on a standardized time scale free from changes in the amplitudes and active durations. Both $\operatorname{In}_{P}$ and $D_{P}$ values were derived from the $T_{P}$ values and also applied to the burst. $\mathrm{A} \mathrm{T}_{50}$ value indicated the standardized time for half of the final cumulatively integrated EMG burst. Five groups of application samples were introduced to demonstrate the usefulness of the $\mathrm{T}_{\mathrm{P}}$ method in comparing activity patterns of the Mass and SH EMGs during chewing and swallowing, while participants were in different body positions and experiencing different tastes and textures of sample foods. Finally, limitations and perspectives of the $T_{P}$ method are discussed.
\end{abstract}

Keywords: electromyogram, activity pattern, masseter, suprahyoid, quantification

\section{Introduction}

More than two decades ago, a simple but fundamental research question about evaluation of EMG data arose. Shouldn't two or more EMG bursts with similar amplitudes and duration of activity but visibly different activity patterns be expected to encode different functional meanings? At that time, however, such EMG bursts were generally regarded as the same from a quantitative aspect, namely, the activity patterns were ignored in the evaluation. I realized that the situation was mainly caused by the lack of a suitable and specific parameter for the evaluation, although some researchers used the term, activity patterns, differently or (maybe) inappropriately. This is the reason why I intended to newly develop a parameter for quantitative evaluation of activity patterns of EMGs, which is introduced in this chapter.

\subsection{Neural basis of mastication and deglutition}

Mastication is one of the movements essential for the survival of mammals, including humans, and the reciprocal activities of the jaw-closing and 
jaw-opening muscles are among the prominent features of the movement. The Mass, innervated by motoneurons of the trigeminal nerve and one of the major jaw-closing muscles, works to crush and grind the food ingested into the mouth during natural mastication. The digastric muscle is composed of the anterior and posterior bellies that are innervated by motoneurons of the trigeminal and facial nerves, respectively. This muscle is one of the $\mathrm{SH}$ muscles that are part of the major jaw-opening muscles. Amplitudes and active durations of the jaw-closing and jaw-opening muscles change moment by moment both reflexively and voluntarily in accordance with physical and chemical conditions of the chewing food (bolus) in the mouth [1]. Mastication is performed not only by the jaw but also by the tongue and its related muscles as well. Basically, the jaw-closing muscles are coordinated with the tongue retractors during natural chewing, whereas the jaw-opening muscles are coordinated with the tongue protractors [1]. These and other tongue muscles - all but the palatoglossus - are innervated by the XII motoneurons [2].

Deglutition followed by mastication is also one of the essential movements for survival. A previous study [3] revealed that healthy Japanese adults swallow, on average, 585 (range, 203 to 1008) times a day. Swallowing is a complicated and coordinated movement performed by approximately 22 muscles [4]. The SH muscles are innervated by three pairs of cranial nerves (V, VII, and XII) and are composed of eight muscles: digastric, geniohyoid, genioglossus, hyoglossus, mylohyoid, palatoglossus, styloglossus, and stylohyoid. Conventionally, the sequence of deglutition is classified as the oral, pharyngeal, and esophageal stages. The SH muscles are among the major components of the muscles especially related to the pharyngeal stage of deglutition. Pharyngeal swallowing is a highly automated or a kind of "stereotyped" movement [5]; therefore, once the movement begins, a series of muscle excitations should occur at a fixed order. However, swallowing is affected by subjective factors, such as age, and by objective factors, such as volume of foods [6].

The sensory systems in the oropharyngeal and laryngeal regions provide critical cues for reflexive and voluntary regulations of masticatory and deglutition movements. These regions have four groups of sensory receptors: mechanoreceptors, thermal (temperature) receptors, chemical receptors, and nociceptors [5]. All except nociceptors are involved in the processes discussed in this chapter. Each of these three sensory receptors has different transduction mechanisms, but once they are excited by sensory stimuli, identical action potentials are conveyed from these sensory receptors to the central nervous system through four pairs of cranial nerves: trigeminal (V), facial (VII), glossopharyngeal (IX), and vagus (X) nerves [5]. The chorda tympani nerve is a sensory (taste) branch of the facial nerve, and the superior laryngeal nerve is a sensory branch of the vagus nerve. Many previous studies have documented that the chemoreceptors in the oropharyngeal and laryngeal regions and the neurons in the central nervous system driven by the chemoreceptors often respond not only to taste but also to mechanical or thermal stimuli or both (i.e., multiple sensitivity of receptors and neurons). For example, an electrophysiological study in rats showed that $62.3 \%$ of 169 taste neurons in the nucleus of the solitary tract (the primary taste nucleus), which connected with the neurons in the parabrachial nucleus (the secondary taste nucleus), were excited by mechanical or thermal stimuli (or both) as well as by chemical (taste) stimuli applied to the oral region [7]. This multiple sensitivity is characteristic of the chorda tympani [8], parabrachial nucleus [9], thalamic [10], and cortical neurons [11]. 


\subsection{Necessity of a new parameter for activity pattern}

A chewing sequence is composed of a series of several bursts of masseteric activities, and each burst usually has a different amplitude and active duration. Figure 1 depicts sample data of masseteric activity, which were recorded during the chewing of a pineapple-flavored gummy candy (GC) by a healthy young adult. The raw masseteric EMG is elicited by contraction of the Mass, and the height of the integrated EMG reflects firing rates of the muscle. The raw masseteric EMG bursts appear periodically during the jaw-closing phase and are silent during the jaw-opening phase, although the active duration of masseteric activity does not always exactly match with the jaw-closing phase [1]. The firing rates of the masseteric EMG in Figure 1 gradually increased, reached a peak of approximately one every $0.3 \mathrm{~s}$ after the beginning of firing, and diminished rather rapidly; therefore, the individual raw masseteric EMG recordings looked like spindles. Moreover, the integrated masseteric EMG shows that the height of the peaks, as well as the length of active duration, differed among the three bursts: The peak height of the integrated EMG of the third burst is 33\% larger than that of the first, whereas the active duration of the third burst was $42 \%$ shorter than the first.

The term "pattern" is somewhat confusing because changes in amplitudes and active durations of muscle activity are often difficult to interpret. In the majority of previous studies, the term reflects a more comprehensive concept than in my study. For example, in a previous study [12], the authors recorded masseteric activity during the chewing of various foods. The behavior was classified into five subgroups with a cluster analysis technique, mainly by chewing time and muscle work rate. Masseteric EMGs of the same activity (analogous masseteric EMGs) with different amplitudes and active durations must have similar appearances or the same changes in percentages, but I believe that analogous bursts should be regarded as having the same pattern. In this [12] and other studies [13-15], the authors evaluated the patterns of masticatory muscle activity according to the amplitude and duration parameters or their derivatives

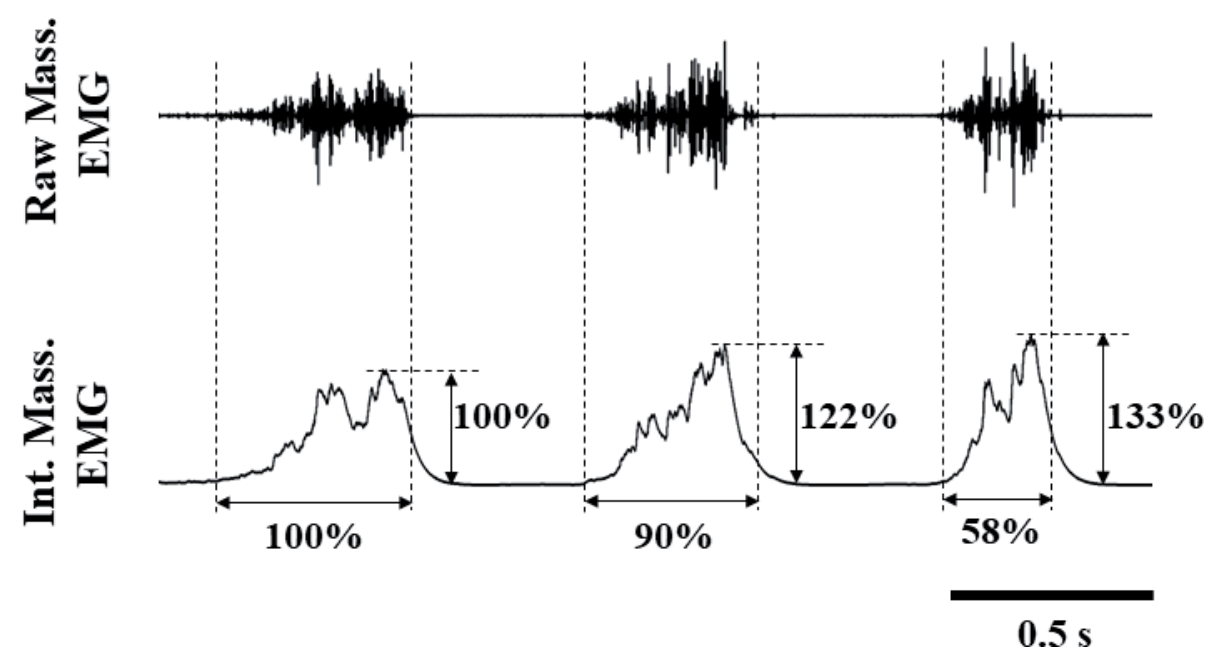

Figure 1.

Sample data of raw and integrated Mass activity during chewing of a gummy candy. Three successive bursts in an electromyogram of the Mass, recorded during natural chewing of a pineapple-flavored gummy candy. Percentages in amplitudes and active durations of the second and third bursts are shown in reference to those of the first burst. Vertical dotted lines indicate the beginnings and ends of activity (bursts) of the electromyograms. Scales for the vertical axis are omitted for clarity. Raw Mass. EMG: raw masseteric electromyogram; Int. Mass. EMG: integrated masseteric electromyogram. 
for the individual sequences. In contrast to these previous studies, my colleagues and I used specific parameters, $\mathrm{T}_{50}$ and $\mathrm{D}_{50}$ values (described later), to evaluate individual chewing bursts in standardized amplitude and duration scales. It is necessary to standardize both the amplitude and active duration to avoid such confusion and to compare their pure activity patterns. According to our method, analogous EMG bursts are judged to have the same activity patterns even if there are large differences in amplitudes, active durations, and derivatives among the EMG bursts.

Figure 2 further clarifies the reason why a specific parameter is needed to analyze activity patterns. The masseteric EMG on the left side was recorded during chewing, and the EMG on the right side was digitally produced by reversing the time sequence of the EMG on the left side. Consequently, both heights and active durations of the EMGs should be completely identical because the original data were the same. However, this figure shows that the masseteric activity is weaker during the initial stage and stronger during the last stage on the left side than on the right side. In the other words, these EMGs show different activity patterns: The left one shows a firing pattern of increments, and the right one shows a firing pattern of decrements. According to the $\mathrm{T}_{\mathrm{P}}$ method (see "Development and verification of a $\mathrm{T}_{\mathrm{P}}$ method" section), these two firing patterns are quantified as clearly different values: For the incrementing firing pattern, the $T_{50}$ value is 0.579 , whereas for the decrementing pattern, the $T_{50}$ value is 0.423 . These results suggest that the strength of masseteric contraction depends on the stages (e.g., initial, middle, and last) of the chewing burst. Masseter contraction during natural chewing, not clenching, generates force to break down the food between the teeth. Therefore, chewing of different sample foods can alter firing sequences of the Mass. It is very difficult to appropriately interpret the sequences only by the changes in amplitudes and active durations of the Mass. This is why a specific parameter, $T_{P}$ value, is needed to evaluate muscle activity patterns; $\mathrm{T}_{\mathrm{P}}$ values can reveal differences in the activity patterns of individual bursts among various foods.

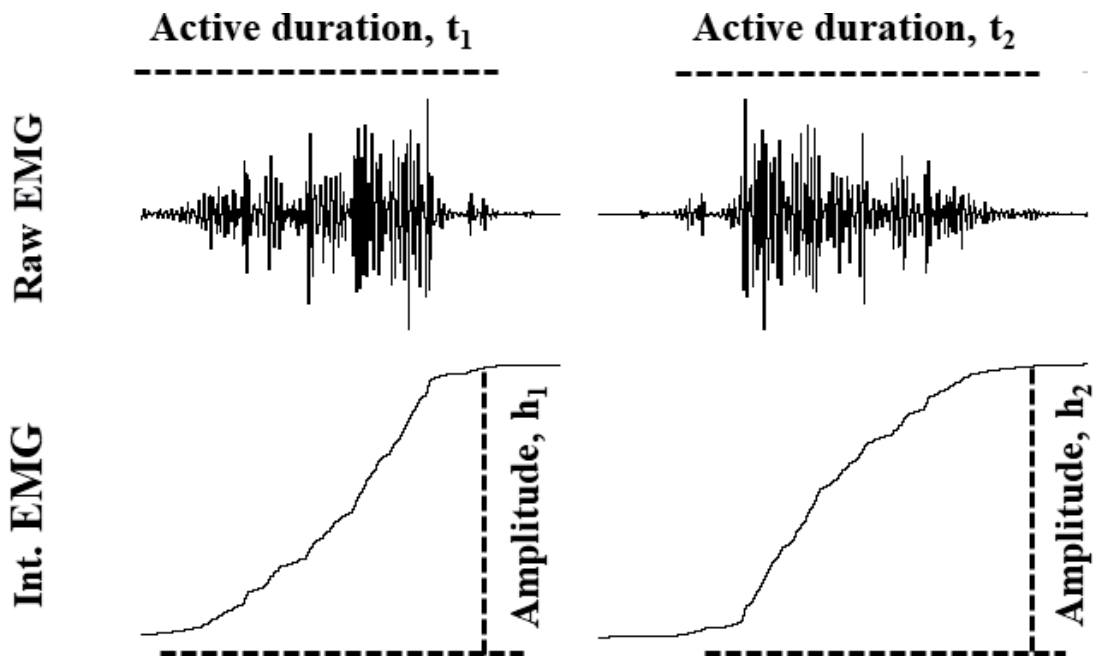

Figure 2.

Comparison of two EMGs with identical amplitude and active duration. The EMG on the left is the same as the second one in Figure 1, whereas the EMG on the right was produced mathematically on the basis of the left side. The left EMG has a pattern of increments, whereas the right EMG has a pattern of decrements. Horizontal dotted lines indicate the beginnings and the ends of activity (bursts) of the EMGs. Scales for the horizontal and vertical axes are omitted for clarity. Raw EMG: raw electromyogram; Int. EMG: integrated electromyogram. 


\section{Development and verification of a $T_{P}$ method}

\subsection{Development of a $T_{P}$ method}

The outlines of the standardization and calculation of $\mathrm{T}_{\mathrm{P}}$ values are as follows (Figure 3): (1) Individual active durations of muscle bursts of an EMG were set to 1.0 to standardize the durations; (2) the EMGs were cumulatively integrated from the beginning to the end of the duration; (3) the final value of the cumulative integration was set at $100 \%$; (4) the $100 \%$ value was divided into 4,5 , or 10 equal sections (i.e., each section contained $25 \%, 20 \%$, or $10 \%$ of the final value, respectively); (5) the standardized durations corresponding to individual $25 \%, 20 \%$, or $10 \%$ activity sections were determined; and (6) the intervals for the individual sections were successively named in the $T_{P}$ format (from $T_{25}$ to $T_{100}$, from $T_{20}$ to $\mathrm{T}_{100}$, or from $\mathrm{T}_{10}$ to $\mathrm{T}_{100}$, respectively). The use of any $\mathrm{T}_{\mathrm{P}}$ value basically depended on the precision of data analysis: $\mathrm{A}_{10}$ series was more suitable for a more precise analysis of activity patterns than were the $\mathrm{T}_{20}$ and $\mathrm{T}_{25}$ series. According to this simple method, each $\mathrm{T}_{\mathrm{P}}$ value indicated a relative location of the EMG on a standardized time scale that was free of changes in amplitudes and active durations; for example, $\mathrm{a}_{50}$ value indicated the standardized time for half of the final cumulative integrated EMG, and the $\mathrm{T}_{100}$ value was, by definition, always 1.0.

The $T_{P}$ method was applied to the data in Figure 1, and three $T_{P}\left(T_{20}, T_{50}\right.$, and $\mathrm{T}_{80}$ ) values were calculated for the data (Figure 3 ). In this example, a $\mathrm{T}_{50}$ value is used instead of $\mathrm{T}_{40}$ and $\mathrm{T}_{60}$ values just for the sake of convenience. If the Mass constantly fired during chewing through the active duration, then the three $\mathrm{T}_{\mathrm{P}}$ values should be 0.200 (for $\mathrm{T}_{20}$ ), 0.500 (for $\mathrm{T}_{50}$ ), and 0.800 (for $\mathrm{T}_{80}$ ) according to the definition of the $\mathrm{T}_{\mathrm{P}}$ method. In general, lower $\mathrm{T}_{\mathrm{P}}$ values indicated stronger firing than the constant firing, although larger $\mathrm{T}_{\mathrm{P}}$ values indicate weaker firing, as
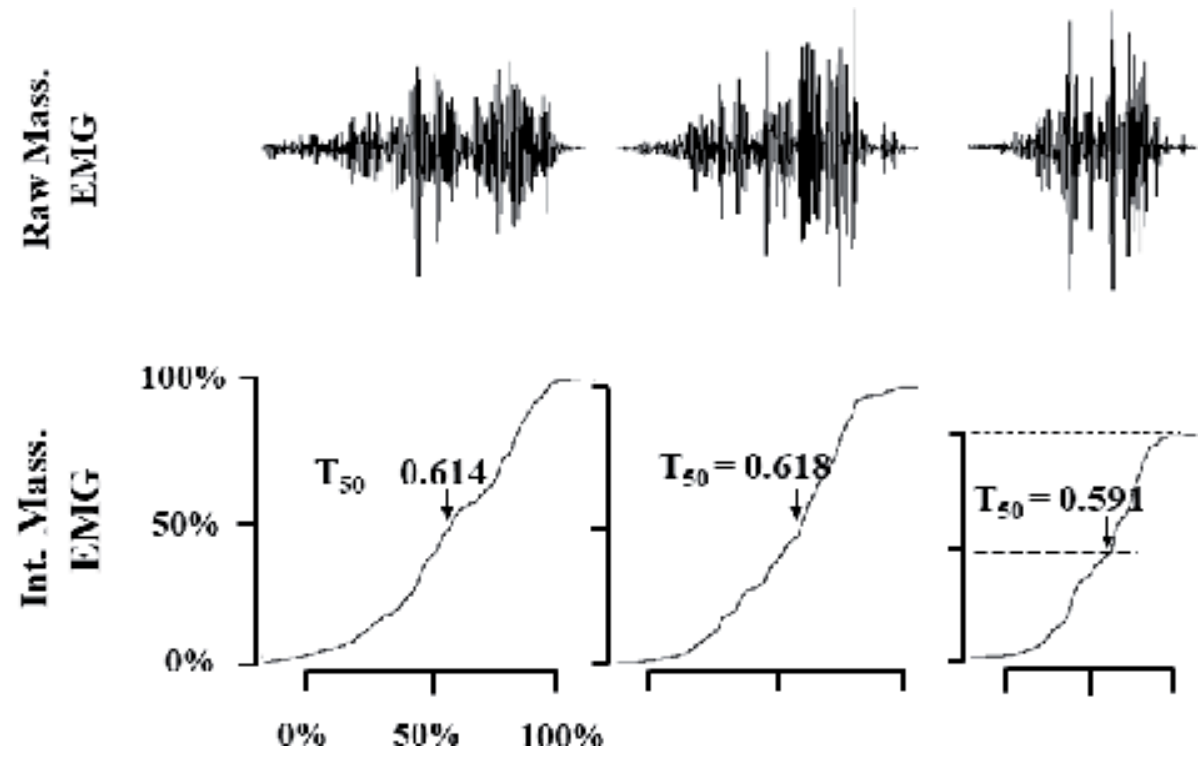

Figure 3.

Calculation of $T_{P}$ values from raw and cumulative Mass. EMG. The raw masseteric EMGs are identical to those in Figure 1. The three T20 values of those EMGs are 0.436 (for first burst), 0.382 (for second burst), and 0.369 (for third burst); the three T80 values are 0.827, 0.752 and 0.713 , respectively. Each lower horizontal dashed line indicates 50\%, and each horizontal dotted line indicates 100\%, of the integrated masseteric EMGs. Real scales for the horizontal and vertical axes are omitted for clarity. Raw Mass. EMG: raw masseteric electromyogram; Int. Mass. EMG: integrated masseteric electromyogram. 
described later [16]. Accordingly, the calculated three $\mathrm{T}_{50}$ values $(0.614,0.618$, and 0.591 ) in Figure 3 suggest that the activity pattern of the first burst is similar to that of the second one but not to that of the third one. However, some people may disagree with this suggestion of the $\mathrm{T}_{50}$ values. Contrary to the $\mathrm{T}_{50}$ values, the three $\mathrm{T}_{20}$ values calculated-0.436, 0.382 , and 0.369 - suggest that the activity pattern of the second burst is similar to that of the third one but not to that of the first one. Furthermore, the three $\mathrm{T}_{80}$ values calculated-0.827, 0.752, and 0.713-suggest that the activity patterns differ among the three bursts. Thus, sometimes at least three $\mathrm{T}_{\mathrm{P}}$ values (e.g., $\mathrm{T}_{20}, \mathrm{~T}_{50}$, and $\mathrm{T}_{80}$ ) may be needed in order to understand activity patterns appropriately.

\subsection{Verification of the $T_{P}$ method}

The $\mathrm{T}_{\mathrm{P}}$ method was verified mathematically by a computer simulation, in which the inverse Gaussian distribution was used. In the computer simulation, three EMG models $\left(\right.$ pro $_{\mathrm{A}}$, pro $_{\mathrm{B}}$, and pro $\left._{\mathrm{C}}\right)$ were generated as prototypes for different activity patterns: pro $_{\mathrm{B}}$ was a symmetric burst model, and the other two were asymmetric ( pro $_{\mathrm{A}}$ was a "left-handed" burst model and pro $_{\mathrm{C}}$ was a "right-handed" burst model; see Figure 2 in [16]). The asymmetric models are similar to the two firing patterns shown in Figure 2. Physiologically, pro $_{\mathrm{A}}, \mathrm{pro}_{\mathrm{B}}$, and $\mathrm{pro}_{\mathrm{C}}$ presumably corresponded to decrementing, spindle-shaped, and incrementing EMG bursts, respectively. In the EMG of the rro $_{\mathrm{A}}$ model, the peak activity was at approximately the center of its active duration, whereas the peak activities of the asymmetric models were at the early and late stages of their active durations. The locations of the peak points on the time scale are definitely different among the three models. This difference suggests three different activity patterns of EMGs: The peak of the integrated EMG is located at an earlier point (close to zero on the horizontal axis) in the model $\operatorname{pro}_{\mathrm{B}}$ (see left column in Figure 2 in [16]), whereas it is located at later point in the $\mathrm{pro}_{\mathrm{C}}$ model (see right column in Figure 2 in [16]). The locations of the peak points differ, but the peak amplitudes, as well as the active durations, were not different among the three models.

\section{Application samples of the $T_{P}$ method and its derivatives}

In this subsection, several application samples of the $\mathrm{T}_{\mathrm{P}}$ method and its derivatives are introduced. Table 1 summarizes the major findings that are presented.

\subsection{Effects of food taste on suprahyoid activity patterns during swallowing}

\subsubsection{Five basic taste qualities}

Ten healthy young volunteers (five males and five females) were recruited as participants for the experiment. Each participant was recorded a SH surface EMG during swallowing of sample foods. A starch-based thickening agent, Mousse-up (MU, Nisshin Science, Yokohama, Japan), developed especially for dysphagic patients, was used as a basic material for the sample food. In this study, MU was dissolved in distilled water with taste substances for the five basic qualities (sweet, salty, sour, bitter, and umami); the tasteless sample food was prepared by dissolving MU in distilled water only. The used five taste substances and their concentrations were as follows: $0.5 \mathrm{M}$ of sucrose (SUC) for sweet tastes, $0.5 \mathrm{M}$ of sodium chloride $(\mathrm{NaCl})$ for salty tastes, $0.1 \mathrm{M}$ of hydrochloric acid $(\mathrm{HCl})$ for sour tastes, $0.0001 \mathrm{M}$ of quinine hydrochloride $(\mathrm{QHCl})$ for bitter tastes, and $0.02 \mathrm{M}$ of 
Quantitative Analysis of Activity Patterns in the Muscles of Mastication and Deglutition DOI: $h$ ttp://dx.doi.org/10.5772/intechopen.88108

\begin{tabular}{|c|c|c|c|c|c|c|}
\hline \multirow[t]{3}{*}{ Target } & \multirow[t]{3}{*}{ Parameter } & \multicolumn{3}{|c|}{ Food factors } & \multicolumn{2}{|c|}{ Human factors } \\
\hline & & \multicolumn{2}{|c|}{ Taste (chemical stimuli) } & \multirow{2}{*}{$\begin{array}{l}\text { Texture } \\
\text { and shape } \\
\text { (physical } \\
\text { stimuli) }\end{array}$} & \multirow[t]{2}{*}{ Aging } & \multirow{2}{*}{$\begin{array}{l}\text { Body } \\
\text { position }\end{array}$} \\
\hline & & $\begin{array}{l}\text { Five basic } \\
\text { taste } \\
\text { qualities }\end{array}$ & Umami & & & \\
\hline \multirow[t]{2}{*}{$\begin{array}{l}\text { Suprahyoid } \\
\text { (SH) } \\
\text { activity } \\
\text { pattern } \\
\text { during } \\
\text { swallowing }\end{array}$} & $\mathrm{T}_{\mathrm{P}}$ value & $\begin{array}{l}\text { Sourness } \\
\text { and } \\
\text { bitterness } \\
\text { in sample } \\
\text { foods } \\
\text { affected } \\
\text { (see 3.1.1) }\end{array}$ & $\begin{array}{l}\text { Higher } \\
\text { concentrations } \\
\text { of umami } \\
\text { substances } \\
\text { weakly } \\
\text { affected (see } \\
3.1 .2 \text { ) }\end{array}$ & $\begin{array}{l}\text { Textural } \\
\text { properties } \\
\text { of sample } \\
\text { foods } \\
\text { partly } \\
\text { affected } \\
\text { (see 3.2.2) }\end{array}$ & - & $\begin{array}{l}\text { Body } \\
\text { position } \\
\text { did not } \\
\text { affect } \\
\text { but did } \\
\text { affect } \\
\text { tongue } \\
\text { activity } \\
\text { pattern } \\
\text { (see 3.3) }\end{array}$ \\
\hline & $\mathrm{In}_{\mathrm{P}}$ value & - & $\begin{array}{l}\text { Higher } \\
\text { concentrations } \\
\text { of umami } \\
\text { substances } \\
\text { weakly } \\
\text { affected (see } \\
3.1 .2 \text { ) }\end{array}$ & - & $\begin{array}{l}\text { Aged } \\
\text { group } \\
\text { differed } \\
\text { from } \\
\text { young } \\
\text { and } \\
\text { middle- } \\
\text { aged } \\
\text { groups } \\
\text { (see } \\
\text { 3.2.1) }\end{array}$ & - \\
\hline $\begin{array}{l}\text { Masseteric } \\
\text { (Mass) } \\
\text { activity } \\
\text { pattern } \\
\text { during } \\
\text { chewing }\end{array}$ & $\mathrm{T}_{\mathrm{P}}$ value & $\begin{array}{l}\text { Sweetness } \\
\text { in sample } \\
\text { foods } \\
\text { affected } \\
\text { (see 3.4) }\end{array}$ & - & $\begin{array}{l}\text { Advance } \\
\text { of chewing } \\
\text { sequences } \\
\text { affected } \\
\text { (see 3.5.1 } \\
\text { and 3.5.2) }\end{array}$ & - & - \\
\hline
\end{tabular}

Numbers in parentheses indicate subsection numbers of '3. Application samples of the $T_{P}$ methods and its derivatives. $I n_{P}$ values are calculated by subtracting the preceding $T_{P-10}$ values (in 10 equally divided sections) from $T_{P}$ (see the text for details).

Table 1.

Summary of the major findings in this chapter.

monosodium glutamate (MSG) for umami tastes. Each participant was seated on a chair comfortably; approximately $8 \mathrm{~g}$ of one of the six sample foods (five with tastes and one without taste) at room temperature was placed randomly on the tongue (approximately $25^{\circ} \mathrm{C}$ ), and participants were instructed to swallow the sample in one gulp. Each trial was followed by a rinse of the mouth with a sufficient amount of tap water. Occurrence of swallowing was monitored with a concomitant record of laryngeal movement. Two sessions were repeated for each participant, and the two sessions were separated by at least 1 week.

The six averages of calculated $\mathrm{T}_{50}$ values ranged from $0.498 \pm 0.047$ (mean \pm standard deviation; for a sour sample food) to $0.572 \pm 0.070$ (for a tasteless sample food) in the first session and from $0.540 \pm 0.082$ (for a bitter sample food) to $0.571 \pm 0.073$ (for a sweet sample food) in the second session. The average $T_{50}$ value for the sour sample food was close to 0.500 , which was expected for half of the final cumulatively integrated SH EMG on the standardized time scale. In contrast, the average $T_{50}$ value in the tasteless sample food clearly exceeded 0.500 . The average $\mathrm{T}_{50}$ values imply that the $\mathrm{SH}$ muscles produced a spindle-shaped (or symmetric) firing pattern with the sour food and an incrementing firing pattern to the tasteless one (see "Necessity of a new parameter for 
activity pattern" section, especially Figure 1, and see Figure 2 in [16]). In addition to the $\mathrm{T}_{50}$ values, the subjective difficulty during swallowing was examined with a psychometric method used in a previous study [17]. The examination revealed that the sour and bitter sample foods increased the subjective difficulty of swallowing. The SH activity patterns evoked by the sour and bitter samples are congruent with the previous finding of subjective difficulty associated with swallowing of those foods [17].

Gustatory signals from foods were reported in humans and rats to provoke somatic responses in the trigeminal [18-20], facial [21], and hypoglossal nerves [22-25]. Receptors of four pairs of gustatory nerves can be responsible for gustation in the oral cavity, pharynx, and possibly larynx: the chorda tympani, glossopharyngeal, superior laryngeal nerves (see "Neural basis of mastication and deglutition" section), and, on the soft palate, the superficial petrosal nerve. In this study, the ingested foods probably stimulated the mucosae of the areas innervated by these cranial nerves during swallowing; consequently, gustatory signals arising from all of these nerves were likely to be involved in the findings. Gustatory signals from the glossopharyngeal and superior laryngeal nerve affect swallowing, especially the threshold for elicitation of swallowing [26-28]. Although animal experiments have revealed peripheral and central sensory mechanisms of the glossopharyngeal and superior laryngeal nerves (e.g., [29-32]), no studies have established whether gustatory signals from the two nerves contribute to sensory evaluation. In a previous study, the effect of taste and palatability of solutions in two (moderate and high) concentrations on peak lingual swallowing pressures was examined in 10 healthy adult participants [33]. Moderate SUC, high $\mathrm{NaCl}$, and high citric acid levels elicited higher peak lingual swallowing pressures than did water, whereas palatability did not. The previous results and the findings of this study may extend the knowledge about the effects of taste on swallowing movement.

\subsubsection{Umami substances}

Eight healthy young volunteers (three males and five females) were recruited as participants for this study. Sample foods consisted of MU dissolved in a mixture of MSG and IMP (disodium inosine-5'-monophosphate), in MSG alone, and in IMP alone. The procedures were identical to those in the first study (see "Five basic taste qualities" section for details). Analysis of $\mathrm{T}_{\mathrm{P}}$ values revealed no synergistic effects on SH EMG activity patterns during pharyngeal swallowing of umami foods. The $\mathrm{SH}$ activity patterns were consistent with those in the sensory evaluation of the subjective difficulty of swallowing, which was examined in the same participants. The consistency between SH EMG activity patterns and sensory evaluations of swallowing implies that both methods of data analyses did not discriminate between unitary umami foods (MU dissolved in MSG only or IMP only) and binary umami foods (MU dissolved in both MSG and IMP) or between non-umami foods (MU dissolved in SUC and in $\mathrm{NaCl}$ ) and tasteless ( $\mathrm{MU}$ dissolved in distilled water) foods. In view of the findings of a previous study [34], I can conclude that the $T_{P}$ method differentiates SH EMG activity patterns measured during pharyngeal swallowing of sour and bitter foods from those measured during swallowing of a tasteless food, although the method does not differentiate the activity patterns involved with sweet, salty, and umami foods (in both unitary and binary mixing solutions).

Both $\mathrm{T}_{\mathrm{P}}$ and $\mathrm{In}_{\mathrm{P}}$ values were calculated to analyze activity patterns of the $\mathrm{SH}$ EMGs during pharyngeal swallowing of samples with MU dissolved in unitary (either MSG or IMP) and binary (both MSG and IMP) umami substances at low and high concentrations as well as sweet and salty sample foods. A newly developed parameter, the $\operatorname{In}_{\mathrm{P}}$ value, was calculated by subtracting the preceding $\mathrm{T}_{\mathrm{P}-10}$ values (in 10 equally divided sections) from $\mathrm{T}_{\mathrm{P}}$ to improve precision in detecting 
differences. SH EMG activity patterns measured during pharyngeal swallowing differed between the low- and high-concentration umami samples, both unitary (IMP alone) and binary (both MSG and IMP) mixtures. According to the definition of the $\mathrm{T}_{\mathrm{P}}$ method, smaller $\mathrm{T}_{\mathrm{P}}$ values on a standardized time scale imply early cumulative $\mathrm{SH}$ activity corresponding to the percentile points. Six deciles of $\mathrm{T}_{\mathrm{P}}$ values for low-concentration umami sample foods (four $T_{P}$ values from $T_{50}$ to $\mathrm{T}_{80}$ in IMP sample foods and $\mathrm{T}_{10}$ and $\mathrm{T}_{30}$ in binary umami sample foods) were significantly smaller than those for higher concentration foods, which suggests that increased solvent concentrations affected activity patterns of the $\mathrm{SH}$ EMG during pharyngeal swallowing of the sample foods. Analysis of $T_{P}$ and $\operatorname{In}_{P}$ values showed that $\mathrm{SH}$ activity patterns differed slightly between low and higher concentrations of unitary and binary umami sample foods (see Figure 3 in [35]) but those $\mathrm{SH}$ activity patterns measured during swallowing of umami sample foods did not differ from those measured during swallowing of sweet, salty, or tasteless sample foods.

Synergism is an important concept that is widely recognized in the psychology and physiology of taste and one of the characteristic functional features of umami substances. In synergism, mixing two or more different tastes greatly enhances the umami taste. Such combinations include MSG, IMP, and disodium 5' -guanylate $[36,37]$. Gustatory signals evoked by ingested umami substances can modify not only feeding behaviors [38-40] but also visceral functions [41, 42]. This study is probably the first report to document changes in muscle activity patterns elicited by increasing concentrations of umami substances in foods. Although researchers previously reported changes in activity patterns of masticatory muscles during chewing sequences $[18,43,44]$, they examined conventional parameters, such as the number of chewing cycles, amplitudes, and durations of muscle activity to estimate activity patterns. Therefore, in these previous studies, activity patterns were evaluated indirectly by measurement of changes in conventional parameters; in contrast, my colleagues and I directly examined activity patterns of the SH during pharyngeal swallowing by using the $T_{P}$ method. In this study, I analyzed increments of $T_{P}$ values (i.e., $\operatorname{In}_{\mathrm{P}}$ values) first, and some significant differences were found in umami sample foods. Significant differences in both $\operatorname{In}_{P}$ values and $T_{P}$ values were detected only between low-concentration and high-concentration umami foods. This result suggests differential influences of umami taste in foods on activity patterns of the $\mathrm{SH}$ muscles during pharyngeal swallowing.

\subsection{Effects of food texture on suprahyoid activity patterns during swallowing}

\subsubsection{Aging of participants}

A total of 15 participants of both sexes were included in three participant groups: young adult (20 to 30 years old), middle-aged (40 to 50 years old), and elderly (60 to 70 years old). In each participant in the three groups, SH surface EMGs were recorded during swallowing of ordinary agar $(\mathrm{OA})$ and gelatin samples that had textural properties. Both $T_{P}$ and $\operatorname{In}_{P}$ values were calculated from the SH EMGs during swallowing. Statistical examination showed no significant differences in the average $T_{P}$ values among the three groups. In contrast, $\operatorname{In}_{P}$ values-derived from $\mathrm{T}_{\mathrm{P}}$ values_-in the elderly group differed in part from those of the other two groups for gelatin. This result suggests that (1) the overall activity pattern of the SH muscles is basically preserved in elderly people, but slight, partial changes occur with age, and (2) the $T_{P}$ method ( $\operatorname{In}_{\mathrm{P}}$ values) is useful in detecting the differences, although the differences with age were slight and partial. It is necessary to carefully analyze the characteristics of chewing and 
swallowing functions of elderly people to respond to the demand for production of special foods adjusted to the needs of some elderly persons in an aging society. In this study, $\mathrm{SH}$ activity patterns in three groups of differing ages were compared to evaluate whether aging affects activity patterns of muscles related to swallowing.

The analysis of data with $\operatorname{In}_{\mathrm{P}}$ values demonstrated a difference in SH activity patterns among the three groups during pharyngeal swallowing of gelatin. Rank correlation analyses revealed that the sequence of average $\operatorname{In}_{P}$ values in the elderly group differed from the sequences in the young adult and middle-aged groups for gelatin but not for OA. These results suggest that the difference in $\operatorname{In}_{\mathrm{P}}$ values among the three groups (see Figure 3 in [45]) is due to interactions between the properties of gelatin and the ages of participants. As an earlier report showed [46], textural properties of gelatin and OA clearly differ, and the properties of gelatin may cause the difference observed in $\operatorname{In}_{P}$ values. Because $T_{P}$ and $\operatorname{In}_{P}$ values are by nature not influenced by either amplitude or duration of EMG bursts [34, 35, 47], the differences in peak amplitude and active duration of the SH EMG had no bearing on the difference found in $\operatorname{In}_{\mathrm{P}}$ values. The freedom from the amplitude and active duration should be an advantage of the $T_{P}$ method, and it allows the comparison of activity patterns in EMGs recorded in different trials and participants.

\subsubsection{Food texture properties}

Five healthy young volunteers (two males and three females) were recruited as participants for this study. Three sample foods with MU in low concentrations $(3.0 \%$; 3.0MU), medium concentrations $(6.0 \%$; 6.0MU), and high concentrations $(9.0 \% ; 9.0 \mathrm{MU})$ at room temperature (approximately $25^{\circ} \mathrm{C}$ ) were used. Before these food samples were given to participants, three textural properties (hardness, adhesiveness, and cohesiveness) were measured by a texturometer. One of the sample foods was selected randomly and delivered to each participant. EMG electrodes were attached to the participant for SH activity, and the participant was seated on a chair and instructed to accept the sample food. Three $T_{P}$ values $\left(T_{20}, T_{50}\right.$, and $\left.T_{80}\right)$ were calculated and analyzed in this study. The average $\mathrm{T}_{50}$ value for $6.0 \mathrm{MU}$ was significantly larger than that for 3.0MU but not that for 9.0MU, whereas there was no difference in average $\mathrm{T}_{20}$ and $\mathrm{T}_{80}$ values between the three sample foods. The averages of $\mathrm{SH}$ durations and cumulative $\mathrm{SH}$ activity and the ratios between these two parameters were measured as well. The average SH duration for 9.0MU was longer than that for 3.0MU, but there were no significant differences in the cumulative SH EMG activity or in the ratios of the used sample foods.

The hardness, adhesiveness, and cohesiveness values of the used three MU sample foods on average increased as the concentration of MU increased (see Figure 2 in [48]). The hardness of 6.0MU and 9.0MU was much larger than that of $3.0 \mathrm{MU}$ (about $350 \%$ and $450 \%$, respectively). Similar large differences between 3.0MU and the other two were shown in the adhesiveness and cohesiveness as well, and statistical examinations demonstrated significant differences in these three textural parameters among the three samples of MU. Previous studies documented that increasing the hardness, viscosity, and volume of sample foods prolongs the durations of oral and pharyngeal swallowing (e.g., $[49,50]$ ). Similarly, a videofluoroscopic study also documented that swallowing highdensity barium, in comparison with low-density barium, prolongs the oral and pharyngeal transit times in healthy participants [49]. Moreover, an electromyographic and manometric study demonstrated that the average duration of the $\mathrm{SH}$ activity during the swallowing of high-density agar fluid was longer than that of low-density agar $[34,35,47]$. Consequently, I can conclude that increasing the 
textural properties of the sample foods is responsible for prolonging the duration of pharyngeal swallowing.

\subsection{Effects of body position on suprahyoid activity patterns during swallowing}

Nine healthy young volunteers (six males and three females) participated in this study, and surface EMGs were recorded from the anterior tongue and $\mathrm{SH}$ muscles of each participant. Three sample foods with different concentrations (2.0MU, 5.7MU, and 9.1MU) of $\mathrm{MU}$ at the room temperature (approximately $25^{\circ} \mathrm{C}$ ) were used.

Each participant was positioned randomly for swallowing at one of the four angles: horizontal supine (0 degrees), 30 degrees inclined, 60 degrees inclined, and upright (90 degrees). The food sample was selected randomly among from the foods with three MU concentrations. Analysis of $\mathrm{T}_{50}$ values showed that the average $\mathrm{T}_{50}$ value of the activity patterns in the anterior tongue in the horizontal supine position was significantly higher than that in the upright position, but there were no significant differences in anterior tongue $\mathrm{T}_{50}$ values between the foods with the three concentrations. In contrast, analysis of $\mathrm{SH} \mathrm{T}_{50}$ values showed that the average $\mathrm{T}_{50}$ value for 9.1MU was significantly higher than that of $2.0 \mathrm{MU}$, but there were no significant differences in $\mathrm{SH} \mathrm{T}_{50}$ values between the four body positions. The results indicated that anterior tongue activity pattern was altered from a decrementing firing pattern to an incrementing pattern by the shift from the upright position to the horizontal supine position, whereas $\mathrm{SH}$ activity pattern was altered from a decrementing firing pattern to an incrementing pattern by the shift from the low-concentration food to a high-concentration one.

According to the findings in this study, activity patterns of the anterior tongue and $\mathrm{SH}$ muscles are affected by both body positions and food properties. Figure 4 demonstrates $\mathrm{SH}$ activities during swallowing of two concentrations, 2.0MU and 9.1MU, at four body positions, horizontal supine (0 degrees), 30 degrees inclined, 60 degrees inclined, and upright (90 degrees), in a healthy young male participant. Visual observation of this figure suggests that activity patterns seem similar among the four body positions but different between the two sample foods. Actually, four $\mathrm{T}_{50}$ values calculated for the 2.0MU are 0.414 (for 0 degrees), 0.450 (for 30 degrees), 0.522 (60 degrees), and 0.462 (90 degrees), whereas those for the 9.1MU are 0.625 (for 0 degrees), 0.509 (for 30 degrees), 0.622 (60 degrees), and 0.618 (90 degrees). The averaged $\mathrm{T}_{50}$ value for the 2.0MU (0.508) tended to be smaller than that for the 9.1MU (0.607). Because in general a smaller $\mathrm{T}_{\mathrm{P}}$ value means relatively shorter time for the half of the integrated EMG and corresponds to a more active burst, the SH muscles demonstrated more activity in the first half of swallowing of the 2.0MU than that of the 9.1MU. Thus the $\mathrm{T}_{50}$ values calculated indicate that the two concentrations of the sample food affect activity patterns of the $\mathrm{SH}$ muscles during swallowing, but body positions do not. Precise analysis of the effects of body positions and thickener's concentrations is discussed later, but swallowing movement is not constant among body positions. Besides, activity patterns of the tongue were analyzed in the article described next because it is widely accepted that the tongue, as well as the SH muscles, plays an important role in swallowing (e.g., [5]).

According to the nature of the $\mathrm{T}_{\mathrm{P}}$ values [16], the obtained results physiologically implied that the anterior tongue EMG during swallowing expressed a decrementing firing pattern in the upright position (with smaller $\mathrm{T}_{50}$ values) and an incrementing firing pattern in the horizontal supine position (with larger $\mathrm{T}_{50}$ values). Previous papers [51-53] documented that the four body positions affected neither durational nor amplitude parameters of the anterior tongue EMG during swallowing; the body positions modified only the activity patterns of the anterior tongue. The tongue contains skeletal muscles without attachment to the bone, 
intrinsic muscles (longitudinal, transverse, and vertical muscle groups), other muscles attached to at least one bone, and extrinsic muscles (genioglossus, hyoglossus, and styloglossus) [54]. The electrodes were attached to the surface of the anterior tongue in this study [55], and so the EMG recording might have originated from the intrinsic muscles of the tongue, but it is very difficult to determine the origin precisely by the use of surface electrodes.

\subsection{Effects of food taste on masseteric activity patterns during chewing}

Ten healthy young volunteers (seven males and three females) were recruited as participants in this study. A surface EMG was recorded from the $\mathrm{SH}$ of each participant, and three $\mathrm{T}_{\mathrm{P}}\left(\mathrm{T}_{25}, \mathrm{~T}_{50}\right.$, and $\left.\mathrm{T}_{75}\right)$ values were calculated from the EMG data $\left(200 \mathrm{~T}_{\mathrm{P}}\right.$ values for each GC). Four fruit GCs (apple-, grape-, orange-, and pearflavored) were used as sample foods. Four textural properties (hardness, adhesiveness, cohesiveness, and gumminess) and chemical components (sugars and organic acids) of the GCs were analyzed. The hierarchical cluster analysis was applied to the three $T_{P}$ values collected with each GC. The standardized Euclidean distance and the Ward linkage method were used for cluster amalgamation. Two predominant sugars, SUC and maltose, and four organic acids (citric, malic, phosphoric, and tartaric acids) were detected by the chemical analysis. In the cluster analysis, the $\mathrm{T}_{25}$ and $T_{75}$ values were classified into four subclusters, and the $T_{50}$ values were classified into three subclusters. In two $\mathrm{T}_{75}$ subclusters, the combined amounts of the two predominant sugars (SUC and maltose) differed significantly, but neither the four detected organic acids nor the textural properties differed. These findings indicate that SUC and maltose in GCs can affect masseteric activity patterns during chewing, particularly in the later stages, but their organic acids and textural properties do not have such an effect.

The authors of this study used a hierarchical cluster analysis method, followed by an ANOVA, to evaluate the textural properties and chemical components of individual sample foods corresponding to the clustered data for the three $\mathrm{T}_{\mathrm{P}}$ values. The reason for the use of the analyses was that the same sample food item (1) could not be consumed by the participants, (2) could not be used for the textural measurements, and (3) could not be used for chemical analyses. Therefore, it was necessary to collect the textural properties and chemical components of each sample food separately, calculate their averages individually, and examine the correlations between $\mathrm{T}_{\mathrm{P}}$ values and these food properties. In the hierarchical cluster analysis with the standardized Euclidean distance and Ward linkage methods, $T_{P}$ values were fragmented according to their similarity and then grouped into three and four subclusters when the amalgamation level was established at $12 \%$ of the greatest distance in each dendrogram of $\mathrm{T}_{25}, \mathrm{~T}_{50}$, and $\mathrm{T}_{75}$ values. ANOVA could be used to compare the subclusters in terms of their textural properties and chemical compositions because each $\mathrm{T}_{\mathrm{P}}$ value in the subclusters was associated with the specific textural properties and chemical compositions of each sample food.

In previous physiological studies in humans $[18,19,38,56,57]$ and rats [20], investigators analyzed masseteric activity during chewing of various foods, as well as ingestion of liquids, with different tastes and textures. Activity patterns were evaluated indirectly by visual observation or with the use of parameters such as the number of chews, chewing time, and mean voltage of the masseteric activity. In none of these studies did the investigators use specific parameters for the evaluations. The use of specific parameters, such as $T_{P}$ values, is essential for the precise evaluation of muscle activity patterns. In this study, the average $\mathrm{T}_{75}$ value measured during the chewing of the orange-flavored GC $(0.725 \pm 0.008)$ was lower than that measured during the chewing of the apple-flavored GC $(0.767 \pm 0.008)$. 
As mentioned in other application samples, these $T_{75}$ values imply that chewing the orange-flavored GC evoked a decrementing firing pattern in the Mass, which is characterized by smaller $\mathrm{T}_{\mathrm{P}}$ values, and chewing the apple-flavored GC evoked an incrementing firing pattern, although the difference in the firing patterns is just "relative," especially in such a small difference in $T_{P}$ values $(0.725$ versus 0.767$)$.

\subsection{Effects of food texture on masseteric activity patterns during chewing}

\subsubsection{Food texture properties}

Eight healthy young volunteers (all males) were recruited as participants in this study. A surface EMG was recorded from the Mass of each participant, and nine $T_{P}$ values (from $T_{10}$ to $T_{90}$ ) were calculated from the EMG data. The two agars, OA and Ina-agar (IA), each at two concentrations, were used as sample foods: $0.5 \%$ OA (0.5OA), $1.5 \%$ OA (1.5OA), $2.5 \%$ IA (2.5IA), and $4.0 \%$ IA (4.0IA). The four sample foods differed partly in four textural properties: hardness, fracturability (defined as the force of the significant break in the curve on the first compression [bite]), adhesiveness, and cohesiveness. For example, the highest average hardness, $4.05 \pm 0.08\left(\times 10^{4} \mathrm{~Pa}\right)$, and the highest average adhesiveness, $65.3 \pm 7.2\left(\mathrm{~J} / \mathrm{m}^{3}\right)$, were observed in the $1.5 \mathrm{OA}$, whereas the lowest average values, $0.94 \pm 0.04\left(\times 10^{4} \mathrm{~Pa}\right)$ and $2.9 \pm 1.1\left(\mathrm{~J} / \mathrm{m}^{3}\right)$, were observed in 2.5IA. One of the four sample foods, selected randomly, was delivered to each participant. In addition to the $\mathrm{T}_{\mathrm{P}}$ values, other four parameters (mastication time, number of chewing, cumulative masseteric activity, and amplitude rate) were analyzed. The average $T_{50}$ values in the first cycles, for example, showed the lowest value for 2.5IA (0.515 \pm 0.021$)$ and highest value for $1.5 \mathrm{OA}(0.535 \pm 0.025)$. Statistical analysis with Wilcoxon's test revealed significant differences in $T_{P}$ values between the first and last cycles with 1.5OA (four of the nine $\mathrm{T}_{\mathrm{P}}$ deciles) and with 2.5IA (five). The average $T_{P}$ values of the first cycle tended to be larger, but not significantly so, than those of the last cycle (see Figure 3 in [47]).

One of the major findings of this study was the significant differences in $T_{P}$ values detected between the first and last cycles. The finding suggests that sequential changes in masseteric activity patterns occur at the beginning and end of chewing. In previous studies, researchers reported changes in activity patterns of masticatory muscles during chewing sequences by measuring conventional parameters, such as the number of chewing cycles, and amplitude and duration of muscle activity (e.g., $[18,43,44])$. This study, in which the $T_{P}$ method was used, added new information about sequential changes in activity patterns of the Mass. The result indicates that the masseteric activity observed in chewing 2.5IA reached the half of the final cumulative masseteric activity slightly earlier than it did with the other three agars. However, the average $T_{P}$ values between the used four agars were not significantly different between the first chewing cycles or between last chewing cycles. The result suggests that hardness and other textural properties measured before chewing do not affect the masseteric activity patterns, at least in the first and last cycles of the chewing sequences.

\subsubsection{Food shapes and textures}

Ten healthy young volunteers (seven males and three females) were recruited as participants in this study. A surface EMG was recorded from the Mass of each participant, and three $\mathrm{T}_{\mathrm{P}}$ values $\left(\mathrm{T}_{25}, \mathrm{~T}_{50}\right.$, and $\left.\mathrm{T}_{75}\right)$ were calculated from the EMG data. Six foods (cheese, GC, marshmallow, prune, rice cracker, and sponge cake) were used as samples. These sample foods differed partly in three shape dimensions 
(length, width, and height) and in four textural properties (hardness, fracturability, adhesiveness, and cohesiveness). One of the six sample foods, selected randomly, was delivered to each participant. In addition to the three $T_{P}$ values, other three parameters (number of chewing, sequence length of chewing, and active duration of masseteric activity) were analyzed. The major results by linear model analysis in relation to textural properties of the sample foods showed that (1) the $\mathrm{T}_{25}, \mathrm{~T}_{50}$, and $\mathrm{T}_{75}$ values were increased by $0.015,0.020$, and 0.021 points, respectively, by a $100-\mathrm{kPa}$ increase in the hardness; $(2)$ the $\mathrm{T}_{25}$ and $\mathrm{T}_{50}$ values were decreased by 0.061 and 0.070 points, respectively, by a $100-\mathrm{kPa}$ increase in the fracturability; and (3) the number of chewing cycles was reduced by 8.8 cycles by a $100-\mathrm{kPa}$ increase in the fracturability and 6.6 cycles by a $5.0-\mathrm{kJ} / \mathrm{m}^{3}$ increase in the adhesiveness. These findings suggest that the combination of several parameters can enhance discriminability between foods owing to differential sensitivity to food properties.

The $\mathrm{T}_{\mathrm{P}}$ method was used to evaluate the masseteric activity patterns during the chewing of the six sample foods with different textural properties. Cheese differed from GC, marshmallow, rice cracker, and sponge cake in all three $T_{P}$ values $\left(T_{25}, T_{50}\right.$, and $\mathrm{T}_{75}$; see Figure 4 in [58]). The differences between $\mathrm{T}_{\mathrm{P}}$ values raise a question: How can these results best be interpreted? One possible interpretation may be that there was relatively more activity during the early period of the masseteric EMG during the chewing of cheese than there was during the chewing of GC. The difference resulted in different cumulative masseteric EMG curves: The masseteric EMG curve for cheese exceeded the line that indicated theoretically tonic activity of the EMG throughout

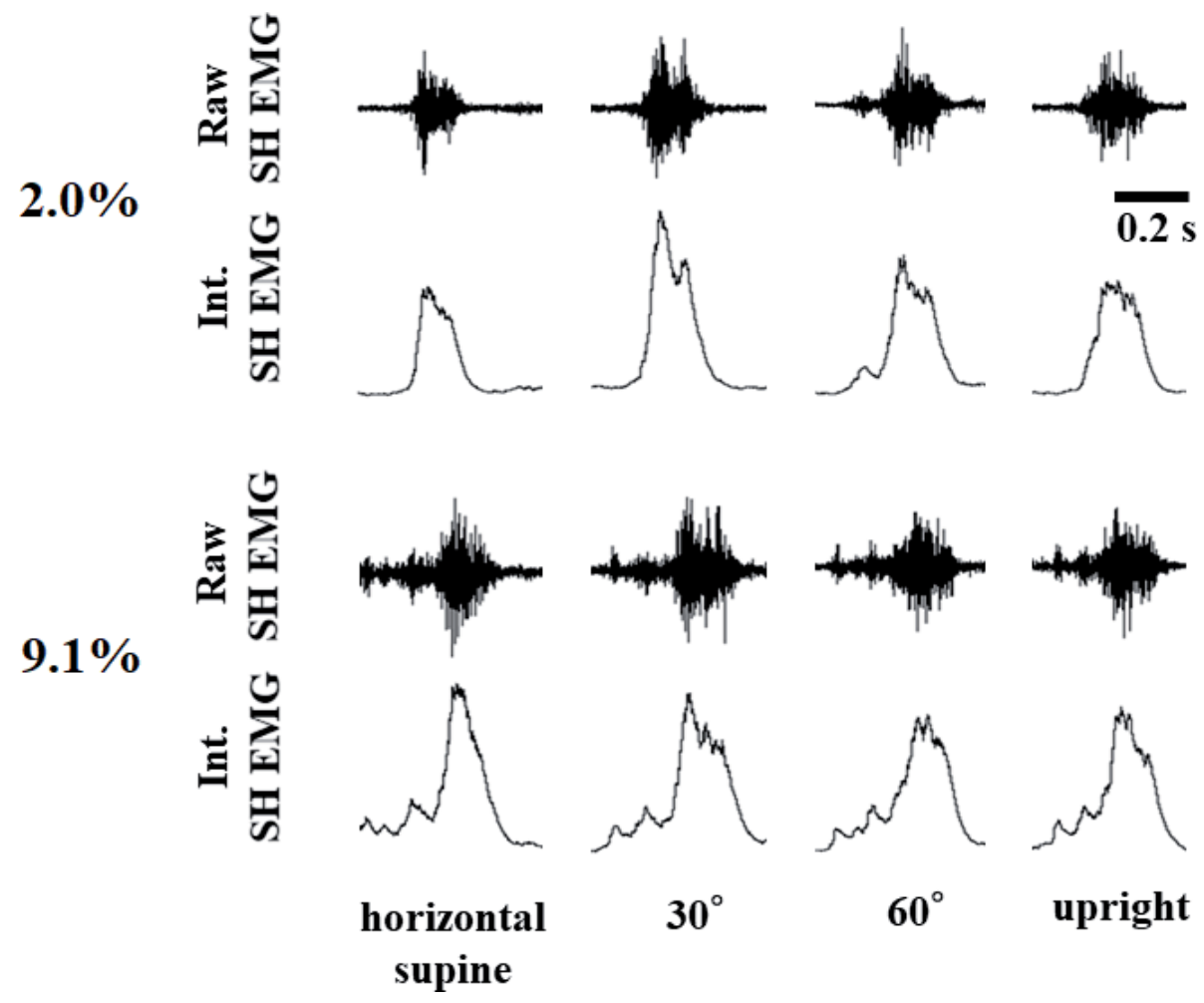

Figure 4.

Comparison of SH EMG among four body positions and between two concentrations of sample foods. The SH EMG and integrated SH EMG were recorded during swallowing of two sample foods with low (2.0\%) and high (9.1\%) concentrations of a thickening agent in four body positions: horizontal supine, 30 degrees inclined, 60 degrees inclined, and upright. Scales for the horizontal and vertical axes are omitted for clarity. Raw SH EMG: raw suprahyoid electromyogram; Int. SH EMG: integrated suprahyoid electromyogram. 
its active duration, whereas the masseteric EMG curve for GC fell below this line. The interpretation suggests that the masseteric EMG of cheese shows a decrementing firing pattern, which is characterized by smaller $T_{P}$ values, during the first chewing cycle in comparison with the masseteric EMGs of the GC, marshmallow, rice cracker, and sponge cake. However, it should be noted that there are no absolute criteria that allow an investigator to discriminate between incrementing and decrementing firing patterns of EMG activity. In other words, it is not possible to say that an EMG activity pattern is a decrementing one if the $\mathrm{T}_{25}$ value exceeds 0.300 points.

The regression coefficients estimated in the linear model analysis for the three $\mathrm{T}_{\mathrm{P}}$ values were not particularly large with regard to the hardness of the sample food used. These regression coefficients were all statistically significant, but the result suggests that hardness only weakly contributes to the masseteric activity patterns evaluated by $\mathrm{T}_{\mathrm{P}}$ values. Ten deciles (e.g., $\mathrm{T}_{10}$ and $\mathrm{T}_{20}$ values) were used in previous studies [34, 35, 53, 59], and the use of 10 deciles may provide more information about EMG activity patterns than quartiles $\left(T_{25}, T_{50}, T_{75}\right.$ values $)$. However, my colleagues and I found that it was often difficult to interpret the 10 decile results in this study. According to our experience of using both $\mathrm{T}_{\mathrm{P}}$ values in the previous and current studies, $\mathrm{T}_{50}$ values can be compared with each other. The $\mathrm{T}_{50}$ values differed slightly from those in the previous studies $[34,35,53,59]$, but statistical testing was not performed to determine the significance of these differences. It is difficult to know whether these differences reflect the difference in the muscles used ( $\mathrm{SH}$ versus Mass) or the difference in functions examined (swallowing versus chewing). Suitability of using $\mathrm{T}_{50}$ values or other $\mathrm{T}_{\mathrm{P}}$ values may be dependent on the functions.

\section{Advantages, limitations, and perspectives of the $T_{P}$ method}

The $T_{P}$ method can have at least three advantages. The first advantage is that the calculation from numerical data is simple and the digitization to numeral data from the original records is now common in most laboratories. The calculation itself is surely simple, but my colleagues and I needed to develop a special software for the calculation on a personal computer because of the huge amounts of data. Indeed, my colleagues and I had to handle 100,000 rows of a data sheet for numerical EMG data of only $10 \mathrm{~s}$ in the calculation of a $\mathrm{T}_{\mathrm{P}}$ value. The second advantage is the ease of comparison of EMG data with largely different amplitudes because of the nature of the $\mathrm{T}_{\mathrm{P}}$ method (see "Development and verification of a $\mathrm{T}_{\mathrm{P}}$ method" section): that is, the relative time corresponding to the final cumulated SH EMG is important, although the absolute amplitudes are not. The third advantage is the independence of analysis from active durations of the EMG in the $T_{P}$ method, as in the second advantage.

The validity of the $T_{P}$ method was examined mathematically and theoretically by a computer simulation (see "Development and verification of a $\mathrm{T}_{\mathrm{P}}$ method" section) but was not experimentally. This is the first and major limitation of the $T_{P}$ method. It seems to be effective for the verification experimentally by kinesiological analysis of chewing and swallowing movements with concomitant recording of muscle activities. The analysis can provide useful information about functional meanings of differences in $\mathrm{T}_{\mathrm{P}}$ values among recorded $\mathrm{EMG}$ bursts. The second limitation is based on the nature of the $T_{P}$ method: $T_{P}$ values are calculated from a cumulative EMG, and so the calculated values are not independent in each burst. It is meaningless to compare two or more $\mathrm{T}_{\mathrm{P}}$ values (e.g., $\mathrm{T}_{20}$ and $\mathrm{T}_{40}$ values) of an EMG burst. However, the problem seems not to be serious, inasmuch as such a comparison rarely occurs and the same $\mathrm{T}_{\mathrm{P}}$ values (e.g., $\mathrm{T}_{50}$ and $\mathrm{T}_{50}{ }_{50}$ ) in different EMG bursts are usually compared for quantitative evaluation of two or more activity patterns of the EMG. 
The $\mathrm{T}_{\mathrm{P}}$ method is just a tool for analyzing muscle activity patterns and can provide quantitative information for the patterns but not any direct cues for the central nervous system mechanism responsible for the patterns. Thus it is necessary to conduct experiments in which data of both central and peripheral nervous system muscle activities are recorded. Concomitant analysis of these activities can be used to explore the central nervous mechanism, and the $\mathrm{T}_{\mathrm{P}}$ method should be useful for analysis of peripheral muscle activity. As shown previously (see "Application samples of the $T_{P}$ method"), the $T_{P}$ method is useful for evaluating activity patterns of EMGs quantitatively. The method is applicable to various EMG bursts with different amplitudes and active durations observed during mastication and deglutition. This chapter focused on these two functions only, but the $\mathrm{T}_{\mathrm{P}}$ method must be also applicable to muscle activities of other movements, such as respiration and locomotion, because these movements have neural mechanisms similar or common to those of mastication and deglutition (e.g., [60-62]).

As summarized in Table 1, in this chapter I showed application samples that were related to chewing and swallowing functions in healthy participants. However, the $\mathrm{T}_{\mathrm{P}}$ method should be applicable to other cyclic functions, such as breathing, walking, and swimming. Besides, the $\mathrm{T}_{\mathrm{P}}$ method should also be applicable to wider research fields and clinical sites, especially as one of the diagnostic tools for patients with motor diseases. For example, disorders in the motor system may command abnormal signals to the target muscle(s), and activity patterns of the EMG may reflect the abnormal command signals.

\section{Conclusions}

Activity patterns of the Mass EMG during chewing and the SH EMG during swallowing were analyzed with a $\mathrm{T}_{\mathrm{P}}$ method. The $\mathrm{T}_{\mathrm{P}}$ method compared activity patterns of individual EMG bursts with different amplitudes and active durations on standardized time scales free from changes in the amplitudes and active durations. Five groups of application samples were introduced to compare the activity patterns of these EMGs during chewing and swallowing at different body positions of participants as well as by different tastes and textures of sample foods.

\section{Acknowledgements}

I deeply thank the following colleagues who kindly encourage me to continue the research: Drs. Ichiro Ashida, Hajime Iwamori, Takako Yamazaki, Naoko Ito, Shin-ya Kawakami (Department of Health and Nutrition, Niigata University of Health and Welfare), Satomi Miyaoka (Department of Mental Health Science, Graduate School of Rehabilitation, Niigata University of Rehabilitation), Yuko Tamaki (Department of Food Science, Otsuma Women's University), and Daigo Inagaki (Inagaki Dental Clinic). The studies shown in this chapter were supported in part by Grants-in-Aid for Scientific Research from the Ministry of Education, Science and Culture of Japan (No. 19500667 and No. 22500740). I would like to thank Enago (www.enago. jp) for the English language review.

\section{Conflict of interest}

The author reports no conflicts of interest. The author alone is responsible for the contents and writing of this book chapter. 


\section{Author details}

Yozo Miyaoka

Department of Health and Nutrition, Niigata University of Health and Welfare, Niigata, Japan

*Address all correspondence to: miyaoka@nuhw.ac.jp

\section{IntechOpen}

(C) 2019 The Author(s). Licensee IntechOpen. This chapter is distributed under the terms of the Creative Commons Attribution License (http://creativecommons.org/licenses/ by/3.0), which permits unrestricted use, distribution, and reproduction in any medium, provided the original work is properly cited. (cc) BY 


\section{References}

[1] Yamada Y, Yamamura K, Inoue $\mathrm{M}$. Coordination of cranial motoneurons during mastication. Respiratory Physiology \& Neurobiology. 2005;147(2-3):177-189. DOI: 10.1016/j. resp.2005.02.017

[2] Groher ME. Dysphagia : Diagnosis and Management. 3rd ed. Boston: Butterworth-Heinemann; 1997

[3] Sato K, Umeno H, Chitose S, Nakashima T. Deglutition and respiratory patterns during swallowing. The Japan Journal of Logopedics and Phoniatrics. 2011;52:132-140 (In Japanese with English abstract)

[4] Doty RW, Bosma JF. An electromyographic analysis of reflex deglutition. Journal of Neurophysiology. 1956;19(1):44-60. DOI: 10.1152/ jn.1956.19.1.44

[5] Miller AJ. The Neuroscientific Principles of Swallowing and Dysphagia. San Diego: Singular Pub. Group; 1999

[6] Logemann JA. Evaluation and Treatment of Swallowing Disorders. 2nd ed. Austin, Tex: PRO-ED; 1998

\section{[7] Ogawa H, Imoto T, Hayama T.}

Responsiveness of solitario-parabrachial relay neurons to taste and mechanical stimulation applied to the oral cavity in rats. Experimental Brain Research. 1984;54(2):349-358

[8] Ogawa H, Sato M, Yamashita S. Multiple sensitivity of chorda tympani fibres of the rat and hamster to gustatory and thermal stimuli. The Journal of Physiology. 1968;199(1):223-240

[9] Ogawa H, Hayama T, Ito S. Response properties of the parabrachio-thalamic taste and mechanoreceptive neurons in rats. Experimental Brain Research. 1987;68(3):449-457

[10] Nomura T, Ogawa H. The taste and mechanical response properties of neurons in the parvicellular part of the thalamic posteromedial ventral nucleus of the rat. Neuroscience Research. 1985;3(2):91-105

[11] Yamamoto T, Matsuo R, Kiyomitsu Y, Kitamura R. Sensory inputs from the oral region to the cerebral cortex in behaving rats: An analysis of unit responses in cortical somatosensory and taste areas during ingestive behavior. Journal of Neurophysiology. 1988;60 (4):1303-1321. DOI: 10.1152/ jn.1988.60.4.1303

[12] Brown WE, Shearn M, MacFie HJH. Method to investigate differences in chewing behaviour in humans: II. Use of electromyography during chewing to assess chewing behaviour. Journal of Texture Studies. 1994;25(1):17-31. DOI: 10.1111/j.1745-4603.1994.tb00752.x

[13] Horio T, Kawamura Y. Effects of texture of food on chewing patterns in the human subject. Journal of Oral Rehabilitation. 1989;16(2):177-183

[14] Mioche L, Bourdiol P, Martin JF, Noel Y. Variations in human masseter and temporalis muscle activity related to food texture during free and sideimposed mastication. Archives of Oral Biology. 1999;44(12):1005-1012

[15] Plesh O, Bishop B, McCall W. Effect of gum hardness on chewing pattern. Experimental Neurology. 1986;92(3):502-512

[16] Ashida I, Kawakami S, Miyaoka Y. A new method of simulating surface electromyograms using probability density functions. Computers in Biology and Medicine. 2008;38(7):837-844. DOI: 10.1016/j. compbiomed.2008.05.001 
[17] Miyaoka Y, Haishima K, Takagi M, Haishima H, Asari J, Yamada Y. Influences of thermal and gustatory characteristics on sensory and motor aspects of swallowing. Dysphagia. 2006;21(1):3848. DOI: $10.1007 / \mathrm{s} 00455-005-9003-6$

[18] Gonzalez R, Sifre S, Benedito J, Nogues V. Comparison of electromyographic pattern of sensory experts and untrained subjects during chewing of Mahon cheese. The Journal of Dairy Research. 2002;69(1):151-161

[19] Neyraud E, Peyron MA, Vieira C, Dransfield E. Influence of bitter taste on mastication pattern. Journal of Dental Research. 2005;84(3):250-254. DOI: $10.1177 / 154405910508400308$

[20] Yamamoto T, Matsuo R, Fujiwara T, Kawamura Y. EMG activities of masticatory muscles during licking in rats. Physiology \& Behavior. 1982;29(5):905-913

[21] Steiner JE. The gustofacial response: Observation on normal and anencephalic newborn infants. In: Bosma JF, editor. Symposium on Oral Sensation and Perception-IV. NIHDHEW; 1973. p. 254-278

[22] Dinardo LA, Travers JB. Hypoglossal neural activity during ingestion and rejection in the awake rat. Journal of Neurophysiology. 1994;72(3):1181-1191. DOI: 10.1152/jn.1994.72.3.1181

[23] Nowlis GH. Taste-elicited tongue movements in human newborn infants: An approach to palatability. Symposium on Oral Sensation and Perception. 1973; (4):292-310

[24] Yamamoto T. Linguo-hypoglossal reflex: Effects of mechanical, thermal and taste stimuli. Brain Research. 1975;92(3):499-504

[25] Yamamoto T, Fujiwara T, Matsuo R, Kawamura Y. Hypoglossal motor nerve activity elicited by taste and thermal stimuli applied to the tongue in rats. Brain Research. 1982;238(1):89-104

[26] Kajii Y, Shingai T, Kitagawa J, Takahashi Y, Taguchi Y, Noda T, et al. Sour taste stimulation facilitates reflex swallowing from the pharynx and larynx in the rat. Physiology \& Behavior. 2002;77(2-3):321-325

[27] Logemann JA, Pauloski BR, Colangelo L, Lazarus C, Fujiu M, Kahrilas PJ. Effects of a sour bolus on oropharyngeal swallowing measures in patients with neurogenic dysphagia. Journal of Speech and Hearing Research. 1995;38(3):556-563

[28] Shingai T, Miyaoka Y, Ikarashi R, Shimada K. Swallowing reflex elicited by water and taste solutions in humans. The American Journal of Physiology. 1989;256 (4 Pt 2):R822-R826. DOI: 10.1152/ajpregu.1989.256.4.R822.

[29] Dickman JD, Smith DV. Response properties of fibers in the hamster superior laryngeal nerve. Brain Research. 1988;450(1-2):25-38

[30] Frank ME. Taste-responsive neurons of the glossopharyngeal nerve of the rat. Journal of Neurophysiology. 1991;65(6):1452-1463. DOI: 10.1152/ jn.1991.65.6.1452

[31] Shingai T, Beidler LM. Response characteristics of three taste nerves in mice. Brain Research. 1985;335(2):245-249

[32] Smith DV, Hanamori T. Organization of gustatory sensitivities in hamster superior laryngeal nerve fibers. Journal of Neurophysiology. 1991;65(5):1098-1114. DOI: 10.1152/ jn.1991.65.5.1098

[33] Pelletier CA, Dhanaraj GE. The effect of taste and palatability on lingual swallowing pressure. Dysphagia. 2006;21(2):121-128. DOI: $10.1007 /$ s00455-006-9020-0 
[34] Miyaoka Y, Ashida I, Inagaki D, Kawakami S. Differentiation of activity patterns in the suprahyoid muscles during swallowing of foods with five taste qualities. Journal of Sensory Studies. 2005;20(6):473-483. DOI: 10.1111/j.1745-459X.2005.00041.x

[35] Miyaoka Y, Ashida I, Kawakami S, Miyaoka S. Differentiation of suprahyoid activity patterns during swallowing of umamitasting foods. Journal of Sensory Studies. 2006;21(6):572-583. DOI: 10.1111/j.1745-459X.2006.00083.x

[36] Yamaguchi S. The synergistic taste effect of monosodium glutamate and disodium 5'-inosinate. Journal of Food Science. 1967;32(4):473-478. DOI: 10.1111/j.1365-2621.1967.tb09715.x

[37] Rifkin B, Bartoshuk LM. Taste synergism between monosodium glutamate and disodium 5 '-guanylate. Physiology \& Behavior. 1980;24(6):1169-1172

[38] Horio T. EMG activities of facial and chewing muscles of human adults in response to taste stimuli. Perceptual and Motor Skills. 2003;97(1):289-298. DOI: 10.2466/pms.2003.97.1.289

[39] Prescott J. Effects of added glutamate on liking for novel food flavors. Appetite. 2004;42(2):143-150. DOI: 10.1016/j.appet.2003.08.013

[40] Viarouge C, Even P, Rougeot C, Nicolaidis S. Effects on metabolic and hormonal parameters of monosodium glutamate (umami taste) ingestion in the rat. Physiology \& Behavior. 1991;49(5):1013-1018

[41] Horio T. Effects of various taste stimuli on heart rate in humans. Chemical Senses. 2000;25(2):149-153

[42] Niijima A, Togiyama T, Adachi A. Cephalic-phase insulin release induced by taste stimulus of monosodium glutamate (umami taste). Physiology \& Behavior. 1990;48(6):905-908

[43] Kohyama K, Yamaguchi M, Kobori C, Nakayama Y, Hayakawa F, Sasaki T. Mastication effort estimated by electromyography for cooked rice of differing water content. Bioscience, Biotechnology, and Biochemistry. 2005;69(9):1669-1676. DOI: 10.1271/ bbb.69.1669

[44] Peyron MA, Lassauzay C, Woda A. Effects of increased hardness on jaw movement and muscle activity during chewing of visco-elastic model foods. Experimental Brain Research. 2002;142(1):41-51. DOI: 10.1007/ s00221-001-0916-5

[45] Miyaoka Y, Ashida I, Kawakami S, Miyaoka S, Igarashi A, Yamada Y. Aging-related influences on activity patterns in the suprahyoid muscles during swallowing: Preliminary analysis. Journal of Sensory Studies. 2007;22(4):394-402. DOI: 10.1111/j.1745-459X.2007.00113.x

[46] Igarashi A, Arai E, Watanabe R, Miyaoka Y, Tazawa T, Hirano H, et al. Comparison of physical properties of agar, low gel strength agar and gelatin, as supplementary food for people with swallowing difficulty. Journal of Texture Studies. 2002;33(4):285295. DOI: $10.1111 /$ j.1745-4603.2002. tb01350.x

[47] Ashida I, Iwamori H, Kawakami S, Miyaoka Y, Murayama A. Analysis of physiological parameters of masseter muscle activity during chewing of agars in healthy young males. Journal of Texture Studies. 2007;38(1):87-99. DOI: 10.1111/j.1745-4603.2007.00087.x

[48] Ashida I, Iwamori H, Kawakami S, Miyaoka Y, Murayama A. Analysis of the pattern of suprahyoid muscle activity during pharyngeal swallowing of foods by healthy young subjects. 
Journal of Medical Engineering \&

Technology. 2010;34(4):268-273. DOI: $10.3109 / 03091901003646096$

[49] Dantas RO, Dodds WJ, Massey BT, Kern MK. The effect of high- vs low-density barium preparations on the quantitative features of swallowing. AJR. American Journal of Roentgenology. 1989;153(6):1191-1195. DOI: 10.2214/ajr.153.6.1191

[50] Dantas RO, Kern MK, Massey BT, Dodds WJ, Kahrilas PJ, Brasseur JG, et al. Effect of swallowed bolus variables on oral and pharyngeal phases of swallowing. The American Journal of Physiology. 1990;258(5 Pt 1):G675-G681. DOI: 10.1152/ajpgi.1990.258.5.G675

[51] Inagaki D, Miyaoka Y, Ashida I, Ueda K, Yamada Y. Influences of body posture on duration of oral swallowing in normal young adults. Journal of Oral Rehabilitation. 2007;34(6):414-421. DOI: 10.1111/j.1365-2842.2007.01737.x

[52] Inagaki D, Miyaoka Y, Ashida I, Yamada Y. Influence of food properties and body posture on durations of swallowing-related muscle activities. Journal of Oral Rehabilitation. 2008;35(9):656-663. DOI: 10.1111/j.1365-2842.2008.01866.x

[53] Inagaki D, Miyaoka Y, Ashida I, Yamada Y. Influence of food properties and body position on swallowing-related muscle activity amplitude. Journal of Oral Rehabilitation. 2009;36(3). DOI: 10.1111/j.1365-2842.2008.01927.x

[54] Lowe AA. The neural regulation of tongue movements. Progress in Neurobiology. 1980;15(4):295-344. DOI: 10.1016/0301-0082(80)90008-8

[55] Inagaki D, Miyaoka Y, Ashida I, Yamada Y. Activity pattern of swallowing-related muscles, food properties and body position in normal humans. Journal of Oral
Rehabilitation. 2009;36(10):176-183.

DOI: 10.1111/j.1365-2842.2009.01994.x

[56] Sakamoto H, Harada T, Matsukubo T, Takaesu Y, Tazaki M. Electromyographic measurement of textural changes of foodstuffs during chewing. Agricultural and Biological Chemistry. 1989;53(9):2421-2433. DOI: 10.1271/bbb1961.53.2421

[57] Alfonso M, Neyraud E, Blanc O, Peyron MA, Dransfield E. Relationship between taste and chewing patterns of visco-elastic model foods. Journal of Sensory Studies. 2002;17(2):193206. DOI: 10.1111/j.1745-459X.2002. tb00342.x

[58] Miyaoka Y, Ashida I, Tamaki Y, Kawakami S, Iwamori H, Yamazaki $\mathrm{T}$, et al. Analysis of masseter activity patterns using $\mathrm{Tp}$ values during chewing of foods with different shapes and textural properties. Journal of Texture Studies. 2013;44(3):196-204. DOI: 10.1111/jtxs.12012

[59] Miyaoka Y, Ashida I, Kawakami S, Tamaki Y, Miyaoka S. Activity patterns of the suprahyoid muscles during swallowing of different fluid volumes. Journal of Oral Rehabilitation. 2010;37(8):575-582. DOI: 10.1111/j.1365-2842.2010.02081.x

[60] Delcomyn F. Neural basis of rhythmic behavior in animals. Science. 1980;210(4469):492-498

[61] Koizumi H, Nomura K, Yokota Y, Enomoto A, Yamanishi T, Iida S, et al. Regulation of trigeminal respiratory motor activity in the brainstem. Journal of Dental Research. 2009;88(11):1048-1053. DOI: $10.1177 / 0022034509345998$

[62] Yazawa I. Reciprocal functional interactions between the brainstem and the lower spinal cord. Frontiers in Neuroscience. 2014;8:124. DOI: 10.3389/ fnins.2014.00124 

Section 2

\section{Pathological Aspect of Dysphagia}





\title{
Chapter 2
}

\section{Pathology of Nonneoplastic Lesions of the Vocal Folds}

\author{
Nil Çomunoğlu, Şebnem Batur and Ayşe Mine Önenerk
}

\begin{abstract}
Nonneoplastic vocal fold lesions are common that can cause hoarseness and voice change. Reactive lesions of Reinke's space can be observed in all ages and genders and comprise the majority of the benign nonneoplastic vocal fold lesions. Although clinically different terms are used to define reactive lesions of Reinke's space, they share the same histopathologic features. In order to differentiate vocal fold polyp and nodule and Reinke's edema, clinical findings should be considered. Epithelial changes such as pseudoepitheliomatous and verrucous hyperplasia may cause diagnostic challenge due to resemblance of squamous cell carcinoma. Evaluation of the invasion border and cellular atypia may aid in correct diagnosis.
\end{abstract}

Keywords: vocal fold nodule, vocal fold polyp, Reinke’s edema, contact ulcer, keratosis

\section{Introduction}

The vocal folds, also known as vocal cords, extend through the laryngeal cavity bilaterally and are primarily responsible for voice production. The thyroarytenoid muscle, deep lamina propria, intermediate lamina propria, superficial lamina propria, and squamous epithelium comprise the vocal folds [1-3]. The superficial lamina propria, which is also called Reinke's space, consists of loose fibrous or elastic components and provides a gelatinous surface for vocal folds to vibrate upon. The deep and superficial lamina propria forms the vocal ligament. Madruga et al. demonstrated that type I collagen is abundant in the superficial lamina propria which forms a narrow band. The intermediate lamina propria essentially contains type III collagen, and the deep lamina propria is rich from both type I and type III collagen [4].

In a study of Chandramouli et al., nonneoplastic lesions of the larynx comprise $80 \%$ of the benign lesions diagnosed [5]. The etiology of the nonneoplastic vocal fold lesions is usually multifactorial, including phonotrauma (excessive loudness and cough, excess tension while speaking or singing, etc.), laryngeal trauma (endotracheal intubation), hypothyroidism, cigarette smoking, alcohol abuse, and gastroesophageal reflux (GERD) [6-10].

\section{Exudative lesions of Reinke's space}

Vocal fold nodules and polyps and Reinke's edema are exudative vascular-stromal lesions involving Reinke's space. The similarities and distinct features of these lesions have long been studied [11]. Although some discriminating features have 
been emphasized, most of the literature concludes that these lesions share the same histopathologic features which prevent a definitive diagnosis [11, 12].

\subsection{Reinke’s edema}

Reinke's edema is one of the most common causes of hoarseness and approximately comprises $10 \%$ of the laryngeal pathologies $[13,14]$. It is the result of fluid accumulation in Reinke's space that lies beneath the surface epithelium of the true vocal cords [13]. Smoking, vocal abuse, upper respiratory tract infection, and gastroesophageal reflux (GERD) have been associated with Reinke's edema $[13,15-17]$. Marcotullio revealed that the occurrence and recurrence of Reinke's edema depend on the number of cigarettes daily used [15]. Zeitels et al. showed that increased subglottic aerodynamic driving pressure is the underlying mechanism of edema [18]. It has been hypothesized that vocal hyperfunction along with the underlying conditions such as reflux, smoking, etc. is more prone to Reinke's edema [13]. Vecerina et al. classified Reinke's edema into transparent and livid type [19]. Hypothyroidism is stated not to be related with Reinke's edema [13, 20-22]. Majority of the patients are adults (aged between 20 and 60 years). Most of the patients experience Reinke's edema unilaterally (74\%). Only a small group of the patients has bilateral edema (26\%) [13].

Most of the time, serous fluid is observed upon incision in macroscopic evaluation. However, if the condition persists longer, a jellylike fluid can be seen [13]. Patients are treated with vocal rehabilitation and surgery after eliminating the underlying conditions. Cessation of cigarette smoking is an important factor in long-term treatment $[23,24]$. Histopathologic features include subepithelial edema and expansion of the intercellular space, especially the basal membrane. Few blood vessels can be observed [25]. The overlying epithelium is normal most of the time [13]. Duflo et al. showed that antioxidant gene expression is increased in Reinke's edema compared to the vocal fold polyps [26]. In addition, Branski et al. demonstrated that heme oxygenase is increased in vocal fold fibroblasts [27]. Collagen and elastin fiber configuration alterations are demonstrated in Reinke's edema [28, 29]. Dikker et al.s stated that increased fibrin, hemorrhage, and thickening of the basement membrane are related with Reinke's edema [30].

\subsection{Vocal fold nodule and polyp}

Vocal fold nodules and polyps are polypoid lesions of the vocal folds sharing the same histopathologic features. They are usually differentiated by clinical findings. A polypoid lesion larger than $3 \mathrm{~mm}$ is often regarded as a polyp, whereas smaller lesions are classified as a nodule [31]. Vocal fold nodules are usually bilateral lesions involving anterior or middle vocal fold and located superficially to the free edge of the vocal fold [32]. They are slightly more common in women and in young age $[10,33]$. Vocal polyps occur more commonly at the anterior portion of the vocal fold, and in more than $90 \%$ of the patients, they are unilateral [31]. There is no age and gender predilection for vocal polyps. Vocal abuse is the leading etiologic factor for vocal fold nodules and polyps; singers, lecturers, and coaches are more prone to developing these lesions due to excessive and loud voice use $[6,10]$. Infection, hypothyroidism, GERD, cigarette smoking, and allergy are the other frequent causes $[7-9,34]$. Patients present with hoarseness and change in voice quality.

Grossly, vocal cord polyps and nodules present as sessile or polypoid lesions with nodules presenting as few millimeters and polyps up to few centimeters in size [5]. They can be white or bright red, and their consistency varies from firm to soft with a mucoid or glistening cut surface [32]. 
Although previous studies have attempted to identify histopathologic features which may aid in the differential diagnosis, it is now widely accepted that vocal fold nodules/polyps cannot be differentiated on the histopathologic basis [11, 31, 32, 35]. Epithelial hyperplasia, basement membrane thickening, edema, and vascular proliferation can be seen both in nodules/polyps and Reinke's edema [25, 31, 36]. Ancillary studies such as Verhoeff-van Gieson, Masson trichrome, and Alcian blue did not reveal any difference among these entities [31].

Histopathology represents primarily the extent of the vascular damage and the temporal stage of the lesion [37]. Vocal abuse causes vascular injury and increased vascular permeability. If the damage is minimal, then increased permeability causes only edema, and the microscopic evaluation reveals hypocellular myxoid stroma which is defined as edematous myxoid-type vocal fold nodule/polyp according to some authors (Figure 1). When this lesion is not removed or resolved, it undergoes fibrosis, and fibrous-type polyp/nodule evolves. Oval and spindle cells are observed embedded in a fibrous stroma (Figure 2). Rarely atypical cells can be detected in this type which is not related with an aggressive behavior [36, 38, 39]. However, if the vascular injury is severe enough for fibrin escape to the subepithelium and interstitium at the beginning of the injury, then hyaline-type polyp/nodule occurs which is characterized by a hypocellular/acellular eosinophilic stroma. By the time capillary proliferation occurs, it progresses to a vascular-type nodule/polyp. This subtype shows ectatic vascular channels in a hypocellular stroma (Figure 3). Although, these classification and staging are helpful in understanding the

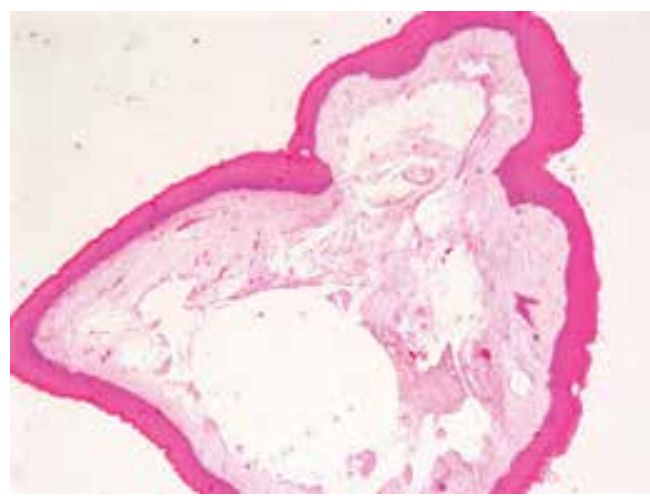

Figure 1.

Polyp with hypocellular myxoid stroma (H\&E, ×100).

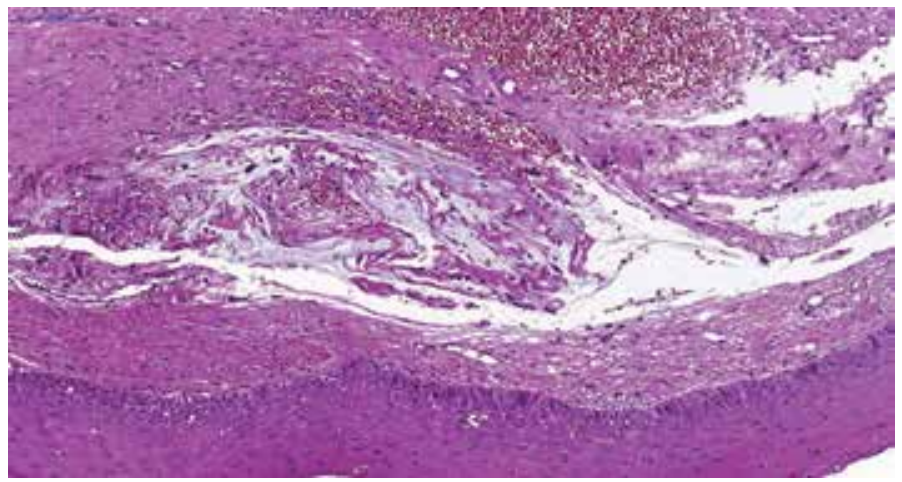

Figure 2.

Polyp stroma appears fibrotic, and spindle cells can be observed embedded within the stroma $(H \& E, \times 200)$. 


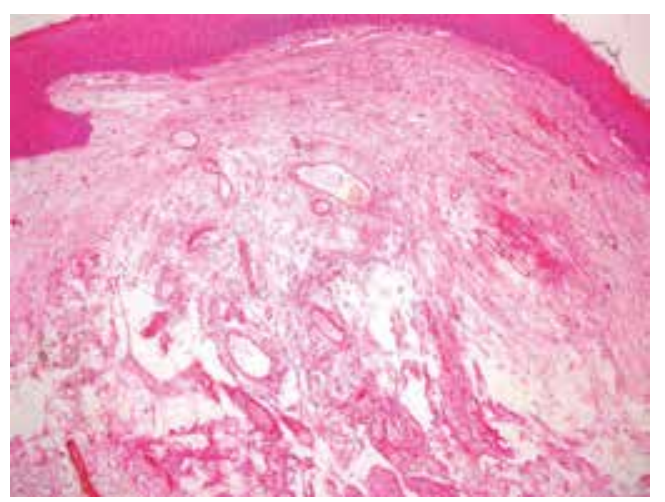

Figure 3.

Polyp stroma shows ectatic vasculature with a hypocellular stroma $(H \& E, \times 200)$.

histopathologic spectrum, it is clinically insignificant. In addition, mixed features are seen in a single lesion most of the time. The overlying squamous epithelium may be normal, atrophic, or hypertrophic, and keratosis can be seen.

Differential diagnosis includes amyloidosis, myxoma, and contact ulcer. Differentiation of hyaline-type vocal fold nodule/polyp from amyloidosis can be made by observing the distribution of eosinophilic material carefully. In hyaline-type nodule/polyp, it is more homogenous, whereas in amyloidosis there is perivascular and periglandular accentuation of the deposit. Histochemical stains like Congo red and crystal violet can be helpful in identifying the nature of the amorphous material. Myxoma has been rarely reported in the larynx, and it has characteristic stellate spindle cells embedded in an avascular, basophilic, gelatinouslike matrix $[40,41]$. Contact ulcer has an ulcerated and fibrin-coated surface and usually involves the posterior portion of the vocal folds.

Surgery has a limited value for these lesions as they are reactive changes to an underlying injurious condition which must be managed. Not surprisingly, voice therapy may result in resolution in most of the patients [42, 43]. Also, treatment of the exact underlying condition such as hypothyroidism can be helpful in the treatment of vocal fold polyps/nodules.

\section{Contact ulcer}

Contact ulcer is an ulcerated granulation tissue due to vocal abuse, endotracheal intubation, and GERD. Men are more commonly affected than women except that postintubation-related ulcers are more common in female patients. Clinically patients present with hoarseness, cough, dysphonia, habitual coughing, and throat cleaning [44].

Contact ulcers are generally bilateral ("kissing ulcer") and involve the posterior part of the vocal fold. Grossly they present as an ulcerated, tan-white to erythematous polypoid lesion up to $3 \mathrm{~cm}$ in diameter. Microscopic evaluation reveals ulceration underlying a fibrin and/or fibrinoid necrosis and capillary proliferation that is oriented perpendicularly to the mucosal surface (Figure 4). Central and base part of the lesion contains hemosiderin-laden macrophages. Usually a dense inflammatory infiltration accompanies. Re-epithelization, mucosal hyperplasia, and fibroblastic proliferation can be observed in chronic phase of the lesion [45].

Clinicopathologic correlation is important in the correct diagnosis as histopathologic features are somewhat nonspecific. Differential diagnosis includes vasculartype vocal cord polyp/nodule, Kaposi sarcoma, Wegener granulomatosis, and 


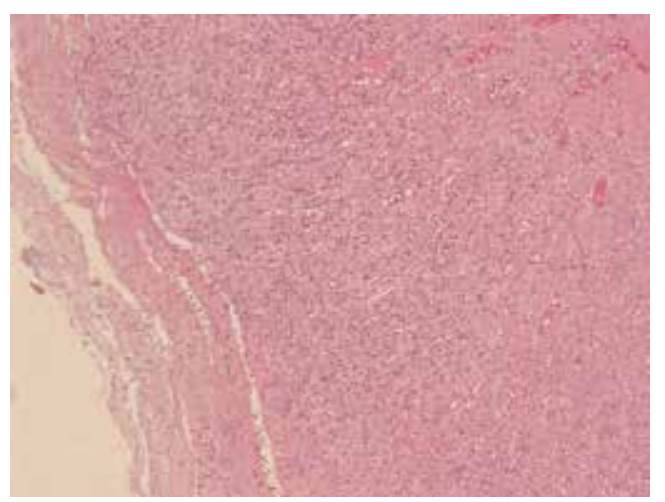

Figure 4.

Ulceration, necrosis, and granulation tissue formation. Capillaries oriented perpendicular to surface $(H \mathcal{E} E, \times 200)$.

infectious agents. Wegener granulomatosis can be differentiated from contact ulcer with features of vasculitis and necrotizing granulomas. Also, laboratory findings, such as cytoplasmic antineutrophil cytoplasmic antibody (c-ANCA) positivity, support a diagnosis of Wegener granulomatosis over contact ulcer. Kaposi sarcoma may rarely involve the larynx, and histopathologic evaluation reveals spindle cells with intracellular spheroid hyaline globules forming slit-like spaces and anastomosing vascular channels. Immunohistochemical staining with human herpes virus 8 (HHV8) reliably confirms the diagnosis as Kaposi sarcoma.

Like vocal polyps/nodules, contact ulcer is treated with voice therapy, antiGERD medication, and behavioral modifications in order to decrease habitual coughing and throat clearing [44].

\section{Amyloidosis}

Amyloidosis is the deposition of an extracellular, acellular, fibrillar, and amorphous material in various sites of the body. It can be primary (associated with plasma cell neoplasms) or secondary (associated with chronic systemic diseases such as familial Mediterranean fever, rheumatoid arthritis, etc.). Primary amyloidosis can be classified as systemic or localized. The larynx is the most common site for localized amyloidosis [46, 47]. It may also be associated with "mucosa-associated lymphoid tissue" (MALT) lymphoma or a neuroendocrine tumor. Laryngeal amyloidosis generally affects adults at the sixth decade. Patients present with hoarseness and voice changes because of mass effect [45]. Grossly it presents as a firm polypoid lesion up to $4 \mathrm{~cm}$ covered by normal-appearing mucosa. Cut surface may be firm and starch-like and gray or yellow-orange in color. Localized laryngeal amyloidosis can involve the larynx diffusely or present as a nodule. Microscopic examination reveals diffuse subepithelial or discrete nodular lesion consisting of amorphous, fibrillary, and hyaline-like eosinophilic substance deposition. Usually the distribution of the deposition is more pronounced in perivascular and periglandular areas. Lymphoplasmacytic infiltration and foreign body giant cells can be seen but usually sparse.

Differential diagnosis includes hyalinized-type vocal fold polyps/nodules and lipoid proteinosis which are negative with Congo red and crystal violet. Most importantly as amyloid can be associated with multiple myeloma, laryngeal neuroendocrine tumors, and medullary thyroid carcinoma, a systemic evaluation of the patient is necessary. 
If amyloidosis is limited to the larynx, patients are treated with surgical removal of the lesion. However, other treatment options will be applied if the patient has an underlying neoplastic condition or chronic inflammatory disease.

\section{Cysts of the larynx}

Nonneoplastic cysts of the larynx consist of laryngocele, saccular, and ductal cysts [48-50].

\subsection{Laryngocele}

Laryngeal ventricles are the spaces between the true and false vocal cords which extend upward and form the laryngeal saccule bilaterally. Laryngocele can be defined as a symptomatic dilation of the laryngeal saccule with air entrapment [51]. An important feature of laryngocele is that the lumen of the cyst communicates with the laryngeal cavity. Radiography reveals an air-filled cystic enlargement. They are usually unilateral, may occur over a wide age range, and are more frequent in males. Occupations such as glassblower or wind instrumentalists are at increased risk of developing laryngocele due to repetitive increase in intralaryngeal pressure. Patients present with hoarseness and foreign body or globus sensation.

Clinically it can be divided as internal, external, or combined. Internal laryngocele is confined to the endolarynx and presents as a supraglottic submucosal lesion. In contrast external laryngocele extends through the thyrohyoid membrane. However, in combined laryngocele, the cyst herniates through the thyrohyoid membrane and presents as an anterior neck mass [51-53]. Patients present with hoarseness, dyspnea, and chronic cough. In histopathologic examination, the respiratory epithelium is identified as the lining of the cyst wall.

Differential diagnosis includes other laryngeal cysts. However, laryngocele is the only air-filled cyst of this region and communicates with the laryngeal cavity-an important finding in differentiation.

Symptoms may resolve with the expulsion of trapped air from the cyst lumen. Treatment of laryngocele is simple excision or marsupialization of the cyst wall.

\subsection{Saccular cyst}

Saccular cyst is a mucin-filled cyst due to obstruction of the laryngeal saccule. It can be acquired or congenital $[49,50]$. If it extends medially, it may obscure the anterior vocal fold. Lateral saccular cysts are similar to the external laryngoceles, herniated through the thyrohyoid membrane, and may present as a neck mass [37].

Saccular cysts are usually lined by respiratory-type epithelium. Squamous or oncocytic lining can be observed in some cases. Cyst lumen is filled with mucin or acute inflammatory exudate.

Differential diagnosis includes laryngocele and thyroglossal ductus cyst. It is differentiated from laryngocele by mucin content, and it does not communicate with the laryngeal cavity. It is difficult to discriminate a thyroglossal cyst from a saccular cyst when it invades through the pre-epiglottic space and histopathologically lacks the thyroid tissue. When this is the situation, investigation of the anatomic localization of the cyst is a reliable finding in the differentiation of these cysts. Thyroglossal ductus cysts are related with the hyoid bone and located in the midline of the neck. 


\subsection{Ductal cysts (squamous, tonsillar, oncocytic)}

Ductal cysts are the most common type of laryngeal cysts. Cyst lining epithelium can be squamous or oncocytic $[49,54]$ (Figures 5 and 6 ). When a squamous lined cyst is surrounded by lymphoid tissue, it is called a tonsillar cyst. Tonsilar cysts are more common in the vallecula which contains tonsillar remnants.

\subsection{Other cysts}

Rarely epidermal, dermoid, and branchial cleft cysts may occur in the endolarynx.

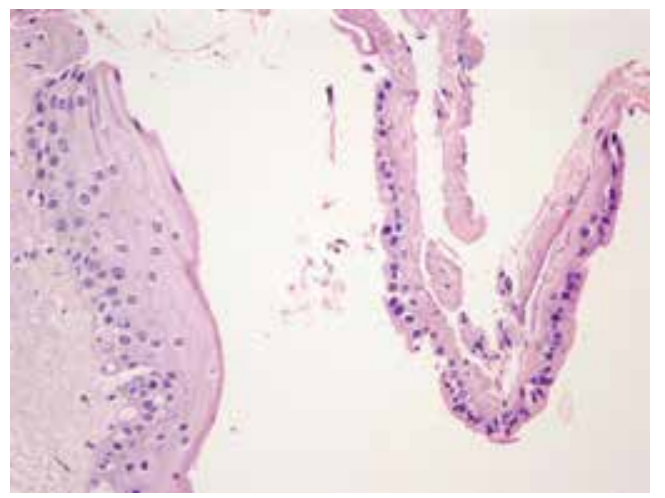

\section{Figure 5.}

The right side of the figure represents a squamous cell lined cyst. On the left cyst lining cells appear oncocytic (HङE, ×400).

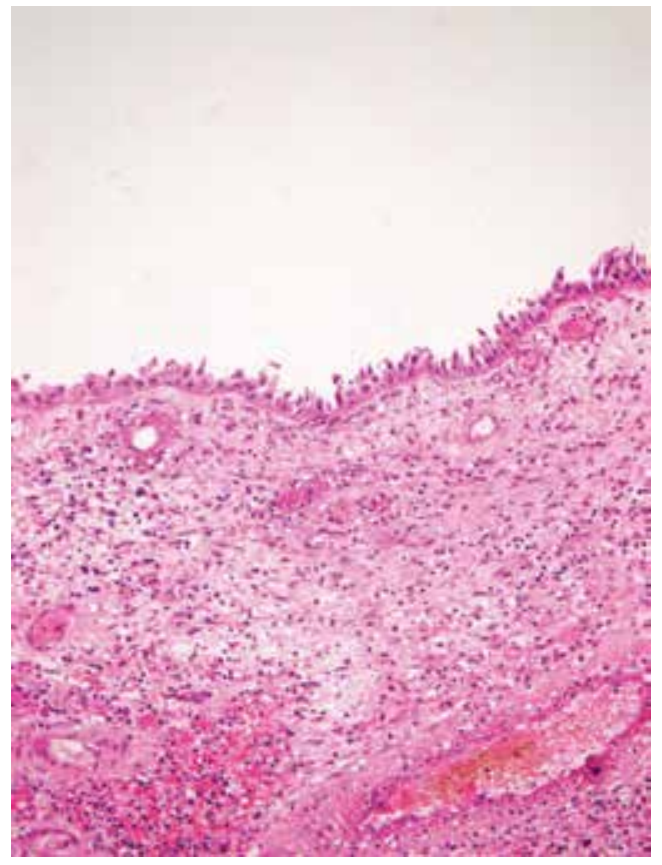

Figure 6.

Ductal cyst with lining oncocytic cells (HङE, ×400). 


\section{Hyperplastic lesions of the larynx}

Hyperplastic lesions of the larynx are a result of a reparative process. Clinical terms such as leukoplakia (white plaque), erythroplakia (red plaque), erythroleukoplakia (red and white changes), or pachydermia (extensive thickening of the mucosa) are used to describe the lesion macroscopically, and they are not histologic entities [55].

Keratosis of the larynx is synonymously used with simple hyperplasia and squamous hyperplasia [56]. Singers and other occupations that lead to an individual to use his/her voice excessively and cigarette smokers are at risk of keratosis. Hoarseness is the leading complaint. Laryngoscopy identifies leukoplakia. Microscopic evaluation reveals a thickened squamous epithelium without cellular or architectural atypia [55]. Generally, a hyperkeratotic layer overlies the epithelium. Lesions with warty configuration are referred as verrucous keratosis [56, 57].

Pseudoepitheliomatous hyperplasia $(\mathrm{PEH})$ is a reactive proliferation of the squamous epithelium which develops in response to infections, trauma, or neoplasia. Histologically these lesions are downward thickening of the epithelium (Figure 7). Due to a less well-defined epithelial stromal interface and the tendency of anastomosing epithelial tongues entrapping the submucosa, the lesion may mimic squamous cell carcinoma [58]. However, in $\mathrm{PEH}$, the hyperplastic epithelium forms large bulbous projections, and basement membrane is always preserved.

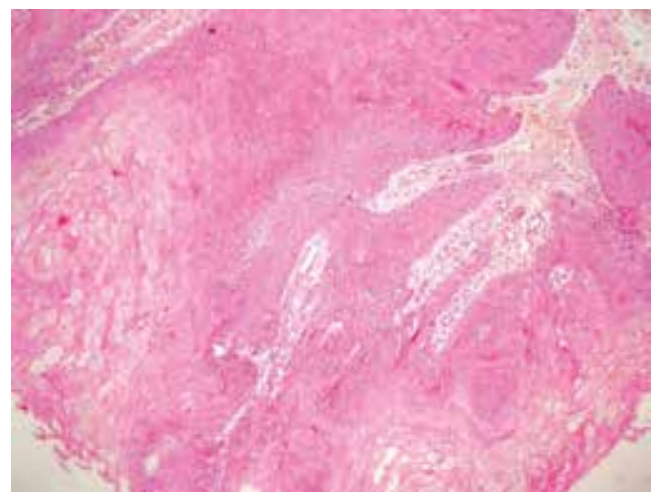

Figure 7.

Downward proliferation of benign appearing surface epithelium $(H \Theta E, \times 200)$.

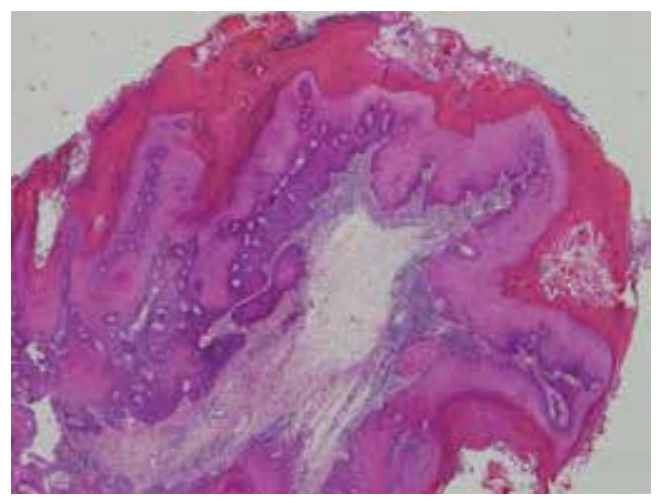

Figure 8.

Parakeratosis and verrucous hyperplasia of the surface epithelium (HङE, ×100). 
Verrucous hyperplasia is a verrucous and keratotic form of squamous hyperplasia which may show varying degrees of cytological atypia (Figure 8). The presence of cellular atypia and a relatively regular epithelial-stromal border supports a diagnosis of verrucous hyperplasia over verrucous squamous cell cancer (SCC) [59].

\section{Conclusions}

Voice change or hoarseness of voice and nonneoplastic vocal fold lesions lead to a high percentage of vocal fold biopsies. Both clinical and histopathologic features should be considered in order to differentiate lesions involving Reinke's space and also hyperplastic epithelial lesions from squamous cell carcinoma.

\section{Author details}

Nil Çomunoğlu ${ }^{1 *}$, Şebnem Batur ${ }^{1}$ and Ayşe Mine Önenerk ${ }^{2}$

1 Pathology Department, Istanbul University Cerrahpasa, Cerrahpasa Medical Faculty, Istanbul, Turkey

2 Pathology Department, Karaman State Hospital, Karaman, Turkey

*Address all correspondence to: nilcomunoglu@gmail.com

\section{IntechOpen}

(C) 2019 The Author(s). Licensee IntechOpen. This chapter is distributed under the terms of the Creative Commons Attribution License (http://creativecommons.org/licenses/ by/3.0), which permits unrestricted use, distribution, and reproduction in any medium, provided the original work is properly cited. (cc) BY 


\section{References}

[1] Thibeault SL. Advances in our understanding of the Reinke space. Current Opinion in Otolaryngology \& Head and Neck Surgery. 2005;13:148-151

[2] Volic SV, Klapan I, Seiwerth S, Ibrahimpasic T. Extracellular matrix of Reinke's space in some pathological conditions. Acta Oto-Laryngologica. 2004;124:505-508

[3] Claassen H, Werner JA. Fibre differentiation of the human laryngeal muscles using the inhibition reactivation myofibrillar ATPase technique. Anatomy and Embryology (Berlin). 1992;186:341-346

[4] Madruga de Melo EC, Lemos M, Aragão Ximenes Filho J, Sennes LU, Nascimento Saldiva PH, Tsuji DH. Distribution of collagen in the lamina propria of the human vocal fold. The Laryngoscope. 2003;113:2187-2191

[5] Chandramouli MP, Sengottuvelu P, Vivek B. Non-malignant lesions of larynx-Our experience. IOSR Journal of Dental and Medical Sciences (IOSRJDMS). 2018;17(2):54-57

[6] Bastian RW, Thomas JP. Do talkativeness and vocal loudness correlate with laryngeal pathology? A study of the vocal overdoer/underdoer continuum. Journal of Voice. 2016;30(5):557-562. DOI: 10.1016/j.jvoice.2015.06.012

[7] Chung JH, Tae K, Lee YS, Jeong JH, Cho SH, Kim KR, et al. The significance of laryngopharyngeal reflux in benign vocal mucosal lesions. Otolaryngology and Head and Neck Surgery.

2009;141:369-373

[8] Karkos PD, McCormick M. The etiology of vocal fold nodules in adults. Current Opinion in Otolaryngology \& Head and Neck Surgery. 2009;17(6):420-423. DOI: $10.1097 /$ MOO.0b013e328331a7f8
[9] Lechien JR, Saussez S, Nacci A, Barillari MR, Rodriguez A, Le Bon SD, et al. Association between laryngopharyngeal reflux and benign vocal fold lesions: A systematic review. The Laryngoscope. 2019;129(9):329-341

[10] Smolander S, Huttunen K. Voice problems experienced by Finnish comprehensive school teachers and realization of occupational health care. Logopedics, Phoniatrics, Vocology. 2006;31(4):166-171

[11] Hantzakos A, Remacle M, Dikkers FG, Degols JC, Delos M, Friedrich G, et al. Exudative lesions of Reinke's space: A terminology proposal. European Archives of Oto-RhinoLaryngology. 2009;266(6):869-878. DOI: $10.1007 / \mathrm{s} 00405-008-0863-\mathrm{x}$

[12] Oates J, Winkworth A. Current knowledge, controversies and future directions in hyperfunctional voice disorders. International Journal of Speech-Language Pathology. 2008;10(4):267-277

[13] Goswami S, Patra TK. A clinicopathological study of Reinke's oedema. Indian Journal of Otolaryngology and Head and Neck Surgery. 2003;55(3):160165. DOI: $10.1007 /$ BF02991943

[14] Fritzell B, Sundberg J, Strange-Ebbesen A. Pitch change after stripping oedematous vocal folds. Folia Phoniatrica et Logopaedica. 1982;34:29-32

[15] Marcotullio D, Magliulo G, Pezone T. Reinke's edema and risk factors: Clinical and histopathologic aspects. American Journal of Otolaryngology. 2002;23:81-84

[16] Myerson MC. Smoker's larynx: A clinical pathological entity. The Annals of Otology, Rhinology, and Laryngology. 1950;59:541-546 
[17] Koufman JA. Gastroesophageal reflux and voice disorders. In: Rubin JS, Sataloff RS, Korovin GS, Gould WJ, editors. Diagnosis and Treatment of Voice Disorders. New York: IgakuShoin; 1995. pp. 161-175

[18] Zeitels SM, Hillman RE, Bunting GW, Vaughn T. Reinke's edema: Phonatory mechanisms and management strategies. The Annals of Otology, Rhinology, and Laryngology. 1997;106:533-543

[19] Vecerina VS, Klrincic N, Markov D. Some morphological. Histological, cytological and histochemical aspects of Reinke's oedema. Acta OtoLaryngologica. 1996;116:322-324

[20] Lindeberg H, Felding JU, Sogaard H, Ilium P. Reinke's oedema and thyroid function: A prospective study in 43 patients. Clinical Otolaryngology. 1987;12(6):417-420

[21] Wedrychowicz B, Nijander D, Betkowski A, Jastrzebski J. Reinke's edema and thyroid hypofunction. Otolaryngology. 1992;46(6):538-542

[22] White A, Sire DW, Maran AG. Reinke's oedema and thyroid function. The Journal of Laryngology and Otology. 1991;105(4):291-292

[23] Zalesska-Krecicka M, Krecicki T, Cyganek P. A clinical study of Reinke's edema. Otolaryngologia Polska. 1994;47(2):153-157

[24] Hojslet EE, Moesgaard-Nielsen V, Karlsmose M. Smoking cessation in chronic Reinke's oedema. The Journal of Laryngology and Otology. 1990;104:626-628

[25] Dikkers FG, Nikkels PG. Lamina propria of the mucosa of benign lesions of the vocal folds. The Laryngoscope. 1999;109:1684-1689

[26] Duflo SM, Thibeault SL, Li W, Smith ME, Schade G, Hess MM.
Differential gene expression profiling of vocal fold polyps and Reinke's edema by complementary DNA microarray. The Annals of Otology, Rhinology, and Laryngology. 2006;115:703-714

[27] Branski RC, Barbieri SS, Weksler BB, et al. The effects of transforming growth factor-beta1 on human vocal fold fibroblasts. The Annals of Otology, Rhinology, and Laryngology. 2009;118:218-226

[28] Sakae FA, Imamura MD, Sennes L, Mauad T, Saldiva P, Tsuji DH. Disarrangement of collagen fibers in Reinke's edema. The Laryngoscope. 2008;118:1500-1503

[29] Sakae FA, Imamura R, Sennes LU, Tsuji DH, Mauad T, Saldiva PH. Elastic fibers in Reinke's edema. The Annals of Otology, Rhinology, and Laryngology. 2010;119(9):609-614

[30] Dikkers FG, Nikkels PG. Benign lesions of the vocal folds:

Histopathology and phonotrauma. The Annals of Otology, Rhinology, and Laryngology. 1995;104:698-703

[31] Wallis L, Jackson-Menaldi C, Holland W, Giraldo A. Vocal fold nodule vs vocal fold polyp: Answer from surgical pathologist and voice pathologist point of view. Journal of Voice. 2004;18(1):125-129

[32] Bohlender J. Diagnostic and therapeutic pitfalls in benign vocal fold diseases. GMS Current Topics in Otorhinolaryngology-Head and Neck Surgery. 2013;13(12):Doc1

[33] Mudd P, Noelke C. Vocal fold nodules in children. Current Opinion in Otolaryngology \& Head and Neck Surgery. 2018;26(6):426-430. DOI: 10.1097/MOO.0000000000000496

[34] Martins RH, Defaveri J, Domingues MA, de Albuquerque e Silva R. 
Vocal polyps: Clinical, morphological and immunohistochemical aspects. Journal of Voice. 2011;25(1):98-106. DOI: 10.1016/j.jvoice.2009.05.002

[35] Cipriani NA, Martin DE, Corey JP, Portugal L, Caballero N, Lester R, et al. The clinicopathologic spectrum of benign mass lesions of the vocal fold due to vocal abuse. International Journal of Surgical Pathology. 2011;19(5):583-587. DOI: $10.1177 / 1066896911411480$

[36] Loire R, Bouchayer M, Cornut G, Bastian RW. Pathology of benign vocal fold lesions. Ear, Nose, \& Throat Journal. 1988;67(5):357-358. 360-2

[37] Barnes L. Diseases of the larynx, hypopharynx, and trachea. In:

Surgical Pathology of the Head and Neck. 2nd ed. New York, Basel: Marcel Dekker; 2001. pp. 109-119

[38] Batsakis J. Stromal cell atypia in sinonasal polyposis. The Annals of Otology, Rhinology, and Laryngology. 1986;321-322(13):95

[39] Compagno J, Hyams V, Lepore M. Nasal polyposis with stromal atypia. Review of follow-up study of 14 cases. Archives of Pathology \& Laboratory Medicine. 1976;100:224-226

[40] Panda S, Kumar R, Gopinath VR, Sagar P. Head and neck myxoma presenting as isolated laryngeal polyp. Case Reports in Otolaryngology. 2018;6868737

[41] Val-Bernal JF, Martino M, Longarela MY. Cellular myxoma of the vocal cord: A case report and review of the literature. Turkish Journal of Pathology. 2017;13:1-5. DOI: 10.5146/ tjpath.2017.01417

[42] Hosoya M, Kobayashi R, Ishii T, Senarita M, Kuroda H, Misawa H, et al. Vocal hygiene education program reduces surgical interventions for benign vocal fold lesions: A randomized controlled trial. The Laryngoscope. 2018;128(11):2593-2599. DOI: $10.1002 /$ lary. 27415

[43] Mansuri B, Tohidast SA, Soltaninejad N, Kamali M, Ghelichi L, Azimi H. Nonmedical treatments of vocal fold nodules: A systematic review. Journal of Voice. 2018;32(5):609-620. DOI: 10.1016/j.jvoice.2017.08.023

[44] Emami AJ, Morrison M, Rammage L, Bosch D. Treatment of laryngeal contact ulcers and granulomas: A 12-year retrospective analysis. Journal of Voice. 1999;13(4):612-617

[45] Thompson LDR. Non-neoplastic lesions of the larynx, hypopharynx, and trachea. In: Thompson LDR, Goldblum JR, editors. Head and Neck Pathology. 2nd ed. Philadelphia:

Elsevier; 2013. pp. P107-P127

[46] Godbersen GS, Leh JF, Hansmann ML, Rudert H, Linke RP. Organ-limited laryngeal amyloid deposits: Clinical, morphological, and immunohistochemical results of five cases. The Annals of Otology, Rhinology, and Laryngology. 1992;101(9):770-775

[47] Berg AM, Troxler RF, Grillone G, Kasznica J, Kane K, Cohen AS, et al. Localized amyloidosis of the larynx: Evidence for light chain composition. The Annals of Otology, Rhinology, and Laryngology. 1993;102(11):884-889

[48] Canalis RF, Maxwell DS, Hemenway WG. Laryngocele-An updated review. The Journal of Otolaryngology. 1977;6(3):191-199

[49] DeSanto LW, Devine KD, Weiland LH. Cysts of the larynxClassification. The Laryngoscope. 2015;125(12):2629. DOI: 10.1002/ lary. 25563 
[50] Holinger LD, Barnes DR, Smid LJ, Holinger PH. Laryngocele and saccular cysts. The Annals of Otology, Rhinology, and Laryngology. 1978; 87(5 Pt 1):675-685

[51] Mobashir MK, Basha WM, Mohamed AE, Hassaan M, Anany AM. Laryngoceles: Concepts of diagnosis and management. Ear, Nose, \& Throat Journal. 2017;96(3):133-138

[52] Szwarc BJ, Kashima HK. Endoscopic management of a combined laryngocele. The Annals of Otology, Rhinology, and Laryngology. 1997;106:556-559

[53] Nazaroglu H, Ozates M, Uyar A, et al. Laryngopyocele: Signs on computed tomography. European Journal of Radiology. 2000;33(1):63-65

[54] Newman BH, Taxy JB, Laker HI. Laryngeal cysts in adults: A clinicopathologic study of 20 cases. American Journal of Clinical Pathology. 1984;81(6):715-720

[55] Ferlito A, Devaney KO, Woolgar JA, Slootweg PJ, Paleri V, Takes RP, et al. Squamous epithelial changes of the larynx: Diagnosis and therapy. Head \& Neck. 2012;34(12):1810-1816. DOI: 10.1002/hed.21862

[56] Rosai J. Larynx and Trachea. Surgical Pathology. 10th ed. Philadelphia: Elsevier; 2011. pp. 320-323

[57] Goodman ML. Keratosis (Leukoplakia) of the larynx. Otolaryngol Clinics of North America. 1984;17:179-183

[58] Gnepp DR. Diagnostic Surgical Pathology of the Head and Neck. 2nd ed. Philadelphia: Saunders Elsevier; 2009

[59] Stelow E. Larynx. In: Mills SE, editor. Diagnostic Surgical Pathology. 6th ed. Philadelphia: Wolters Kluwer; 2015. pp. P995-P1002 

Section 3

\section{Dysphagia in Stroke Patients}





\title{
Swallowing Disorders in Patients with Stroke
}

\author{
Aiko Osawa and Shinichiro Maeshima
}

\begin{abstract}
Swallowing disturbance often causes by stroke and may predispose patients to malnutrition and dehydration, as well as increases the risk of such complications as suffocation and aspiration pneumonia. As an initial evaluation, the water swallowing test can be easily carried out, but not all of the aspiration can be excluded. Therefore, videofluorography (VF) and videoendoscopic examination (VE) of swallowing should be performed to find a safety method of oral intake for providing visualization of the pharynx and larynx dysfunction. Clinical severity scale is important because once the severity is determined, the treatment strategy is also known. Swallowing training can be divided into indirect training without food (basic training) and direct training with food (eating training). In general, it is important to select conditions and training diets that are easy to swallow and have a low risk of aspiration while using indirect training and direct training that aim at gradually improving the level of oral intake.
\end{abstract}

Keywords: stroke, dysphagia, swallowing assessment, pneumonia, videofluorography

\section{Introduction}

Stroke is a group of diseases associated with sudden disturbance of consciousness and motor or cognitive dysfunctions induced by cerebrovascular disorder. Pathological conditions are roughly classified into three categories according to the causes: stroke occurred due to cerebrovascular occlusion, stroke induced by rupture of small cerebral vessels, and subarachnoid hemorrhage associated with rupture of brain aneurysm, etc. According to the reports of the National Stroke Association, incidence of stroke is one person every $40 \mathrm{~s}$; about 800,000 and 290,000 persons develop stroke every year in the USA [1] and Japan [2], respectively.

Medical management for stroke has changed remarkably in many ways such as rearrangement of emergency medical system, improvement of treatment methods including thrombolytic therapy and endovascular treatment of the brain, formation of medical teams in a stroke unit, and preparation of guidelines for stroke treatment, resulting in improvement of survival rate [3, 4]. More than a half of the survived patients however suffer from disabilities to a certain extent such as motor paralysis, sensory or visual impairment, cognitive disturbances including aphasia and unilateral neglect, and dysphagia, which all affect the patients' activities of daily living. 


\section{Incidence and features of dysphagia due to stroke}

It has been reported that dysphagia is observed in $39-81 \%$ of stroke patients during the acute phase $[5,6]$, and the results obtained from videofluoroscopic examination of swallowing (VF) or videoendoscopic examination of swallowing (VE) may reveal that even more patients are complicated by dysphagia [7]. However, it improves promptly, most of the time, in a few days or few weeks after the onset, and sever disorders may persist until the chronic stage in about $10 \%$ of the patients [7-9]. These show how important it is, for management of stroke, to prevent aspiration pneumonia and let patients regain ability to swallow safely during the acute phase.

Pathology of dysphagia varies according to the sites of the central nervous system being affected $[7,10]$. The cause of dysphagia after stroke is roughly categorized into two mechanisms: pseudobulbar palsy associated with disturbance of upper motor neurons toward nuclei in the medulla oblongata and bulbar palsy associated with lower motor neurons from nuclei in the medulla oblongata in the brain stem. In patients with pseudobulbar palsy induced by cerebrovascular events in cerebral lesions, symptoms and signs such as delayed swallowing reflex during the pharyngeal phase of swallowing, reduced laryngeal elevation, and residual food in the vallecula or pyriform sinus are observed. While in patients with bulbar palsy induced by brain stem lesions, loss of swallowing reflex and insufficient opening of the esophageal orifice are observed. In either case, patients are often complicated by disturbance of consciousness or cognitive disorder, and swallowing is also affected during the anticipatory, preparatory, and oral phases as described below.

\section{Swallowing process model}

There are two major systematic models known for normal swallowing dynamics (Figure 1). The first one is liquid swallowing model which consists of five phases including the anticipatory phase in addition to four other stages: preparatory, oral,

Five Stage Model for drinking and swallowing liquid

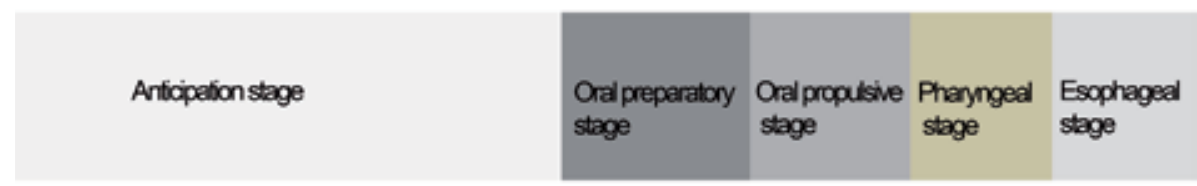

Process Model for eating and swallowing solid food

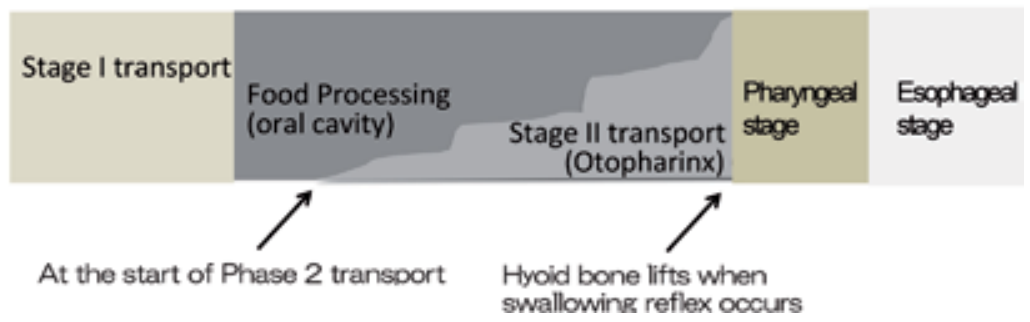

Figure 1.

Five stage and process models for swallowing. Two paradigmatic models are commonly used to describe the physiology of normal eating and swallowing: the five-stage model for drinking and swallowing liquid and the process model for eating and swallowing solid food. 
pharyngeal, and esophageal phases [11]. During the anticipatory phase (recognition phase), characteristics of food are recognized, the posture and the way to eat are determined, and saliva secretion is induced. During the preparatory phase, mastication occurs to make the food easy to swallow (into a food bolus). During the oral phase, the food bolus is transferred to the pharynx by the tongue movement. The pharyngeal phase begins when the food bolus formed in the vallecula starts moving downward through the pharyngeal cavity until it reaches the esophageal orifice. This is an involuntary action which occurs unconsciously. Contraction of the suprahyoid muscle and thyrohyoid muscle induces elevation of the hyoid bone and larynx. The epiglottis descends and blocks the laryngeal aperture to prevent aspiration of the food. Along with contraction of the pharyngeal muscles, the food bolus is sent to the esophageal orifice. Once the food bolus enters the esophagus during the esophageal phase, the cricopharyngeus muscle (upper esophageal sphincter) contracts to close the esophageal orifice so that food reflux will not occur.

The second one is a process model which involves mastication [12, 13]. Solid food is transported to a molar region by the tongue (Stage I transport). The food is grounded by mastication and transported actively to the oropharynx with the tongue movement (Stage II transport). Then, a food bolus is formed. Food transport occurs repeatedly even during mastication. The bolus is formed inside the oral cavity if it is command swallow of liquid food. However, if it is chew-swallow of solid food, the bolus is formed in the area between the oropharynx and the vallecula. If the bolus contains liquid, some of it reaches the pyriform sinus before swallowing reflex begins. Thus, it is important to understand that there are two different types of swallowing dynamics before making assessments.

\section{Assessment of dysphagia}

In assessment of dysphagia, the cause and onset mechanism of the condition should be revealed to understand pathology. The physical findings include observation of the level of consciousness and respiratory conditions. Neurological findings (especially on trigeminal, facial, glossopharyngeal, vagus and hypoglossal nerves), the level of cognitive functions, nutritional state, dehydration status, as well as the current status and past history of pneumonia, should be assessed. In addition, food residues inside the oral cavity, furred tongue, dental caries, characteristics of saliva, denture, and gingivitis should also be examined.

There is a risk of food aspiration whenever food or fluid is put inside the mouth. Therefore, assessment of swallowing functions should be performed if possible before ingesting food. Even if meals have already been started, ask patients and families detailed questions whether or not the patients have symptoms associated with aspiration.

\subsection{Interviews and observational assessments}

Characteristics of dysphagia are likely to be observed in the activities of daily living. Dysphagia can be suspected by asking simple questions to patients and their families. The question items may be associated with increase of cough and sputum after meals, sputum containing food, change in voice quality after meals, pharyngeal discomfort, decreased appetite, tired feeling during meals, prolongation of meal time (over $45 \mathrm{~min}$ ), change in the type of meals, and the way to eat, loss of weight, and fever. 


\subsubsection{Eating assessment tool (EAT-10)}

This is a standardized questionnaire form which is easy to use [14]. The assessment using a questionnaire form consists of 10 questions regarding pleasure of eating meals, loss of weight, effort of swallowing, etc. which are evaluated by scores between 0 (no problem) and 4 (serious problem). The maximum score is 40 .

\subsubsection{Mann assessment of swallowing ability (MASA)}

This is a highly reliable and valid assessment performed in stroke patients during the acute phase based on psychostatistic evaluations on consciousness, cooperative actions, auditory comprehension, etc. [15]. It consists of 24 items including alertness, cooperation, auditory comprehension, respiration, dyspraxia, saliva, and tongue movement, and the maximum score is 200 . Allocation of scores varies according to the level of importance for each item. The risk for dysphagia or aspiration and the degree of dysphagia are evaluated by the total score to determine the food form to be recommended. No special device is required, and the assessment can be performed within 15-20 min.

\subsection{Bedside swallowing assessment}

The swallowing assessments are performed at the bedside after confirming that the level of alertness is satisfactory $(<10)$ and that the patient's general condition is stable without any progression of stroke.

\subsubsection{Dry swallowing}

It is a basic movement to swallow saliva, demonstrating whether or not the patient is able to swallow before conducting a screening test [16].

\subsubsection{Repetitive saliva swallowing test (RSST)}

In this test, an examiner asks a patient to swallow saliva while palpating the hyoid bone and thyroid cartilage with the index finger and the middle finger, respectively, and counts how many times the patient swallows within $30 \mathrm{~s}[17,18]$. It is considered positive when $<3$.

\subsubsection{Water swallowing test (WST)}

The examiner asks a patient to swallow $30 \mathrm{ml}$ of water at the room temperature as usual and observes how many times he/she swallows and if he/she ever gets choked [19]. It is considered positive if the patient could swallow at once without getting choked within $5 \mathrm{~s}$.

\subsubsection{Modified water swallowing test (MWST)}

The examiner asks a patient to swallow $3 \mathrm{ml}$ of cold water and observes if he/ she ever gets choked and if the voice or respiratory state changes. The maximum score is 5 for this test $[18,20]$. If possible, the examiner asks the patient to swallow twice more. The test can be performed up to three times, and the worst swallowing activity is evaluated. It is considered abnormal when the score is $<3$ (swallowing present, respiration satisfactory, choking present, and/or wet hoarseness). 


\subsubsection{Food test (FT)}

In this test, the examiner asks a patient to ingest a tea spoon of flan (about $4 \mathrm{~g}$ ) $[18,21]$. The content of the assessment is the same as in MWST except that there is another assessment of residual food inside the oral cavity after swallowing in this test.

\subsubsection{Simple swallowing provocation test (SSPT)}

The examiner inserts a transnasal catheter 5 Fr into the epipharynx in the supine position and injects distilled water at room temperature to induce swallowing [22]. At first, the examiner injects $0.4 \mathrm{ml}$ of distilled water and observes for $3 \mathrm{~s}$. If the patient does not swallow, $2 \mathrm{ml}$ is additionally injected. If no swallowing was observed within $3 \mathrm{~s}$, it is considered that the patient has a risk of aspiration pneumonia.

\subsection{Comprehensive clinical two-step swallowing assessment scales}

There are existing clinical assessment scales which combined clinical symptoms and WST as below. Comprehensive clinical evaluations rather than simple WSTs demonstrate higher sensitivity and specificity.

\subsubsection{Two-step thickened water test (TTWT)}

With this method proposed by Smithard et al. [23], WSTs of $5 \mathrm{ml}$ (for three times) and $30 \mathrm{ml}$ are performed after obtaining clinical findings such as the alert level, trunk control, soft palate movement, and voluntary coughing.

\subsubsection{Toronto Bedside Swallowing Screening Test (TOR-BSST)}

This is a comprehensive WST focusing on evaluation of dysphagia of the oropharynx in addition to assessment of three items: voice quality, tongue movement, and pharyngeal sensation [24].

\subsubsection{Gugging swallowing screen (GUSS)}

Prior to the assessment, posture control is evaluated. An examiner assesses clinical findings including the state of consciousness, coughs (wet hoarseness), and dry swallowing (saliva swallowing) (5 points), followed by assessments of the swallowing state, presence or absence of choking or drooling, and change of voice after ingesting three food forms: semisolid, liquid, and solid (15 points) [25]. The food

form is determined according to the score (20, normal food; 15-19, soft food; 10-14, dysphagia food; 0-9, indirect training).

All these tests can be easily performed to assess swallowing functions. However, not all aspirations can be detected. Simultaneous assessments using VF and WST or FT under the X-ray [26, 27] revealed that the higher the amount of fluid, the more aspirations are observed with VF, while the detection rates for choking, coughing, and wet hoarseness were not that high (Figure 2). In patients with decreased cognitive or physical functions and patients with brain stem lesions, silent aspiration cannot be denied even if there is no clinical finding of choking, coughing, or wet hoarseness [28]. VF and VE should be performed as much as possible in case where dysphagia is strongly suspected in physical examinations or BSA or when there is no effective strategy established for the treatment. 


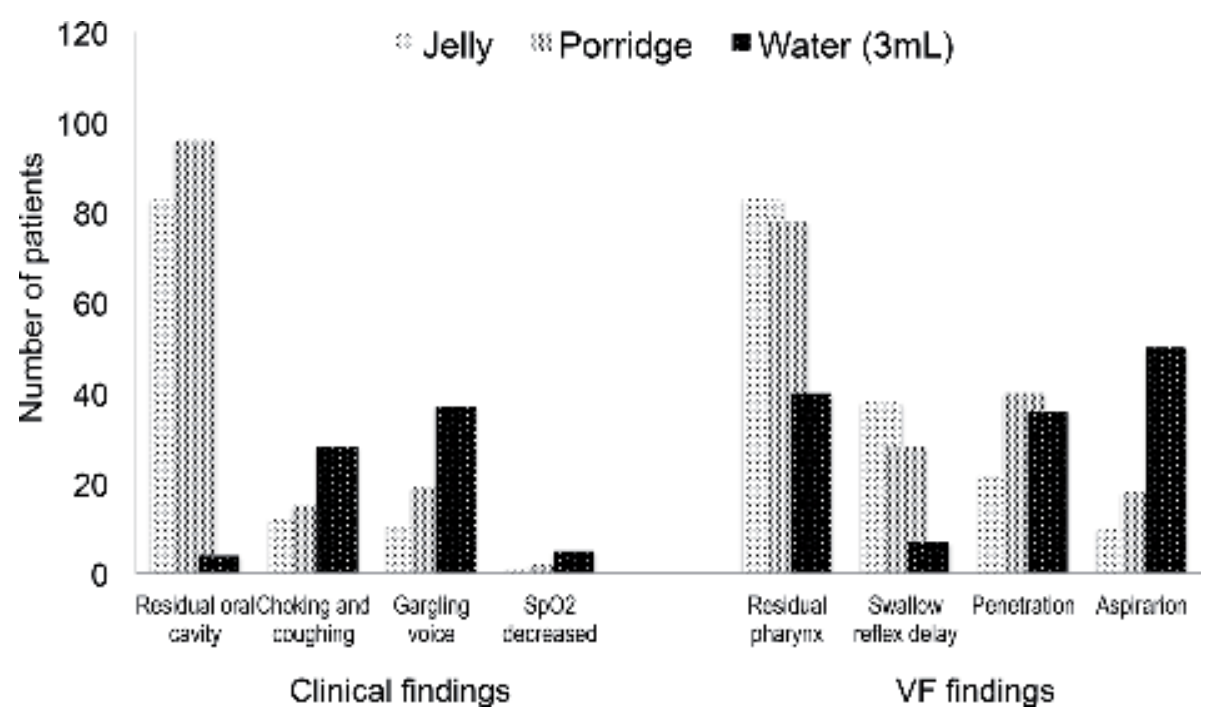

Figure 2.

Clinical and VF findings of the food test. Simultaneous assessments using VF and WST or FT under the X-ray revealed that the higher the amount of fluid, the more aspirations are observed with VF, while the detection rates for choking, coughing, and wet hoarseness were not that high.

\subsection{Videoendoscopy (VE) and videofluorography (VF)}

\subsubsection{Videoendoscopy (VE)}

Using a laryngeal fiberscope, the pharynx and larynx are primarily observed using real food (Figure 3). Although the moment of swallowing cannot be observed, early pharyngeal penetration of saliva or food bolus, timing of inducing swallowing reflex, pharyngeal residue, and laryngeal penetration/aspiration can be directly observed. This is a useful method which enables observation of the pharynx and larynx using actual food without irradiation. This can be used at the bedside as it does not require any large-scale devices [29].

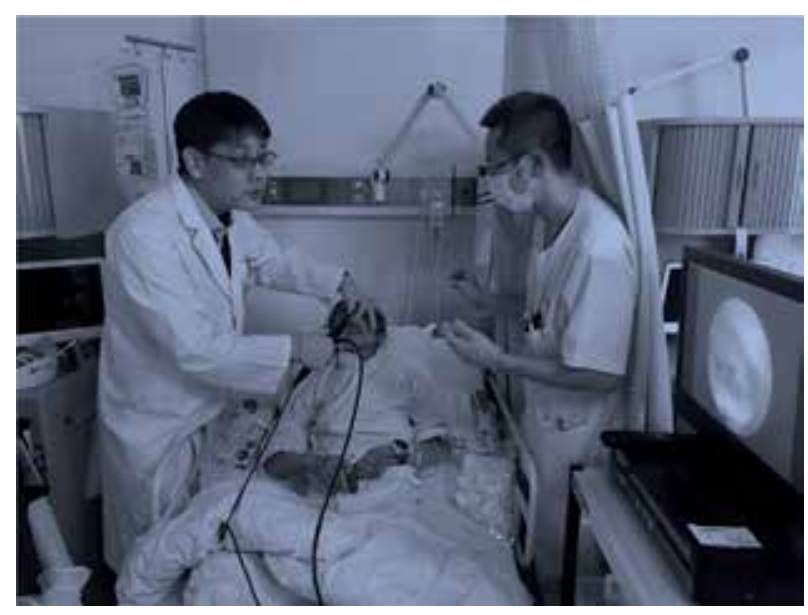

Figure 3.

Videoendoscopy (VE). Videoendoscopy can be performed on patients at bedside. 
VE can be performed without irradiation and is highly portable. In addition, it enables direct observation of mucosal and saliva conditions as well as assessment of actual meal settings. However, there is a risk of inducing pain or nasal bleeding when inserting the fiber, and it is difficult to observe oral cavity during mastication, bolus formation, and food transportation to the proximal part of the tongue; it becomes blurry at the moment of swallowing; hence, it is difficult to understand the details of laryngeal penetration/aspiration.

\subsubsection{Videofluorography (VF)}

Swallowing functions are assessed using an X-ray device. With this method, a patient is asked to ingest fluid (water) or solid/semisolid food (imitation food) which contain contrast medium (barium sulfate), and movement of the oral cavity, pharynx, and esophagus as well as structural abnormalities and movement of the food bolus are assessed [30]. The diagnostic value of this test is high as it can reveal formation of the food bolus and its transportation to the pharynx in the oral phase, the timing and the level of laryngeal elevation in the pharynx phase, and bolus passing at the esophageal orifice and the presence/absence or the level of aspiration (Figure 4). It also clarifies conditions where the risk of aspiration and pharyngeal residue is low and determines the safest food form, the method of ingestion, and the posture. Moreover, it also enables us to try various compensation methods and make detailed observations on dysphagia. Hence, this test should be considered as a gold-standard swallowing function assessment which can provide very important information to establish treatment strategy according to the findings of the observation [30].

\subsubsection{Swallowing CTscan}

With excellent spatial resolution $(0.5 \mathrm{~mm}$ square $)$ and time resolution (10 frames per second), 320 Area Detector CT (320-ADCT) enables us to precisely and quantitatively observe the swallowing movement in 3D. It presents 3D images of various organs from arbitrary directions; hence, it can precisely quantify swallowing dynamics [31, 32].

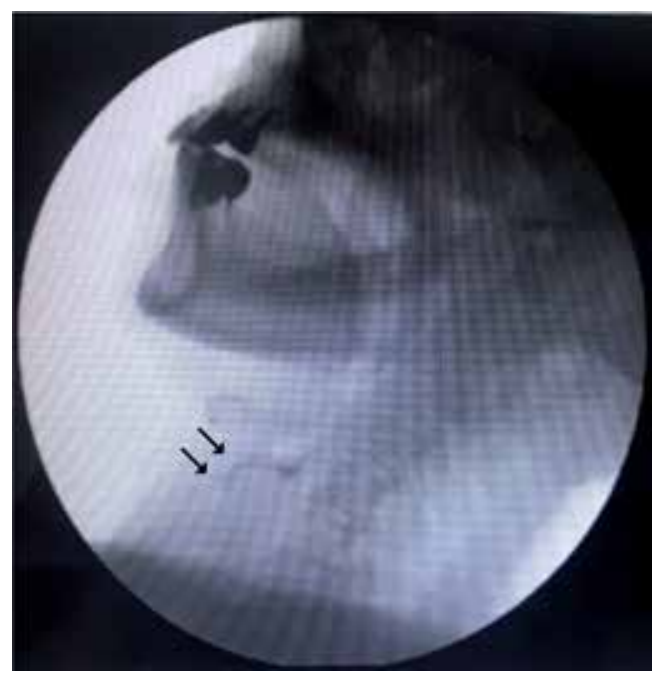

Figure 4.

Videofluorography (VF). VF shows that fluid is getting into the trachea from the larynx. 


\subsection{Assessment of severity in swallowing}

Taking into consideration the functional diagnosis, causative disease, and general condition of the patient, the level of feeding is determined as the final overall evaluation of swallowing. Swallowing Performance Scale [33] and Functional Oral Intake Scale (FOIS) [34] are seven-level scales used in overseas countries to assess oral feeding status during the meals. While in Japan, Dysphagia Severity Scale (DSS) [35] is often used. This is a comprehensive assessment using the ordinal scale of seven levels, which can suggest a management method to a certain extent if the severity level is determined. VF and VE are not necessarily required in an evaluation using DSS. An evaluation made on clinical findings or a screening test by a highly experienced nurse can be equivalent to the evaluation made on DSS using VE [36]. Dysphagia Outcome and Severity Scale (DOSS) is a simple and easy-to-use point scale developed only to systematically evaluate the functional level of severity. With this scale, recommendations on feeding levels can be prepared based on objective evaluations [37].

\section{Treatment of dysphagia}

There are two types of dysphagia rehabilitation: indirect training which does not require feeding and direct training which requires feeding. For a patient with severe dysphagia, rehabilitation should be started with the indirect training, and then the direct training can be added according to the progress. Likewise, for a patient who can start rehabilitation with the direct training, the indirect training may be added if necessary $[38,39]$.

\subsection{Indirect training}

Indirect trainings which are typically used include pharyngeal cooling stimulation (ice massage), Mendelsohn maneuver with which the upper esophageal sphincter is mechanically opened by keeping the positions of the larynx and the hyoid bone elevated, blowing, supraglottic swallow (swallow while holding breath), Shaker exercise (head lift exercise), cervical anterior flexion exercise, balloon dilatation method, and pharyngeal electrical stimulation. At the same time, it is essential to conduct oral care. If a patient does not ingest food, the amount of saliva secretion decreases, and as a consequence, self-cleansing action of oral cavity with saliva decreases as well. In such cases, patients often have dry mouth covered by biofilm as seen with the furred tongue, which need to be removed by brushing. These trainings are conducted primarily by speech-language-hearing therapists and nurses. However, the concomitant use of pulmonary respiration therapy conducted by physiotherapists or trainings to improve the ability of performing daily activities conducted by occupational therapists is also very effective to prevent/relieve aspiration pneumonia and improve feeding ability. Hence, it is important to treat patients with the entire team in the rehabilitation approach for dysphagia.

\subsection{Direct training}

In the direct training, feeding is started as soon as safety conditions for feeding were established based on detailed evaluations. The patient is observed for 3-7 days to check if there is any sign of aspiration or pneumonia, and the meal condition is upgraded accordingly. In stroke patients, swallowing functions are likely to change 
according to the alert level, and facial palsy is frequently observed. As their masticatory functions have been generally decreased due to facial and lingual palsy, the patients are unable to produce a smooth and appropriately sized food bolus with adequate mastication. The poor pharyngeal clearance is likely to induce formation of food residues inside the pharynx. As the residual food may cause aspiration or suffocation, it is dangerous to offer meals which require mastication to patients with stroke in the acute phase. Therefore, it is considered safer to start feeding with a single food type such as food paste in patients suspected to have dysphagia, even if it is mild.

Once the feeding started, special attention should be paid on increase of saliva/ drooling and sputum, increase of coughs, tiredness after eating meals, fever, voice change (wet hoarseness) during meals, and residual food inside the oral cavity. Aspiration can occur without choking. Therefore, if there is any sign as listed above, auscultation of the chest and vital sign assessment should be performed along with chest X-ray and blood collection, if necessary. In addition, chest CT scan should also be considered if the patient is suspected to have pneumonia in the posterior side of the lung as it is often difficult to obtain remarkable findings on chest X-ray in such cases. If aspiration pneumonia is detected in these tests, direct training should be suspended for a moment, and pneumonia should be treated immediately. Once the treatment is completed, swallowing function assessments should be performed at the bedside along with VE and VE, if necessary, to reevaluate the conditions. Special attention is required especially in elderly patients with bilateral lesions who have neurological manifestations as they are likely to develop aspiration pneumonia $[40,41]$.

\section{Conclusions}

In assessment and treatment of dysphagia, "not choking" and "safe feeding" are not necessarily the same in meaning. It is necessary to conduct the swallowing function assessments and understand the patients' conditions along with the level of consciousness and physical state. Safe feeding can be achieved in stroke patients only by selecting appropriate food materials according to their pathological conditions at the optimal timing with appropriate feeding posture and efforts.

\section{Conflict of interest}

The authors declare that they have no competing interests. 


\section{Author details}

Aiko Osawa ${ }^{1}$ and Shinichiro Maeshima ${ }^{2 *}$

1 National Center for Geriatrics and Gerontology, Obu, Japan

2 Kinjo University, Hakusan, Japan

*Address all correspondence to: shinichiromaeshima@gmail.com

\section{IntechOpen}

(C) 2019 The Author(s). Licensee IntechOpen. This chapter is distributed under the terms of the Creative Commons Attribution License (http://creativecommons.org/licenses/ by/3.0), which permits unrestricted use, distribution, and reproduction in any medium, provided the original work is properly cited. (cc) BY 


\section{References}

[1] International Stroke Association. What is Stroke? Available from: https// www.Stroke.org/understand-stroke/ what-is-stroke/ [Accessed: 14 May 2019]

[2] Takashima N, Arima H, Kita Y, Fujii T, Miyamatsu N, Komiri M, et al. Incidence, management and shortterm outcome of stroke in a general population of 1.4 million Japanese-Shiga Stroke Registry. Circulation Journal. 2017;81(11):1636-1646

[3] Albright KC, Branas CC, Meyer BC, Matherne-Meyer DE, Zivin JA, Lyden PD, et al. ACCESS: Acute cerebrovascular care in emergency stroke systems. Archives of Neurology. 2010;67:1210-1218

[4] Maeshima S, Osawa A, Tanahashi N. A liaison critical pathway for stroke rehabilitation: Current status and features of western district of Saitama in Japan. Physical Medicine and RehabilitationInternational. 2015;2(1):5

[5] Steinhagen V, Grossmann A, Benecke R, Walter U. Swallowing disturbance pattern relates to brain lesion location in acute stroke patients. Stroke. 2009;40:1903-1906

[6] Martino R, Foley N, Bhogal S, Diamant N, Speechley M, Teasell R. Dysphagia after stroke: Incidence, diagnosis, and pulmonary complications. Stroke. 2005;36(12):2756-2763

[7] Logemann JA, Shanahan T, Rademaker AW, Kahrilas PJ, Lazar R, Halper A.

Oropharyngeal swallowing after stroke in the left basal ganglion/internal capsule. Dysphagia. 1993;8:230-234

[8] Maeshima S, Osawa A, Hayashi T, Tanahashi N. Factors associated with prognosis of eating and swallowing disability after stroke: A study from a community-based stroke care system. Journal of Stroke and Cerebrovascular Diseases. 2013;22:926-930
[9] Maeshima S, Osawa A, Yamane F, Ishihara S, Tanahashi N. Dysphagia following acute thalamic haemorrhage: Clinical correlates and outcomes. European Neurology. 2014;71(3-4):165-172

[10] Osawa A, Maeshima S, Matsuda H, Tanahashi N. Functional lesions in dysphagia due to acute stroke: Discordance between abnormal findings of bedside swallowing assessment and aspiration on videofluorography. Neuroradiology. 2013;55:413-421

[11] Leopold NA, Kagel MC. Swallowing, ingestion and dysphagia: A reappraisal. Archives of Physical Medicine and Rehabilitation. 1983;64:371-373

[12] Matsuo K, Palmer JB. Anatomy and physiology of feeding and swallowing normal and abnormal. Physical Medicine and Rehabilitation Clinics of North America. 2008;19(4):691-707

[13] Palmer JB. Bolus aggregation in the oropharynx does not depend on gravity. Archives of Physical Medicine and Rehabilitation. 1998;79:691-696

[14] Belafsky PC, Mouadeb DA, Rees CJ, Pryor JC, Postma GN, Allen J, et al. Validity and reliability of the Eating Assessment Tool (EAT-10). The Annals of Otology, Rhinology, and Laryngology. 2008;117:919-924

[15] Mann G. MASA, The Mann Assessment of Swallowing Ability. Philadelphia: Delmar Thompson Learning; 2002

[16] Horiguchi S, Suzuki Y. Screening tests in evaluating swallowing function. JMAJ. 2011;54(1):31-34

[17] Oguchi K, Saitoh E, Baba M, Kusudo S, Tanaka T, Onogi K. The repetitive saliva swallowing test (RSST) as a screening test of 
functional dysphagia (2). Validity of RSST. Japanese Association of Rehabilitation Medicine. 2000;37: 383-388. (Japanese)

[18] Tohara H, Saitoh E, Mays KA, Kuhlemeier K, Palmer JB. Three tests for predicting aspiration without videofluorography. Dysphagia. 2003;18:126-134

[19] Kubota T, Mishima H, Hanada M, Namba I, Kojima Y. Paralytic dysphagia in cerebrovascular disorder-Screening tests and their clinical application. Sogo Rehabilitation. 1982;10:271-278. (Japanese)

[20] Saitoh E. Comprehensive Study Report. 1999 Health Labour Sciences Research Grant of the Ministry of Welfare. Comprehensive Research on Aging and Health, 1999 research report (Central Secretariat of Aging and Health Expenses). 2000. pp. 1-18. (Japanese)

[21] Osawa A, Maeshima S, Tanahashi N. Food and liquid swallowing difficulty in stroke patients: A study based on the findings of food tests a modified water swallowing test and videofluoroscopic examination of swallowing. Japanese Association of Rehabilitation Medicine. 2012;49:838-845. (Japanese)

[22] Teramoto S, Matsuse T, Fukuchi Y, Ouchi Y. Simple two-step swallowing provocation test for elderly patients with aspiration pneumonia. Lancet. 1999;353:1243

[23] Smithard DG, O’Neill PA, Park C, Morris J. Complications and outcome after acute stroke: Does dysphagia matter? Stroke. 1994;27:1200-1204

[24] Martino R, Pron G, Diamant N. Screening for oropharyngeal dysphagia in stroke: Insufficient evidence for guidelines. Dysphagia. 2000;15:19-30

[25] Trapl M, Enderle P, Nowotny M, Teuschl Y, Matz K, Dachenhausen A, et al. Dysphagia bedside screening for acute-stroke patients: The Gugging swallowing screen. Stroke. 2007;38(11):2948-2952

[26] Osawa A, Maeshima S, Tanahashi N. Water swallowing test: Screening for aspiration in stroke patients. Cerebrovascular Diseases. 2013;35:276-281

[27] Tazawa Y, Maeshima S, Osawa A, Tanahashi N. Bedside screening test and videofluorography to detect aspiration in acute stroke patients. The Journal of Saitama Comprehensive Rehabilitation. 2010;10:48-50. (Japanese)

[28] Maeshima S, Osawa A, Miyazaki Y, Seki Y, Miura C, Tazawa Y, et al. Influence of dysphagia on short-term outcome in patients with acute stroke. American Journal of Physical Medicine \& Rehabilitation. 2011;90(4):316-320

[29] Schiele JT, Penner H, Schneider H, Quinzler R, Reich G, Wezler N, et al. Swallowing tablets and capsules increases the risk of penetration and aspiration in patients with strokeinduced dysphagia. Dysphagia. 2015;30(5):571-582

[30] Rugiu MG. Role of videofluoroscopy in evaluation of neurologic dysphagia. Acta Otorhinolaryngologica Italica. 2007;27(6):306-316

[31] Fujii N, Inamoto Y, Saitoh E, Baba M, Okada S, Yoshioka S, et al. Evaluation of swallowing using 320-detectorrow multislice CT. Part I: Single- and multiphase volume scanning for threedimensional morphological and kinematic analysis. Dysphagia. 2011;26(2):99-107

[32] Inamoto Y, Fujii N, Saitoh E, Baba M, Okada S, Katada K, et al. Evaluation of swallowing using 320-detector-row multislice CT. Part II: Kinematic analysis of laryngeal closure during normal swallowing. Dysphagia. 2011;26(3):209-217 
[33] Karnell MP, MacCracken E. A database information storage and reporting system for videofluorographic oropharyngeal motility (OPM) swallowing evaluations. American Journal of Speech-Language Pathology. 1994;3(2):54-60

[34] Cray MA, Mann GD, Groher ME. Initial psychometric assessment of a functional oral intake scale for dysphagia in stroke patients. Archives of Physical Medicine and Rehabilitation. 2005;86:1516-1520

[35] Baba M, Saitoh E. Indication of dysphagia rehabilitation. Rinsho Reha. 2000;9:857-863. (Japanese)

[36] Nishimura K, Kagaya H, Shibata S, Onogi K, Inamoto Y, Ota K, et al.

Accuracy of Dysphagia Severity Scale rating without using videoendoscopic evaluation of swallowing. Japanese Journal of Comprehensive Rehabilitation Science. 2015;6:124-128

[37] O’Neil KH, Purdy M, Falk J, Gallo L. The Dysphagia Outcome and Severity Scale. Dysphagia. 1999;14:139-145

[38] Foley N, Teasell R, Salter K, Kruger E, Martino R. Dysphagia treatment post stroke: A systemic review of randomized controlled trials. Age and Ageing. 2008;37(3):258-264

[39] Logemann JA. Dysphagia: Evaluation and treatment. Folia Phoniatrica et Logopaedica. 1995;47(3):140-164

[40] Maeshima S, Osawa A, Hayashi T, Tanahashi N. Elderly age, bilateral lesions, and severe neurological deficit are correlated with strokeassociated pneumonia. Journal of Stroke and Cerebrovascular Diseases. 2014;23(3):484-489

[41] Maeshima S, Osawa A, Yamane F, Ishihara S, Tanahashi N. Association between microbleeds observed on
T2*-weighted magnetic resonance images and dysphagia in patients with acute supratentorial cerebral hemorrhage. Journal of Stroke and Cerebrovascular Diseases. 2014;23(9):2458-2463 

Section 4

Maxillofacial and Oral

Dysphagia 



\title{
Maxillofacial and Oral Aspects of Dysphagia
}

\author{
Mohammed Basha
}

\begin{abstract}
Oral cavity/mouth is first recipient of food. Food is broken down and prepared for initial phases of digestion. The oral preparatory phase is voluntary. In this phase, food is manipulated by the tongue and teeth. A bolus which is ready to swallow is prepared. Any disruption of oral cavity functions commonly due to oral infections, space infections, facial trauma, congenital-cleft lip and palate, temporo-mandibular joint disorders, salivary gland pathology, oral cancers, radiation therapy, etc., can cause dysphagia. In this chapter, we would explain the maxillofacial and oral aspects of dysphagia along with diagnosis and treatment aspects.
\end{abstract}

Keywords: oral dysphagia, maxillofacial dysphagia, bolus, saliva, tongue, oral infections

\section{Introduction}

Facial structures and its functions are very precise and rhythmic, well-orchestrated co-ordination between various structures to achieve multiple goal at the same time. Innumerous central control of voluntary and involuntary function have been studied and documented in many forms of medical literatures. Multiple facial hard and soft tissues, nerves, muscles etc., function in a very unique and well balanced way. The extremely complex process of act of swallowing/deglutition, involves approximately 50 pairs of muscles and nerves that are responsible for preparing and transferring food and liquids from the mouth to the stomach [1]. Due to the close proximity of the pathways of swallowing and respiration, precise coordination between these functions is vital in order to avoid entry of material into the airway [2]. A problem with any of these structures can lead to dysphagia [1].

Patients with pathology in oral cavity often seek treatment for that particular sign and symptoms and there by dysphagia can be easily missed. Even though disorders of swallowing are very common and, when looked for, occur regularly in most branches of surgery. Most common cause of dysphagia is a neurological disturbance [3]. Surgeons/physicians must look for presence of a second pathology affecting their swallow and plan treatment accordingly.

\section{Anatomy of oral cavity - selected aspects}

The oral cavity, the pharynx, and the larynx are the three anatomically and functionally separate aero-digestive tract structures which form the swallowing apparatus. Normal deglutition requires precise coordination of these structures [4-7]. 
A hydrodynamic pump is created with valves by these structures that allow food and liquid to be transferred into the stomach without aspiration $[4,6]$. Swallowing comprise of three phases, based on the location of the bolus in the swallowing framework. Oral phase is voluntary and it set off involuntary pharyngeal and esophageal phases [4-6]. Large number of diseases causes symptoms of dysphagia affecting the quality of life of patients [6]. Understanding of the anatomy and physiology of deglutition is required to treat dysphagia.

The oral cavity inlet is guarded by the upper and lower lips. The other units of the mouth are cheeks, maxillary and mandibular teeth, gums and periodontium and periodontal ligaments, hard and soft palate, tongue and floor of mouth. The oral phase of swallowing occurs in the oral region. Masticatory performance is defined as the percentage of particle size distribution of food when chewed for a given number of strokes [8]. This evaluates the quality of chewing. Masticatory performance reflects the capacity to reduce the size of food particles and the number of chews necessary to render food ready for swallowing $[9,10]$. Quality of mastication performance is affected by multiple factors like number of teeth in functional occlusion [11-13], biting force [14], dentures [15], Implants and or artificial prosthesis and salivary flow rate - which declines (masticatory performance) with a reduction of salivary secretion [16]. Bolus preparation happens between upper arch (maxillary arch) and lower arch (mandibular arch). Oral cavity transforms into oropharynx posteriorly.

Upper and lower Lips form a sphincter using the orbicularis oris muscle. Intraorally the lips have labial mucosa (mucous membrane), which extends laterally on the inner surface of bilateral cheeks as buccal mucosa. Cheeks contain the buccinator muscles and the buccal fat pads. Palate anteriorly is hard palate formed by fusion the palatine process of both maxillae anteriorly and horizontal plates of palatine bone fused at transverse palatal suture. The soft palate is located posteriorly. Anteriorly, it is continuous with the hard palate and with palatine aponeurosis. The posterior border of the soft palate is free, and has a central process that hangs from midline known as uvula. The soft palate is mobile, and comprised of muscle fibers covered by mucous membrane. The soft palate continues laterally with palatoglossal and palatopharyngeal folds, which joins the tongue and pharynx respectively [17].

\section{Oral clinical conditions for dysphagia and treatment aspects}

Patients with pathology in oral cavity often seek treatment for that particular sign and symptoms. Dysphagia can be easily missed. These conditions can be congenital or acquired.

Congenital conditions which are most commonly encountered in our practice are facial clefts; cleft lip and palate. There are always lots of emotions and expectations of parents during treatment. Feeding in the cleft lip and palate setting is the main challenge for the parents, pediatricians, surgeons and nurses. In a study, 97\% of parents of cleft infants thought to discuss feeding challenges, $95 \%$ thought it was important to have a demonstration of feeding [18]. Poor oral suction, inadequate volume intake, lengthy feeding time, nasal regurgitation, excessive air intake, and coughing or choking are the feeding challenges [10]. The anatomic-structural deformities of cleft infants usually affect the oral phase of the suck-swallow-breathing mechanism. Cleft with syndrome, the risk of poor feeding is 15 times more [19].

The importance of adequate nutrition in cleft patients care is indispensable especially during presurgical and postsurgical period. In cleft palate patients, the ability to secure adequate seal and suction is remarkably reduced [20]. Poor intake abilities and diminished sucking efficiency causes the cleft baby with reduced oral intake, reduced weight gain and inadequate growth [21] (Figure 1). 


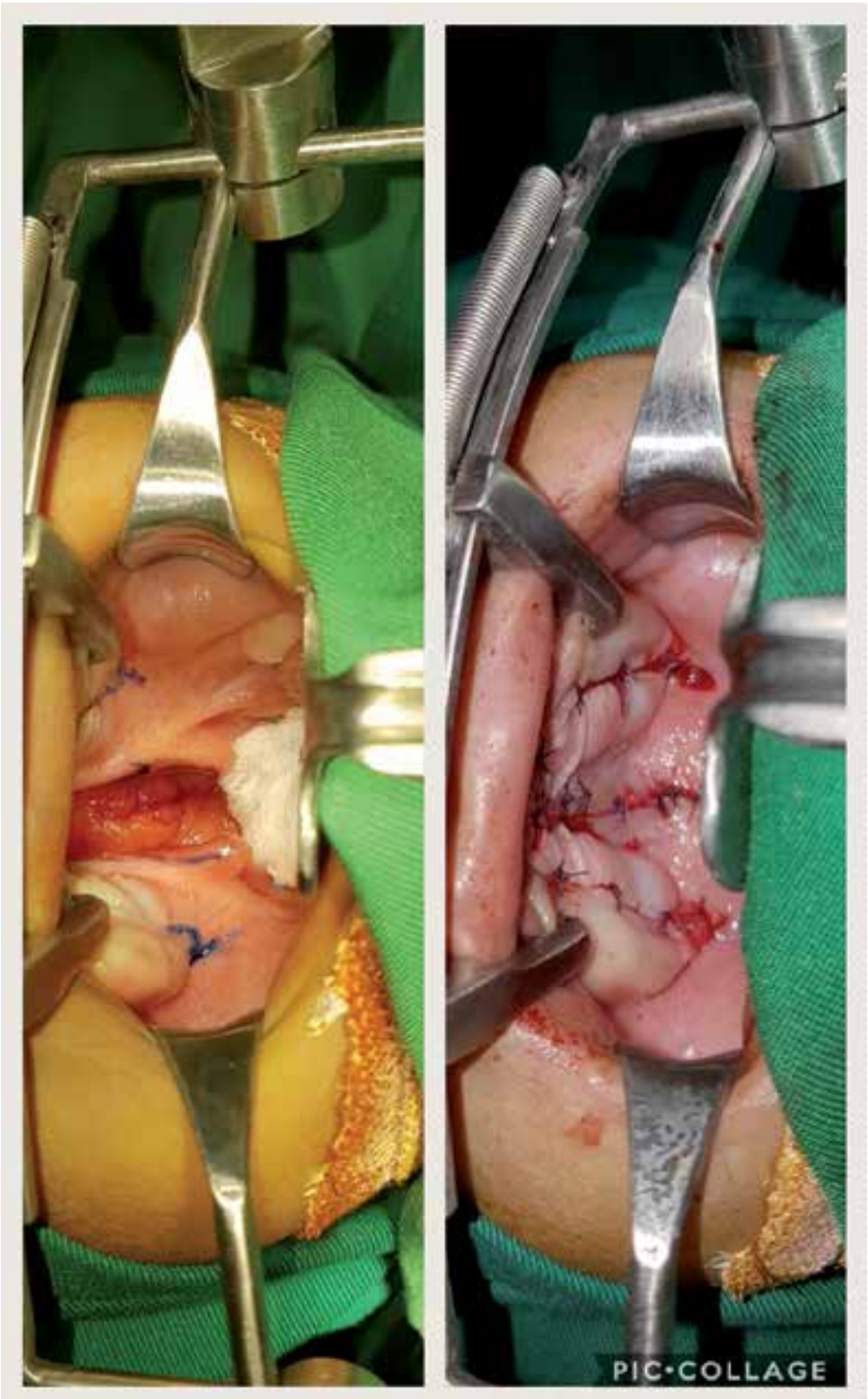

Figure 1.

Cleft of the palate pre-and postsurgery.

Bottles commonly used for feeding infants with cleft deformities include the Cleft Palate Nurser, Mead Johnson LLC, Glenview, IL; Medela Special Needs Feeder, Pigeon Bottle and vented bottles such as the Dr. Brown Bottle [22].

Post-surgery the parent face fresh challenges for feeding. Feeding protocols during post-surgery phase is contentious subject among surgeons. Surgeons recommend 3-5 days of bottle holiday, they recommend syringe feeding. Various studies bespeak that the mechanical effect of feeding have little effect on wound complications or incision breakdown [23-26].

A research conducted by $\mathrm{Al} \mathrm{Hadadi}$ and colleagues showed that aspiration pneumonia was diagnosed in $15.8 \%$ of all Cleft palate babies, which was analogous to a study from Malaysia whose results were 14\% [27, 28]. The reason for high incidence 
of aspiration pneumonia in orofacial cleft is malfunctioning of the palatal muscles and cleft of the palate leading to difficult swallowing and aspiration. It is also been reported that the hyoid bone is abnormal in cleft patients [29].

\section{Acquired causes for oral dysphagia}

Oral health is defined as "a state of being free from chronic mouth and facial pain, oral and throat cancer, oral infection and sores, periodontal(gum) disease, tooth decay, tooth loss, and other diseases and disorders that limit an individual's capacity in biting, chewing, smiling, speaking, and psychosocial wellbeing" [30]. According to the World Health Organization dental caries and periodontal diseases have high prevalent rate of nearly 100 and 90\% respectively. Aspiration pneumonia in the medically compromised and elderly, and post-operative infections can be prevented by proper oral health and hygiene [31-33].

Loss of tooth will disturb the pattern of pre- swallow and swallowing behaviors. Especially with older individual with loss of multiple teeth find it onerous in chewing and swallowing [34]. Chewing ability directly depends on remaining functional teeth with functional occlusion [11-13].

Oral preparatory stage which is affected the most due to loss of teeth, as loss of tooth diminish chewing ability and causes difficulties in forming the bolus. Bolus size was reported to increase with an increasing number of missing teeth, with larger boluses potentially interfering with optimal swallowing [35]. An ill formed bolus may be difficult to transport smoothly and efficiently into the pharynx, thus leading to additional swallowing abnormalities.

Replacement of loss of teeth by prosthetic teeth/dentures is an effective way to increase the functional masticatory unit. Prosthetic rehabilitation to maintain appropriate mandible position with good occlusion is important in smooth swallowing in older individuals [36].

A study in which relationship between the number of teeth in function, denture use and subjective swallowing problems in a sample size of 5643 individuals aged 40-89 years concluded that individuals with fewer functional teeth or no denture use were likely to have subjective swallowing problems, and suggested that, even if people have few teeth, denture wearing contributes to prevention of swallowing problems [34].

An extensive study conducted in 289 elderly people aged 60 years and older, to evaluate the relationship between the number of teeth, denture wearing, and swallowing by a noninvasive cervical auscultation technique. They concluded that $31.1 \%$ had swallowing impairment. About $20 \%$ of patients had swallowing impairment in subjects with 20 or more teeth with denture whereas subjects with 20 or more teeth without dentures had swallowing impairment by $26.5 \%$. Swallowing impairment was found in $38.9 \%$ in less than 19 teeth without prosthesis. About $57.7 \%$ had swallowing impairment with less than nine teeth without dentures. And, $66.7 \%$ completely edentulous patients without dentures had difficulty in swallowing, whereas it was to be $28.7 \%$ with complete denture prosthesis [37].

Researcher have found that there was higher frequency of entry of secretions, food/liquid, or any foreign material into the laryngeal vestibule above the level of the true vocal folds (laryngeal penetration) in edentulous elderly people who are not wearing any denture prosthesis, but laryngeal penetration was reduced in edentulous elderly people when wearing dentures [38].

The precision fit of dentures has positive effect on the swallowing function. Wearing ill-fitting dentures, particularly in the case of a complete denture, may interfere with achieving sufficient occlusal contact. Insufficient occlusal contact 
reduces masticatory performance and causes impairment of swallowing activity. In a study which shows ill-fitting dentures increases duration of swallowing in elderly patients using electromyography. Normal swallowing duration form 12 to 70 years age person ranges between 0.80 and $1.60 \mathrm{~s}$. They claimed that in ill-fitting poor prosthesis the swallowing time was $1.84 \mathrm{~s}$ and the time was reduced to $1.28 \mathrm{~s}$ in wellfitting prosthesis. The quality and fit of prosthesis plays a vital role to improve swallowing ability in elderly individuals [39]. By repairing or relining ill-fitting dentures showed the pharyngeal transit time was shorter when compared to not wearers. Increase of pharyngeal transit time increases the risk of aspiration [40]. A person who has inadequate natural masticatory teeth and are without wearing dentures had a 91\% greater risk of dementia than those with adequate natural mastication [41].

Dry mouth or xerostomia is a condition which results in dryness of mouth due to decrease in saliva secretion. It is common in elderly population and has an incidence of $20-60 \%$ [42]. Xerostomia affects the oral preparatory and oral phases of swallowing, causes impaired bolus formation and transport [43]. Swallowing disorders in xerostomia can be managed with oral moisturizers, lubricants, and careful use of fluids during eating [44]. It is considered that tooth brushing helps to improve oral hygiene and increased salivary flow rate. Mechanical stimulation of the salivary glands during tooth brushing can promote the discharge of saliva [45]. Tooth brushing may activate impulses to both major and minor salivary tissues following stimulation of oral and pharyngeal regions, causing salivation [46]. In a study by Papas and colleagues showed that tooth brushing increased salivary flow in people with medication-induced dry mouth [46]. Another study stated that elderly people with tooth brushing frequency less than twice per day were more likely to have dry mouth [45].

Hyper salivation may produce swallowing problems caused by aspiration of saliva. It's induced by inflammations of oral cavity, Parkinson's disease, epilepsy, or medicine such as pilocarpine and cholinesterase. Neurological disorders cause excessive pooling of saliva in oral cavity, with unintentional loss of saliva from mouth - drooling of saliva [47]. Drooling impair masticatory function and can cause aspiration.

Infections from the teeth are referred as odontogenic infections; these are very common in dental and maxillofacial practice. These infections can be simple/localized or can become very aggressive involving spaces of neck and deep neck region [48]. Flynn showed that fever, swelling, dysphagia and trismus (Figure 2) were the symptoms most commonly observed in patients hospitalized for odontogenic infection [49].

Deep neck space infections can spread along the facial spaces of the head and neck, inducing life-threatening deep space infection associated with a high risk of complications (upper airway obstruction, mediastinitis, thoracic empyema, pericarditis, septic shock) [50-53] (Figure 3). Neck spaces communicate with one another, results in spread of infection over larger area. Neck spaces consist of loose connective tissue between the deep cervical fascia (superficial, middle and deep) [54].

Ludwig's angina is an aggressive infection of facial and deep neck spaces, often of dental origin, characterized by a rapid spread of cellulitis in the submandibular and sublingual spaces [55] (Figure 4). It was described by Karl Friedrich Wilhelm von Ludwig in 1836 [56]. The origin of Ludwig's angina is odontogenic in $90 \%$ of cases, males are more commonly affected than females, and associated teeth are usually mandible molars- second and third molars [57-59]. The dental roots of these teeth usually lie below the mylohyoid ridge, infection from these teeth spread directly into the submaxillary spaces. With all the spaces which are well connected there is continuous spread of infection in sublingual space, pharyngeal and retropharyngeal spaces of neck [60]. 
Voice and Swallowing Disorders

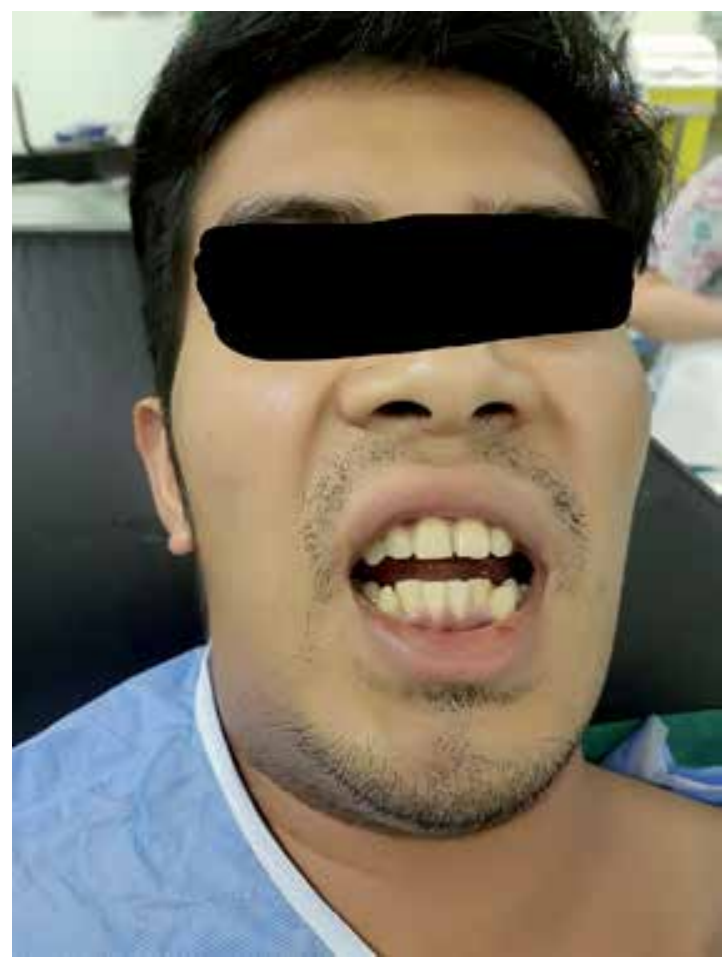

Figure 2.

Severe trismus in oral and neck infection.

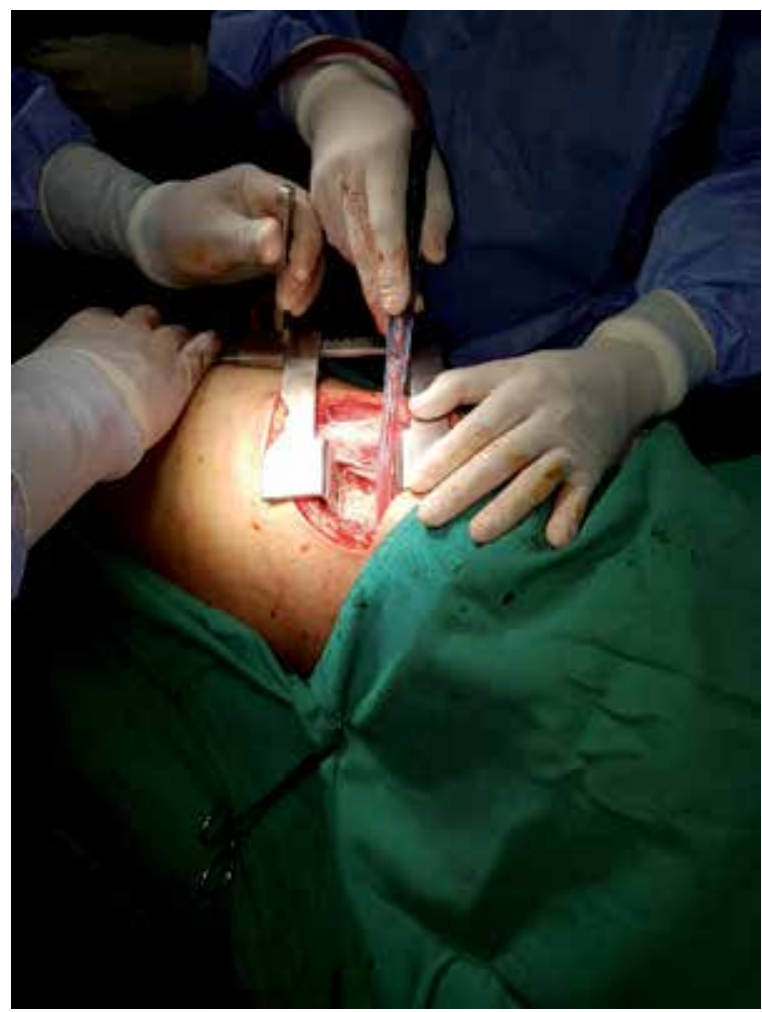

Figure 3.

Thoracotomy for descending oral infection and mediastinitis and empyema. 


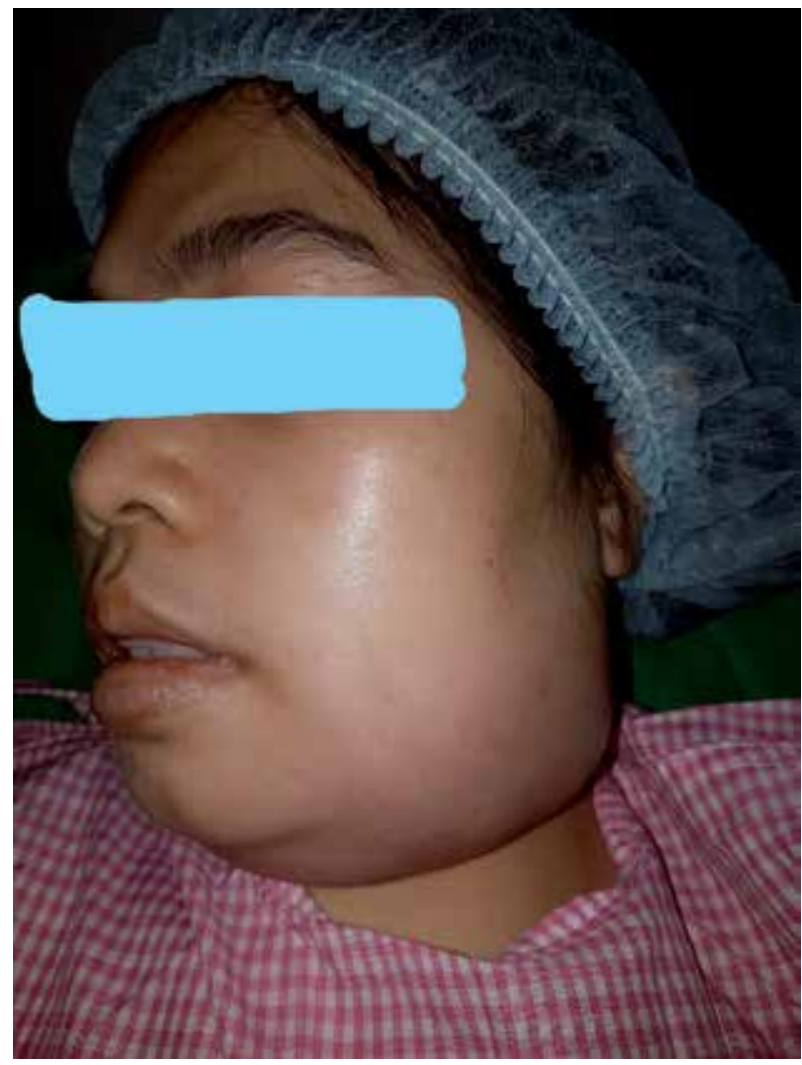

Figure 4.

A case of submandibular and neck space infection.

Patients with active or chronic oral/dental infections, poor oral care, medically compromised conditions like uncontrolled diabetes mellitus, malnutrition, drug abuser, AIDS, immunosuppression are highly susceptible for developing space infections. Ludwig's in Children can occur without any predisposing condition [60].

Clinical features have bilateral suprahyoid swelling, hard indurated cardboardlike consistency. It is non-fluctuating and tender on palpation. The mouth is open and the tongue is raised and is in contact with the palate. Patient has dysphagia, drooling of saliva, and breathing/airway obstruction, which are the most salient presenting. Signs and symptoms of the illness depend on the spaces involved, and include pain, fever, malaise, fatigue, swelling, odynophagia, dysphagia, trismus, dysphonia, otalgia, and dyspnea which are due to cellulitis, aided by the awkwardness resulting from the position of the tongue [61]. Redness is usually seen on skin in area front of the neck (Figure 5). The diagnosis in patients with Ludwig's angina is based on clinical findings. Panoramic radiography can help to discover the origin of the dental infection, while a cervico-thoracic CT scan with contrast is diagnostic (Figure 6). Ludwig's angina is potentially fatal, and its mortality rate can reach up to $50 \%$ [60] if not treated timely. Streptococci, Peptostreptococcus species, Staphylococcus aureus, and anaerobes are the most commonly cultured organisms from space infections [61]. The odontogenic infections that may cause Ludwig's angina can largely be prevented by timely interventions and periodic dental care.

Ludwig's angina can cause life threatening complications of airway obstruction, which is an acute emergency. Descend of infection into deeper plane can cause mediastinitis, pleural empyema, thrombosis of jugular vein, pericarditis, generalize sepsis and bacteremia, hematogenous dissemination to distant organs, DIC $[62,63]$. 
Voice and Swallowing Disorders

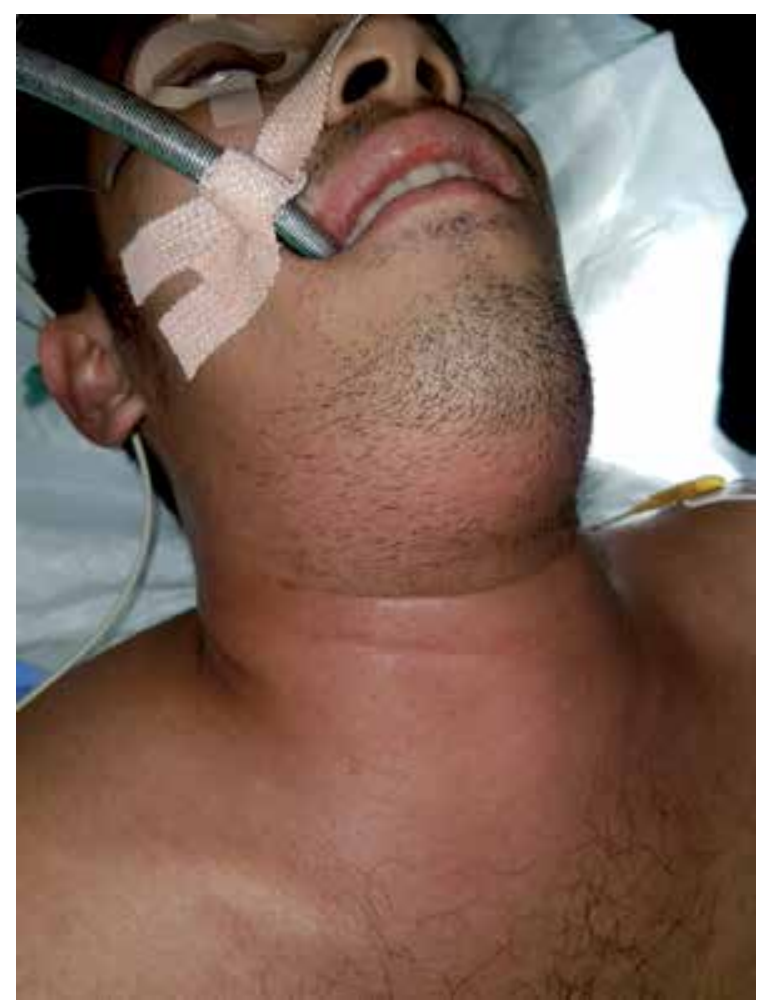

Figure 5.

Deep neck infection with cellulitis.

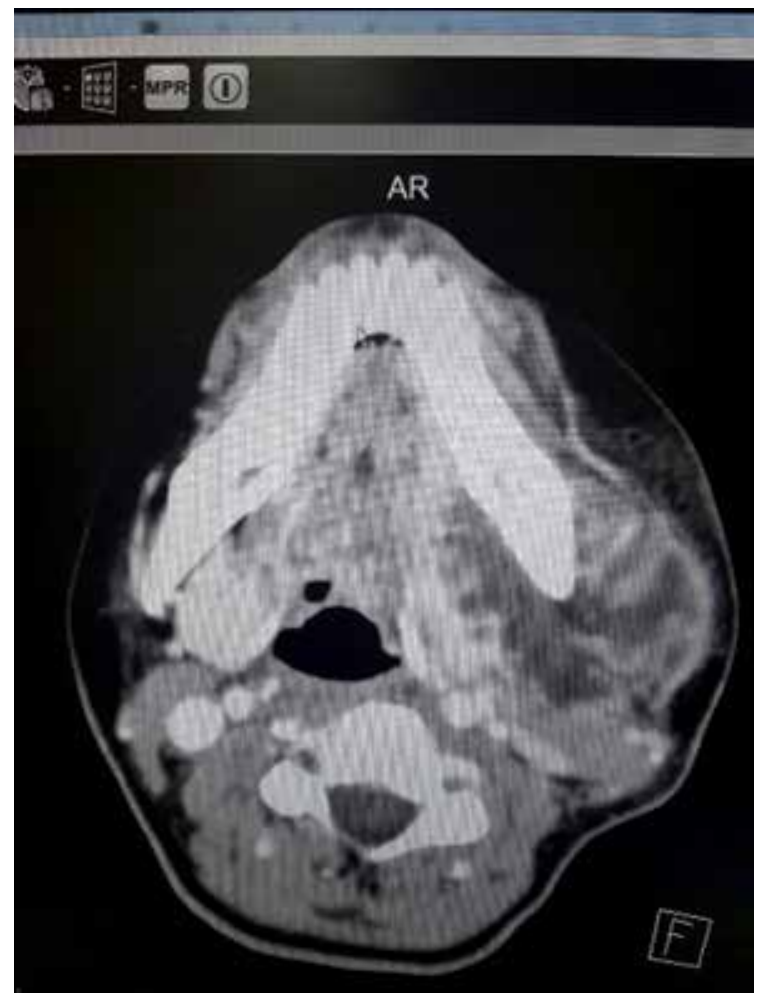

Figure 6.

CT showing neck abscess with tracheal deviation to opposite side. 
Antibiotics, airway management, surgical drainage remains the key in its management. In life saving conditions, airway protection, emergency surgical drainage of the abscess followed by antibiotics, proper diagnosis and its management is the key to managing deep neck space infections (Figure 7).

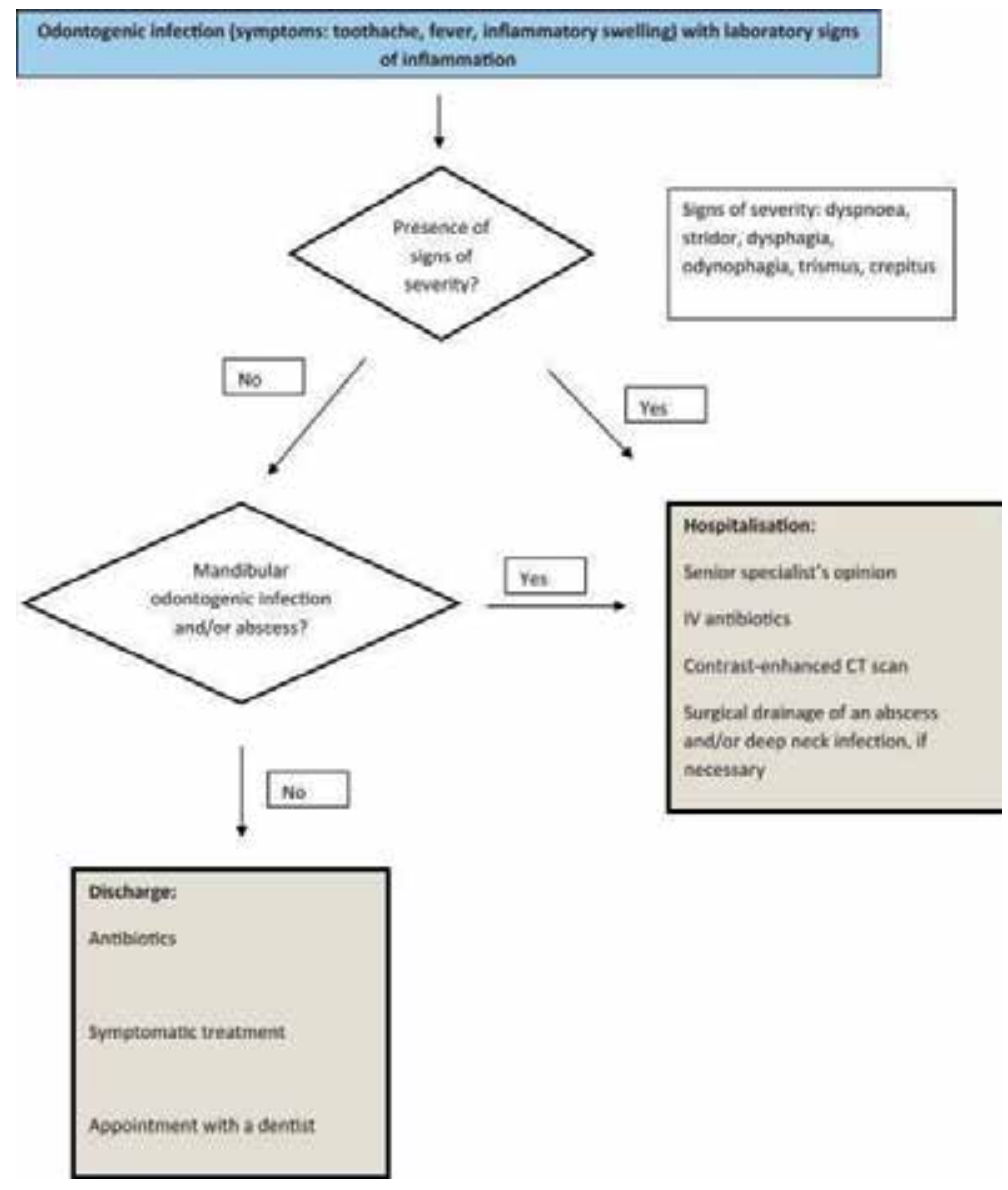

Flow chart for patients with odontogenic infection attending the emergency department- taken from the work of AlOtaibi and companions [48].

Acute necrotizing ulcerative gingivitis (ANUG), or Vincent's infection, or trench mouth is a disease affecting the oral cavity which was noted in the Greek army by Xenophon. Soldiers suffered sore, ulcerated, and foul-smelling mouths. Similar occurrences were noted among soldiers in World War I who suffered severely from this condition. The disease is commonly found in young middle-aged, which is characterized by painful, hyperemic gingiva with sharply punched-out interdental papillae, gingival bleeding on gentle probing, the presence of a gray necrotic pseudo membrane, and a fetid mouth odor. Increased dysphagia, severe pain, hyper salivation, facial pain and swelling, accompanied by chills and fever. Conservative and supportive treatment measures based on good oral hygiene and aimed at eliminating causative factors usually result in marked improvement of the patient's condition within a relatively short period of time [64].

Viral Infections and its oral manifestations like Herpes simplex infections cause numerous shallow, irregular, necrotic ulcers, with surrounding edema and inflammation, widespread over the inside of both upper and lower lips, on the dorsal and ventral surfaces of the tongue, cheeks and soft and hard palate. Usually, the ulcers are extremely painful to touch. Patient complains of severe pain on eating, chewing and swallowing. 
Recurrent aphthous stomatitis (RAS) can be defined as a recurrent oral necrotizing ulceration, the etiology of which remains obscure. Mikulicz and Kummell first described the chronic recurrent nature of this disease. In 1911, Sutton described a more severe form of RAS as periadenitis mucosa necrotica recurrens. At present, the disorder is more simply referred to as mild and severe aphthous or minor and major recurrent aphthous ulcers. In 1937, Behcet described a mucocutaneous-ocular syndrome that now bears his name and is characterized by a triad of symptomsrecurrent oral ulcerations, recurrent genital ulcerations, and ocular inflammation. All these diseases cause oral ulcerations, where in the patients modify their oral intake just to avoid pain. If ulcers on posterior tongue, oropharynx regions, these
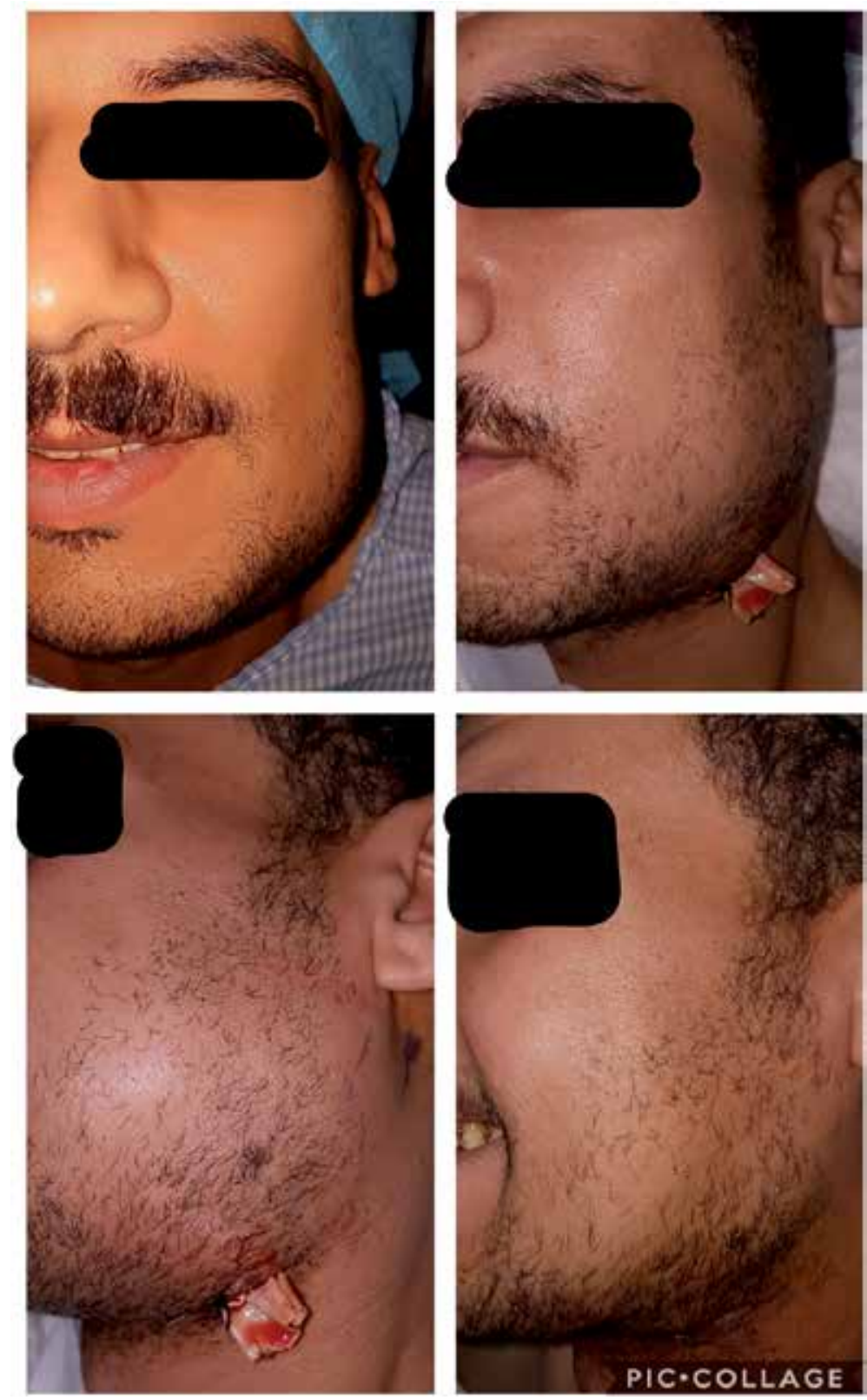

Figure 7.

Neck infection: pre surgery, with drains, post treatment. 
cause severe dysphagia (Figure 8). Treatment options include tropical steroids oral pastes, steroids, Vitamin C, immune suppressants based on clinical features, severity and clinicians treatment protocol [65].

Salivary gland pathology also contributes for dysphagia. Most common salivary gland pathology causing symptoms of dysphagia encountered in practice are ranula which occurs in the floor of mouth. Ranula is an extravasation cyst and may develop from extravasation of mucus after trauma to the sublingual gland (Figure 9) and rarely submandibular gland or obstruction of salivary ducts [66]. The lesion forms due to extravasation of mucus and subsequent formation of a pseudocyst. It is characteristically large $(>2 \mathrm{~cm})$ and most reported ranulas are $4-10 \mathrm{~cm}$ in size and rarely bigger than $10 \mathrm{~cm}$ [67]. When ranula becomes larger, it acquires a blue color

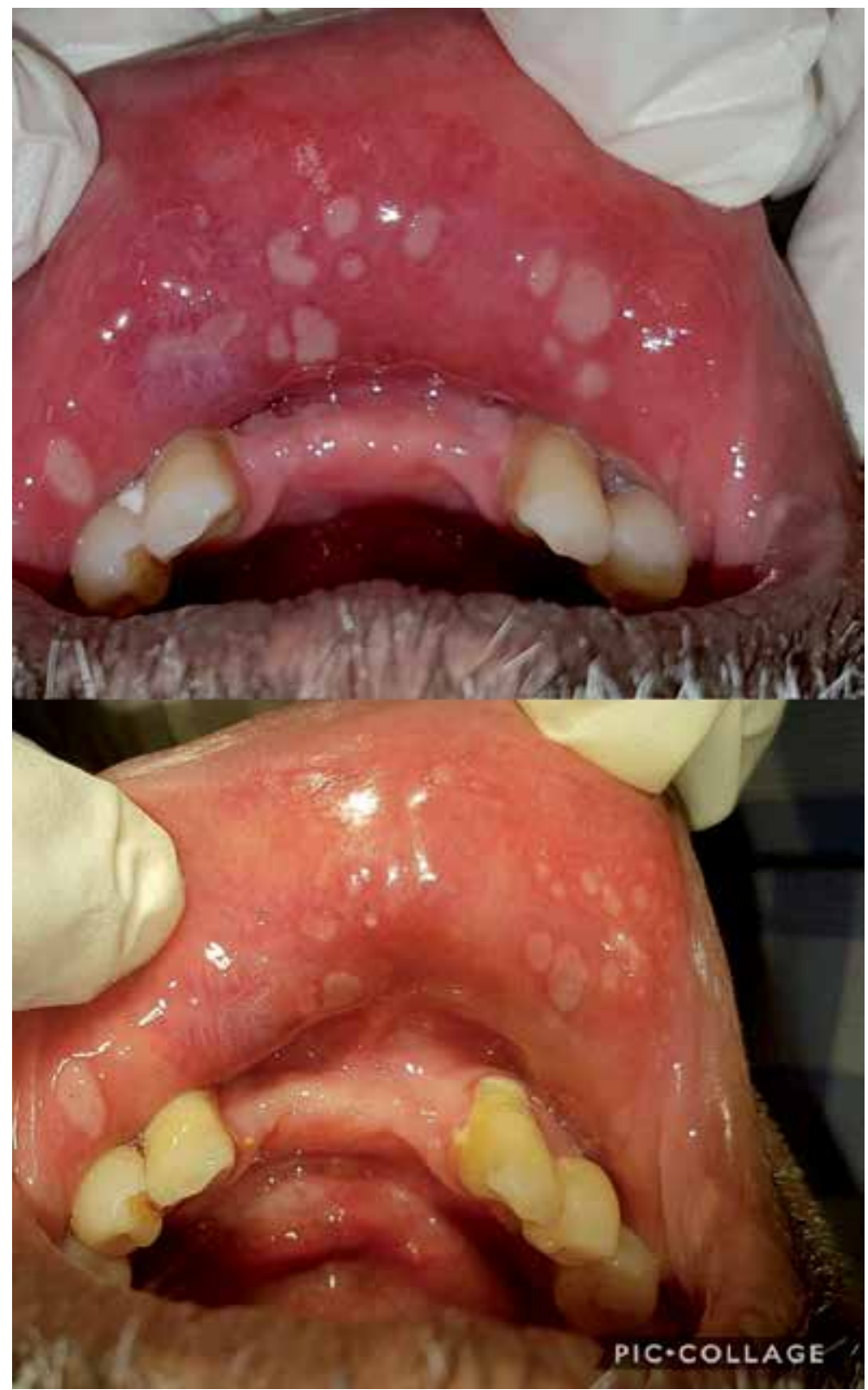

Figure 8.

Oral ulcers: pre and post treatment. 


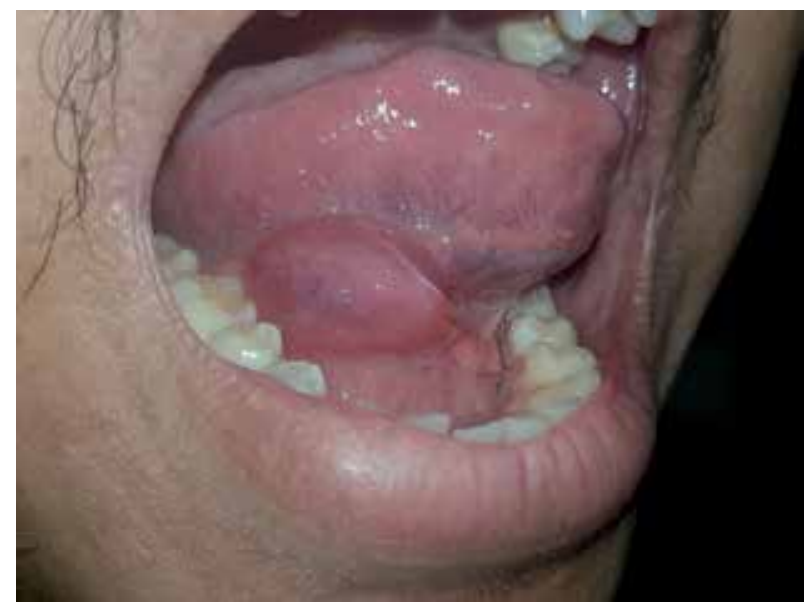

Figure 9.

In oral ranula.

and resemble frog's belly. Big sized ranulas may cause deviation of the tongue with associated difficulties in speech, swallowing and mastication [68]. It commonly occurs in the second and third decades of life and the reported male to female ratio is $1: 1.3$. The most common factor is that trauma causes direct damage to the duct of the sublingual gland [69]. Treatment option most commonly used is surgical removal of the lesion along with the involved salivary gland.

Bleeding in floor of mouth following trauma causes rapid elevation/enlargement of the tongue secondary to sublingual hematoma can cause life-threatening airway obstruction, necessitating prompt recognition and management of this condition [70]. Etiology of traumatic lingual hematomas include: motor vehicle accidents associated with mandible fractures, assault, child abuse, and seizures. Spontaneous lingual hematomas are usually a result of an inherited coagulopathy or patients on anticoagulant treatment [71-74]. Clinical signs of sublingual or lingual hematoma may include mass like swelling, hematoma, dyspnea, stridor, dysphagia and dysphonia. Tongue enlargement causing upper airway obstruction as it is displaced posterior-superiorly. Securing the airway is the first most important step in the management of these patients. Once airway is secured, hemostasis is focused at based on the primary cause. Treatment options include observation, airway control, steroids, antibiotics, reversal of any coagulopathy, embolization and surgical intervention. Surgical intervention becomes necessary in instances where embolization is unavailable or unsuccessful. This most commonly entails extra-oral ligation of the lingual artery and which requires a detailed understanding of the involved anatomy. Being able to recognize the initial presentation, underlying cause and relevant anatomy of lingual hematomas is critical to the proper management and treatment of this condition [75].

Sublingual hematoma caused by Placement of dental implants in the anterior mandible has been reported. Dental implants placement is a routine procedure done in outpatient departments of dental clinics. Endosseous dental implants placed in canine-premolar region have caused severe bleeding in the loose tissues of the floor of the mouth, the sublingual area and the space between the lingual muscles. Lingual surface of mandible is very vascular. There is rich anastomoses sublingual branch of the lingual artery which anastomoses with the submental artery, a branch of the facial artery, and the incisive arteries, branches of the inferior alveolar artery. Plexus lies very close to the interforaminal lingual cortical plate of the mandible and severe hemorrhage from this region has been reported as a complication of implant 
placement and other surgical procedures. Many unnamed accessory foramina on the lingual surface of mandible have been reported after anatomical studies by Hofschneider et al., 1999, McDonnel et al., 1994, Loukas et al., 2008. Formation of a hematoma in the floor of the mouth cause tongue getting pushed up, difficulty in swallowing, dysphagia and acute airway obstruction which may require emergent airway protection via intubation or tracheostomy. Accurate preoperative assessment mandible lingual surface with the help of dental CT/cone beam CT and accurate determination of implant length, avoiding lingual perforation, close post-operative follow up is recommended to prevent this complication [76].

Severe Maxillofacial (Figure 10), neck injuries and dysphagia are wellestablished complication especially in polytrauma admitted to intensive care unit. Incidence of maxillofacial injuries in polytraumatised patients is relatively high (25.4\%) [77]. Approximately $35 \%$ are primarily affected by complex midfacial fractures. Every 13th polytrauma patient $(7.7 \%)$ presents a spinal cord injury with neurologic deficit [78]. The risk for pulmonary aspiration is very high in these

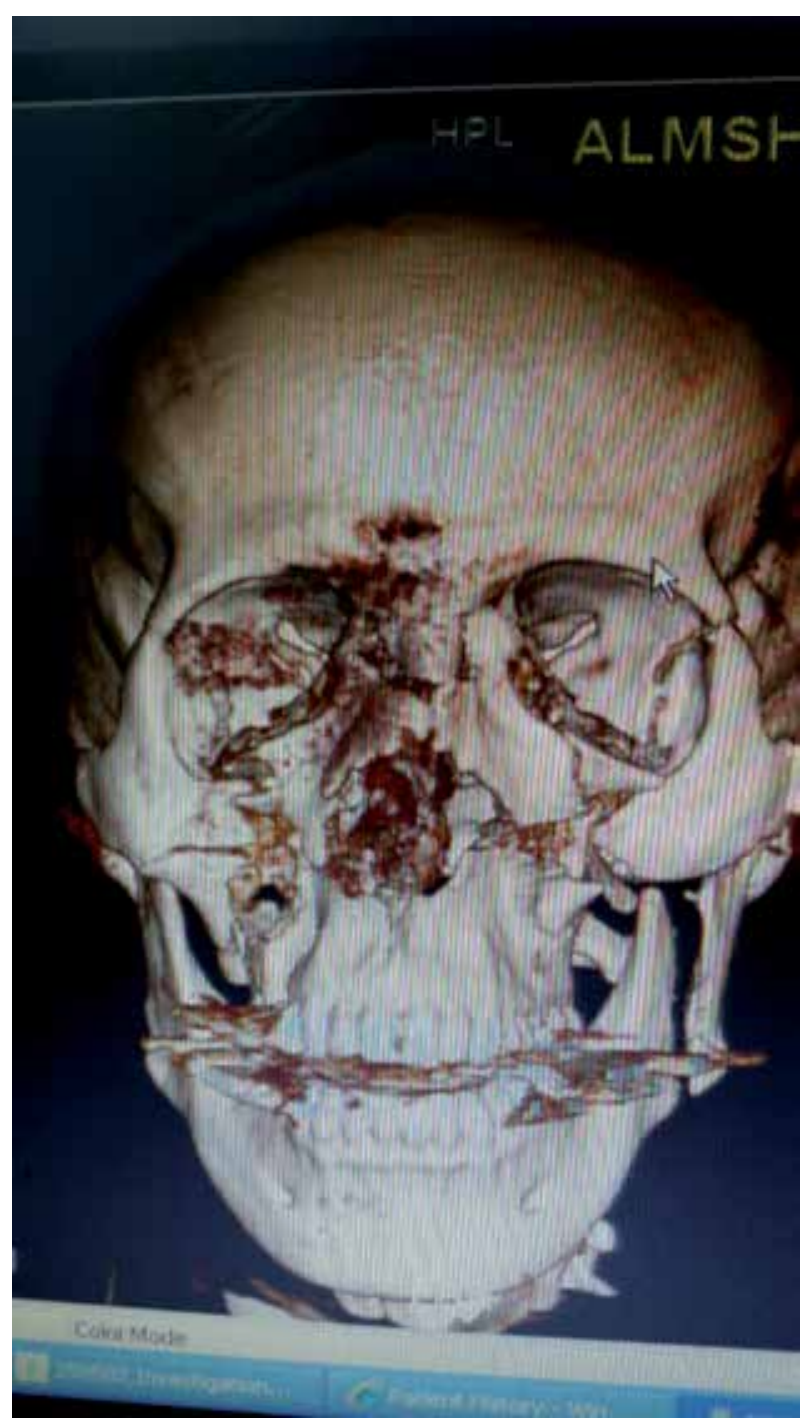

Figure 10.

Pan facial fracture. 
patients, which is a leading cause of pneumonia [79], prolong hospitalization and increase mortality rates. High-energy trauma may result in some uncommon types of mandibular fracture, which cause the bony framework to destabilize (flail mandible) (Figure 11), and lead to partial or complete occlusion of the oropharyngeal inlet. Bilateral mandible fracture especially involving the parasymphyseal region causing airway obstruction and glossoptosis is known as flail mandible fracture. The mandibular deformity caused by bilateral parasymphyseal fracture usually displacing the fractured mandible posteriorly making the "Andy Gump" face appearance; a face with deficient chin. Airway obstruction can also occur due to loose teeth dislodgement as a result of impact/trauma, foreign bodies such as oral prosthesis, hematomas of the sublingual and nasopharyngeal region [80]. Fractures of parasymphyseal region cause lack of bony continuity which further causes collapse of the genioglossus and intrinsic tongue muscles into the oropharynx. Reestablishment of a patient airway is most important aspect. Emergency reduction of the fracture fragments and temporary stabilization of fracture fragments with the help of bridal wire, anterior traction of tongue with sutures to relieve airway, using oropharyngeal or nasopharyngeal airway, or by endotracheal tube according to the condition and situation of patient. Tracheotomy can always come handy for emergencies in which airway establishment is difficult.

Zachariades reported a rare case of Laryngeal Incompetence Following Facial Trauma. Patient was diagnosed having Le Fort I type of fracture, bilateral fractures of the mandibular condyles, and a comminuted fracture of the symphysis. Patient experienced respiratory difficulty that was alleviated when the mandible was pulled forward. Tracheostomy was done, followed by maxillomandibular fixation. Post

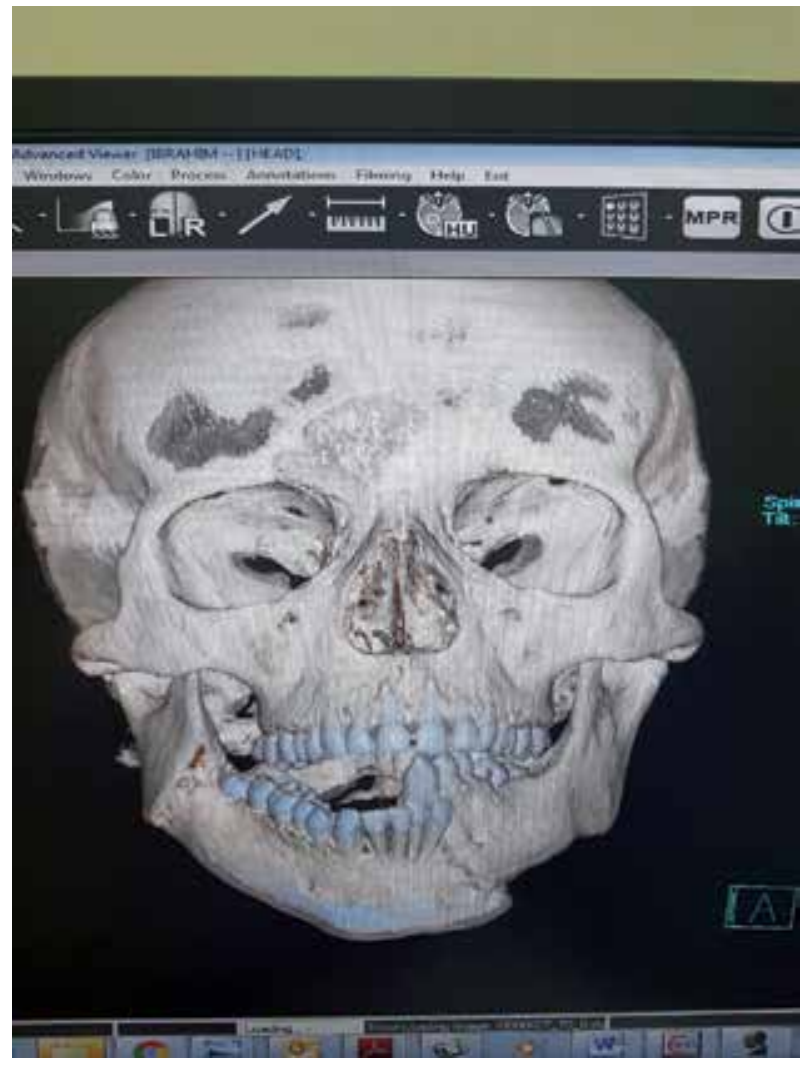

Figure 11.

Flail unstable mandible fracture. 
operatively nasogastric feeding for 2 days. When the patient was given liquids orally, he would cough; leak was noted around the tracheostomy site. Initial diagnosis of perforation of the esophagus and esophageal-tracheal communication was suspected.

Barium swallow were performed and there was no esophageal-tracheal communication. They diagnosed the condition as laryngeal incompetence. Patient was subsequently sustained on intravenous fluids and Nasogastric feeding. The tracheostomy was kept open throughout the entire period of fixation. When fixation was released after 22 days, they found that laryngeal incompetence had almost completely vanished. The author discusses the reasons for laryngeal incompetence were tracheostomy, general anesthesia administration through tracheostomy, endotracheal cuff, overextension of head during reduction and wiring fractures [81].

A detail prospective evaluation study was conducted by Tiwary and companions on maxilla-facial trauma and associated Styloid process fractures, they found that in 84 patients with road traffic accident causing maxillofacial trauma, 27 patients had styloid process fracture. In maxillofacial fractures: mandible and multiple facial fractures were associated with styloid process deformity. Their results were Styloid process fracture with mandibular fracture was found in 52\%. Clinically patients have dysphagia, dull ache in the throat, trismus, foreign body sensation, restricted lateral mandible movement, pain on turning the head to one side, otalgia, tinnitus, and tenderness to palpation of the tonsillar fossa and retromandibular regions. Management includes soft diet, analgesics and muscle relaxants as conservative therapy. Surgical excision of the distal portion of the fractured process is indicated when the patient's symptoms do not subside within a reasonable period of time [82].

Tumors located in the mouth and oropharynx are associated with dysphagia and poor swallowing function, exerting a negative impact on the quality of life of patients who have undergone resective/reconstructive surgery with or without radiotherapy for the treatment of head and neck cancer [83].

Cancer of the lips, oral cavity, oropharynx, hypopharynx, larynx, and esophagus is the fifth most common form of cancer in the world [84, 85].

Dysphagia is a common and debilitating complication of head and neck cancers (HNC), and its treatment places substantial physical and psychosocial burdens on patients [86].

Dysphagia in HNC cripples the quality of life and It can also cause life-threatening complications like aspiration pneumonia [87].

Klingelhöffe and companions in there research have stated that $98 \%$ of their patients had a swallowing impairment, and in that $10.2 \%$ patients who were unable to swallow. The size of tumor, nodal stage, reconstruction by flap and poor dental status strongly contribute to swallowing impairment. There was an increase in swallowing difficulty in patient with $\mathrm{T} 2$ than $\mathrm{T} 1$, closure with flap reconstruction over primary closure patients [87].

Chen and companions in their research have claimed that, their patients had difficulty in swallowing dry foods, hard food, and dysphagia was interfering with enjoyment or Quality Of Life (QOL). Patients with tumors of the tongue and buccal cancer had worse functional dysphagia QOL. Buccal cancer also has worse overall dysphagia QOL, functional dysphagia QOL, and physical dysphagia QOL. Emotional dysphagia QOL was associated with poor swallowing ability and depression [88].

Cancers of the tongue are associated with swallowing difficulty in all the phases of swallowing. In a study by Huang and colleagues, they found that there was restriction in tongue movement, with a delay in oral transit time, decreased hyoid bone elevation, aspiration was noted in many, vallecula epiglottica, and residual material in the pyriform sinuses were observed post-surgery. Patient with tongue cancer were associated to have higher incidence of silent aspirations, with 
no symptoms, this is dangerous condition that may cause pneumonia and even loss of life [89].

Dysphagia treatment or management for postoperative cancer patients is multistep and complex in nature. There is involvement of Stomatology surgeons, radiation and medical oncologists, dieticians, speech pathologists, and psychologists as a team to address the issues as soon as it is suspected, identified and should be addressed [90].

\section{Conclusion}

Oro-pharyngeal dysphagia is always undiagnosed and underestimated as the physician/surgeon focus on the primary disease. Impact of dysphagia should not be underestimated by the surgeon. Swallowing ability and depression are the most important factors associated with dysphagia-specific health-related quality of life. Swallowing rehabilitation programs are recommended to help cope with swallowing rehabilitation and dysphagia. Oral care is of prime importance, we suggest it's important to maintain good oral habits to achieve good systemic health, as mouth is the mirror for good health. Identification of patients who are more likely to have dysphagia, an interdisciplinary preventive interventions during treatment, long-term preventive/curative interventions and aim of minimizing the negative impacts of dysphagia on the nutritional status and quality of life of patients.

\section{Acknowledgements}

I thank to all my teachers, family and friends.

\section{Conflict of interest}

No conflicts of interest.

\section{Author details}

Mohammed Basha

Department of Dentistry/OMS, Aster Sanad Hospital, Riyadh, Saudi Arabia

*Address all correspondence to: dr.salmanbasha@gmail.com

IntechOpen

(C) 2019 The Author(s). Licensee IntechOpen. This chapter is distributed under the terms of the Creative Commons Attribution License (http://creativecommons.org/licenses/ by/3.0), which permits unrestricted use, distribution, and reproduction in any medium, provided the original work is properly cited. (cc) BY 


\section{References}

[1] Hennessy M, Goldenberg D.

Surgical anatomy and physiology of swallowing. Operative Techniques in Otolaryngology-Head and Neck Surgery. 2016;27(2):60-66. DOI: 10.1016/j. otot.2016.04.002

[2] Walton J, Silva P. Physiology of swallowing. Surgery (Oxford). 2018;36(10):529-534. DOI: 10.1016/j. mpsur.2018.08.010

[3] Counter PR, Ong JH. Disorders of swallowing. Surgery (Oxford). 2018;36(10):535-542. DOI: 10.1016/j. mpsur.2018.08.004

[4] Shaker R, Belafsky PC, Postma GN, et al. Miller Overview of Deglutition and Swallowing, Principles of Deglutition: A Multidisciplinary Text For Swallowing and its Disorders.

New York: Springer Science \& Business Media; 2013. pp. 3-17. DOI: 10.1007/978-1-4614-3794-9

[5] Johnson A. Deglutition. In: Gleeson M, editor. Brown's Otolarynology. Volume 1: Basic Sciences. 6th ed. Butterwoeth-Heinemann; 1997

[6] Ekberg O, Nylander G. Anatomy and Physiology, Dysphagia: Diagnosis and Treatment. New York: Springer Science \& Business Media; 2012. pp. 1-18. DOI: 10.1007/978-3-319-68572-4

[7] Matsuo K, Palmer JB. Oral phase preparation and propulsion: Anatomy, physiology, rheology, mastication, and transport. In: Shaker R, Belafsky PC, Postma GN, et al., editors. Principles of Deglutition: A Multidisciplinary Text for Swallowing and Its Disorders. New York: Springer Science \& Business Media; 2013. pp. 117-131. DOI: 10.1007/978-1-4614-3794-9

[8] Manly RS, Braley LC. Masticatory performance and efficiency. Journal of Dental Research. 1950;29:448-462. DOI: 10.1177/00220345500290040701
[9] Mowlana F, Heath MR, Van der Bilt A, Van der Glas HW. Assessment of chewing efficiency: A comparison of particle size distribution determined using optical scanning and sieving of almonds. Journal of Oral Rehabilitation. 1994;21:545-551. DOI: 10.1111/j.13652842.1994.tb01168

[10] Chauncey HH, Muench ME, Kapur KK, Wayler AH. The effect of the loss of teeth on diet and nutrition. International Dental Journal. 1984;34:98-104

[11] Helkimo E, Carlsson GE, Helkimo M. Chewing efficiency and state of dentition. A methodologic study. Acta Odontologica Scandinavica. 1978;36:33-41. DOI: $10.3109 / 00016357809026364$

[12] Akeel R, Nilner M, Nilner K. Masticatory efficiency in individuals with natural dentition. Swedish Dental Journal. 1992;16:191-198

[13] Naka O, Anastassiadou V, Pissiotis A. Association between functional tooth units and chewing ability in older adults: A systematic review. Gerodontology. 2012. DOI: 10.1111/ger.12016

[14] Tate GS, Throckmorton GS, Ellis E 3rd, Sinn DP. Masticatory performance, muscle activity, and occlusal force in preorthognathic surgery patients. Journal of Oral and Maxillofacial Surgery. 1994;52:476-481. DOI: 10.1016/0278-2391(94)90344-1

[15] Kapur K, Soman S. Masticatory performance and efficiency in denture wearers. The Journal of Prosthetic Dentistry. 1964;14:687-694. DOI: 10.1016/0022-3913(64)90203-3

[16] Pedersen AM, Bardow A, Jensen SB, Nauntofte B. Saliva and gastrointestinal functions of taste, 
mastication, swallowing and digestion. Oral Diseases. 2002;8:117-129. DOI: 10.1034/j.1601-0825.2002.02851.x

[17] Moore KL, Dalley AF, Agur AM. Clinically Oriented Anatomy. Baltimore, MD: Lippincott Williams \& Wilkins; 2013. pp. $981-1052$

[18] Young JL, Riordan MO, Goldstein JA, et al. What information do parents of newborns with cleft lip, palate, or both want to know? The Cleft Palate-Craniofacial Journal. 2001;38(1):55-58. DOI: 10.1597/1545-1569_2001_038_0055_ widpon_2.0.co_2

[19] Reid J, Kilpatrick N, Reilly S. A prospective, longitudinal study of the feeding skills in a cohort of babies with cleft conditions. The Cleft PalateCraniofacial Journal. 2006;43(6):702709. DOI: $10.1597 / 05-172$

[20] Reid J, Reilly S, Kilpatrick N. Sucking performance of babies with cleft conditions. The Cleft PalateCraniofacial Journal. 2007;33(3): 312-320. DOI: 10.1597/05-173

[21] Carlisle D. Feeding babies with cleft lip and palate. Nursing Times. 1998;94(4):59-60

[22] Gailey DG. Feeding infants with cleft and the postoperative cleft management. Oral and Maxillofacial Surgery Clinics of North America. 2016;28(2):153-159. DOI: 10.1016/j. coms.2015.12.003

[23] Cohen M, Marschall MA, Schafer ME. Immediate: Unrestricted feeding of infants following cleft lip and palate repair. The Journal of Craniofacial Surgery. 1992;3(1):30-32. DOI: 10.1097/00001665-199207000-00011

[24] Kim EK, Lee TJ, Chae SW. Effect of unrestricted bottle-feeding on early postoperative course cleft palate repair. The Journal of Craniofacial Surgery.
2009;20(2):1886-1888. DOI: 10.1097/

SCS.0b013e3181b2d1d1

[25] Trettene Ados S, Mondini CC, Marques IL, et al. Feeding children in the immediate perioperative period after palatoplasty: A comparison between techniques using a cup and spoon. Revista da Escola de Enfermagem da USP, REEUSP. 2013;47(6):1298-1304. DOI: 10.1590/ S0080-623420130000600007

[26] Hughes M, Lindup SW, et al. Does nasogastric feeding reduce distress after cleft palate repair in infants? Nursing Children and Young People. 2013;25(9):26-30. DOI: 10.7748/ ncyp2013.11.25.9.26.e324

[27] Hadadi AI, Al Wohaibi D, et al. Congenital anomalies associated with syndromic and non-syndromic cleft lip and palate. JPRAS Open. 2017;14:5-15. DOI: 10.1016/j.jpra.2017.06.001

[28] Rajion ZA, Al-Khatib AR, Netherway DJ, et al. The nasopharynx in infants with cleft lip and palate. International Journal of Pediatric Otorhinolaryngology. 2012;76(2):227234. DOI: $10.1016 /$ j.ijporl.2011.11.008

[29] Rajion ZA, Townsend GC, Netherway DJ, et al. The hyoid bone in malay infants with cleft lip and palate. The Cleft Palate-Craniofacial Journal. 2006;43(5):532-538. DOI: $10.1597 / 05-085$

[30] World Health Organization: Oral health. Available from: http://www.who. int/mediacentre/factsheets/fs318/en/ index.html [Accessed: March 2013]

[31] Marik PE, Kaplan D. Aspiration pneumonia and dysphagia in the elderly. Chest. 2003;124:328-336. DOI: 10.1378/ chest.124.1.328

[32] Yoneyama T, Yoshida M, Ohrui T, et al. Oral care reduces pneumonia in older patients in nursing homes. 
Journal of the American Geriatrics Society. 2002;50:430-433. DOI: 10.1046/j.1532-5415.2002.50106

[33] Sato J, Goto J, Harahashi A, et al. Oral health care reduces the risk of postoperative surgical site infection in inpatients with oral squamous cell carcinoma. Supportive Care in Cancer. 2011;19:409-416. DOI: 10.1007/ s00520-010-0853-6

[34] Hildebrandt GH, Dominguez BL, Schork MA, Loesche WJ. Functional units, chewing, swallowing, and food avoidance among the elderly. The Journal of Prosthetic Dentistry. 1997;77:588-595. DOI: $10.1016 /$ S0022-3913(97)70100-8

[35] Mishellany A, Woda A, Labas R, Peyron MA. The challenge of mastication: Preparing a bolus suitable for deglutition. Dysphagia. 2006;21:8794. DOI: $10.1007 / \mathrm{s} 00455-006-9014$

[36] Tamura F, Mizukami M, Ayano R, Mukai Y. Analysis of feeding function and jaw stability in bedridden elderly. Dysphagia. 2002;17:235-241. DOI: 10.1007/s00455-002-0063-6

[37] Furuta M, Komiya-Nonaka M, Akifusa S, et al. Interrelationship of oral health status, swallowing function, nutritional status, and cognitive ability with activities of daily living in Japanese elderly people receiving home care services due to physical disabilities. Community Dentistry and Oral Epidemiology. 2013;41:173-181. DOI: 10.1111/cdoe. 12000

[38] Yoshikawa M, Yoshida M, Nagasaki T, et al. Influence of aging and denture use on liquid swallowing in healthy dentulous and edentulous older people. Journal of the American Geriatrics Society. 2006;54:444-449. DOI: 10.1111/j.1532-5415.2005.00619

[39] Monaco A, Cattaneo R, Masci C, et al. Effect of ill-fitting dentures on the swallowing duration in patients using polygraphy. Gerodontology. 2012;29:e637-e644. DOI: 10.1111/j.1741-2358.2011.00536.x

[40] Hiiemae KM, Palmer JB. Food transport and bolus formation during complete feeding sequences on foods of different initial consistency. Dysphagia. 1999;14:31-42. DOI: 10.1007/ PL00009582

[41] Paganini-Hill A, White SC, Atchison KA. Dentition, dental health habits, and dementia: The leisure world cohort study. Journal of the American Geriatrics Society. 2012;60:1556-1563. DOI: 10.1111/j.1532-5415.2012.04064

[42] Cook IJ, Kahrilas PJ. AGA technical review on management of oropharyngeal dysphagia.

Gastroenterology. 1999;116:455-478.

DOI: 10.1016/S0016-5085(99)70144-7

[43] Liedberg B, Owall B. Masticatory ability in experimentally induced xerostomia. Dysphagia. 1991;6:211-213. DOI: $10.1007 /$ BF02493529

[44] Ship JA, Pillemer SR, Baum BJ. Xerostomia and the geriatric patient. Journal of the American Geriatrics Society. 2002;50:535-543. DOI: $10.1046 /$ j.1532-5415.2002.50123.x

[45] Kakudate N, Muramatsu T, Endoh M, et al. Factors associated with dry mouth in dependent Japanese elderly. Gerodontology. 2012. DOI: 10.1111/j.1741-2358.2012.00685

[46] Papas A, Singh M, Harrington D, et al. Stimulation of salivary flow with a powered toothbrush in a xerostomic population. Special Care in Dentistry. 2006;26:241-246. DOI: 10.1111/j.17544505.2006.tb01661

[47] Meningaud JP, Pitak-Arnnop P, Chikhani L, Bertrand JC. Drooling of saliva: A review of the etiology and management options. Oral Surgery, 
Oral Medicine, Oral Pathology, Oral Radiology, and Endodontics. 2006;101:48-57. DOI: 10.1016/j. tripleo.2005.08.018

[48] Alotaibi N, Cloutier L, Khaldoun E, Bois E, Chirat M, Salvan D. Criteria for admission of odontogenic infections at high risk of deep neck space infection. European Annals of Otorhinolaryngology, Head and Neck Diseases. 2015;132(5):261-264. DOI: 10.1016/j.anorl.2015.08.007

[49] Flynn TR, Shanti RM, Levi MH, Adamo AK, Kraut RA, Trieger N. Severe odontogenic infections, part 1 :

Prospective report. Journal of Oral and Maxillofacial Surgery. 2006;64:10931103. DOI: 10.1016/j.joms.2006.03.015

[50] Beck HJ, Salassa JR, McCaffrey TV, Hermans PE. Life-threatening soft tissue infections of the neck. The Laryngoscope. 1984;94:354-362. DOI: 10.1288/00005537-198403000-00012

[51] Larawin V, Naipao J, Dubey SP. Head and neck space infections. Otolaryngology-Head and Neck Surgery. 2006;135:889-893. DOI: 10.1016/j.otohns.2006.07.007

[52] Poeschl PW, Spusta L, Russmueller G, Seemann R, Hirschl A, Poeschl E, et al. Antibiotic susceptibility and resistance of the odontogenic microbiological spectrum and its clinical impact on severe deep space head and neck infections. Oral Surgery, Oral Medicine, Oral Pathology, Oral Radiology, and Endodontics. 2010;110:151-156. DOI: 10.1016/j. tripleo.2009.12.039

[53] Colmenero Ruiz C, Labajo AD, Yañez Vilas I, Paniagua J. Thoracic complications of deeply situated serous neck infections. Journal of CranioMaxillo-Facial Surgery. 1993;21:76-81. DOI: 10.1016/S1010-5182(05)80151-9

[54] Levitt GW. Cervical fascia and deep neck infections. The
Laryngoscope. 1970;80:409-435. DOI: 10.1288/00005537-197003000-00004

[55] Fursta IM, Ersil P, Caminiti M. A rare complication of tooth abscess Ludwig's angina and mediastinitis. Journal of the Canadian Dental Association. 2001;67(6):324-327

[56] Marcus BJ, Kaplan J, Collins KA. A case of Ludwig angina: A case report and review of the literature. The American Journal of Forensic Medicine and Pathology. 2008;29(3):255-259. DOI: 10.1097/PAF.0b013e31817efb24

[57] Bross-Soriano D, Arrieta-Gomez JR, Prado-Calleros H, Schimelmitz-Idi J, Jorba-Basave S. Management of Ludwig's angina with small neck incisions: 18 years experience. Otolaryngology and Head and Neck Surgery. 2004;130(6):712-717. DOI: 10.1016/j.otohns.2003.09.036

[58] Moreland LW, Corey J, McKenzie R. Ludwig's angina. Report of a case and review of the literature. Archives of Internal Medicine. 1988;148(2):461-466. DOI: 10.1001/ archinte.1988.00380020205027

[59] Jimenez Y, Bagan JV, Murillo J, Poveda R. Odontogenic infections. Complications. Systemic manifestations. Medicina Oral, Patología Oral y Cirugía Bucal. 2004;9(143-147):139-143

[60] Candamourty R, Venkatachalam S, Babu MR, Kumar GS. Ludwig's angina-An emergency: A case report with literature review. Journal of Natural Science, Biology and Medicine. 2012;3:2206-2208. DOI: 10.4103/0976-9668.101932

[61] Scully C, Langdon J, Evans J. Marathon of eponyms:12 Ludwig angina. Oral Diseases. 2010;16(5):496-497. DOI: 10.1111/j.1601-0825.2009.01544.x

[62] Wills PI, Vernon RP. Complications of space infections of the head and neck. 
Maxillofacial and Oral Aspects of Dysphagia

DOI: http://dx.doi.org/10.5772/intechopen.89751

Laryngoscope. 1981;91:1129-1136. DOI: 10.1288/00005537-198107000-00010

[63] Vieira F, Allen SM, Stocks RSM, Thompson JW. Deep neck infections. Otolaryngologic Clinics of North America. 2008;12:459-483. DOI: 10.1016/j.otc.2008.01.002

[64] Jaworski CP, Koudelka BM, et al. Acute necrotizing ulcerative gingivitis in a case of systemic lupus erythematosus. Journal of Oral and Maxillofacial Surgery. 1985;43(1):43-46. DOI: 10.1016/S0278-2391(85)80012-4

[65] Francis TC. Recurrent aphthous stomatitis and Behçet's disease: A review. Oral Surgery, Oral Medicine, and Oral Pathology. 1970;30(4):476-487. DOI: 10.1016/0030-4220(70)90161-1

[66] Barker GR. Bilateral ranula. International Journal of Oral Surgery. 1984;13:352-354. DOI: 10.1016/ S0300-9785(84)80044-7

[67] Gupta A, Karjodka FR. Plunging ranula: A case report. Dentistry. 2011:15. DOI: $10.5402 / 2011 / 806928$

[68] Suresh BV, Vora KS. Huge plunging ranula. The Journal of Oral and Maxillofacial Surgery. 2012;11:487. DOI: 10.1007/s12663-010-0154-0

[69] Davison MJ, Morton RP, McIvor NP. Plunging ranula: Clinical observations. Head and Neck. 1998;20:63-68. DOI: $10.1002 /$ (SICI)1097-0347(199801)20:1<63::AIDHED10>3.0.CO;2-Q

[70] Kaftan B, Snyder HS. Lingual artery hematoma resulting in upper airway obstruction. The Journal of Emergency Medicine. 1991;9:421. DOI: 10.1016/0736-4679(91)90212-X

[71] Saah D, Elidan J, Braverman I, et al. Traumatic macroglossia. The Annals of Otology, Rhinology, and Laryngology.
1993;102:729-773. DOI: 10.1177/000348949310200915

[72] Cohen AF, Warman SP. Upper airway obstruction secondary to warfarin induced sublingual haematoma. Archives of Otolaryngology Head and Neck Surgery. 1989;115(6):718-720. DOI: 10.1001/ archotol.1989.01860300072020

[73] Williams PJ, Jani P, McGlashan J. Lingual haematoma following treatment with streptokinase and heparin; anaesthetic management. Anaesthesia. 1994;49:417-418. DOI: 10.1111/j.13652044.1994.tb03477.x

[74] Takeuchi M, Shikimori M, Kaneda T. Life-threatening sublingual haematoma in a severely hemophilic patient with factor VIII inhibitor. Journal of Oral and Maxillofacial Surgery. 1986;44:401-403. DOI: 10.1016/S0278-2391(86)80038-6

[75] Massey B et al. Case report: Traumatic lingual hematoma. Trauma Case Reports. 2019;20:100177. DOI: 10.1016/j.tcr.2019.100177

[76] Tarakji B, Nassani MZ et al. Factors associated with hematoma of the floor of the mouth after placement of dental implants. The Saudi Dental Journal. 2012;24(1):11-15. DOI: 10.1016/j. sdentj.2011.11.003

[77] Esmer E, Delank KS, Siekmann H, Schulz M, Derst P. Facial injuries in polytrauma-Which injuries can be expected? A retrospective evaluation from the TraumaRegister DGU. Notfall Rettungsmedizin. 2016;19:92-98

[78] Stephan K, Huber S, Häberle S, Kanz KG, Bühren V, van Griensven M, et al. Spinal cord injury-Incidence, prognosis, and outcome: An analysis of the TraumaRegister DGU. The Spine Journal. 2015;15:1994-2001. DOI: 10.1016/j.spinee.2015.04.041 
[79] Heffner JE. Swallowing complications after endotracheal extubation. Chest. 2010;137:509-510. DOI: $10.1378 /$ chest.09-2477

[80] Gealock AJ Jr. The flared mandible sign of the flail mandible. Radiology. 1976;117:299-300. DOI: 10.1148/119.2.299

[81] Zachariades N. Laryngeal incompetence following facial trauma. Journal of Oral and Maxillofacial Surgery. 1985;43(8):638-639. DOI: 10.1016/0278-2391(85)90139-9

[82] Tiwary P, Sahoo N, Thakral A, Ranjan U. Styloid process fracture associated with maxillofacial trauma: Incidence, distribution, and management. Journal of Oral and Maxillofacial Surgery. 2017;75(10):21772182. DOI: 10.1016/j.joms.2017.06.016

[83] Ortigara GB et al. Association between trismus and dysphagia-related quality of life in survivors of head and neck cancer in Brazil. Oral Surgery, Oral Medicine, Oral Pathology, Oral Radiology. 2019;128(3):235-224. DOI: 10.1016/j.oooo.2019.05.009

[84] Ferlay J, Soerjomataram I, Dikshit R, et al. Cancer incidence and mortality world-wide: Sources, methods and major patterns in GLOBOCAN 2012. International Journal of Cancer. 2015;136:359-386. DOI: 10.1002/ ijc. 29210

[85] Torre LA, Bray F, Siegel RL, Ferlay J, Lortet-Tieulent J, Jemal A. Global cancer statistics, 2012. CA: a Cancer Journal for Clinicians. 2015;65:87-108. DOI: $10.3322 /$ caac. 21262

[86] Nund RL, Ward EC, Scarinci NA, et al. The lived experience of dysphagia following nonsurgical treatment for head and neck cancer. International Journal of Speech-Language Pathology. 2014;16:282. DOI: 10.3109/17549507.2013.861869
[87] De Felice F, de Vincentiis M, Luzzi V, Magliulo G, Tombolini M, Ruoppolo G, et al. Late radiationassociated dysphagia in head and neck cancer patients: Evidence, research and management. Oral Oncology. 2018;77:125-130. DOI: 10.1016/j. oraloncology.2017.12.021

[88] Chen S-C et al. Swallowing ability and its impact on dysphagia-specific health-related QOL in oral cavity cancer patients post-treatment. European Journal of Oncology Nursing. 2018;36:89-94. DOI: 10.1016/j. ejon.2018.07.002

[89] Huang Z-s, Chen W-l, Huang Z-q, Yang Z-h. Dysphagia in tongue Cancer patients before and after surgery. Journal of Oral and Maxillofacial Surgery. 2016;74(10):2067-2072. DOI: 10.1016/j.joms.2016.03.031

[90] Langendijk JA, Doornaert P, Rietveld $\mathrm{DH}$, et al. A predictive model for swallowing dysfunction after curative radiotherapy in head and neck cancer. Radiotherapy and Oncology. 2009;90:189. DOI: 10.1016/j. radonc.2008.12.017 
Section 5

Radiation-Related Dysphagia 



\title{
Radiation-Related Dysphagia: From Pathophysiology to Clinical Aspects
}

\author{
Stefano Ursino, Paola Cocuzza, Stefania Santopadre, \\ Fabiola Paiar and Bruno Fattori
}

\begin{abstract}
In Western countries, head and neck cancers (HNCs) account for about $5 \%$ of all tumors. Due to tumor locations at the aero-digestive crossroad, patients frequently suffer from swallowing dysfunction caused both by primary cancer (baseline dysphagia) and cancer therapies (treatment-related dysphagia). In this regard, radiation-induced dysphagia represents a real "Achille's heel" which historically occurs in more than 50\% of patients and can lead to a malnutritional status and an increased risk of aspiration pneumonia. In fact radiotherapy, by restricting the driving pressure of the bolus through the pharynx and/or limiting the opening of the cricopharyngeal muscle, leads to a post-swallowing pharyngeal residue that may spill into the airway causing ab ingestis pneumonia. On the contrary, an organ preservation strategy should provide both the highest tumor control probability (TCP) and the minimum function impairment with the subsequent maximum therapeutic index gain. In this regard, intensity-modulated RT (IMRT) might reduce the probability of postradiation dysphagia by producing concave dose distributions with better avoidance of several critical structures, such as swallowing organs at risk (SWOARs), which might result in better functional outcomes. Similarly, a prompt swallowing rehabilitation provided before, during, and soon after radiotherapy plays an important role in improving oncologic swallowing outcomes.
\end{abstract}

Keywords: swallowing, dysphagia, intensity and modulated radiotherapy, chemotherapy, aspiration, videofluoroscopy

\section{Introduction}

Nowadays, radiotherapy (RT) alone or most frequently combined with chemotherapy (RCT) is considered a valid alternative treatment to surgery for patients affected by head and neck cancers (HNCs) in order to preserve the deglutition organ $[1,2]$. Historically, conventional RT has been burdened by severe and potentially "life threatening" toxicity that limited the delivery of high tumor radiation dose and in most cases affected the final treatment result [3-6]. In this regard, radiation-induced dysphagia, as a final multifactorial side effect often requiring enteral nutrition, has always represented a real "Achille's heel" occurring in more than $50 \%$ of patients and leading to a malnutritional status, increased risk of aspiration pneumonia, and long-term percutaneous endoscopic gastrostomy (PE) tube placement positioning [7-10]. 
Moreover, eating together is a defining social activity among family, friends, and colleagues. For most people, the ability to enjoy eating helps to define quality of life (QoL), whereas labored swallowing, prolonged eating times, and the limited range of foods that can be swallowed can lead to disruption of relationships and social isolation [11].

Indeed, in the last decades, an improvement of oncologic outcomes such as local control and overall survival has come from an increasing use of the more aggressive altered fractionation RT schedules and the frequent use of RCT sometimes preceded by induction chemotherapy $[1,12]$. Therefore, the common use of high intensified organ preservation strategies has resulted in "potentially" high rates of swallowing dysfunction prompting to consider postradiation dysphagia as the real "barrier to winning the battle of HNC" [13].

\section{Basic concepts of oropharyngeal swallowing physiology}

Swallowing is a functional complex process, requiring the involvement and perfect sequential coordination of more than 30 pairs of muscles and 6 cranial nerves (V, VII, IX, X,XII) [14-16].

In this regard, pharynx plays a crucial role as an aero-digestive crossroad region both at its upper (naso and oropharynx) and lower part (hypopharynx and larynx) to ensure the constant and effective protection of the airways from the entrance of the bolus together with the safe passage of the bolus into the upper esophagus. This process implies a very complex anatomical change of the pharyngeal tract from a respiratory to a deglutitory configuration to fastly return to the respiratory one in less than a second [17-19].

This process starts with the passage of the bolus through the palatoglossus sphincter (the beginning of the involuntary deglutition) and ends with the relaxing of the cricopharyngeal muscle and the passive opening of the upper esophageal sphincter that moves the bolus into the lower digestive tract (pharyngeal phase). This phase can be further divided into three different subphases: oropharyngeal, pharyngeal, and pharyngo-esophageal phase.

The oropharyngeal subphase corresponds to the activation of "trigger area" located between the anterior palatine pillars, palatine veil and the base of tongue which is innervated by the IX and X cranial nerves. This subphase is mostly characterized by the uplift of the palatine veil which contacts with the posterior pharyngeal wall to close the nasopharynx avoiding the nasal reflux of the bolus (or regurgitation) and by the contraction of the hyoglossus muscle which causes the tilt of the base of tongue favoring the slip of the bolus into the pharynx [20].

The Pharyngeal phase is characterized by the reinforcement of the nasopharynx sealing for the anterior movement of the posterior pharyngeal wall due to the contraction of superior pharyngeal muscle, by the closure of glottic larynx to protect the lower respiratory tract through the activation of superior laryngeal nerve and by the progression of the bolus to the superior esophageal sphincter.

This is the most crucial phase of deglutition as it corresponds to the crossing and overcoming of the bolus of the aero-digestive crossroad.

The closure of the glottic larynx represents the principle mechanism of inferior airways protection which sequentially starts with the true vocal cords adduction (contraction of inferior and medium tyroarytenoid muscles, lateral cricoarytenoid muscles, and interarytenoid muscles), continues with the false vocal cords adduction (tyroarytenoid muscles contraction), and ends with the epiglottis retroflexion [21]. In this regard, the glottic closure is recognized as the main mechanism to protect inferior airways, the retroflexion of epiglottis being the less important. 
Specifically, the protection of inferior airways is mostly guaranteed by the early closure of the glottic larynx (due to the true and false vocal cords adduction) together with the combination of the anterior and upper movement of hyolaryngeal complex (due to the contraction of the suprahyoid musculature and inhibition of subhyoid musculature) and the back-forward movement of the base of tongue (due to the contraction of stylo and hyoglossus muscles) that contributes to protect the laryngeal aditus from the spilling of the bolus [17, 22-26].

Indeed, the progression of the bolus through the pharynx is sequentially related to the tongue base retraction and the pharyngeal peristalsis combined with the force of gravity and the downward aspiration of the bolus. Specifically, the tongue base retraction just precedes the pharyngeal peristalsis that is characterized by a craniocaudal stripping wave that propagates toward the hypopharynx and is performed by the sequential contraction of superior, medium, and inferior constrictor muscles. The force of gravity also plays an important role as well as the downward aspiration that is produced in the hypopharynx by the hyolaryngeal complex elevation $[19,27,28]$.
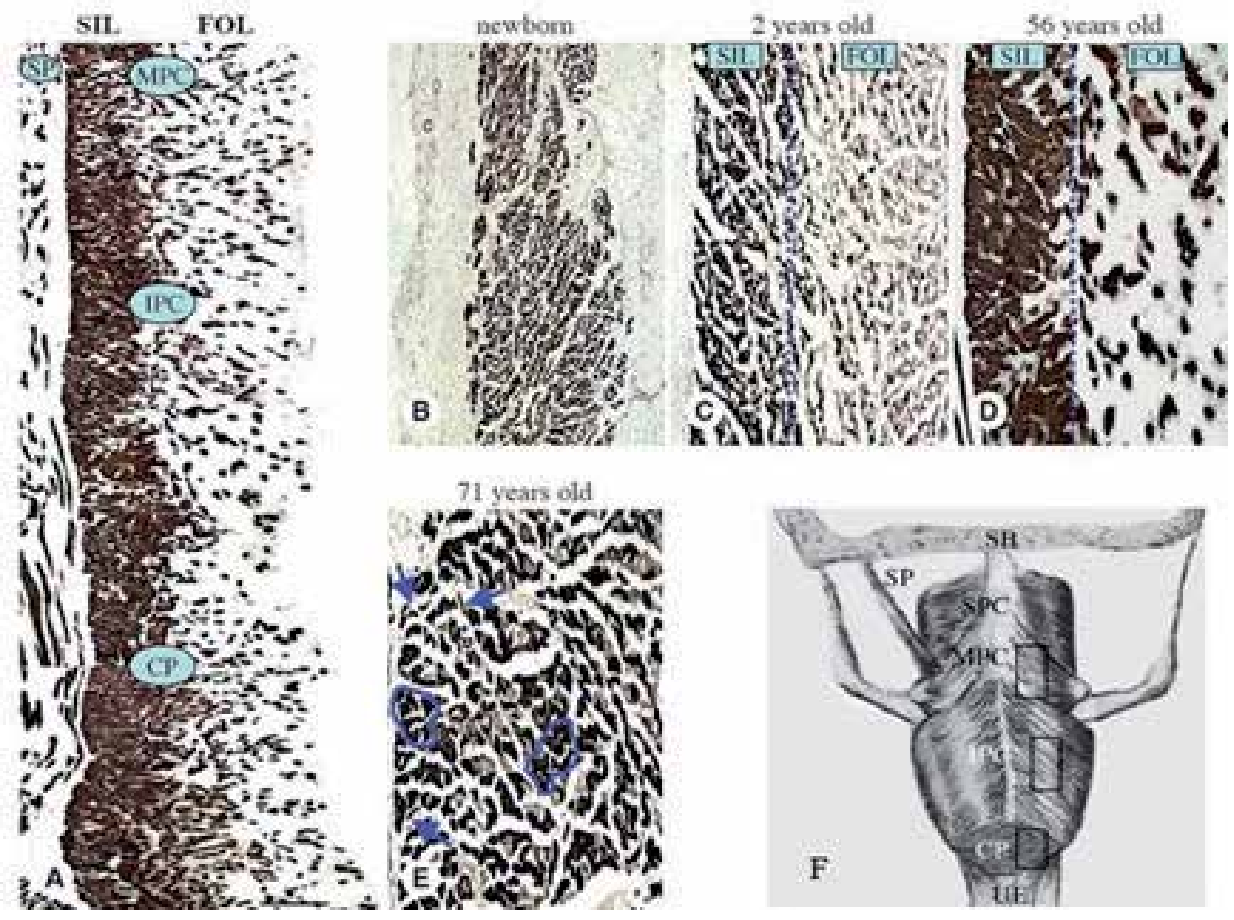

Figure 1.

Developmental (humans at different ages) changes in PC fiber layers. (A) Low-power photomicrographs of immunocytochemically stained cross sections from adult human MPC, IPC, and CP muscles. Scale bar-1 $\mathrm{mm}$. (B)-(G) High-power photomicrographs of IPC muscles of (B) human newborn, (C) 2-year-old human, (D) adult human, and (E) elderly human, (Scale bar-100 $\mu \mathrm{m}$ ). All sections were incubated with monoclonal antibody NOQ7-5-4D specific to slow type I myosin heavy chain (MHC) isoform by avidin-biotin complex method. In all sections, slow type I fibers were stained dark, whereas fast type II fibers were stained light. Slow inner layer (SIL), which contained predominantly slow type I fibers, stained dark, whereas fast outer layer (FOL), which contained primarily fast type II fibers, stained light. Note that layered structure of PCs was identified in 2-year-old human (C) and normal adult humans $(A, D)$, but not in human newborn $(B)$ Also note that PC fiber layers in elderly human $(E)$ were obscured because of fast-to-slow MHC transformation that occurred mainly in FOL of PCs. In addition, fiber type grouping (circled fibers) and fiber atrophy (arrows) were apparent in aged $(E)$ muscle $(F)$. Schematic of human pharynx (posterior view) illustrates arrangement of pharyngeal constrictor $(P C)$, cricopharyngeus $(C P)$, and stylopharyngeus $(S P)$ muscles and tissue sampling sites (enclosed regions) for immunocytochemistry. IPC, inferior PC; MPC, middle PC; SB, skull base; SPC, superior PC; and UE, upper esophagus. 
Lastly, the pharyngo-esophageal phase is characterized by the opening of the superior esophageal sphincter due to both the hyolaryngeal complex elevation and the cricopharyngeal muscle relaxation, the latter produced by the reduction of basal tonic activity of the vagus nerve [29].

Finally, after the passsage of the bolus into the upper esophagus, the superior esophageal sphincter closes, the hyolaryngeal complex lowers down to the baseline position and the glottic larynx opens leading the pharynx to the baseline respiratory conformation.

The neuronal pathways also play a central role in the swallowing process.

Sensory inputs from physicochemical properties of the bolus (taste, pressure, temperature, and nociceptive somatic stimuli) from oropharynx and larynx are transported through cranial nerves V, VII, IX, and X to the central pattern generator within the nucleus tractus solitarius, where they are integrated and organized with information from the cortex. The somatic sensorial input required for proper swallowing is perceived by the lingual branches of the trigeminal and glossopharyngeal nerves, the pharyngeal branches of the glossopharyngeal and vagus nerves, and the laryngeal branch of the vagus nerve. The swallow response is elicited in the brain stem swallowing center, which receives strong modulating inputs from both the oropharynx and the cortex [30].
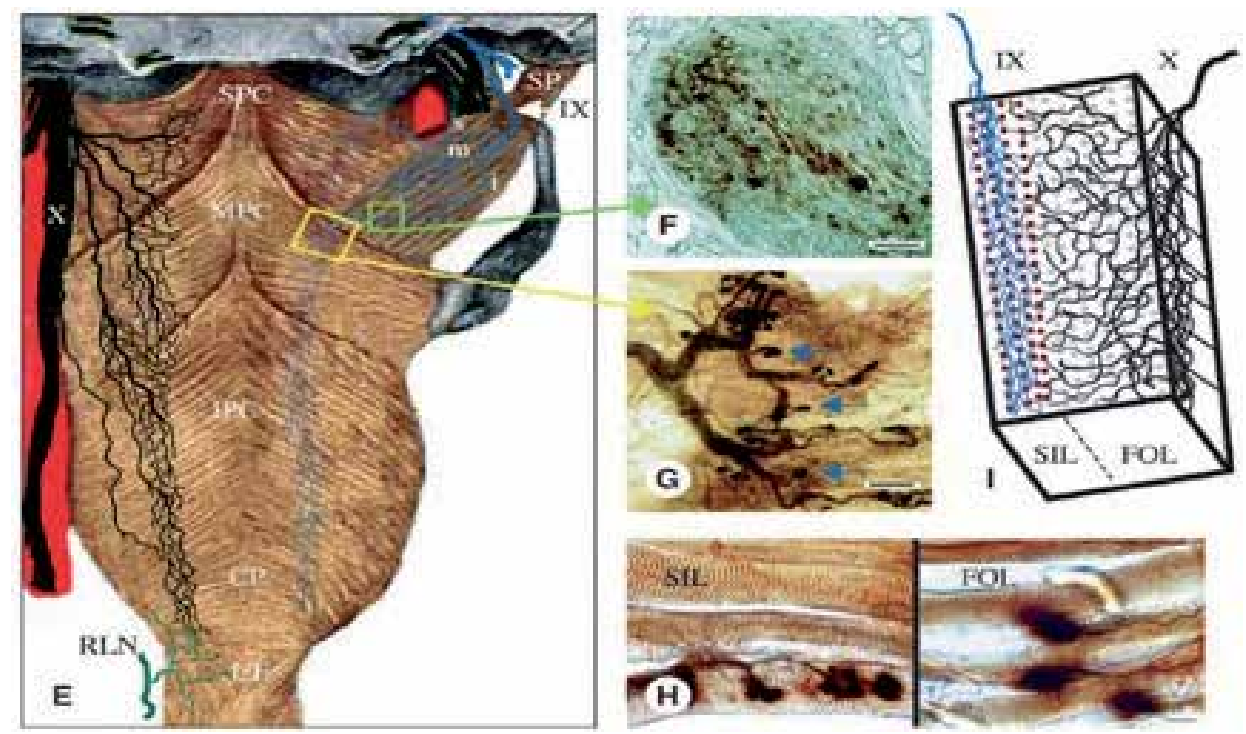

Figure 2.

(A) Schematic of adult human pharynx (posterior view) shows motor innervation of FOL and SIL in PCs. Note that FOL is supplied by Ph-X (left side), whereas SIL is innervated by Ph-IX (right side). After leaving skull through jugular foramen, nerve IX (right side) is subdivided into sensory (cut end) and motor (arrow) divisions. Motor division of Ph-IX gives off three branches—superior $(s)$, middle $(m)$, and inferior (i)—which supply SIL of SPC (dotted lines), stylopharyngeus (SP) muscle, and SIL in MPC, IPC, and CP muscles (dotted lines), respectively. Boxes indicate sampling sites for nerve segment from inferior branch of motor division of Ph-IX (small box) and its innervating muscle tissue (large box). $(B, C)$ Histochemical evidence for motor contribution of IX to SIL in PCs. (B) Karnovsky-Roots AChE-stained cross section of nerve segment sampled from inferior branch of Ph-IX (small box in $(E))$. Note that this nerve branch contained motor axons (brown staining). Scale bar-100 $\mu \mathrm{m}$. (C) Whole mount acetylcholinesterase and silver (AChE-Ag)-stained SIL in MPC muscle innervated by nerve terminals (large box in $(A))$ distal to sampled nerve segment. Note that terminals of this nerve branch innervated motor end plates (MEPs; arrows) on SIL muscle fibers. Scale bar-100 $\mu \mathrm{m}$. (D) High-power view of AChE-Ag-stained MEPs shows types of MEPs (en plaque or en grappe) and preterminal branching patterns (single or multiple) of axons innervating MEPs. Note that single SIL fiber had multiple en grappe MEPs (left), whereas most FOL fibers (right) had en plaque MEPs with single preterminal axon. Scale bar-20 $\mu \mathrm{m}$. (E) Schematic illustration of cross section from PC muscle in (A) shows intramuscular distribution patterns of nerve IX innervating SIL and nerve X innervating FOL in muscle. 
Besides, the histological and biochemical structure of the pharynx is considered of prior importance for the understanding of radiation-related swallowing damage as well as baseline deglutition impairment in elderly patients. Adult human pharynx is divided in two distinct and separate layers, an inner one (slow inner layer or SIL) that is innervated by the IX cranial nerve and an outer one (fast outer layer or FOL) that is innervated by the $\mathrm{X}$ cranial nerve. The first one is composed by a high prevalence of myofibers containing slow-twitch myosin (Type I) characterized by a slow time contraction, high mitochondrial density, and high oxidative capacity, and is mostly responsible for the tone, stiffness of the pharynx, and fine adjustments; whereas the second one is composed by a high prevalence myofibers containing fast-twitch myo$\sin$ (Type IIb) characterized by a fast contraction, low mitochondrial density, and low oxidative capacity and is mostly responsible for the contraction of the pharynx. Based on the fiber type and response to radical oxygen species, muscles with the highest glycolytic capacity (Type IIB) are most at risk for radiation damage. The immunohystochemical analysis of the pharynx has shown that the ratio between the width of the two layers (SIL/FOL) changes in a craniocaudal direction approximately from 2:1 in the cranial portion to 1:2 in the caudal portion of the pharynx. A physiological transformation process of the fast fibers into slow ones in the outer layer (also known as "fast to slow transformation") has been shown with the aging process reporting an overall $32 \%$ and $73 \%$ representation of type I fibers in the outer layer of the adult and elderly, respectively [31-33] (Figures 1 and 2).

\section{Pathophysiology of postradiation swallowing impairment}

Generally, based on the above reported mechanisms, anything that restricts the craniocaudal driving pressure, including the back-forward movement of the base of tongue, or impairs the hyolaryngeal complex elevation, or limits the cricopharyngeal muscle and/or superior esophageal sphincter opening, leads to post-swallowing pharyngeal residue that may spill into the airways. Thus, the post-swallowing aspiration is the risky consequence of a severe radiation-related dysphagia with the subsequent life-threatening risk of aspiration pneumonia (Figure 3). In this regard, the maintenance of cough reflexes is considered essential to avoid the spillage of the bolus below the vocal cords and prevent silent aspiration [11].

Moreover, a pathophysiological neuromuscular vicious circle can be described regarding postradiation dysphagia mechanism mainly consisting in a selective loss of the more radiosensitive type IIb myofibers together with a damage of peripheral nerves that innervate the swallowing musculature [29, 34, 35]. In the early phase

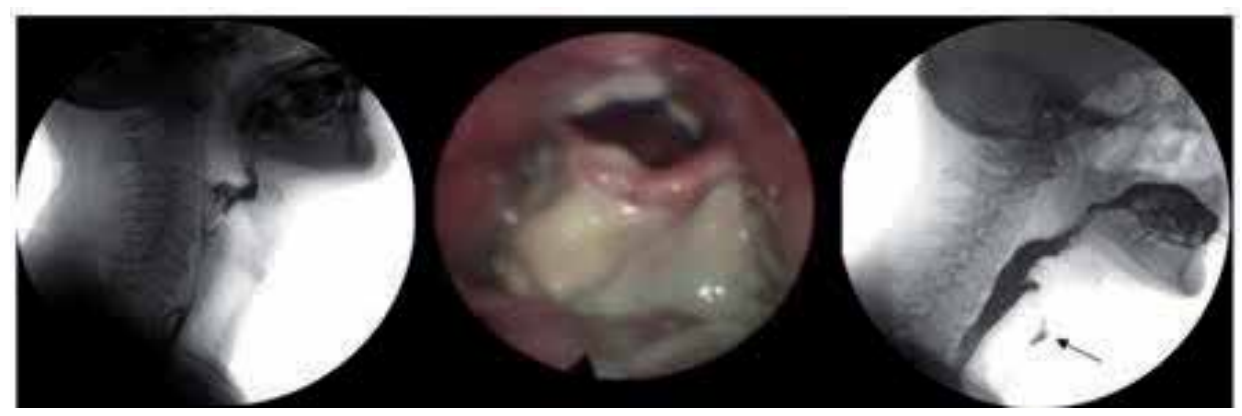

Figure 3.

Example of post-swallowing aspiration in an oropharyngeal cancer patient after $R T$. (A) Severe pharyngeal residue on Videofluoroscopy, $(B)$ severe pharyngeal residue both on glossoepiglottic folds and pyriform sinuses on fiberoptic endoscopic evaluation of swallowing and (C) aspiration on videofluoroscopy (black arrow). 
(during and immediately after radiotherapy), the neuropathic radiation damage usually arises from the breakdown of epithelium lining of pharyngolaryngeal mucosa, triggering a cascade of inflammatory mediators (i.e., cytokines, neuropeptides, and glutamate signals) that cause significant pain and discomfort and result in persistent sensory deficit $[36,37]$. In the late phase (after months from radiotherapy), the replacement of muscular fibers with fibrotic tissue, characterized by loss of vascularity and matrix disorganization that disrupts well-defined compartmentalized structures as well as excessive collagen deposit that entraps nerve trunks or alters the vascular network between or within the nerve tracts, leads to muscular and neurological deficits $[38,39]$. Then, the degeneration of muscular fibers probably related to the disuse of swallowing muscles (oral intake strongly declines during and immediately after radiotherapy) contributes to the transformation of fibrosis to atrophy leading to the final fibroatrophic damage [40].

Hyposensitivity and pharyngeal hypo/dysmotility are the clinical consequences of the neuromuscular radiation damage. Therefore, patients usually suffer from reduced sensory inputs (i.e., bolus size and consistencies) that may lead to "intradeglutitory" silent aspiration or impaired bolus movement through the pharynx tube with a consequent "post-swallowing" pharyngeal residue [38].

On the other hand, preclinical data clearly show that with aging, the number of satellite cells for myofiber decreases and those cells that remain exhibit a limited potential of regeneration. Also chronic inflammation and reactive oxygen species, such as those produced after irradiation, have been implicated in this aged effect.

However, endurance exercise has been reported to restore regenerative potential through changes in satellite cell number and function. As a matter of fact, in the study by Schadrach and Wagers $[41,42]$, the potential benefit of endurance exercise on satellite cells has been proven.

Myofibers of the inferior limb muscles were then isolated from exercised and sedentary mice, both young and old, and used for monitoring satellite cell numbers, and an expansion of the satellite cell pool in the exercised groups was compared with the sedentary one, regardless of the age (young and old).

\section{Clinical aspects}

\subsection{The role of preventive swallowing exercises}

Preradiation prophylactic swallowing therapy may be beneficial due to the upregulation of antioxidant enzymes and the enhancement of mitochondrial activity to increase the muscle fatigue resistance, as emerged from preclinical experiments [43-47]. In fact, it is crucial for myofibers of swallowing muscles to have efficient antioxidant capabilities to fight radiation-induced ROS that would, otherwise, cause irreversible damages.

Besides, atrophy is a consequence of both alterations of muscle proteins synthesis resulting in loss of muscle mass and the reduction of renewal of stem cells after radiotherapy [48-51].

Again, this knowledge might suggest that the initiation of the prescribed prophylactic swallowing exercises (i.e., before, concomitantly, or immediately following treatment) might improve functional swallowing outcomes [51-54].

Therefore, despite a strong preclinical rationale for the use of prophylactic strength-based exercises as well as to maintain oral intake throughout the entire radiation treatment to prevent or reduce the occurrence of radiation-induced dysphagia, data from the literature have some methodological concerns such as heterogeneity in the prescribed exercise regimen, in the tumor site/stage and the onset of intervention. 
However, a recent study by Hutcheson et al. [55] analyzed the independent effect of maintaining oral intake and proactive swallowing therapy in patients who underwent radiotherapy or chemoradiation for pharyngeal cancers. More specifically, the primary independent variables were:

1. Per oral nutrition status at the end of treatment (defined as NPO (nothing by mouth) and fully PEG tube dependent; partially PO, PEG tube supplemented by consistent daily oral intake; fully PO, $100 \%$ oral intake regardless of dietary level) and

2. Self-reported swallowing exercises adherence (adherent versus nonadherent). The results reported a greater proportion of patients who returned to a regular diet at 2 years for those who performed exercises and maintained full PO intake compared to those partial PO and adherent to exercises $(\mathrm{p}=0.02)$ and those full PO and nonadherent to exercises $(\mathrm{p}=0.02)$. Likewise, a significantly higher persistence of PEG-tube dependence was reported in those NPO and nonadherent to exercises compared to those part PO and adherent to exercises ( $39 \%$ versus $6 \% ; \mathrm{p}=0.03$ ). Besides, a previous work by CarnabyMann et al. [54] proved a superior maintenance of swallowing musculature structure (determined by $\mathrm{T} 2$-weighted magnetic resonance imaging) and a

\begin{tabular}{|c|c|c|}
\hline Maneuver & Indications & Rationgle \\
\hline Supraginttic sw'sllow & $\begin{array}{l}\text { Reduced airways clnzure } \\
\text { Aspiration during swallow }\end{array}$ & 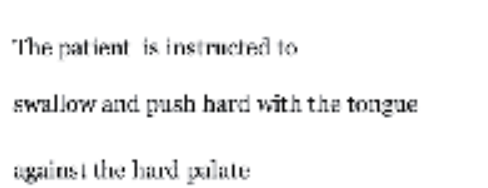 \\
\hline 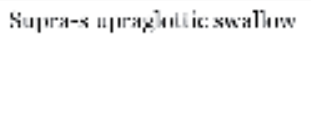 & $\begin{array}{l}\text { Kesluc:ed ainways flusure } \\
\text { Aspiration before and during } \\
\text { awallow }\end{array}$ & 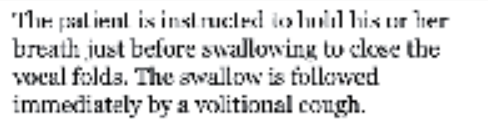 \\
\hline Werniteloghtn maneiner & 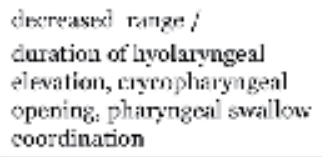 & $\begin{array}{l}\text { The prat irent hoids the latyrix in an elesated } \\
\text { pusilion at the peak of hyolayngeal elevalion }\end{array}$ \\
\hline Ettortful swallow & $\begin{array}{l}\text { vulloxulur residue } \\
\text { Keduced hase of tongue } \\
\text { rel ratinu" }\end{array}$ & $\begin{array}{l}\text { The putient is instructed to swallow und push } \\
\text { hard with the tongue againgt the hard palate }\end{array}$ \\
\hline Massiko & $\begin{array}{l}\text { Roduced base of tonguc } \\
\text { strenght } \\
\text { Keduced phinyrixeai } \\
\text { cuntriction }\end{array}$ & 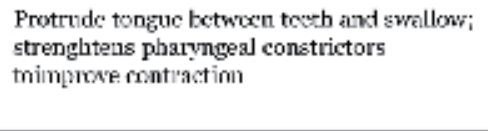 \\
\hline S.aker & $\begin{array}{l}\text { Upper esophageal sphincter } \\
\text { dysfunction } \\
\text { Roduced anterior and } \\
\text { supcrior movencut of the } \\
\text { byolarygeal conplex }\end{array}$ & 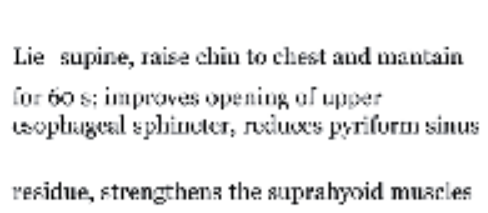 \\
\hline
\end{tabular}

Table 1.

Characteristics of swallowing exercises. 
better swallowing functional ability in patients who completed the program of swallowing exercises compared with those who did not.

Rehabilitation swallowing exercises in irradiated patients mainly consist of interventions aimed to reinforce supra-hyoid musculature (Mendelsohn maneuver), airway closure capability (supraglottic and super-supraglottic maneuver), base of tongue retraction (effortful swallow and Masako maneuver), and cricopharyngeus muscle opening (Shaker maneuver). A summary of the swallowing exercises advised in patients undergone to RT is reported in Table 1.

\subsection{Intensity and modulated radiotherapy (IMRT) as a strategy to reduce swallowing dysfunction}

An organ preservation strategy such as a radiation-based treatment should provide both the highest tumor control probability (TCP) and the minimum function impairment with the subsequent maximum therapeutic index gain [56].

In this regard, in the last few decades, the advancement of new treatment technologies, such as intensity and modulated radiotherapy (IMRT), has shown

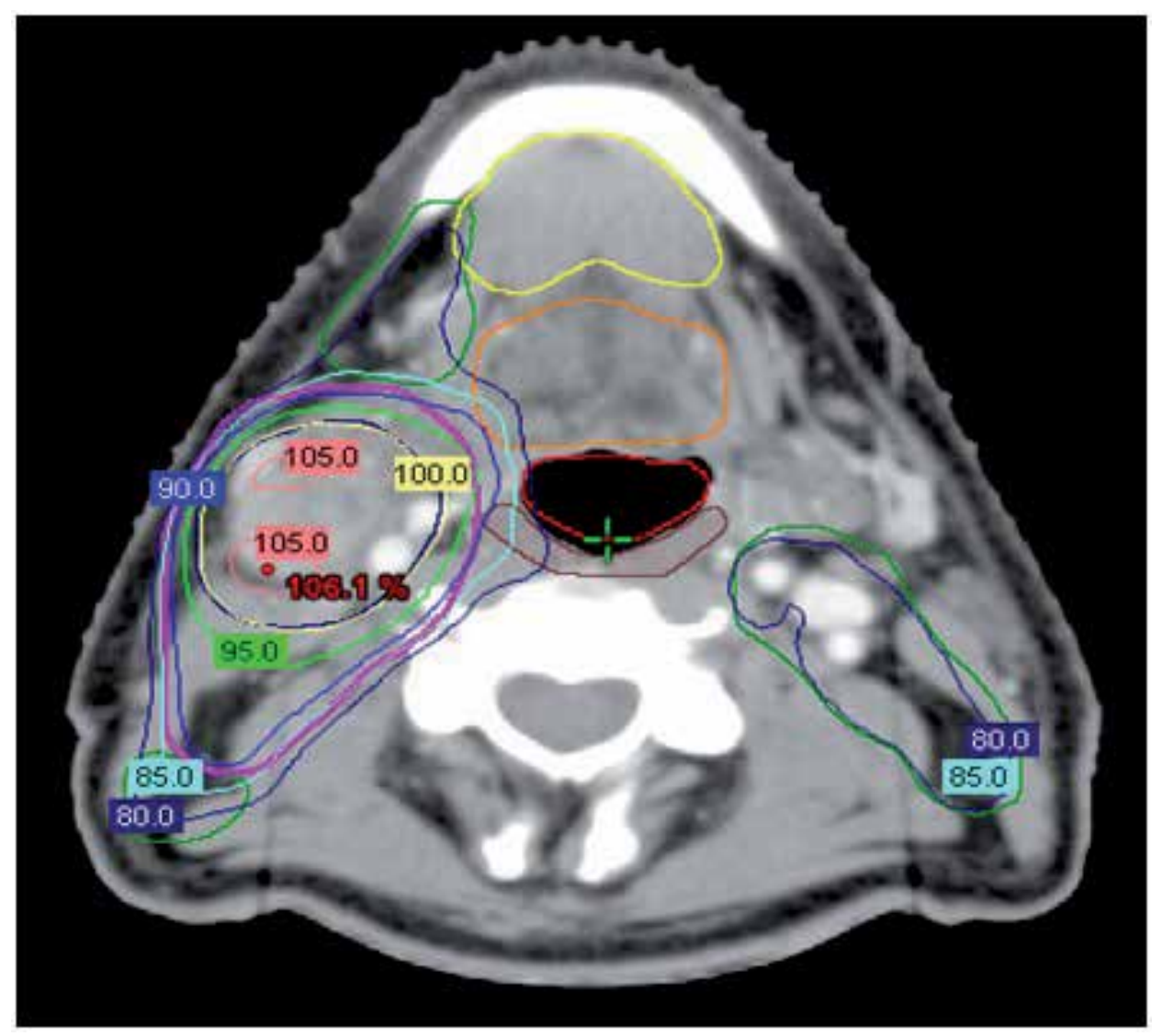

Figure 4.

Example of SWOARs-sparing IMRT plan for a patient affected by a right tonsillar cancer with omolateral adenopathies (Stage cT3N2b). Dose distributions (Gy) are labeled as percentage isodose line (coral line: 105\% isodose; yellow line: 100\% isodose; green line: $95 \%$ isodose; celestial line: $90 \%$ isodose line; cyan line: $85 \%$ isodose line; blue line: $80 \%$ isodose line). Dark green contour: low-risk target volume (54 Gy); purple contour: intermediate risk target volume (60Gy); dark blue: high risk target volume (66 Gy); brown contour: medium pharyngeal constrictor muscle; red contour: pharyngeal mucosa; orange contour: base of tongue; and yellow contour: oral cavity. 
promising results in terms of better oncologic and functional outcomes reducing the dose to the surrounding normal tissues.

Briefly, IMRT is an advanced treatment delivery technique that, exploiting the concomitant movement of multilamellar collimator during X-ray delivery through a computer-guided optimization system, produces concave isodose distributions. As a consequence, a better conformation of high treatment doses to the tumor target volume while significantly reducing the high doses to the nearest healthy tissues through a steep gradient dose is performed.

Studies on swallowing dysfunction using videofluoroscopy after radiation-based treatment revealed abnormalities in most of the previous described pharyngeal swallowing mechanisms. Hutcheson et al. [57], in those patients who referred clinical dysphagia, reported a high rate of minimal or absence of hyolaryngeal elevation and laryngeal vestibule closure, incomplete or absence of pharyngeal contraction, and minimal or no base of tongue retraction. These structures were therefore called "swallowing organs at risk (SWOARs)," and it was recommended that doses to these structures be minimized and studied to gain the potential clinical benefits and to find out the relationship between the absorbed doses to these structures and the risk of aspiration. To this aim, Christianen et al. [58] accurately defined an anatomically CT-image-based guideline for the proper delineation of these structures supposing that a RT-plan optimization to reduce doses to these structures (SWOARs-sparing IMRT) will result in better swallowing functional outcomes. An example of SWOARs-sparing IMRT plan is reported in Figure 4.

To date, despite the rationale to use IMRT to reduce posttreatment dysphagia has been well acknowledged, this assumption is still to be confirmed due to the heterogeneity of the current clinical data. Nevertheless, a positive trend seems to emerge by the literature data reporting an overall lower pattern of aspiration after IMRT compared with 3DCRT (2.6-7\% versus $7-78 \%$, respectively) and is likely to increase as the radiation oncologist will be more and more encouraged to optimize plans to SWOARs in their clinical practice [59].

To date, the greatest experience on the role of SWOARs-sparing IMRT is from the University of Michigan on 73 patients affected by locally advanced oropharyngeal cancer without the infiltration of posterior pharyngeal wall and without lateral retropharyngeal nodes [60]. In this study, authors reported the safety of dose reduction to these structures in terms of locoregional recurrence rate together with worsening of Patient-Reported Swallowing Scores (HNQOL and UWQOL questionnaires) soon after treatment (at 1 months) followed by a slow and progressive amelioration (between 6 and 12 months) and a subsequent stabilization (after 12 months).

Also, the authors reported worse swallowing scores mostly for solid rather than liquid consistencies, the lack of recovery over time with a mean VF-based SPSS score 4 (meaning necessity of modified diet requiring therapeutic intervention to minimize the risk of aspiration) as well as a pattern of aspiration between 16 and $26 \%$ (at 6 and 24 months after therapy).

The dosimetric analysis on the same patient population was subsequently performed by Eisbruch et al. [61] reporting a 50 and 25\% risk of VF-based aspiration for doses of 63 and 56 Gy to the pharyngeal constrictor muscles and for doses of 56 and $39 \mathrm{~Gy}$ to the supraglottic and glottic larynx.

These findings, despite on a smaller sample size, were confirmed by the University of Pisa mono-institutional prospective experience [62], in which 38 patients affected by naso and oropharynx cancers submitted to SWOARs-IMRT+CT were studied by combining FEES and VFS both at baseline and at 6 and 12 months. An overall moderate/severe dysphagia, based on the amount of pharyngeal residue (P-score) at FEES, accounted for 47 and $37 \%$ at 6 and 12 months for solid consistencies. Indeed, a low pattern of post-swallowing aspiration (14 and 10\% at 6 and 
12 months, respectively) was reported. Differently, videofluoroscopy (VF) findings in this studied population revealed a low pattern of post-swallowing aspiration (14 and $10 \%$ at 6 and 12 months after treatment) mostly for solid consistencies.

Interestingly, despite the lack of dosimetric correlation due to the low sample size, higher doses (median doses > 50 Gy) were delivered to the upper SWOARs (such as superior and medium constrictor muscle and base of tongue) and lower doses (median doses < $40 \mathrm{~Gy}$ ) to the inferior SWOARs.

In this regard, a different radiation-related impairment of upper and lower SWOARs, depending on the primary tumor location (i.e., naso/oropharynx versus larynx/hypopharynx), might justify a variation in the swallowing dysfunction for different consistencies of the bolus suggesting a different involvement of the single SWOARs in the deglutition act.

\section{Future directions}

Postradiation swallowing disorders is an ongoing issue that is likely to be further developed in a near future both due to its clinical relevance (mostly in the HPV era with many long-term survivor patients) and to the potential clinical benefit of modern advanced radiotherapy techniques.

At first, based on the previous findings by Pearson et al. [63], other structures to function involved in the hyolaryngeal complex elevation (the supra-hyoid musculature) are likely to be considered in the RT plan optimization process as critical organs.

In this regard, Gawryszuk et al. [64] recently integrated the SWOARs-CT image atlas with the so-called "functional swallowing units (FSUs)" involved in the hyolaryngeal elevation (floor of mouth, posterior digastric/stylohyoid complex and longitudinal pharyngeal muscles), tongue base retraction (hyoglossus and styloglossus muscle complex), and tongue motion (genioglossus and intrinsic tongue muscles) for a RT planning use.

Last but not least, despite being a discussed issue due to the current lack of evidence, the next step is likely to come out from the increasing use of proton therapy. As favorable beam properties of protons allow a higher dose conformity and thus better sparing of normal tissues without jeopardizing target dose coverage, its clinical use is likely to be translated into clinical benefits. Therefore, the SWOARs-sparing Intensity and Modulated Proton Therapy (SWOARs-IMPT) is likely to provide further advantages in terms of swallowing impairment reduction, compared with the standard SWOARs-IMRT (at least in patients with more complex clinical situations) [65].

In the meantime, we are waiting for the results from the British and Italian ongoing prospective clinical trial addressing the role of standard SWOARs-sparing IMRT (ISRCTN25458988 and NCT03448341) [66, 67].

\section{Conclusions}

Oropharyngeal dysphagia is the most frequent sequela occurring early and late after a nonsurgical RT-based treatment for HNC often leading to life threatening consequences of a "nonsafe" and/or "inefficacious swallowing act."

As such, an inefficient deglutition can cause malnutrition and dehydration with a consequent immunosuppression, immunodepression, sarcopenia, and hypovolemia-induced state. Concomitantly, an altered oropharyngeal swallowing induces a bacterial colonization in the pharyngeal tract that, in case of nonsafe deglutition causing aspiration, increases the risk to develop clinical aspiration pneumonia. 
Therefore, a proper management of radiation-related oropharyngeal dysphagia is a key issue to prevent patients from infectious-related morbidity and mortality that are reported to occur in $7-9 \%$ of patients [68].

\section{Acknowledgements}

This work was supported by grant from Lega Italiana Tumori Sezione Pisa. We would like to thank Dr. Elvio Russi, Prof. Riccardo Ruffoli, and Dr. Andrea Nacci, as experts of the issue, for their precious cultural contribution.

\section{Conflict of interest}

Authors declare no conflict of interest.

\section{Author details}

Stefano Ursino ${ }^{1 *}$, Paola Cocuzza ${ }^{1}$, Stefania Santopadre ${ }^{2}$, Fabiola Paiar $^{1}$ and Bruno Fattori ${ }^{2}$

1 Department of Radiation Oncology, University Hospital S. Chiara, Pisa, Italy

2 Otorhinolaryngology-Audiology-Phoniatric Unit, University Hospital Cisanello, Pisa, Italy

*Address all correspondence to: stefano.ursino@med.unipi.it

\section{IntechOpen}

(C) 2019 The Author(s). Licensee IntechOpen. This chapter is distributed under the terms of the Creative Commons Attribution License (http://creativecommons.org/licenses/ by/3.0), which permits unrestricted use, distribution, and reproduction in any medium, provided the original work is properly cited. (cc) BY 


\section{References}

[1] Pignon JB, Bouris J, Domenge C, et al. Chemotherapy added to locoregional treatment for head and neck squamous cell carcinoma: Three meta-analyses of updated individual data. Lancet. 2000;355:949-955. DOI: 10.1016/S0140-6736(00)90011-4

[2] Forastiere AA, Goepfert H, Maor M, et al. Concurrent chemotherapy and radiotherapy for organ preservation in advanced laryngeal cancer. The New England Journal of Medicine. 2003;349:2091-2098. DOI: 10.1056/ NEJMoa031317

[3] Delaney GP, Fisher RJ, Smee RI, et al. Split-course accelerated therapy in head and neck cancer: An analysis of toxicity. International Journal of Radiation Oncology, Biology, Physics. 1995;32:763-768. DOI: 10.1016/0360-3016(95)00093-E

[4] Nguyen TD, Panis X, Froissart D, et al. Analysis of late complications after rapid hyperfractionated radiotherapy in advanced head and neck cancers. International Journal of Radiation Oncology, Biology, Physics. 1988;14:2325. PMID: 3335458

[5] Cooper JS, Fu K, Marks J, et al. Late effects of radiation therapy in the head and neck region. International Journal of Radiation Oncology, Biology, Physics. 1995;31:1141-1164. DOI: 10.1016/0360-3016(94)00421-G

[6] Nguyen NP, Sallah S, Karlsson U, et al. Combined chemotherapy and radiation therapy for head and neck malignancies: Quality of life issues. Cancer. 2002;94:1131-1141. DOI: 10.1002/cncr.10257

[7] Nguyen NP, Moltz CC, Frank C, et al. Dysphagia following chemoradiation for locally advanced head and neck cancer. Annals of Oncology. 2004;15:383-388. DOI: 10.1093/annonc/mdh101
[8] Batth SS, Caudell JJ, Chen AM. Practical considerations in reducing swallowing dysfunction following concurrent chemoradiotherapy with intensity-modulated radiotherapy for head and neck cancer. Head and Neck. 2014;36:291-298. DOI: 10.1002/hed.23246

[9] Caudell JJ, Schaner PE, Meredith RF, et al. Factors associated with long-term dysphagia after definitive radiotherapy for locally advanced head and neck cance. International Journal of Radiation Oncology, Biology, Physics. 2009;73:410415. DOI: 10.1016/j.ijrobp.2008.04.048

[10] Raber-Durlacher JA, Brennan MT, Verdonck-de Leeuw IM, et al. Swallowing dysfunction in cancer patients. Supportive Care in Cancer. 2012;20:433-443. DOI: $10.1007 /$ s00520-011-1342-2

[11] Rosenthal DI, Lewin JS, Eisbruch A. Prevention and treatment of dysphagia and aspiration after chemoradiation for head and neck cancer. Journal of Clinical Oncology. 2006;24:2636-2643. DOI: 10.1200/ JCO.2006.06.0079

[12] Forastiere AA, Zhang Q, Weber RS, et al. Long-term results of RTOG 91-11: A comparison of three nonsurgical treatment strategies to preserve the larynx in patients with locally advanced larynx cancer. Journal of Clinical Oncology. 2013;31(7):845-852. DOI: 10.1200/JCO.2012.43.6097

[13] Robbins KT. Barriers to winning the battle with head and neck cancer. International Journal of Radiation Oncology, Biology, Physics. 2002;53(1):4-5. PMID: 12007934

[14] Jean A. Brain stem control of swallowing: Neuronal network and cellular mechanisms. Physiological Reviews. 2001;81:929-969. DOI: 10.1152/ physrev.2001.81.2.929 
[15] Rofes L, Cola P, Clavé P. The effects of sensory stimulation on neurogenic oropharyngeal dysphagia. Journal of Gastroenterology and Hepatology Research. 2014;3:1066-1072. DOI: 10.1136/gutjnl-2011-300753

[16] Alvarez-Berdugo D, Rofes L, Casamitjana JF, Padrón A, Quer M, Clavé P. Oropharyngeal and laryngeal sensory innervation in the pathophysiology of swallowing disorders and sensory stimulation. Annals of the New York Academy of Sciences. 2016;1380(1):104-120

[17] Logemann JA. Evaluation and Treatment of Swallowing Disorders. Austin: Pro-ed, An International Publisher; 1998. p. 5-47; 281-298

[18] Palmer JB, Tanaka E, Siebens AA. Motion of the posterior pharyngeal in swallowing. Laryngoscope. 1988;98:414-417. DOI: 10.1288/00005537-198804000-00012

[19] O’Connor A, McConnel FMS. Dysphagia associated with pharyngoesophageal segment dysfunction. Acta Oto-RhinoLaryngologica Belgica. 1994;104: 1376-1382. PMID: 8209678

[20] BourguignatE, EvennouA, ButinAM, Bobin S. Insuffisance velopharyngéer. In: EMC Otorhinolaryngology. Paris: Elsevier; 1996. p. 20-618-A-10. DOI: 10.1016/S0246-0351(05)39253-1

[21] Ohmae Y, Logeman JA, Kaiser P. Timing of glottic closure during normal swallow. Head \& Neck. 1995;17:394-402. PMID: 8522440

[22] Martin-Harris B, Brodsky MB, Michel Y, et al. MBS measurement tool for swallow impairment-MBSImp: Establishing a standard. Dysphagia. 2008;23:392-405. DOI: $10.1007 /$ s00455-008-9185-9

[23] Murray J. Manual of Dysphagia Assessment in Adults. Dysphagia Series.
New York: Delmar Cengage Learning; 1999. pp. 90-143

[24] Pearson WG Jr, Taylor BK, Blair J, Martin-Harris B. Computational analysis of swallowing mechanics underlying impaired epiglottic inversion. Laryngoscope. 2016;126:1854-1858. DOI: 10.1002/ lary. 25788

[25] Pearson WG Jr, Davidoff AA, Smith ZM, Adams DE, Langmore SE. Impaired swallowing mechanics of post radiation therapy head and neck cancer patients: A retrospective videofluoroscopic study. World Journal of Radiology. 2016;8:192-199. DOI: 10.4329/wjr.v8.i2.192

[26] Pearson WG Jr, Langmore SE, Zumwalt AC. Evaluating the structural properties of suprahyoid muscles and their potential for moving the hyoid. Dysphagia. 2011;26:345-351. DOI: 10.1007/s00455-010-9315-z

[27] Krespi YP, Blitzer A, editors. Aspiration and swallowing disorders. Otolaryngologic Clinics of North America. 1988;4:21

[28] Jacob P, Kahrilas PJ, Logemann JA, Shah V, Ha T. Upper esophageal sphincter opening and modulation during swallowing. Gastroenterology. 1989;97:1469-1478. PMID: 2583413

[29] Mu L, Sanders I. Neuromuscular compartments and fiber-type regionalization in the human inferior pharyngeal constrictor muscle. The Anatomical Record. 2001;264:367-377. DOI: 10.1002/ar.10020

[30] Alvarez-Berdugo D, Rofes L, Casamitjana JF, Padròn A, Quer M, Clavè $P$. Oropharyngeal and laryngeal sensory innervation in the pathophysiology of swallowing disorders and sensory stimulation treatments. Annals of the New York 
Academy of Sciences. 2016;1380: 1104-1120. DOI: $10.1111 /$ nyas.13150

[31] Mu L, Sanders I. Neuromuscolar specializations within human pharyngeal constrictor muscles. The Annals of Otology, Rhinology, and Laryngology. 2007;116(8):604-617. DOI: $10.1177 / 000348940711600809$

[32] Mu L, Sanders I. Muscle fiber-type distribution pattern in the human cricopharyngeus muscle. Dysphagia. 2002;17:87-96. DOI: $10.1007 /$ s00455-001-0108-2

[33] Venker-van Haagen AJ, Hartman W, Wolvekamp WTC. Contributions of the glossopharyngeal nerve and the pharyngeal branch of the vagus nerve to the swallowing process in dogs. American Journal of Veterinary Research. 1986;47:1300-1307. PMID: 3729131

[34] Lazarus CL, Logemann JA, Pauloski BR, Colangelo LA, Kahrilas PJ, Mittal BB, et al. Swallowing disorders in head and neck cancer patients treated with radiotherapy and adjuvant chemotherapy. Laryngoscope. 1996;106(9 Pt 1):1157-1166. PMID: 8822723

[35] Anderson EJ, Neufer PD. Type II skeletal myofibers possess unique properties that potentiate mitochondrial $\mathrm{H}(2) \mathrm{O}(2)$ generation. American Journal of Physiology. Cell Physiology. 2006;290(3):C844-C851. DOI: 10.1152/ ajpcell.00402.2005

[36] Viet CT, Corby PM, Akinwande A, Schmidt BL, Viet CT, Corby PM, et al. Review of preclinical studies on treatment of mucositis and associated pain. Journal of Dental Research. 2014;93(9):868-875. DOI: $10.1177 / 0022034514540174$

[37] Simonyan K, Feng X, HenriquezVM, Ludlow CL. Combined laryngeal inflammation and trauma mediate long-lasting immunoreactivity response in the brainstem sensory nuclei in the rat. Frontiers in Integrative Neuroscience. 2012;6:97. DOI: $10.3389 /$ fnint.2012.0

[38] Gillette EL, Mahler PA, Powers BE, Gillette SM, Vujaskovic Z. Late radiation injury to muscle and peripheral nerves. International Journal of Radiation Oncology, Biology, Physics. 1995;31(5):1309-1318. DOI: 10.1016/0360-3016(94)00422-h

[39] Rong X, Tang Y, Chen M, Lu K, Peng Y. Radiation-induced cranial neuropathy in patients with nasopharyngeal carcinoma. A follow-up study. Strahlentherapie und Onkologie. 2012;188(3):282-286. DOI: $10.1007 /$ s00066-011-0047-2

[40] King SN, Dunlap NE, Tennant PA, Pitts T. Pathophysiology of radiationinduced dysphagia in head and neck cancer. Dysphagia. 2016;31(3):339-351. DOI: $10.1007 / \mathrm{s} 00455-016-9710-1$

[41] Schadrach JL, Wagers AJ. Stem cells for skeletal muscle repair. Philosophical Transactions of the Royal Society. 2011;366:2297-2306. DOI: 10.1098/ rstb.2011.0027

[42] Shefer G, Rauner G, Reuveni Yablonka Z, Benayhau D. Reduced satellite cell numbers and myogenic capacity in aging can be alleviated by endurance exercise. PLoS One. 2010;5(10):e13307. DOI: 10.1371/journal. pone. 0013307

[43] Shefer G, Carmeli E, Rauner G, Yablonka-Reuveni Z, Benayahu D. Exercise running and tetracycline as means to enhance skeletal muscle stemcell performance after external fixation. Journal of Cellular Physiology. 2008;215(1):265-275. DOI: $10.1002 /$ jcp.21306

[44] De Lisio M, Kaczor JJ, Phan N, Tarnopolsky MA, Boreham DR, Parise G. Exercise training enhances the 
skeletal muscle response to radiationinduced oxidative stress. Muscle \& Nerve. 2011;43(1):58-64. DOI: 10.1002/ mus. 21797

[45] Hood DA. Invited review: Contractile activity-induced mitochondrial biogenesis in skeletal muscle. Journal of Applied Physiology (Bethesda, MD: 1985). 2001;90(3):1137-1157. DOI: 10.1152/jappl.2001.90.3.1137

[46] Adhihetty PJ, Irrcher I, Joseph AM, Ljubicic V, Hood DA. Plasticity of skeletal muscle mitochondria in response to contractile activity.

Experimental Physiology. 2003;88(1):99-107. PMID: 12525859

[47] Scheele C, Nielsen S, Pedersen BK. ROS and myokines promote muscle adaptation to exercise. Trends in Endocrinology and Metabolism: TEM. 2009;20(3):95-99. DOI: 10.1016/j. tem.2008.12.002

[48] Venditti P, Masullo P, Di Meo S. Effect of training on $\mathrm{H}(2) \mathrm{O}(2)$ release by mitochondria from rat skeletal muscle. Archives of Biochemistry and Biophysics. 1999;372(2):315-320. DOI: 10.1006/abbi.1999.1494

[49] Phillips SM, Glover EI, Rennie MJ. Alterations of protein turnover underlying disuse atrophy in human skeletal muscle. Journal of Applied Physiology (Bethesda, MD: 1985). 2009;107(3):645-654. DOI: 10.1152/japplphysiol.00452.2009

[50] Glover EI, Phillips SM, Oates BR, Tang JE, Tarnopolsky MA, Selby A, et al. Immobilization induces anabolic resistance in human myofibrillar protein synthesis with low and high dose amino acid infusion. The Journal of Physiology. 2008;586(Pt 24):6049-6061. DOI: 10.1113/jphysiol.2008.160333

[51] Paddon-Jones D, Sheffield-Moore M, Cree MG, Hewlings SJ, Aarsland A, Wolfe RR, et al. Atrophy and impaired muscle protein synthesis during prolonged inactivity and stress. The Journal of Clinical Endocrinology \& Metabolism. 2006;91(12):4836-4841. DOI: $10.1210 /$ jc.2006-0651

\section{[52] Krisciunas GP, Sokoloff W,} Stepas K, Langmore SE. Survey of usual practice: Dysphagia therapy in head and neck cancer patients. Dysphagia. 2012;27(4):538-549. DOI: $10.1007 /$ s00455-012-9404-2

[53] Kraaijenga SA, van der Molen L, Jacobi I, Hamming-Vrieze O, Hilgers FJ, van den Brekel MW. Prospective clinical study on long-term swallowing function and voice quality in advanced head and neck cancer patients treated with concurrent chemoradiotherapy and preventive swallowing exercises. European Archives of Oto-RhinoLaryngology. 2015;272(11):3521-3531. DOI: $10.1007 / \mathrm{s} 00405-014-3379-6$

[54] Carnaby-Mann G, Crary MA, Schmalfuss I, Amdur R. "Pharyngocise": Randomized controlled trial of preventative exercises to maintain muscle structure and swallowing function during head-and-neck chemoradiotherapy. International Journal of Radiation Oncology, Biology, Physics. 2012;83(1):210-219. DOI: 10.1016/j.ijrobp.2011.06.1954

[55] Hutcheson KA, Bhayani MK, Beadle BM, Gold KA, Shinn EH, Lai SY, et al. Eat and exercise during radiotherapy or chemoradiotherapy for pharyngeal cancers: Use it or lose it. JAMA Otolaryngology. Head \& Neck Surgery. 2013;139(11):1127-1134. DOI: 10.1001/jamaoto.2013.4715

[56] Ursino S, Seccia V, Cocuzza P, et al. How does radiotherapy impact swallowing function in nasopharynx and oropharynx cancer? Shortterm results of a prospective study. Acta Otorhinolaryngologica Italica. 2016;36:174-184. DOI: 10.14639/0392-100X-640 
[57] Hutcheson KA, Lewin JS, Barringer DA, Lisec A, Gunn GB, Moore $\mathrm{M}$, et al. Late dysphagia after radiotherapy-based treatment of head and neck cancer. Cancer. 2012;118(23):57935799. DOI: $10.1002 /$ cncr.27631

[58] Christianen M, Langendijk JA, Westerlaan HE, van de Water TA, Bijl HP. Delineation of organs at risk involved in swallowing for radiotherapy treatment planning. Radiotherapy and Oncology. 2011;101:394-402

[59] Ursino S, D’Angelo E, Mazzola R, Merlotti A, Morganti R, Cristaudo A, et al. A comparison of swallowing dysfunction after three-dimensional conformal and intensity-modulated radiotherapy: A systematic review by the Italian Head and Neck Radiotherapy Study Group. Strahlentherapie und Onkologie. 2017;193(11):877-889. DOI: 10.1007/s00066-017-1160-7

[60] Feng FY, Kim HM, Lyden TH, Haxer MJ, Worden FP, Feng M, et al. Intensity-modulated chemoradiotherapy aiming to reducedysphagia in patients with oropharyngeal cancer: Clinical and functional results. Journal of Clinical Oncology. 2010;28:2732-2738. DOI: 10.1200/JCO.2009.24.6199

[61] Eisbruch A, Kim HM, Feng FY, Lyden TH, Haxer MJ, Feng M, et al. Chemo-IMRT of oropharyngeal cancer aiming to reduce dysphagia: Swallowing organs late complication probabilities and dosimetric correlates. International Journal of Radiation Oncology, Biology, Physics. 2011;81:e93-e99. DOI: 10.1016/j. ijrobp.2010.12.067

\section{[62] Ursino S, Cocuzza P, Seccia V,} DelishajD, CristaudoA, PasqualettiF, etal. Pattern of dysphagia after swallowingsparing intensity-modulated radiotherapy (IMRT) of head and neck cancers: Results of a mono-institutional prospective study. Strahlentherapie und Onkologie. 2018;194(12):1114-1123. DOI: $10.1007 / \mathrm{s} 00066-018-1328-9$
[63] Pearson W, Hindson DF,

Langmore SE, Zumwalt AC. Evaluating swallowing muscles essential for hyolaryngeal elevation by using muscle functional magnetic resonance imaging. International Journal of Radiation Oncology, Biology, Physics. 2013;85(3):735-740. DOI: 10.1016/j. ijrobp.2012.07

[64] Gawryszuk A, Bijl HP, Holwerda M, Halmos GB, Wedman J, Witjes MJH, et al. Functional swallowing units (FSUs) as organs-at-risk for radiotherapy. Part 2: Advanced delineation guidelines for FSUs. Radiotherapy and Oncology. 2019;130:6874. DOI: 10.1016/j.radonc.2018.09.022

[65] Langendijk JA, Boersma LJ, Rasch CRN, van Vulpen M, Reitsma JB, van der Schaaf $A$, et al. Clinical trial strategies to compare protons with photons. Seminars in Radiation Oncology. 2018;28(2):79-87. DOI: 10.1016/j.semradonc.2017.11.008

[66] Petkar I, Rooney K, Roe JW, Patterson JM, Bernstein D, Tyler JM, et al. DARS: A phase III randomised multicentre study of dysphagiaoptimised intensity-modulated radiotherapy (Do-IMRT) versus standard intensity-modulated radiotherapy (S-IMRT) in head and neck cancer. BMC Cancer. 2016;16(1):770. DOI: $10.1186 / \mathrm{s} 12885-016-2813-0$

[67] Clinical and instrumental evaluation of late dysphagia in patients with oropharynx and nasopharynx cancer, candidates to combined radiochemotherapy with intensity modulated radiotherapy (IMRT). Available from: https://clinicaltrials.gov/ ct2/show/NCT03448341

[68] Mirabile A, Numico G, Russi EG, Bossi P, Crippa F, Bacigalupo A, et al. Sepsis in head and neck cancer patients treated with chemotherapy and radiation: Literature review and consensus. Critical Reviews in Oncology/ Hematology. 2015;95(2):191-213. DOI: 10.1016/j.critrevonc.2015.03.003 
Section 6

Salivation by

Somatosensory

Stimulation 



\title{
Effect of Salivation by Facial Somatosensory Stimuli of Facial Massage and Vibrotactile Apparatus
}

\author{
Tsunoda Yumi, Akatuka Sumiko, Fukui Sayaka, \\ Nakayama Enri, Abe Kimiko, Sato Mituyasu, \\ Kimura Masanori, Kato Syunnichiryou, Sakai Maho, \\ Yamaoka Masaru, Watanabe Mao, Ueda Koichirou \\ and Hiraba Hisao
}

\begin{abstract}
We studied the effects of salivary promotion of fluid secretion after hand massage, and the apparatus of vibrotactile stimulation ( $89 \mathrm{~Hz}$ frequency, $15 \mathrm{~min}$ ) in normal humans. Personal massage cannot be performed on handicap and stroke patients, and then giving hand massage to them for $5 \mathrm{~min}$ massage gives a tired feeling. So, we focused 3 min stranger massage. Salivary glands can discharge the accumulated saliva by extrusion from the acinus glands' massages as described in the recent Japanese textbook. We think that this method may not produce realistic recovery. Our aim ideas are to relieve stress and increase temperature with lightly touch massage of the skin and for a 1 cycle of $1 \mathrm{~s}$. We recorded RR interval of ECG, total salivation, facial skin temperature, $\mathrm{OxyHb}$ of fNIRS on the frontal cortex, and amylase activity for the autonomic changes. In increased $2^{\circ} \mathrm{C}$ of the facial skin temperature, the hand massage had a need for $3 \mathrm{~min}$ and the vibrotactile stimulation for $15 \mathrm{~min}$. Increase from 700 to $1000 \mathrm{~ms}$ of RR intervals had a need for $3 \mathrm{~min}$ in the hand massage and had $15 \mathrm{~min}$ in the vibrotactile stimulation. Although vibrotactile stimulation needs long time of 4-7 years as effective recovery, hand massage may have more effect with a repetition of day after day.
\end{abstract}

Keywords: hand massages, vibrotactile stimulation, facial skin temperature, total salivation, RR intervals of ECG, OxyHb of fNIRS, amylase activity

\section{Introduction}

Does somatosensory stimulation in areas of the face and oral cavity promote salivation and recovery of poor salivary glands? We studied the effects of salivary promotion after hand massage and apparatus of vibrotactile stimulation $(89 \mathrm{~Hz}$ frequency, $9.8 \mu \mathrm{m}$ amplitude, and $15 \mathrm{~min}$ ) in normal humans [1]. Namely, we think that the produce of salivation needs effective changes of an autonomic 
system for salivation following autonomic activity. Quality of saliva is transmuted by autonomic activity of sympathetic or parasympathetic nerve. In sympathetic activity, viscid saliva functions to allow a food bolus to be easily passed from the mouth into the esophagus. Furthermore, in parasympathetic activity, it is also very important function for the sense of taste and for digestion. In particular, as the poor salivation cannot make the food bolus, dysphasia troubles are induced. On the other hand, poor salivation leads to an increase in dental caries and gingivitis. For ill-fitting dentures of deficits of oral function, massage therapists of the salivation need a rehabilitation method for encouraging salivation and orofacial function [2]. Namely, poor salivations following sickness or advanced aging may be recovered by activation of metabolism of salivary glands. We know that the method of facial traditional massage can induce salivation with increased temperature and blood flow by directly stimulating the salivary glands. However, the descriptions of salivary glands massage from the recent Japanese text will generate salivation by extrusion of accumulated salivary from the acinus glands, and approximately totally 10 pushes form at the anterior to posterior regions of the parotid glands. Furthermore, in the textbook, the submandibular gland is pressed about five times by the thumb to grip the soft parts inside the angle of the mandible, and the sublingual gland is pressed about five times by the thumb from under the chin [3]. The traditional method of saliva massages involved pressing the acinar regions depending on saliva accumulated in that region. However, the principal goal of massages is to maintain one's metabolism. Thus, we think that the real massage must maintain the metabolism of salivary glands at high levels depending on increasing the temperature of facial skin via haptic stimuli, a paratripsis, through the use of the palms of the hand [4]. The real salivary gland massages must be performed to activate the acinar regions. Functional recovery of salivation is encouraged by promotion of the metabolism of the salivary glands, and a rise in metabolism produces with increasing blood circulation in the salivary glands. This increase in circulation also elevates the temperature around the gland which may activate acinus gland cells. Initially, we examined the effect of facial somatosensory stimuli by using of the apparatus of vibrotactile stimuli $[1,5-8]$. Secondly, we tried to carry out hand massages, again. So, we will report the effects of salivation in facial vibrotactile stimuli and facial hand massages.

\section{Materials and methods}

The Nihon University School of Dentistry provides ethical approval to conduct this pilot clinical study (approval number: 2009-5). All study participants received verbal explanations regarding measurements of hand massages and a vibrotactile apparatus and signed an informed consent. The study participants were again explained about the protection of privacy and personal information and provided the freedom to continue or withdraw their consent.

\subsection{The optimum way of hand massage}

We performed facial stranger or personal hand stimuli during 3 and $5 \mathrm{~min}$. After performing the stranger or self-massage procedures for 3 or $5 \mathrm{~min}$, we asked for degree of fatigue. We included 40 healthy subjects (age 26.7 $\pm 2.4 ; 25$ males, 15 females) and examined well-suited massage time and method depending on first $5 \mathrm{~min}$ and then $3 \mathrm{~min}$ massage. However, normal subjects were always appealed for feeling of fatigue with an exercise for $5 \mathrm{~min}$. Hand massage for $3 \mathrm{~min}$ can be divided 
into stranger and self-massages: own face has been massaged by other person' hand or by own hand. Personal massage is not able to treat patients with handicap and cerebral apoplexy. So, we focused at the exercise for 3 minutes and stranger massage (hand massage), as shown in Figure 1A.

After a stranger massage (a light touch and paratripsis massage) of the facial skin, we performed a rotation every second to increase the temperature of the skin and thus boost local blood circulation and improve the metabolism of the parotid glands and around the facial skin (Figure 1B).

We performed the resting and stimulating phases during insertion of cotton rolls for each 3 min between 5 and $7 \mathrm{pm}$ under the circadian rhythm. We explored ECG (electrocardiogram), facial skin temperature, total salivations, OxyHb (oxidation hemoglobin) activity of f-NIRS (functional near-infrared spectroscopy) on the frontal cortex and amylase activity between resting and massage phase of the stranger hand massage. We recorded measurement of resting condition for $3 \mathrm{~min}$ after each cotton roll was set into the oral cavity, and then after relax of $1 \mathrm{~min}$ interval, new cotton roll was fitted into an oral cavity again and we did measurement of stimulating condition for $3 \mathrm{~min}$. The facial skin temperature, RR intervals of ECG and f-NIRS recorded during experiments, and the observational study were measured in each measuring range. Furthermore, we recorded total salivation and saliva amylase activity after each phase of resting, hand massage, and vibrotactile stimulation.

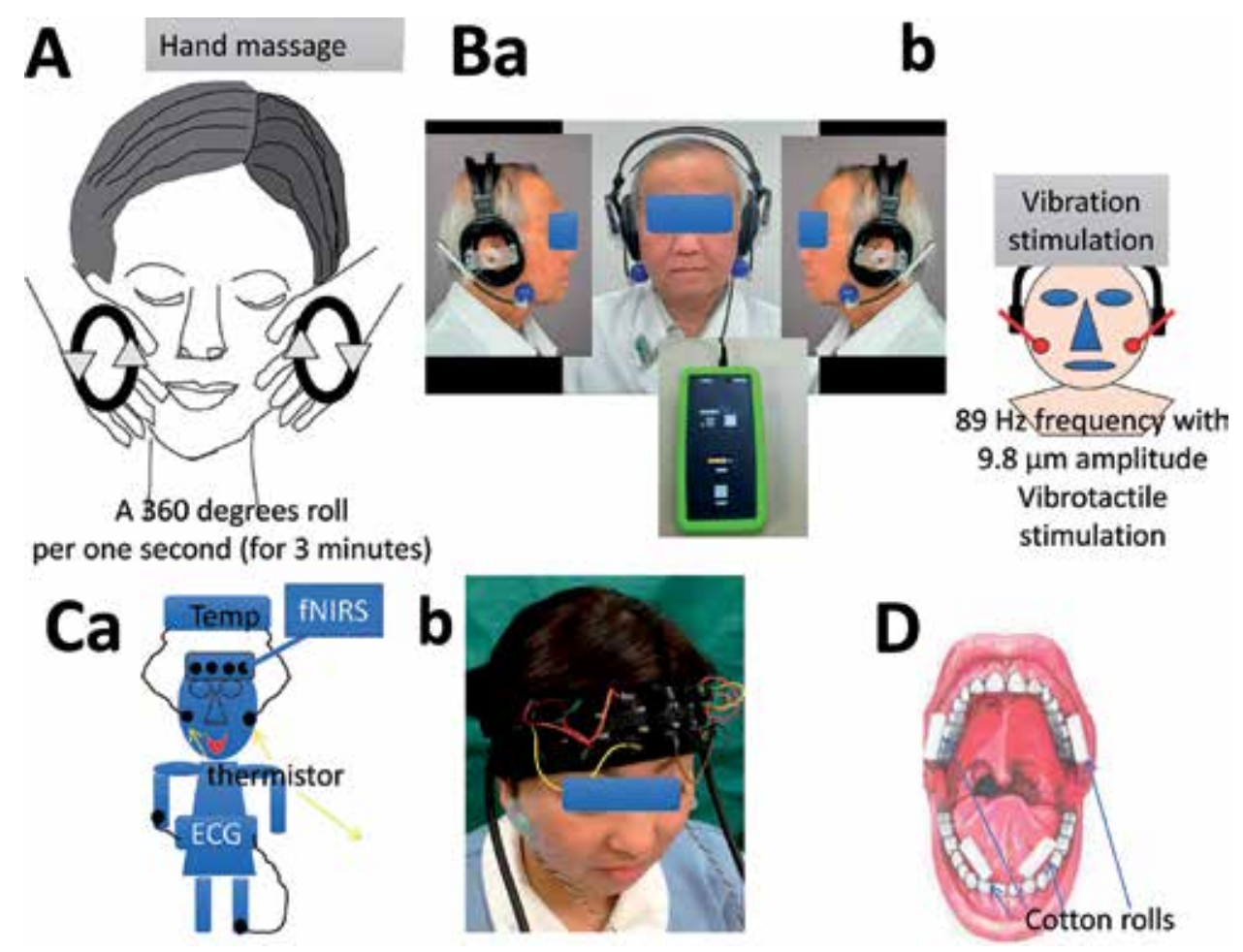

Figure 1.

Experimental method. (A) Stranger hand massages performed at $360^{\circ}$ roll per 1 s for 3 min. (B-a and $-b$ ) $89 \mathrm{~Hz}$ and $9.8 \mu \mathrm{m}$ amplitude vibrotactile stimulation. (C-a) Thermometers on the facial skin, electrodes of electrocardiogram (ECG), and electrical poles of functional near-infrared spectroscopy (fNIRS) in the frontal cortex and the position of electrodes of ECG. (C-b) Photograph of electrical poles of $f$ NIRS and thermistors. (D) The position of cotton rolls into the intraoral cavity. Cotton rolls under the tongue were recorded salivations of the submandibular and lingual glands and cotton rolls on the buccal mucosa of the upper jaw were recorded salivations of the parotid glands. 


\subsection{The optimum frequency of vibrotactile apparatus}

Apparatus of $89 \mathrm{~Hz}$ frequency and $9.8 \mu \mathrm{m}$ amplitude vibrotactile stimulation was chosen by data of the previous papers [1, 5-8], as shown in Figure 1B-a, -b. We tried three vibrotactile stimuli, $(89,114$, and $180 \mathrm{~Hz}$, and others all of $9.8 \mu \mathrm{m}$ amplitude), and the best salivation was $89 \mathrm{~Hz}$ frequency in comparison with others, as shown in Figure 2. This apparatus was used by poor salivation patients (especially, Sjogrens's syndrome and so on) and about $50 \%$ of patients showed the effect [1]. Stranger hand massage had a limit time to do massage for physical fatigue, so we tried the $89 \mathrm{~Hz}$ frequency vibrotactile apparatus for long time of facial stimulation. Furthermore, we recorded facial skin temperature, total salivation, RR intervals of ECG, and saliva amylase activity, too.

We explored the facial skin temperature, ECG recording, and fNIRS on the frontal cortex for the comparison with resting for $3 \mathrm{~min}$, stranger massage for $3 \mathrm{~min}$, and vibrotactile apparatus for $15 \mathrm{~min}$.

\subsection{Analysis of facial skin temperature}

Facial skin temperature was measured by thermistor-pots (BioResarch Co.) located on the facial skin of parotid glands of both sides with adhesive tape, as shown in Figure 1C-a, - b. We recorded the temperature through the experiment and analyzed $3 \mathrm{~min}$ from start to finish during resting phase, 3 min from start to finish during stranger hand massage, and $15 \mathrm{~min}$ from start to finish during the vibrotactile stimulation.

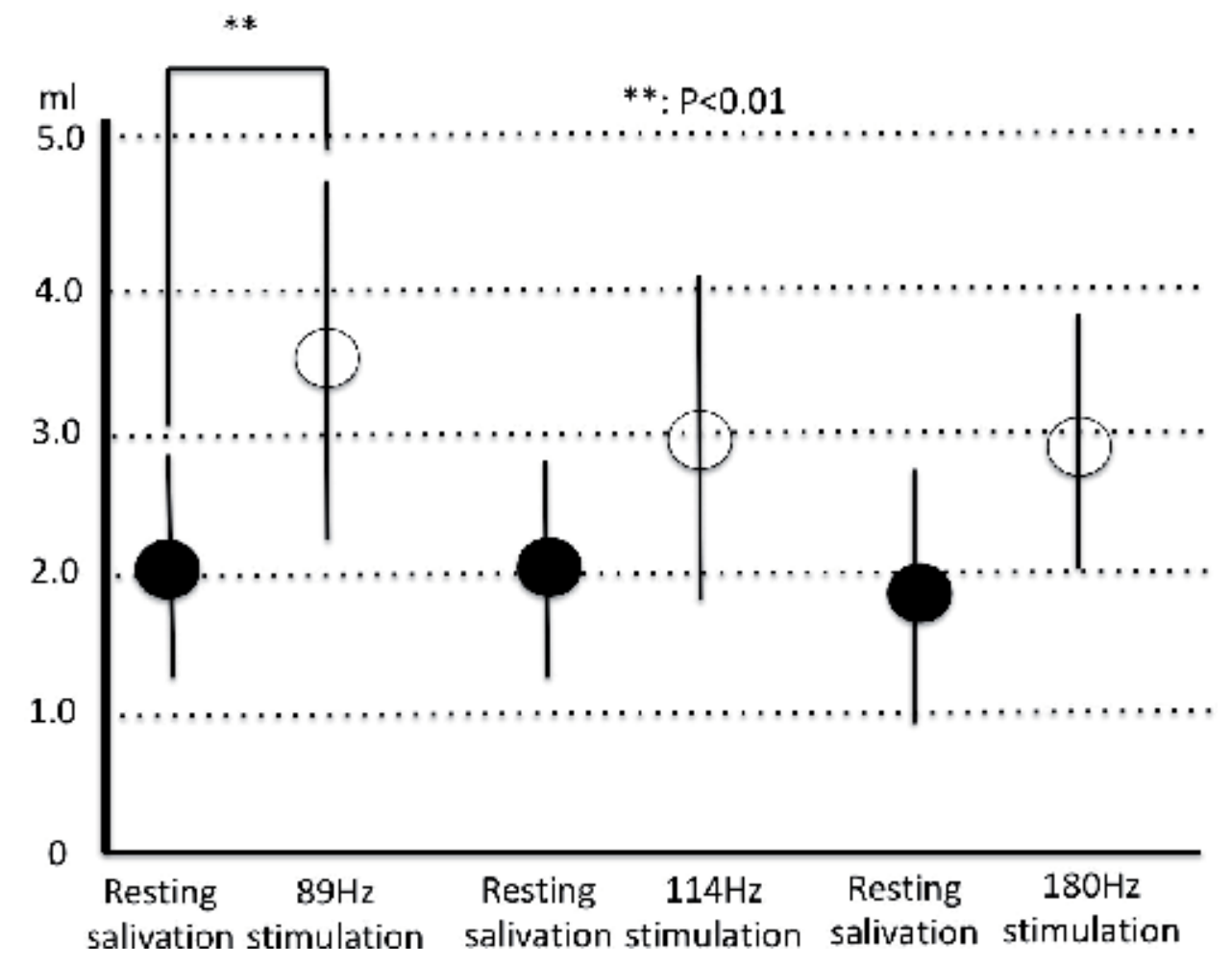

Figure 2.

Total salivation in resting salivation and vibrotactile stimuli $(89,114$, and $180 \mathrm{~Hz}$ frequency). $p<0.01, t$-test. 


\subsection{Analysis of RR intervals of ECG}

The electrocardiogram (ECG) recording was induced by the standard limb lead. The heart rate was measured by the RR interval rate (time between $R$ and $\mathrm{R}$ ) and was analyzed by the variance plot and frequency distribution, as shown in Figure 1C-a. On the other hand, in the distribution of frequency measured by RR interval rate, we decided a mean RR interval value in order to show almost monomodal peaks [9].

\subsection{Analysis of total salivation}

The mouths of each subject were fitted with rolls of cotton placed on both sides of the mouth at the following sites on the duct openings of the parotid glands of the buccal mucosa near the second upper molar teeth, and under the tongue to collect saliva from the sublingual and submandibular glands for a measurement of total salivation, as shown in Figure 1D. The total salivations indicate the sum of four cotton rolls (both sides of parotid, and submandibular and sublingual glands), as shown in Figure 1D. We measured the resting salivation for $3 \mathrm{~min}$ and after a relaxing interval of a minute, we did stranger hand massages salivation for $3 \mathrm{~min}$ again. Furthermore, we measured total salivations of the $15 \mathrm{~min}$ vibrotactile stimulation after $5 \mathrm{~min}$ resting interval of the massage.

\subsection{Analysis of an OxyHb of fNIRS on the frontal cortex}

The recording was conducted using a functional near-infrared spectroscopy (fNIRS) OEG16 instrument (SpectraTech, Inc., Shelton, CT, USA) from the frontal cortex. As shown in Figure 1C-a, -b, the fNIRS probe assembly consisted of six LEDs as light sources, each of which emitted two kind of wavelengths, 770 and $840 \mathrm{~nm}$, and six photodiodes as detectors. The sources and detectors were symmetrically arranged in an area of 3.0-14.0 cm, with the nearest source-detector separation of $2.0 \mathrm{~cm}$, and measurement points were at 16 points on a frontal cortex. During scanning, a velcro band held the probe assembly securely to the forehead of subjects and extended from ear to ear horizontally and from hairline to eyebrows vertically. Each of the LEDs was turned on in sequence, and the diffuse NIR light from each source was acquired through the cortical region at the nearest detector. Thus, 16 source-detector pairs (channels) in total were measured (Figure 1C-a, -b). The sampling rate across all 16 channels was $0.76 \mathrm{~Hz}$. In particular, we showed a 16-channel computerized analysis (as shown in the previous papers [5-7]) and the original waves of four channel recording areas in the central parts. Analysis of amount of $\mathrm{OxyHb}$ of fNIRS on the frontal cortex is examined by the program of fNIRS Data Viewer, and we explored channels 4, 7, 10, and 13 of the central part of the frontal cortex. Data recorded by the experiment measured the value of integral of four channels in the center frontal cortex, and we calculated mean value of integral of four waves $[5,6,10,11]$.

\subsection{Analysis of salivary amylase activity}

A salivary amylase ( $\alpha$-amylase) activity was measured by salivary amylase monitor (Nipro Co.) provided for chips of salivary amylase monitor. Measurements of saliva amylase activities were recorded after resting, stranger hand massage, and vibrotactile stimulation phases. 


\section{Results}

We examined the effect of salivation in resting and stimulating stages between the stranger hand massage and vibrotactile apparatus. We focused on a $3 \mathrm{~min}$ stranger massage, because a self-massage could not be performed for treating patients with handicap and stroke, and 5 min massage evoked the tired feeling.

\subsection{Facial skin temperature}

In 5 min hand massages, facial skin temperature increased about $2-3^{\circ} \mathrm{C}$ in both hand massages (stranger and personal). On the other hand, in $3 \mathrm{~min}$ stimuli, facial skin temperature increased about $1.5-2.0^{\circ} \mathrm{C}$. The tendency of increased facial skin temperature in 3 and 5 min stimuli was almost same; namely, the ratio of increased temperature was dependent on stimulus time. On the other hand, in performance of stranger hand massages for $5 \mathrm{~min}$, each operator complained with feeling of fatigue, and sometimes showed overflowing of salivation in the cotton rolls of the oral cavity. From this result, we abandoned this experiment of $5 \mathrm{~min}$ stimulation depending on using of five subjects. Furthermore, the 5 min massage procedure elicited the sure fatigue. So, we focused to 3 min stranger hand massage.

In loading, facial skin temperature showed about $33^{\circ} \mathrm{C}$ in the insert of cotton rolls in an oral cavity, as shown in Figure 3. This skin temperature may be stimulated by a foreign substance, because facial skin temperature was decreased up to about $32^{\circ} \mathrm{C}$ in the resting phase after loading. After a resting phase of $3 \mathrm{~min}$ stranger massage stage, facial skin temperature in the stranger massage increased from 32.0 to $34.5^{\circ} \mathrm{C}$, as shown in Figure 3; namely, stranger massage showed the increase of facial skin temperature from 32.0 to 34.5 in $3 \mathrm{~min}$ stimulation.

On the other hand, the apparatus of $89 \mathrm{~Hz}$ frequency and $9.8 \mu \mathrm{m}$ amplitude vibrotactile stimulation was chosen by data of the previous papers $[1,5-8]$, as shown in Figure 1. This vibrotactile stimulation was the best salivation in comparison with others, as shown in Figure 2. In the resting phase, facial skin temperature showed about $32^{\circ} \mathrm{C}$. Furthermore, the start of $89 \mathrm{~Hz}$ vibrotactile stimulation showed about $33^{\circ} \mathrm{C}$ and after $15 \mathrm{~min}$, it did $34^{\circ} \mathrm{C}$; namely, an increase of $1^{\circ} \mathrm{C}$ spent due to 15 min vibrotactile stimulation, as shown in Figure 3.

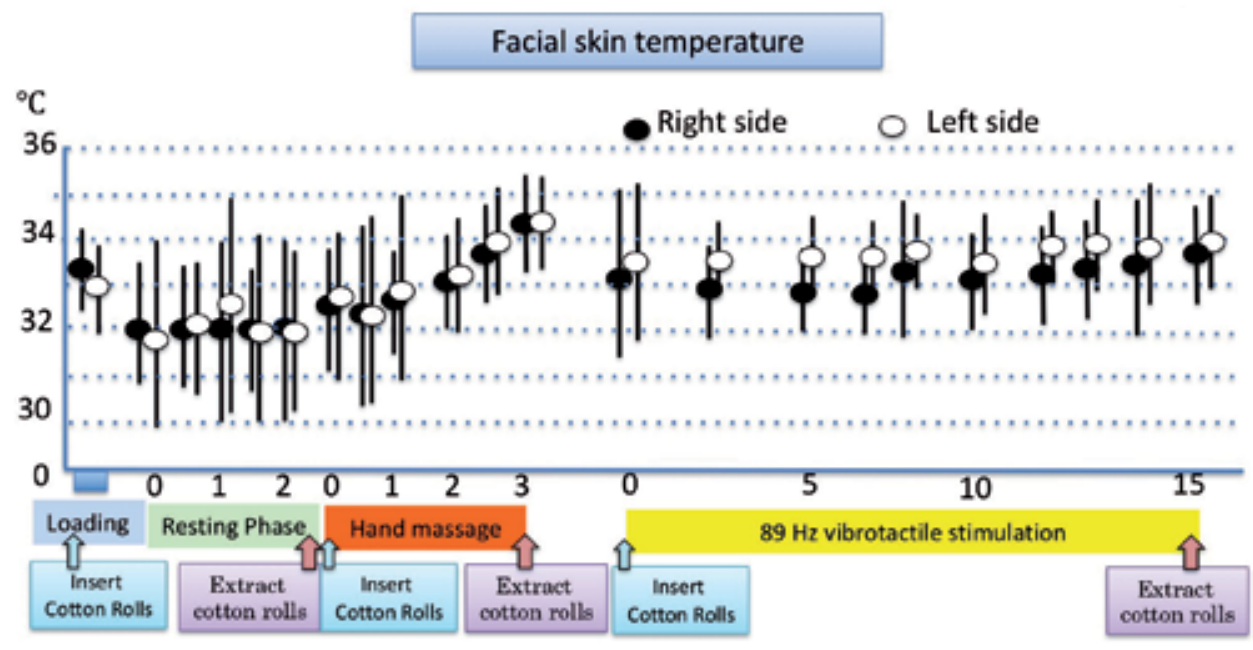

Figure 3.

Changes in temperatures on facial skin before and after 3 min resting phase, 3 min stranger massage, and 15 min vibrotactile stimulation. Black circle is right side and white circle is left side. 
Effect of Salivation by Facial Somatosensory Stimuli of Facial Massage and Vibrotactile... DOI: http://dx.doi.org/10.5772/intechopen.88495

\subsection{RR interval of ECG and total salivation}

First, we examined the difference between stranger and self-massages of the RR intervals of ECG. However, there were no differences between the RR intervals of ECG of stranger and self-massages. Especially, the RR intervals in 3 min stranger massage had a tendency to decrease (increase of heart rate), and there was a $p<0.01$ significance (T-test), as shown in Figure 4A. Namely, for 3 min of stranger massage, RR intervals increased from about 700 to $1000 \mathrm{~ms}$, and it evoked feeling of sleepy in everybody, as shown in Figure 4A. However, a $89 \mathrm{~Hz}$ vibrotactile stimulation was spent for $15 \mathrm{~min}$ to increase from about 700 to $1000 \mathrm{~ms}$ of RR interval, as shown in Figure 4A. Especially, immediately after the insert of cotton rolls, RR intervals showed about $700 \mathrm{~ms}$ and then after a few min increased up to about 900-1000 ms, as shown in Figure 4A. In particular, hand massages are effective to increase temperature and RR intervals. However, no change of total salivation did not show the effect of the autonomic system in 3 min hand massage.

However, hand massages, especially stranger one, will evoke sleepiness by the increased temperature, and metabolism will decrease getting sleepy with the non-increased salivation. Especially, hand massage is needed to countermeasure the effectiveness of fight falling asleep.

On the other hand, total salivation showed about $1.2 \mathrm{ml}$ in resting phase during $3 \mathrm{~min}, 0.5 \mathrm{ml}$ in stranger massage during $3 \mathrm{~min}, 1.0 \mathrm{ml}$ in self massage during $3 \mathrm{~min}$, and $3.5 \mathrm{ml}$ in $89 \mathrm{~Hz}$ vibrotactile stimulation during $15 \mathrm{~min}$, as shown in Figure 4B. Although 3 min hand massages did not show the effect of autonomic activity, 15 min vibrotactile stimulation did it.

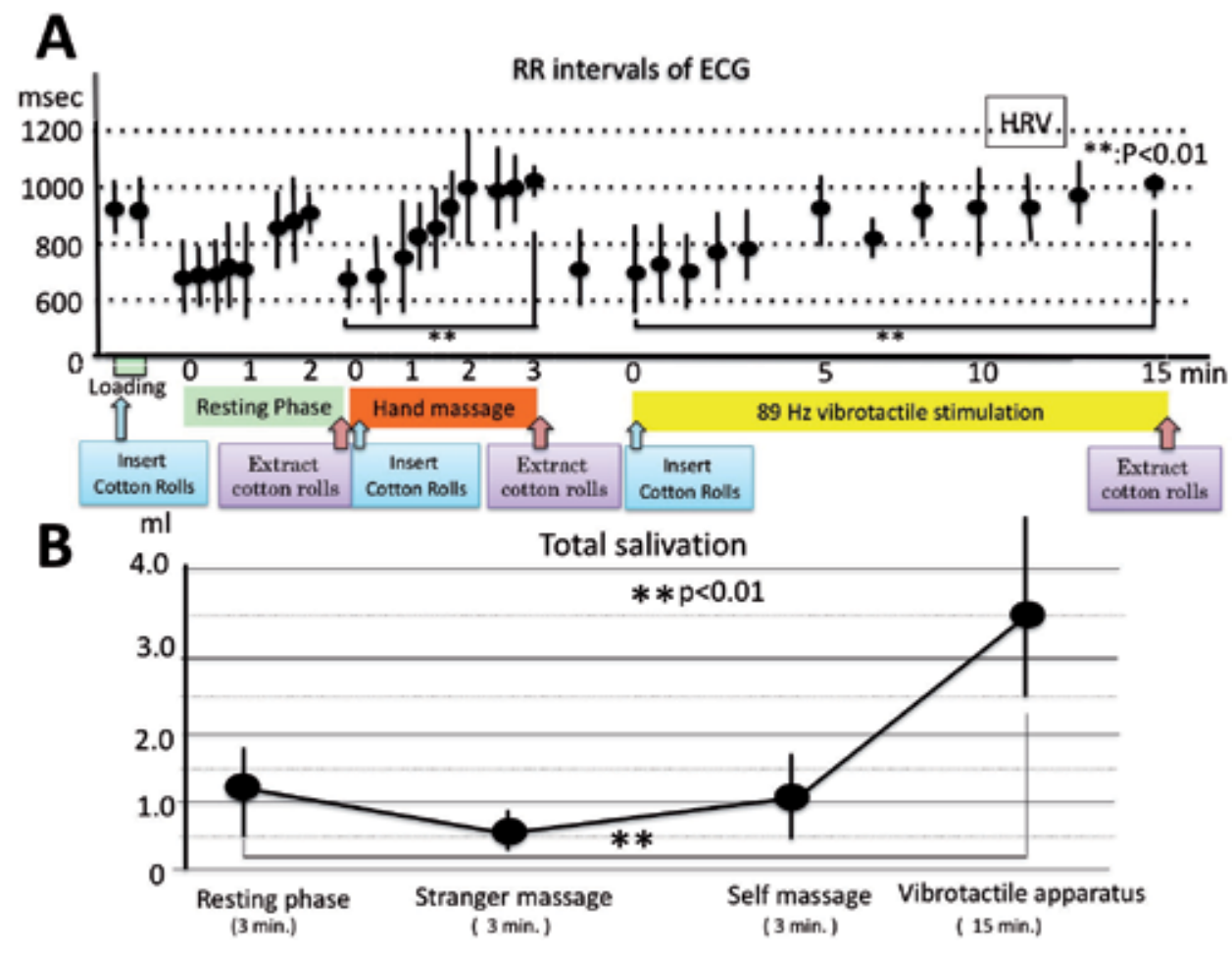

Figure 4 .

RR intervals of ECG $(A)$ and total salivation $(B)$ before and after 3 min hand (stranger and self-massage) massage. 


\subsection{Measurement of OxyHb of fNIRS on the frontal cortex}

The increase toward plus direction of $\mathrm{OxyHb}$ is associated with neuronal activities, but when we become sleepy, it is decreased toward below zero $[12,13]$. On the other hand, the zero level of OxyHb of fNIRS showed parasympathetic activity from our data [5-7]. According to the amount of OxyHb of fNIRS, $\mathrm{OxyHb}$ in the stranger massage showed the decrease below zero, as shown in Figure 5. The results may be sleepy with decreased $\mathrm{OxyHb}$ during the stranger massage. According to analysis of $\mathrm{OxyHb}$ activity of fNIRS, during the resting phase and hand stimulation, subjects showed the nonzero number, and during the second half stimulation of $89 \mathrm{~Hz}$ vibrotactile apparatus, they showed the zero level. In particular, the below zero level of OxyHb activity coincided with the inducing of sleepy in the stranger massage. We think that the nonzero number is the decreased metabolism with getting sleepy, and the zero level is the parasympathetic activity (2-15 $\mathrm{min}$ in $89 \mathrm{~Hz}$ vibrotactile stimulation), as shown in Figure 5 and the previous papers [5-8]; namely, the stranger massage will produce an early excitation ( $0-2 \mathrm{~min})$ and a late relaxation $(3 \mathrm{~min})$, as shown in Figure 5 .

\subsection{Measurement of amylase activity}

In the previous studies $[10,11,14]$, measured values with salivary amylase monitor (Nipro Co.) showed the increased value depending on the sympathetic activity; namely, the value of amylase activity measured by salivary amylase monitor is shown as an increase of value following increased sympathetic activity. The results showed that the minimum value was before hand massage (about $15 \mathrm{KIU} / \mathrm{L}$ ) and then the maximum value was after stranger massage (about $40 \mathrm{KIU} / \mathrm{L}$ ) and vibrotactile apparatus (about $42 \mathrm{KIU} / \mathrm{L}$ ), as shown in Figure 6. These findings show that subjects (patients) may be excited (decreased RR intervals, as shown in Figure 4A) by stranger's hand touching (Figure 4B). Furthermore, it may be related to an amount of amylase.

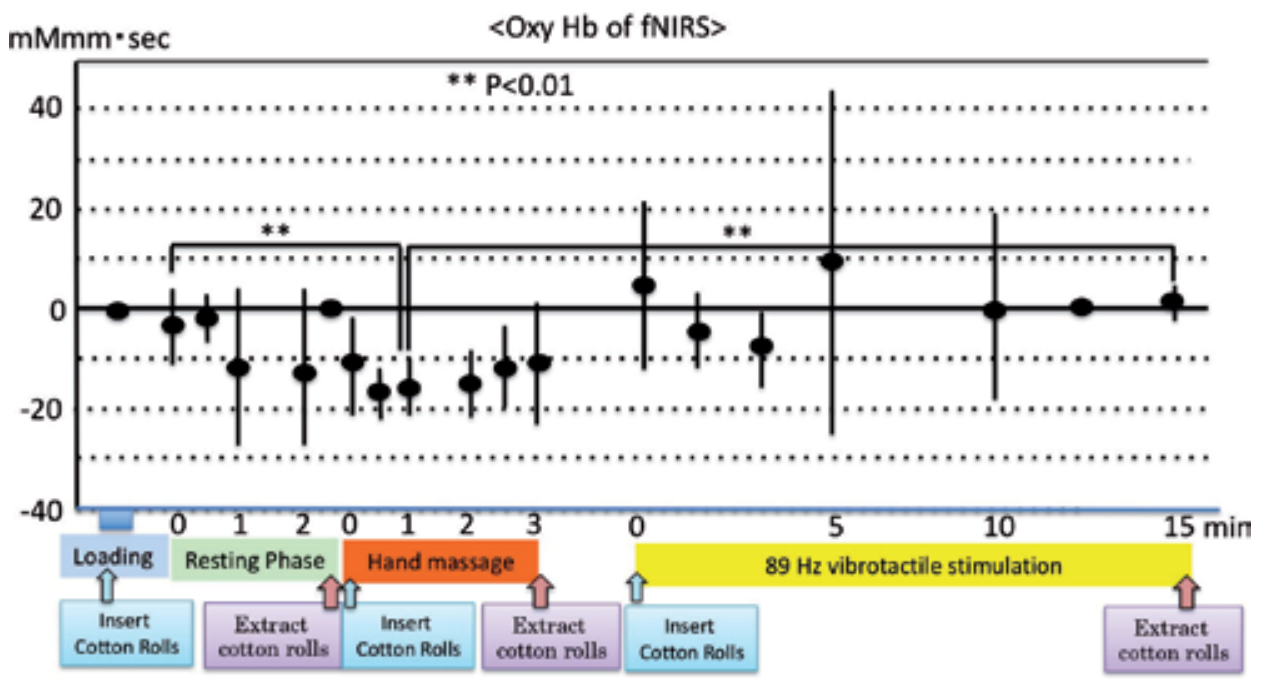

Figure 5.

Changes in $\mathrm{OxyH}$ of $\mathrm{fNIRS}$ of resting and stranger massage for 3 min, and vibrotactile stimulation of $89 \mathrm{~Hz}$ frequency and $9.8 \mu \mathrm{m}$ amplitude for 15 min. $p<0.01, p<0.05$, t-test. 


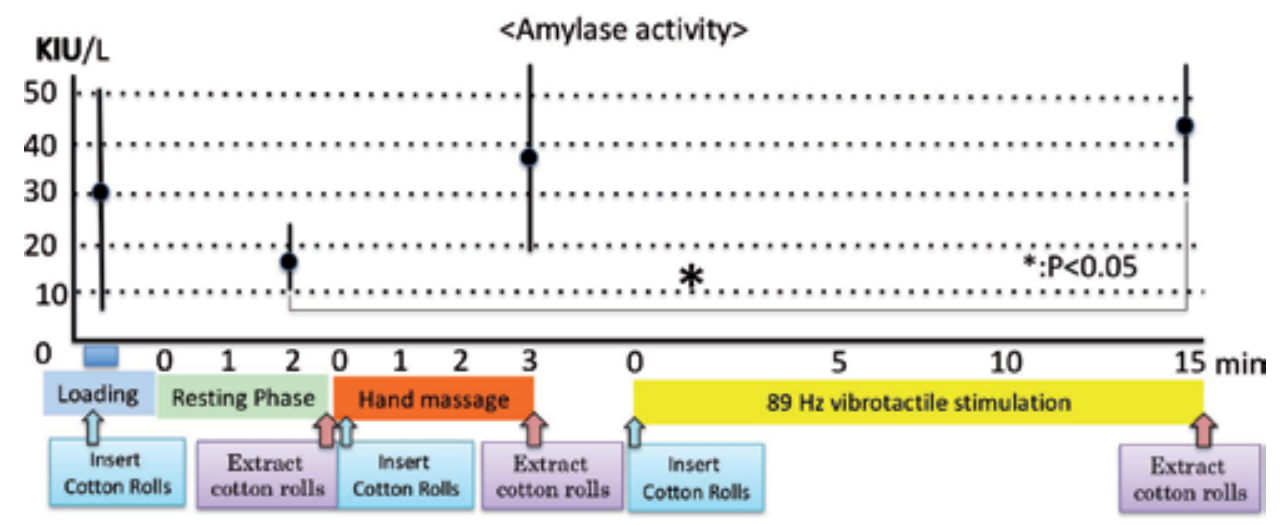

Figure 6.

Changes in amylase activities of loading, resting phase, and hand stranger massage for 3 min, and vibrotactile stimulation of $89 \mathrm{~Hz}$ frequency and $9.8 \mu \mathrm{m}$ amplitude for $15 \mathrm{~min}$.

\section{Discussion}

\subsection{Facial skin temperature}

Firstly, although we tried to perform 5 min massage, there were three bad deficits: in 5 min massage, operators complained about being very tired and they cannot handle patients with handicaps and stroke. Furthermore, we performed by the measurement of salivation with the cotton roll method. When we measured the amount salivation of a cotton roll, subjects of 5 min stimulation with heavy salivation often leaked from a cotton roll [8]. So, we focused to 3 min stranger hand massage, and we measured facial skin temperatures after 3 min resting phase and after 3 min stranger hand stimulation phase. In 3 min stranger hand stimulation, facial skin temperature increased about $2.0^{\circ} \mathrm{C}$, as shown in Figure 3. In particular, in comparison with before and after facial skin temperatures between resting and hand massage, although the resting phase increased as little as possible, the hand massage showed a big increase in temperature. On the other hand, a $89 \mathrm{~Hz}$ vibrotactile stimulation was spent for about $15 \mathrm{~min}$ to increase temperature (about $1.0^{\circ} \mathrm{C}$ ), as shown in Figure 3. The finding showed that the stranger hand massage was especially effective in facial skin temperatures. As shown in Figure 3, a $89 \mathrm{~Hz}$ vibrotactile stimulation was spent for $15 \mathrm{~min}$ for an increase of $1^{\circ} \mathrm{C}$ [5-8]. However, hand massages are adequate with $2-3 \mathrm{~min}$ for an increase of $1^{\circ} \mathrm{C}$, as shown in Figure 3 . Although a $89 \mathrm{~Hz}$ vibrotactile stimulation needs long time of 4-7 years as effective recovery, hand massage for 3 min may have more effect with a repetition of day after day, as shown in the previous paper [1]. A $89 \mathrm{~Hz}$ vibrotactile stimulation was spent for $15 \mathrm{~min}$ time for an increase of $1^{\circ} \mathrm{C}$, and this apparatus got effective by using at morning and night. However, as hand massage was necessary for 1-2 min, we may get effect by doing at morning and night; namely, an effective increase in facial skin temperature may be elicited by a good metabolism for recovery [15].

\subsection{Effect of autonomic activity}

The effect of autonomic activity can directly be studied by changes in RR intervals of ECG and total salivation. The RR interval and salivation are controlled by an autonomic activation, and the increased RR intervals (decrease of heart rate) and a great deal of serous salivation are parasympathetic activity and the 
decreased ones (increase of heart rate) and a little mucus salivation are sympathetic activity $[16,17]$. In the experimental room, the RR interval showed about $700 \mathrm{~ms}$ in the resting phase between 0 and 1 , and after the resting phase of the latter half, it arrived in about $900 \mathrm{~ms}$, as shown in Figure 4A. This insert of cotton rolls was activated by sympathetic nerve, and the RR intervals were decreased. Furthermore, in the resting phase of the latter half, it became naturalized, and the RR intervals increased, as shown in Figure 4A; namely, it showed the adaptation to the environment in latter resting phase. On the other hand, total salivation was decreased before and after hand massages, as shown in Figure 4B. This decrease may be activated by sympathetic nerve. Three minutes hand massages may be a small amount for the effective autonomic activity and an adequate massage times may produce an effective effort of the autonomic nerve. In the $3 \mathrm{~min}$ hand stimulation, they showed the increased rate in average values, and they showed significant differences (T-test, $p<0.01$ ) in RR intervals. However, in total salivation, the hand massage may not show the effect of the autonomic nerves as 3 min stimulation. Although this finding may be an effective treatment, we thought inadequate recovery of a function for salivation. We reported the increased salivation depending on the $89 \mathrm{~Hz}$ vibrotactile stimulation on the facial skin [1,5-7]. Namely, autonomic changes of stranger massage may be produced by an adequate stimulation time. However, the stranger hand massage did not show the total salivation, and always caused sleepiness after the experiments. We think increased temperature will show a good increased metabolism in stranger massage, but all of subjects are set to sue for sleepiness in the stranger massage. Namely, it is important to get not to sleep for prevention of a decline in metabolism.

\subsection{Analysis of OxyHb of fNIRS on the frontal cortex}

We have studied an analysis of OxyHb of fNIRS (a functional near-infrared spectroscopy) on the frontal cortex during vibrotactile stimuli, as shown in the previous papers $[1,5-8]$. As a result, we reported that the zero level of the OxyHb of fNIRS showed the parasympathetic activity (Figure 5), and total salivation in the vibrotactile stimulation of the $89 \mathrm{~Hz}$ frequency and $9.8 \mu \mathrm{m}$ amplitude were the most effective.

Facial skin temperature increased from 32 to $34^{\circ} \mathrm{C}$ spend $3 \mathrm{~min}$ in the hand massage, and did $15 \mathrm{~min}$ in $89 \mathrm{~Hz}$ vibrotactile stimulation. Although $3 \mathrm{~min}$ stranger massage did not show the effective effect of total salivation, RR intervals increased from 670 to $1000 \mathrm{~ms}$. However, over $3 \mathrm{~min}$ massage evoked the fatigue feeling. So, we tried $15 \mathrm{~min} 89 \mathrm{~Hz}$ vibrotactile stimulation. To increase RR intervals, we spent $3 \mathrm{~min}$ in the hand massage, but $15 \mathrm{~min}$ was spent in the $89 \mathrm{~Hz}$ vibrotactile stimulation; namely, an increase of facial skin temperature and RR interval will be effective in the 3 min hand massage.

We measured facial skin temperature and fNIRS on the frontal cortex during 3 min hand stimulation and during 3 and $15 \mathrm{~min} 89 \mathrm{~Hz}$ vibratory stimulation. We reported that the vibrotactile stimuli on the facial skin showed the near zero level of values of OxyHb, DeoxyHb, and TotalHb in fNIRS $[1,5-8]$. The OxyHb and DeoxyHb of brain circulation in the frontal cortex were reported to parallel the neuronal activity [2]. The finding is showed by the increased parasympathetic activity $[1,5-8]$. On the other hand, the decreased OxyHb (under zero level) showed the decreased neuronal activity with the decreased consumption of oxygen and metabolism, and the drowsy and sleepy conditions were shown by the decreased $\mathrm{OxyHb}$ during hand massages [13]. According to OxyHb activities of fNIRS on the frontal cortex, effects between stranger massage and resting condition are almost the same under zero level. Hand stimulation for $3 \mathrm{~min}$ may not show the increase of 
total salivation, except for the increase of facial skin temperatures, RR intervals of ECG and saliva amylase. However, RR interval and salivation were effective effort on $15 \mathrm{~min} 89 \mathrm{~Hz}$ vibrotactile stimulation, as shown in Figure 4B and the previous paper [5]. As over 3 min massage produced the fatigue feeling, we thought that repetitious stimuli may change the autonomic system and during hand massage, we tried hard not to get sleepy by talking. However, in the subject, this active feeling was decreased immediately and the subject felt sleepy.

\subsection{Changes in an activity of saliva amylase}

A stress is activated by the activation of sympathetic nerve, and an activity of saliva amylase is increased by a self-preservation response of a body; namely, the activity of amylase shows the activity of sympathetic nerve [14]. However, we may relate to amount of amylase because of their mass production after 15 min vibrotactile stimulation, as shown in Figure 6. The results showed the increased activation of saliva amylase related to amount of amylase, too. So, the increased saliva amylase was exhibited by about increase 2.5 times in $3 \mathrm{~min}$ hand stimulation and $89 \mathrm{~Hz}$ vibrotactile stimulation showed three times, as shown in Figure 6. This finding shows that subjects may be excited by stranger's hand touching and then decrease of RR intervals (Figure 4B). On the other hand, it may be related to increase of amylase activity and amount of saliva in the $89 \mathrm{~Hz}$ vibrotactile stimulation, too.

\section{Conclusion}

Generally, a massage is an immediate recovery of reducing stress with warm feeling by a light touch. An explanation in Japanese textbook of "salivation glands massage" was caused by extrusion of accumulated salivary from the acinus glands. However, this method of salivation massage was not encouraged by shakeout of poor glands, we thought. Traditional idea of massage shows immediate recovery of reduced stress with warm feeling. Namely, traditional massage on the facial skin shows immediate reaction, and deactivated salivary glands will be a recovery with an improvement of circulating blood by the increased temperature [18]. The increasing temperature by hand stimuli brings forward metabolism in the facial skin and saliva glands, and deactivated saliva glands as a recovery will be a good tendency. We focused on $3 \mathrm{~min}$ hand stranger massage, because $5 \mathrm{~min}$ massage was provoked by feeling of fatigue, and self-massage could not treat in handicap and stroke patients. However, we were performed by a hand stranger massage for $3 \mathrm{~min}$, because of operator's fatigue over $3 \mathrm{~min}$ massage. In the effect of automatic nerve system, the hand stranger massage for 3 min increased RR intervals of ECG, did not increase salivation, and increased the amylase activity. However, the hand stranger massage was the best effectiveness of the increased facial skin temperature. On the other hand, hand massages will evoke sleepiness by the increased temperature, and metabolism will decrease getting sleepy with the non-increased salivation. Furthermore, $\mathrm{OxyHb}$ of fNIRS on the frontal cortex showed values below zero during hand stimulation. Values below zero in OxyHb of fNIRS show the sleepiness. This reason may suggest that hand massage is effective not only due to increased temperature and metabolism but also non-increased salivation and heart rates. In particular, during hand massage, we must try not to get sleepy by talking. However, the vibrotactile stimulus apparatus for 15 min showed changes in the RR interval of ECG and salivation. On the other hand, repetitious stimuli may change the autonomic system, but the most suitable time of repetitious intervals is necessary for further experiments. As shown in Figure 4A-a, -b, a $89 \mathrm{~Hz}$ vibrotactile stimulation is spent for $15 \mathrm{~min}$ for an increase 


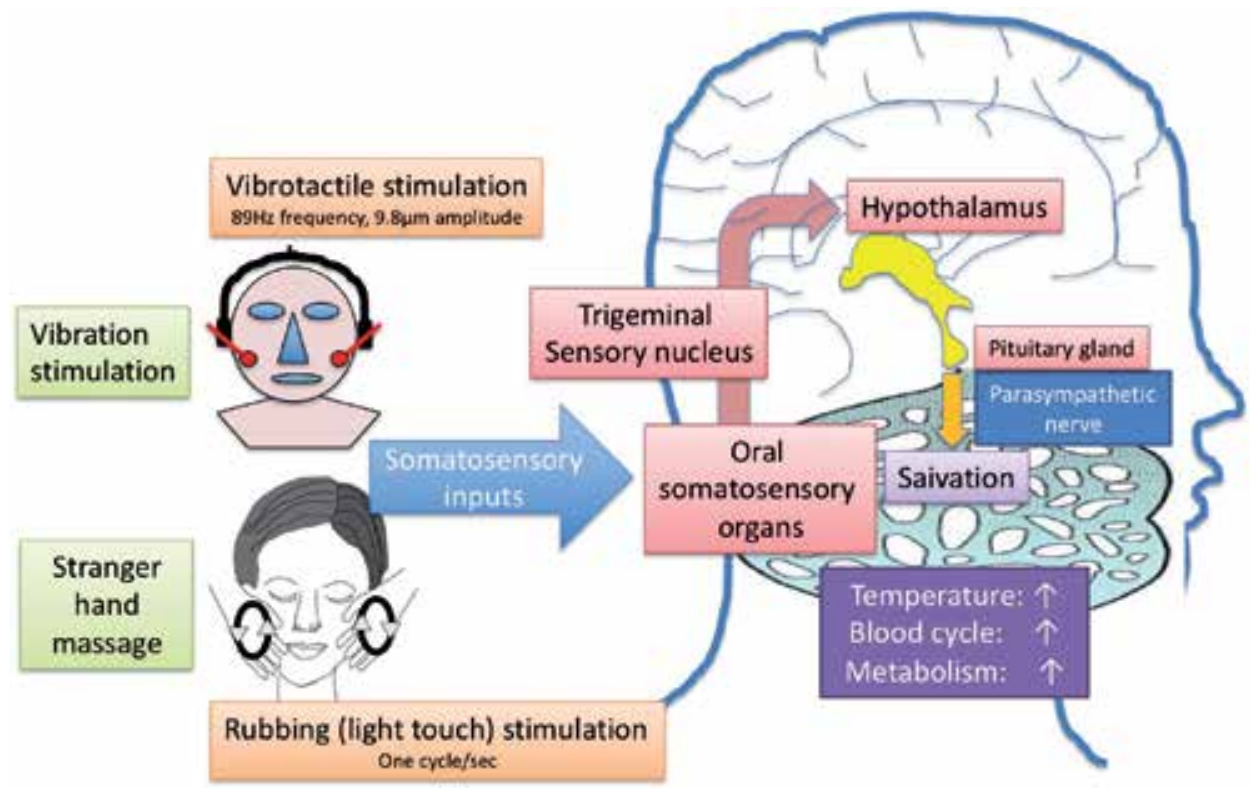

Figure 7.

Our idea about effects in salivation of facial somatosensory inputs. In summary, we showed our idea of salivation by facial somatosensory stimuli, hand stranger massage, and vibrotactile stimulation. Facial somatosensory sense was excited by oral somatosensory inputs with the $89 \mathrm{~Hz}$ vibrotactile stimulation or hand massage. Vibrotactile inputs arrived at the hypothalamus via the trigeminal somatosensory nucleus, and then parasympathetic nerves were activated and produced salivation. So, vibrotactile stimulation will be slowly recovered with the increase of facial skin temperature. Although vibrotactile stimulation spent many time for recovery of glands, hand massage might do a short time for recovery. In particular, the hand stranger massage rapidly increased the produced facial skin temperature and reducing stress. Furthermore, it will recover circulation and metabolism. This massage may be early recovered by a repetitious performing in comparison with a recovery period of the vibrotactile apparatus.

of $1^{\circ} \mathrm{C}$ [5-8]. However, hand massages are adequate with 2-3 min for an increase of $1^{\circ} \mathrm{C}$, as shown in Figure $2 \mathrm{~A}$ and $\mathbf{B}$. Although the $89 \mathrm{~Hz}$ vibrotactile stimulation needs long time of 4-7 years for effective recovery, hand massage for 3 min may have more effect with a repetition of day after day. Namely, an effective increase of facial skin temperature may be elicited by a good metabolism for recovery.

In summary, we showed our idea of salivation by facial somatosensory stimuli, hand massage, and vibrotactile stimulation as shown in Figure 7. Facial somatosensory sense was excited by oral somatosensory inputs with the $89 \mathrm{~Hz}$ vibrotactile stimulation or hand massage. Vibrotactile inputs arrived at the hypothalamus via the trigeminal somatosensory nucleus, and then parasympathetic nerves were activated and produced salivation. So, vibrotactile stimulation will be slowly recovered with the increase of facial skin temperature. Although vibrotactile stimulation spends many time for recovery of glands, hand massage might do a short time for recovery. In particular, the hand massage rapidly increased the produced facial skin temperature and reduced the stress. Furthermore, it will recover circulation and metabolism. This massage may be early recovered by a repetitious performing in comparison with a recovery period of the vibrotactile apparatus.

\section{Acknowledgements}

This work was supported by Sato (2018) and Sogoshigaku (2018) research grant of Nihon University School of Dentistry, by Grants-in-Aid for Scientific Research (21592539) and by a grant from the Ministry of Education, Culture, Sports, Science, and Technology to promote multidisciplinary research projects. 
Effect of Salivation by Facial Somatosensory Stimuli of Facial Massage and Vibrotactile...

DOI: http://dx.doi.org/10.5772/intechopen.88495

\section{Conflict of interest}

None of the authors report any conflict of interest.

\section{Ethical approval}

The Nihon University School of Dentistry provides ethical approval to conduct this pilot clinical study (approval number: 2009-5). All procedures performed in studies involving human participants were in accordance with the ethical standards of the institutional and/or national research committee and with the 1964 Helsinki declaration and its later amendments or comparable ethical standards. This chapter does not contain any studies with animals performed by any of the authors.

\section{Informed consent}

Informed consent was obtained from all individual participants included in the study.

\section{A. Appendices and nomenclatures}

Obey dysphasia rehabilitation society.

\section{Author details}

Tsunoda Yumi ${ }^{1}$, Akatuka Sumiko ${ }^{1}$, Fukui Sayaka ${ }^{1}$, Nakayama Enri ${ }^{2}$, Abe Kimiko ${ }^{2}$, Sato Mituyasu², Kimura Masanori², Kato Syunnichiryou ${ }^{2}$, Sakai Maho ${ }^{2}$, Yamaoka Masaru ${ }^{3}$, Watanabe $\mathrm{Mao}^{2}$, Ueda Koichirou ${ }^{2}$ and Hiraba Hisao ${ }^{2 *}$

1 Nihon University Dental Hospital at Surugadai, Chiyoda-Ku, Tokyo, Japan

2 Department of Dysphasia Rehabilitation, Chiyoda-Ku, Tokyo, Japan

3 Department of Physics, Chiyoda-Ku, Tokyo, Japan

*Address all correspondence to: hiraba60@gmail.com

\section{IntechOpen}

(C) 2019 The Author(s). Licensee IntechOpen. This chapter is distributed under the terms of the Creative Commons Attribution License (http://creativecommons.org/licenses/ by/3.0), which permits unrestricted use, distribution, and reproduction in any medium, provided the original work is properly cited. (cc) BY 


\section{References}

[1] Ueda K, Gora K, Yamaoka M, Abe K, Nakayama E, Sato M, et al. Chapter 7: Salivary effects of facial vibrotactile stimulation patients with Sjogren's syndrom and poor salivation. In: Guvenc IA, editor. Salivary GlandsNew Approaches in Diagnostics and Treatment. Rijeka, Croatia: IntechOpen; 2017. pp. 105-116. Available from: http:// www.intechopen.com

[2] Ito K. Koukuu kannsou syou (dry mouth). In: Saito E, Mukai Y, editors. Sessyoku Ennge Rehabilitation. Tokyo: Ishiyaku Co.; 2009. pp. 96-98. (in Japanese)

[3] Kakigi Y. Koukuukannsousyou enotaiou. In: Morito M, editor. Koureisha Shika Gaku. Kyoto: Nagasue Syotenn; 2014. pp. 75-78. (in Japanese)

[4] Amelia DA. Chapter 2: Loving touch. In: Baby Massage: Parent-Child Bonding through Touch. New York: Newmarket Press; 1989. pp. 25-40

[5] Hiraba H, Inoue M, Gora K, Sato T, Nishimura S, Yamaoka M, et al. Facial vibrotactile stimulation activates the parasympathetic nervous system: Study of salivary secretion, heart rate, pupillary reflex, and functional nearinfrared spectroscopy activity. BioMed Research International. 2014;2014:1-9

[6] Hiraba $H$, Inoue M, Sato T, Nishimura S, Yamaoka M, Shimano T, et al. Chapter 14: Optimal vibrotactile stimulation activates the parasympathetic nervous system. In: Francisco B-C, editor. Advances in Vibration Engineering and Structural Dynamics. Rijeka, Croatia: IntechOpen; 2012. pp. 335-369. Available from: http://www.intechopen.com

[7] Hiraba H, Sato T, Nishimura S, Yamaoka M, Inoue M, Sato M, et al. Chapter 16: Changes in brain blood flow on frontal cortex depending on facial vibrotactile stimuli. In: Beltran-Carbajal F, editor. Vibration Analysis and Control-New Trends and Developments. Rijeka, Croatia: IntechOpen; 2011. pp. 337-352. Available from: http://www.intechweb.org

[8] Hiraba H, Yamaoka M, Fukano Y, Fujiwara T, Ueda K. Increased secretion of salivary glands produced by facial vibrotactile stimulation. Somatosensory \& Motor Research. 2008;25:222-229

[9] George E, Billman A. Heart rate variability-A historical perspective. Frontiers in Physiology. 2014;2:1-13

[10] Gopal KS, Seena U, Shradha MP. Salivary alpha amylase activity in human beings of different age groups subjected to psychological stress. Indian Journal of Clinical Biochemistry. 2014;29:485-490

[11] Kodama T, Abe T, Kanehira T, Morita M, Funahashi M. Analysis of fluctuations of stress markers in saliva. Hokkaido Journal of Dental Science. 2010;31:52-61. (in Japanese)

[12] Mackert B-M, Leistner S, Wahi MM. Dynamics of cortical neurovascular coupling analyzed by simultaneous DC-magnetoencephalography and timeresolved near-infrared spectroscopy. NeuroImage. 2008;39:979-986

[13] Suda M, Sato T, Kameyama M. Decreased cortical reactivity underlies subjective daytime light sleepiness in healthy subjects: A multichannel near-infrared spectroscopy study. Neuroscience Research. 2008;60:319-326

[14] Masaki Y. Development of salivary control sensor including big data analysis. Autonomic Nerve. 2014;51:140-146. (in Japanese) 
Effect of Salivation by Facial Somatosensory Stimuli of Facial Massage and Vibrotactile...

DOI: http://dx.doi.org/10.5772/intechopen.88495

[15] Muller MD, Ryan EJ,

Bellar DM, Kim CH, Blankfield RP, Muller SM, et al. The influence of interval versus continuous exercise on thermoregulation, torso hemodynamics, and finger dexterity in the cold. European Journal of Applied Physiology. 2010;109:857-867

[16] Ivarsen S, Ivarsen L, Saper CB. Chapter 49: The autonomic nervous system and the hypothalamus. In: Kandel ER, Schwartz JH, Jessell TM, editors. Principles of Neural Science. 4th ed. New York: McGraw-Hill; 2000. pp. 960-981

[17] Ivarsen S, Kupfermann F, Kandel ER. Chapter 50: Emotional states and feelings. In: Kandel ER, Schwartz JH, Jessell TM, editors. Principles of Neural Science. 4th ed. New York: McGraw-Hill; 2000. pp. 982-997

[18] Gardner EP, Martin JH. Chapter 21: Coding of sensory information. In: Kandel ER, Schwartz JH, Jessell TM, editors. Principles of Neural Science. 4th ed. New York: McGraw-Hill; 2000. pp. $411-429$ 

Section 7

\section{Dysphagia Due to Cervical and Facial Tumors}





\title{
Swallowing Disorders in Cervical Facial Tumors
}

\author{
Daniela Vrinceanu and Mihai Dumitru
}

\begin{abstract}
We review current state of the art protocols on swallowing disorders associated to cervical facial tumors. The clinician needs to translate physiology notions to bedside diagnosis. Facing such a case the ENT surgeon must follow several key steps: thorough history taking, barium transit, endoscopy evaluation of swallowing, high resolution diagnosis imaging. Afterwards surgical treatment plan should take into consideration the need to careful dissection of vascular and nervous structures. Dysphagia may present from initial diagnosis or after surgical resection of the tumor or during radiation and chemotherapy. We discuss the use of various staging scales or questionnaires for assessing quality of life. We illustrate the importance of swallowing disorders management with various cases of tumors at the level of skull base, pharynx, salivary glands, larynx, esophagus, etc. There are various solutions for dysphagia ranging from nasogastric feeding tube placement to percutaneous endoscopic gastrostomy to specially designed exercises. Sometimes the surgeon neglects these disorders and focuses on airway management. However, the rule should be to encourage swallowing as soon as possible after surgery. A good nutritional status is necessary for a positive prognosis in swallowing disorders. Team effort in tertiary oncology units is the key in supporting such complex cases.
\end{abstract}

Keywords: tumors, cervical, facial, swallowing, disorders

\section{Introduction}

Pharyngeal dysphagia is defined as a disorder affecting the passage of food from the oral cavity to the stomach. When swallowing associates pain it is described as odynophagia. Tumors at the level of head and neck frequently associate dysphagia and odynophagia [1].

Swallowing disorders represent the first sign of tumor pathology in upper aerial and digestive tract. In $14-18 \%$ of the cases the isolated symptom of dysphagia requires a complete screening for primary occult tumors [2].

Surgical removal of cervical and facial tumors hinders swallowing process by removing parts from the pharyngeal muscles or nerves. Even for removal of benign tumors there may be inflicted lesions of cranial nerves responsible with swallowing and lowering the quality of life of the patient mostly because reconstruction achieves structural continuity but not function. Moreover, radiotherapy and chemotherapy lead to oral mucositis hindering correct swallowing [3].

After completing therapy, we can define a residual dysphagia. Unfortunately swallowing disorders become permanent in $40 \%$ of cases, diminishes in $39 \%$ of the 
cases and worsens in 20\%. In all these cases dysphagia needs further evaluation and require prevention of aspiration and improvement of quality of life [4].

In cervical facial tumors, dysphagia is connected to factors such as: direct extension of the tumor, aggressiveness of the surgical resection, radiotherapy and chemotherapy. Swallowing disorders may represent an element of diagnosis, an indicator of the postsurgical recovery and an important factor the quality of life [5].

\section{Clinical physiology of swallowing}

The swallowing process presents three stages: oral, pharyngeal and esophageal. All these stages are marked by activation of specific anatomical and functional connections enabling early diagnostic topography of dysphagia [6].

Oral stage is the only voluntary moment in swallowing. Aliments are submitted to mechanical transformation during mastication and mixture with saliva thus forming the alimentary bolus. Masseter muscles are central in mastication and are innervated by the motor branch of the trigeminal cranial nerve. Consistency of the bolus is also important during swallowing process enabling the correct backward movement towards the pharynx due to the pressure of the tongue on the soft palate. From this point swallowing is becomes uncontrolled and automatic [7].

During pharyngeal stage the bolus stimulates receptors at the level of the anterior region of the pharyngeal tonsils. Impulses from this level reach the brainstem initiating a series of reflex muscular contractions with simultaneous purposes: propulsion of the bolus through digestive tract and protection of superior airways. Therefore, the soft palate is stiffened closing the choana; palatal-pharyngeal arches are medial delineating a sagittal slit through which the bolus passes into the pharynx; epiglottis moves posterior over the laryngeal inlet; vocal cords adduct thus closing and the larynx is displaced anterior and upwards by external muscle groups [8].

All these contractions prevent the entrance of bolus in the larynx and trachea. Upwards movement of the larynx opens the esophageal inlet. Simultaneously the complex of the pharyngeal and superior esophageal sphincters relaxes enabling the passage from hypopharynx to superior esophagus. In the same sequence the entire posterior pharyngeal wall records the contraction of middle and inferior pharyngeal constrictor muscles towards the esophagus. All these processes last only 1-2 seconds [9].

Esophageal stage presents primary peristalsis waves and secondary peristalsis waves transporting the bolus till the stomach. Primary peristalsis continues the pharyngeal contraction and reaches the stomach in 8-10 seconds. Secondary peristalsis waves are generated by esophageal distension. These are initiated by the esophageal plexus and reflexes with afferent vague fibers to the medulla oblongata [10].

All three stages unite the control from cranial nerves IX-XII and trigeminal and facial nerves. Therefore six cranial nerves and more than 25 muscles are responsible for swallowing completion [11].

\section{Neurology syndromes affecting cranial nerves}

Syndromes affecting the last four pairs of cranial nerves are frequently encountered in cervical facial tumors mostly when superior jugular lymph nodes enlarge due to metastasis and compress these nerves and posterior cervical sympathetic trunk. Other scenario is regarding the evolution of skull base tumors extending to posterior cranial fossa [12]. 
Vernet syndrome was described in 1916 and implies affliction of cranial nerves IX, X and XI. These jugular foramina syndrome presents with: paralysis of half of the soft palate, paralysis of laryngeal recurrent nerve, paralysis of sternocleidomastoid and trapezius muscles on the same side. As etiology possible is trauma or external tumor compression at the level of the jugular foramina [13].

When a patient with the aforementioned syndrome has further lesion of the XII cranial nerve, we describe the Collet-Sicard syndrome. Hypoglossal paralysis leads to deviation of the protruding tongue towards the affected part and atrophy of the tongue on the same side after 6 months [14].

Involvement of the sympathetic chain leads to Horner syndrome associating enophthalmia, ptosis, myosis, vascular disorders and redness and sweating on the same side of the face [15].

Tapia described as early as 1905 a syndrome affecting nerves X and XII with recurrent laryngeal nerve palsy and tongue palsy on the same side [16].

Causes of these syndromes could be trauma, inflammation or tumor compression. So, facing the case of an isolated neurological syndrome with dysphagia one must take into consideration the possibility of a primary occult or high secondary metastatic cervical tumor [17].

\section{Principles of positive diagnosis in swallowing disorders}

From the very beginning the specialist should focus on complete history taking and symptoms accurate recording, pharyngeal clinical exam and swallowing clinical exam [18].

History taking is a key element in diagnosis of swallowing disorders. One should record: pain during swallowing, provocation of cough during swallowing, avoiding food with higher consistency, weight loss, prior history of pneumonia and other neurological diseases. Should be very clear the beginning of dysphagia and associated symptoms such as dysphonia and dyspnea. Very important is the exposure to tobacco smoke and alcohol consumption. Moreover, it is important to record other neurological symptoms as presences of herpes simplex virus lesions, previous trauma or other associated diseases such as diabetes. Another aspect is the swallowing difficulty with solids or liquids, because solid dysphagia points to a possible tumor while dysfunction with liquid aliments is encountered mostly in neurology diseases [19].

Clinical ENT exam should be thorough with an extensive flexible fiber endoscopy for occult tumors at the level of the rhinopharynx, palatine tonsils, tongue base, and pharyngeal-laryngeal junction. Analyze the dental status, use of dental prosthesis, dryness of the mucosa, tongue, lips and soft palate symmetry. Tactile sensibility of lips, tongue and pharynx should be also assessed. Very important is correct palpation of the neck for lymph nodes enlargement and thyroid pathology. There are documented cases in which a large goiter can compress the esophageal inlet and thus lead to dysphagia. A consistent amount of time should be allotted to cranial nerves exam along with a complete neurology consult [20].

Discovering a swallowing disorder requires a controlled passage test with aliments of different textures [21].

\section{Objective analysis of swallowing disorders}

There is hardly a consensus regarding optimum evaluation of pharyngeal dysphagia with various strategies at level of diagnosis center or country. ICON 
2018 consensus querying five experts from four continents recommends functional endoscopic evaluation swallowing (FEES) and video fluoroscopic swallowing study (VFSS). FEES visualize the pharynx through a trans nasal endoscope and detect rapid movement of solid and liquid aliments with a high risk of aspiration. VFSS may be combined with conventional manometers and enables the correlation between motility and pressure patterns inside the lumen [22].

Also useful is barium transit which facilitates direct dynamic view of the swallowing process and aspiration of the contrast media in the larynx and trachea. Sometimes, the contrast media should be sterile and soluble for preventing aspiration lesions (Figure 1). Sometimes even a standard cervical column X-ray can reveal ossification of the anterior vertebral longitudinal ligament leading to Forrestier syndrome [23].

It is recommended the use of and objective evaluation scales such as the one designed by Carnell et al. According to this scale grade 1 is normal performance. Second grade is within functional limits with an abnormal oral stage but a normal diet. Third grade records mild impairment of the pharyngeal stage with a modified diet. Grade 4 requires further therapeutic precautions to minimize aspiration risk. Fifth grade notes the presence of aspiration. Grade 6 will require the use of enteral feeding support. Final grade presents with severe impairment and inadequate transit to esophagus [24].

This objective scale evaluates swallowing for introduction of parenteral nutrition and monitoring the patient evolution. The impact of swallowing dysfunction on quality of life is made through self-evaluating questionnaires. Such tools are: the swallowing questionnaire quality of life (SWAL-QOL) and SWAL-CARE and MD Anderson Dysphagia Inventory (MDADI). SWAL-QOL contains 44 questions reunited in several scores with the purpose of identifying patients with dysphagia. MDADI has only 20 questions with scales for emotional, functional and physical impact of dysphagia [25].

The best management decision takes into consideration both objective and subjective evaluations of swallowing dysfunction.

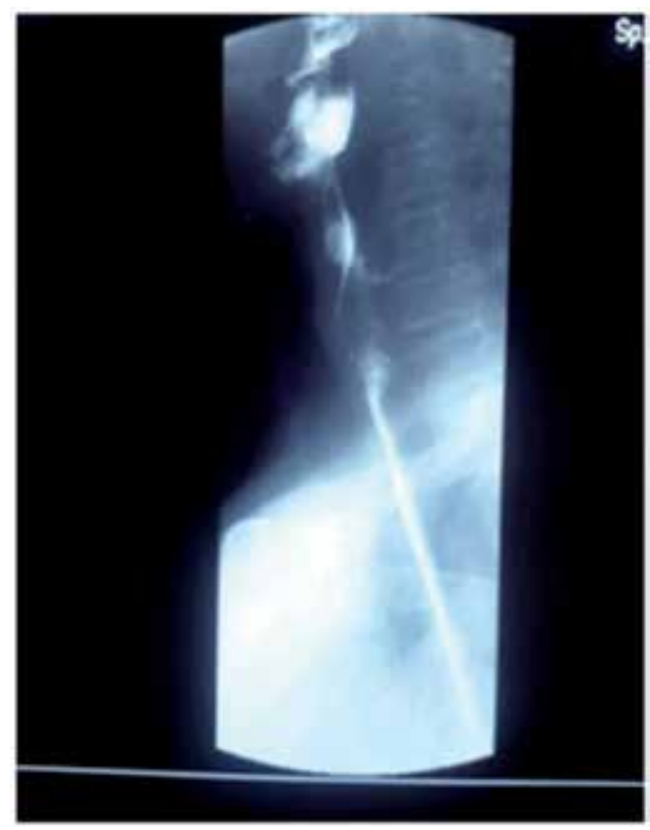

Figure 1.

Barium transit revealing obstruction and diminished passage of the contrast substance posterior to the larynx. 


\section{Imaging in swallowing disorders}

Given the history data and clinical exam the next management step should be based on cranial and cervical CT scan or MRI imaging. Imaging enables correct planning of biopsy which is the gold standard in diagnosing tumors at the level of head and neck. A distinct chapter is primitive metastatic lymph nodes which require a thorough imaging screening for detecting the original neoplasm [26].

Up to this point the work-up should follow careful history taking, complete clinical exam, multi-level flexible endoscopy, barium enema with sterile contrast media, head and neck CT scan or MRI, targeted biopsy with pathology exam.

Differential diagnosis of swallowing disorders focuses on tumors if upper aerodigestive tract or a head and neck tumor, neurology syndromes or degenerative neural disease, infectious diseases such as herpes zoster, stroke, cervical vertebra pathology and rare conditions such as dilation of esophageal inlet [27].

\section{Clinical aspects of dysphagia in cervical and facial tumors}

Malignant tumors of the tongue and mouth floor have an important effect on deglutition. Large resections of the tongue require mounting a nasopharyngeal feeding tube for up to 14 days after surgery. This tube is necessary for preventing resection margins dehiscence and preventing aspiration. During these 2 weeks period the patient performs small exercises in order to develop compensatory mechanisms. Coughing before removal of the tube should require prolongation of its use [28].

Regarding malignant tumors of tongue base, we encounter a low survival with a diminished quality of life (Figure 2). Mostly in these cases the only management pathway available is centered on radiotherapy. Gastrostomy is necessary to be maintained up to 6 months and its removal should be attempted only after clinical and imaging confirmation of remission and low probability of bolus aspiration [29].

Nonetheless during palliative oncologic treatment, the nutritional status is very important because symptoms such as oral mucositis may contribute to weight loss and treatment failure.

Parotid gland tumors both benign and malignant greatly influence swallowing through various mechanisms. For example, sectioning the facial nerve will lead to poor preparation of the alimentary bolus during mastication. In many cases this aspect is neglected by the specialist surgeon already troubled by the facial neurologic deficit [30].
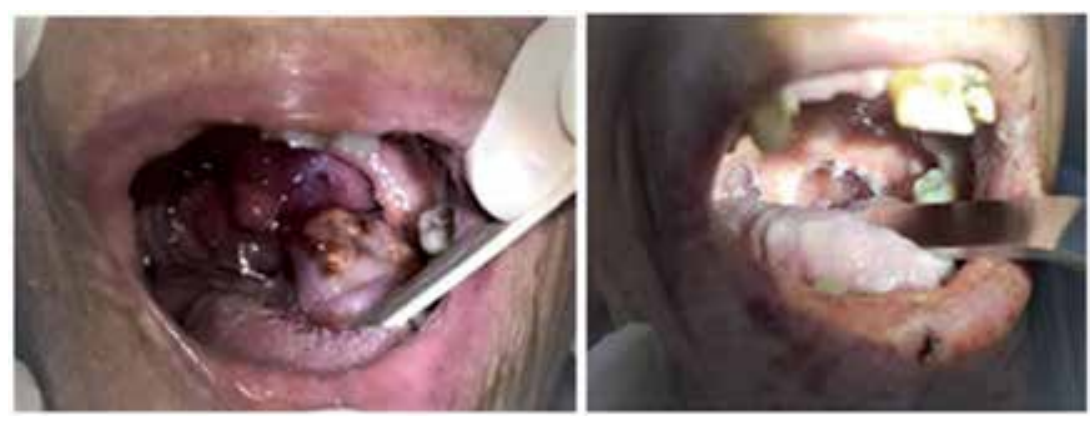

Figure 2.

Tumor in the left tongue base requiring resection for radical oncology surgery and thus leading to major swallowing dysfunctions. 
Pharyngeal tumors associate swallowing problems from the very beginning. Unilateral pain during swallowing should grow the suspicion of a palatine tonsil tumor. After surgery many of the swallowing deficits are corrected using specially design exercises and head positioning during food intake. Facing a tumor on the posterior of the pharynx the swallowing dysfunction has an early onset but neglected by patients coming from poor economic environments with deficit in healthcare coverage (Figure 3 ). The current treatment possibilities are reduced to radio and chemotherapy after placement of gastrostomy [31].

Tumors at the level of the retro cricoids region present rapid onset of swallowing difficulties with marked weight loss and usually without voice hoarseness (Figure 4). Their evolution has a bad prognosis due to the invasion of recurrent laryngeal nerves and development of a Gerhardt syndrome. Often the gastrostomy procedure is preceded by tracheotomy [32].

Esophageal tumors have a clinical debut with swallowing difficulties with a rapid progression. When attempting surgical removal of the tumor the surgeon must take into consideration the reconstructive process with stomach or colon prior to performing gastrostomy. In these cases, the most difficult aspect is the risk of fistula and the cooperation of the patient during exercises in order to prevent aspiration of alimentary bolus [33].

Laryngeal tumors and their extension to the pharynx require total laryngectomy procedures with partial pharyngectomy and preservation during surgery of the middle and inferior constrictor pharyngeal muscles and negotiating between radical oncology and economy of the pharynx mucosa (Figure 5) [34].

Reconstruction of the new pharyngeal junction over the placement of a nasogastric tube held in place up to 14 days. Development of a salivary fistula implies the prolonged use of the nasogastric tube and forbidding any swallowing even for saliva in order to expedite healing. Correct food regimen through the probe is important in assuring the nutritional support to scar healing. After removal of the nasogastric tube after total laryngectomy it will be given only half solid aliments able to pass through the new pharyngeal inlet without the use of the muscular fibers removed during surgery. Patients undergoing vocal training also surpass swallowing disorders quicker in the next 3 months [35].

Lymph node metastasis can generate swallowing disorders due to compression over cranial nerves IX-XII (Figure 6). Ideally during neck dissections proceed carefully in order to preserve hypoglossal nerve [36].

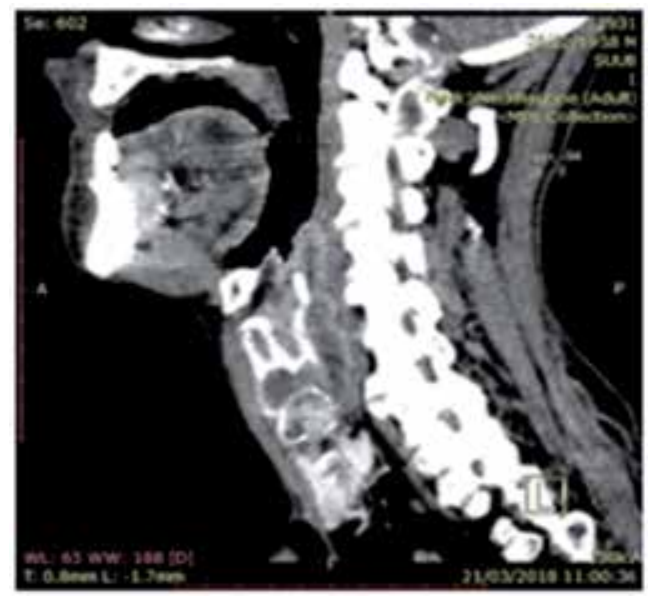

Figure 3.

Carcinoma of the posterior wall of the pharynx with complete dysphagia. 


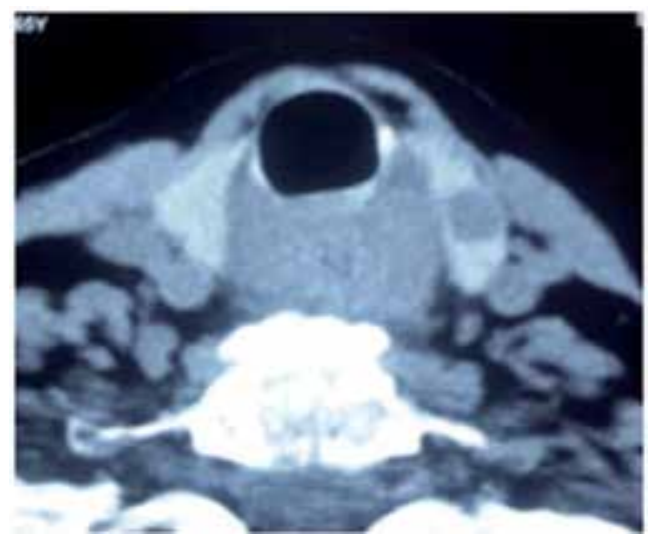

Figure 4 .

Tumor behind cricoids cartilage with complete obstruction.
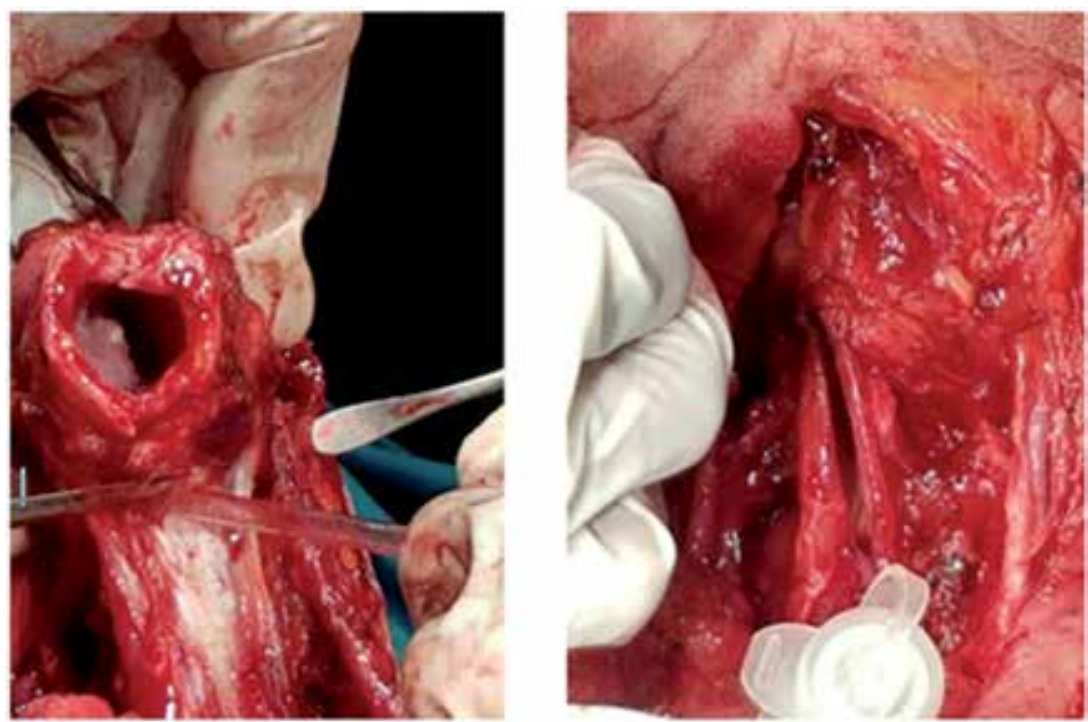

Figure 5.

Reconstruction of the pharynx after total laryngectomy with preservation of the constrictor pharyngeal muscles.

Regarding brachial cysts, lymphangiomas, schwannomas and lipomas their surgical removal implies preservation of neural function. Up to $12 \%$ cases with vagus schwannomas present swallowing disorders prior to surgery. After surgery $85 \%$ of the cases become dysphonic and even develop Horner syndrome. An extensive informed consent of the patient should be obtained before surgery. Also, the phoniatric treatment should begin as soon as possible after surgery [37].

Carotid body and glomus tumors are benign in nature but difficult to treat. Swallowing disorders are rare before surgery and caused by the contact between the tumor and lateral pharyngeal wall. During surgical dissection sometimes is necessary to section the superior laryngeal nerve with diminished swallowing function for 14 days after surgery. Clinical exam reveals a slight paresis of soft palate and vocal cord on the same side. Steroid therapy and supplementary vitamin B are very useful. Food intake should be in small quantity with anterior neck flexion usually without the need for a nasogastric tube [38].

Parapharyngeal tumors have a latent debut of the symptoms. Frequently these are benign tumors but grow in size and compress adjacent structures. Swallowing 


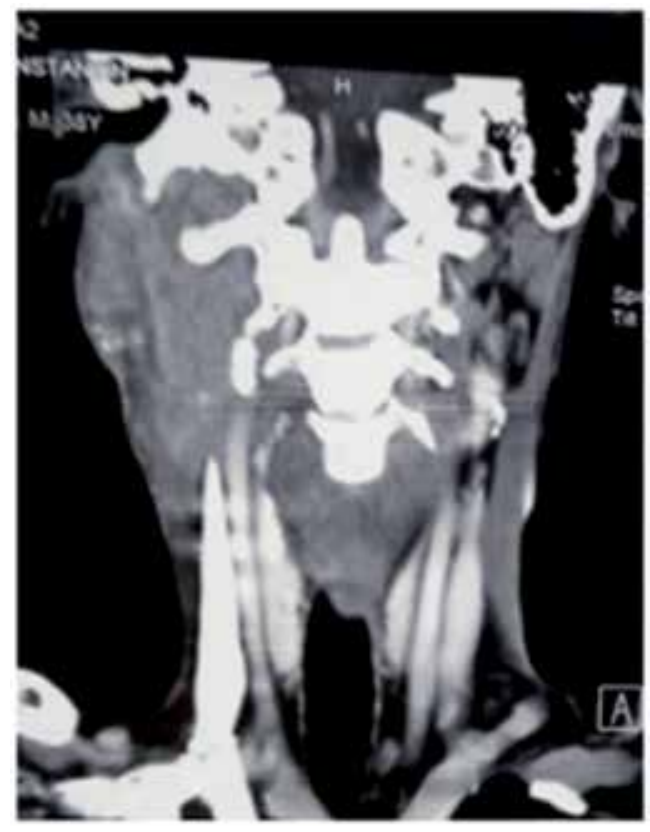

Figure 6.

Metastatic lymph node in the skull base with compression of cranial nerves IX-XII and swallowing disorders.

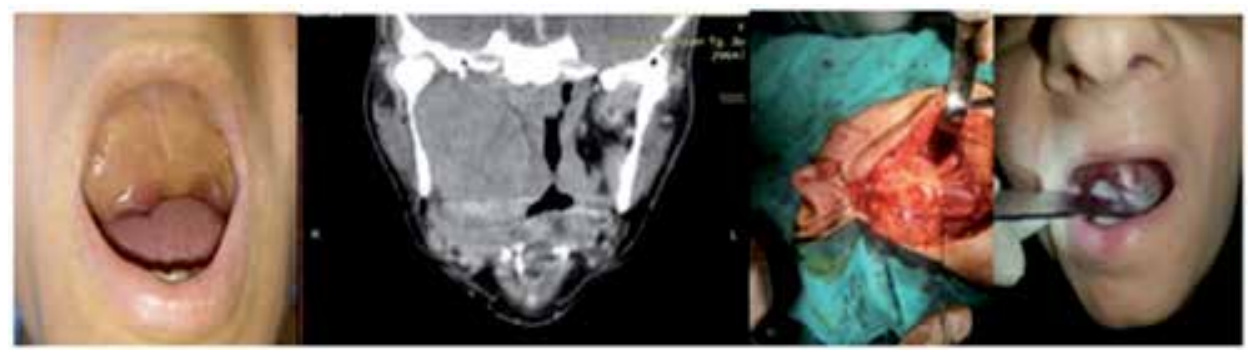

Figure 7.

Parapharyngeal schwannoma with progressive swallowing dysfunction and complete recovery after surgery.

disorders may be the first signs of this pathology. Masses in front of the styloid process grow towards lateral pharyngeal wall between the superior pole of the palatine tonsil and the Eustachian tube. During clinical exam is noted the protrusion of the pharyngeal wall towards the soft palate and sometimes it may be mistaken for a peritonsillar abscess (Figure 7) [39].

Absence of fever and trismus indicates the possibility of a parapharyngeal tumor and requires high resolution CT scan or MRI. During surgery perform careful dissection of the cranial nerves X-XII, the later in close contact with the lateral pharyngeal wall. If this dissection is successful swallowing dysfunctions resolve after local edema retraction in 4 days without further complications [40].

Frequently these tumors are pleiomorphic adenomas of minor salivary glands or derived from the profound lobe of the parotid gland. These tumors evolve behind the styloid process neighboring internal carotid artery, internal jugular vein, cranial nerves IX-XII and cervical sympathetic chain. Swallowing disorders are secondary in these cases and their prognosis depends on the chance of a radical surgical removal and further use of radiation and chemotherapy [41].

Masses from retropharyngeal space are rare. Their approach is transoral if imaging studies tend to support a benign tumor like schwannoma. Swallowing disorders 


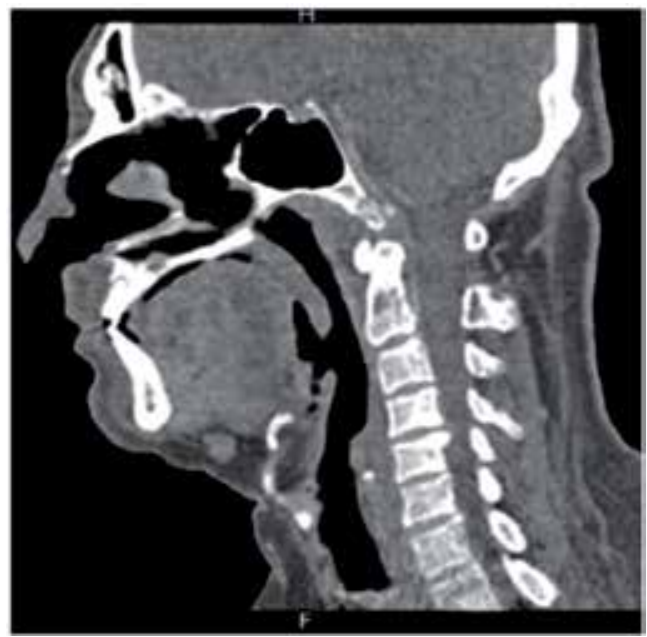

Figure 8.

Metastasis at the level of the skull base on the right side with origin in the kidney and associating paralysis of the right hypoglossal nerve and right recurrent laryngeal nerve with severe swallowing disorders.

usually disappear after tumor ablation. Nasogastric tube should be used till complete healing of the incision on the posterior pharyngeal wall [42].

Skull base tumors produce dysphagia and dysphonia through compression of cranial nerves IX-XII leading to the aforementioned neurology syndromes. Frequently such tumors are metastasis from distant primitive tumors, for example renal malignant tumors (Figure 8). These primitive origin tumors must be documented carefully and should benefit from maximum oncologic therapy according to specific guidelines. Otherwise tackling the secondary distance metastasis is not recommended because of the poor vital prognosis of the patient [43].

\section{Swallowing disorders secondary to oncologic treatment}

During radiation therapy the patient complains of an early onset swallowing difficulty due to oral and pharyngeal mucositis and a latent dysphagia produced by muscular fibrosis. Secondary xerostomia is produced by fibrosis of the salivary glands affected by radiation field and can become permanent [44].

WHO developed a scale for oral mucositis starting from grade 0 without mucositis, first grade with erythema, second grade with ulcers, in grade 3 is necessary to have only a liquid diet and the last stage in which the alimentation is not feasible [45].

\section{Treatment principles for swallowing disorders associated to cervical facial tumors}

The management of these swallowing disorders should focus on three stages: before tumor ablation, during treatment modality and after oncologic treatment. Tumor ablation may be achieved through surgery, radiation or chemotherapy. Before this stage the patient must be prepared to withstand treatment aggression through parenteral nutrition. For optimum nutritional status is also recommended the use of a gastrostomy either classic or PEG. The maximum treatment modality comprises surgical ablation with anatomy reconstruction but not always with great 
functional results. In this stage the use of nasogastric tube is a facile technical solution in order to gain the necessary time for proper pharyngeal sutures healing. At this stage an important aspect the need for an increase uptake in albumin and other proteins to facilitate tissue scaring. The stage after oncologic treatment unfortunately in many cases records the presence of definitive gastrostomy. Palsy of cranial nerves IX-XII will benefit from administering steroids and B vitamins supplements along with phoniatric exercises [46].

There are some practical aspects to analyze starting from the complete informed consent of the patient regarding the functional outcome of cervical facial surgery with targeted oncologic resection. Most often the surgeon is focused on airway complications and tends to neglect the swallowing dysfunctions. There is a shortage of trained specialists for recovery of swallowing disorders. Ideally in every head and neck oncology center there should be a team of specialists focused on early recovery of swallowing and speech dysfunctions [47].

Nutrition after surgery is very important for an optimum physical and psychological recovery of the patient being an important element of the quality of life. The presence or absence of swallowing disorders after surgery is a prognostic factor for the positive evolution of the patient. The evaluation of dysphagia should be part of standard oncology survey. Monitoring the body weight is an indicator of favorable evolution of the patient according to current oncologic nutrition protocols [48].

For recovery after swallowing disorders there are various compensatory strategies: adapting the posture while swallowing with the chin, head rotation or combined movements. For patients with significant tongue resections can be used palatal augmentation or reshaping prosthesis [49].

\section{Conclusions}

The ENT specialist encounters swallowing disorders in cervical facial tumors as an early diagnosis element, an aspect of surgical procedures and surgical healing and a residual symptom after radiotherapy or chemotherapy. History taking is a key element in diagnosis and the specialist should record pain during swallowing, provocation of cough during swallowing, avoiding food with higher consistency, weight loss, prior history of pneumonia and other neurological diseases. Should be very clear the beginning of dysphagia and associated symptoms such as dysphonia and dyspnea. Clinical ENT exam should be thorough with an extensive flexible fiber endoscopy for occult tumors at the level of the rhinopharynx, palatine tonsils, tongue base, and pharyngeal-laryngeal junction. A consistent amount of time should be allotted to cranial nerves exam and complete neurology consults.

For objective evaluation of swallowing disorders in cervical facial tumors the barium transit can be very useful because it facilitates direct dynamic view of the swallowing process and aspiration of the contrast media in the larynx and trachea. In some particular cases, the contrast media should be sterile and soluble for preventing aspiration lesions during examination. It is important to have an objective scale to evaluate swallowing for introduction of parenteral nutrition and monitoring the patient evolution. A subjective evaluation of the impact of swallowing dysfunction on quality of life is made through self-evaluating questionnaires which can be very useful in monitoring the evolution, too.

The management of these swallowing disorders should focus on 3 stages: before tumor ablation, during treatment modality and after oncologic treatment such radiation and chemotherapy. For optimum nutritional status is also recommended to use a gastrostomy classic or PEG. The maximum treatment modality comprises surgical ablation with anatomy reconstruction but not always with great functional 
results. In this stage the use of nasogastric tube is a facile technical solution in order to gain the necessary time for proper pharyngeal sutures healing. Also, an important aspect is the need for an increase uptake of albumin and other proteins to facilitate tissue scaring. A good nutritional status is necessary for a positive prognosis in swallowing disorders. Palsy of cranial nerves IX-XII will benefit from administering steroids and B vitamins supplements along with phoniatric exercises. It is important to make a complete informed consent of the patient regarding the functional outcome of cervical facial surgery.

There is a shortage of trained specialists for recovery of swallowing disorders. Ideally in every head and neck oncology center there should be a team of specialists focused on early recovery of post-therapeutically swallowing and speech disorders. Special exercises should begin as soon as possible after surgery with specific alimentary bolus volumes for every case. The swallowing disorders in cervical facial tumors are an important element for monitoring patient evolution, because a patient without dysphagia after therapy has a good local evolution and also an increased quality of life.

\section{Author details}

Daniela Vrinceanu ${ }^{1 *}$ and Mihai Dumitru ${ }^{1,2}$

1 ENT Department, Emergency University Hospital, Bucharest, Romania

2 Anatomy Department, Carol Davila University of Medicine and Pharmacy,

Bucharest, Romania

*Address all correspondence to: vrinceanudana@yahoo.com

\section{IntechOpen}

(C) 2019 The Author(s). Licensee IntechOpen. This chapter is distributed under the terms of the Creative Commons Attribution License (http://creativecommons.org/licenses/ by/3.0), which permits unrestricted use, distribution, and reproduction in any medium, provided the original work is properly cited. (cc) BY 


\section{References}

[1] Philpott H, Garg M, Tomic D, Balasubramanian S, Sweis R. Dysphagia: Thinking outside the box. World Journal of Gastroenterology. 2017;23(38):69426951. DOI: $10.3748 /$ wjg.v23.i38.6942

[2] Koch W. Dysphagia in oropharyngeal squamous cell carcinoma in perspective. Cancer. 2017;123(16):3003-3004. DOI: $10.1002 /$ cncr.30710

[3] Lango MN, Egleston B, Fang C, Burtness B, Galloway T, Liu J, et al. Baseline health perceptions, dysphagia, and survival in patients with head and neck cancer. Cancer. 2014;120(6):840847. DOI: $10.1002 /$ cncr.28482

[4] Yamauchi T, Edahiro A, Watanabe Y, Murakami M, Satou E, Saito H, et al. Risk factors for postoperative dysphagia in oral cancer. The Bulletin of Tokyo Dental College. 2012;53(2):67-74

[5] Speyer R, Heijnen BJ, Baijens LW, Vrijenhoef FH, Otters EF, Roodenburg N, et al. Quality of life in oncological patients with oropharyngeal dysphagia: Validity and reliability of the Dutch version of the MD Anderson Dysphagia Inventory and the Deglutition Handicap Index. Dysphagia. 2011;26(4):407-414. DOI: 10.1007/ s00455-011-9327-3

[6] Costa MMB. Neural control of swallowing. Arquivos de Gastroenterologia. 2018;55(Suppl 1): 61-75. DOI: 10.1590/S0004-2803. 201800000-45

[7] Zancan M, Luchesi KF, Mituuti CT, Furkim AM. Onset locations of the pharyngeal phase of swallowing: Metaanalysis. CoDAS. 2017;29(2):e20160067. DOI: 10.1590/2317-1782/20172016067

[8] Matsuo K, Palmer JB. Anatomy and physiology of feeding and swallowing: Normal and abnormal. Physical
Medicine and Rehabilitation Clinics of North America. 2008;19(4):691-707, vii. DOI: $10.1016 /$ j.pmr.2008.06.001

[9] Balasubramanian G, Sharma T, Kern M, Mei L, Sanvanson P, Shaker R. Characterization of pharyngeal peristaltic pressure variability during volitional swallowing in healthy individuals. Neurogastroenterology and Motility. 2017;29(11):e13119-e13121.

DOI: $10.1111 / \mathrm{nmo} .13119$

[10] Kagaya H, Yokoyama M, Saitoh E, Kanamori D, Susa C, German RZ, et al. Isolated pharyngeal swallow exists during normal human feeding. The Tohoku Journal of Experimental Medicine. 2015;236(1):39-43. DOI: 10.1620/tjem.236.39

[11] Molfenter SM, Leigh C, Steele CM. Event sequence variability in healthy swallowing: Building on previous findings. Dysphagia. 2014;29(2):234-242. DOI: $10.1007 /$ s00455-013-9501-x

[12] Chen JH. Ineffective esophageal motility and the vagus: Current challenges and future prospects. Clinical and Experimental Gastroenterology. 2016;9:291-299

[13] Jo YR, Chung CW, Lee JS, Park HJ. Vernet syndrome by varicellazoster virus. Annals of Rehabilitation Medicine. 2013;37(3):449-452. DOI: 10.5535/arm.2013.37.3.449

[14] Gutiérrez Ríos R, Castrillo Sanz A, Gil Polo C, Zamora García MI, Morollón Sánchez-Mateos N, Mendoza Rodríguez A. Collet-Sicard syndrome. Neurología. 2015;30(2):130-132. DOI: 10.1016/j.nrl.2013.04.002

[15] Knyazer B, Smolar J, Lazar I, Rosenberg E, Tsumi E, Lifshitz T, et al. Iatrogenic Horner syndrome: 
Etiology, diagnosis and outcomes. The Israel Medical Association Journal. 2017;19(1):34-38

[16] Mumtaz S, Henry A, Singh M. Tapia's syndrome. Anesthesia Progress. 2018;65(2):129-130. DOI: 10.2344/ anpr-65-04-06

[17] Raber-Durlacher JE, Brennan MT, Verdonck-de Leeuw IM, Gibson RJ, Eilers JG, Waltimo T, et al. Swallowing dysfunction in cancer patients. Support Care Cancer. 2012;20(3):433-443. DOI: 10.1007/s00520-011-1342-2

[18] Verdonschot RJCG, Baijens LWJ, Vanbelle S, Florie M, Dijkman R, Leeters IPM, et al. Medically unexplained oropharyngeal dysphagia at the University Hospital ENT outpatient clinic for dysphagia: A cross-sectional cohort study. Dysphagia. 2019;34(1):43-51. DOI: 10.1007/ s00455-018-9912-9

[19] Owen W. ABC of the upper gastrointestinal tract: Dysphagia. BMJ. 2001;323(7317):850-853

[20] Michalopoulos K, Gunasekaran S, Moor JW, Bem C. Dysphagia with a thyroid nodule: Is there a primary aerodigestive malignancy? Journal of the Royal Society of Medicine. 2008;101(3):144-145. DOI: 10.1258/ jrsm.2008.070166

[21] Barbon CE, Steele CM. Efficacy of thickened liquids for eliminating aspiration in head and neck cancer: A systematic review. Otolaryngology and Head and Neck Surgery. 2015;152(2):211-218. DOI: $10.1177 / 0194599814556239$

[22] Dziewas R, Glahn J, Helfer C, Ickenstein G, Keller J, Ledl C, et al. Flexible endoscopic evaluation of swallowing (FEES) for neurogenic dysphagia: Training curriculum of the German Society of Neurology and the
German stroke society. BMC Medical Education. 2016;16:70. DOI: 10.1186/ s12909-016-0587-3

[23] Lee SY, Kim BH, Park YH. Analysis of dysphagia patterns using a modified barium swallowing test following treatment of head and neck cancer. Yonsei Medical Journal. 2015;56(5):1221-1226. DOI: 10.3349/ ymj.2015.56.5.1221

[24] Shune S, Karnell L, Karnell M, Van Daele D, Funk G. Association between severity of dysphagia and survival in patients with head and neck cancer. Head \& Neck. 2012;34:776-784. DOI: 10.1002/hed.21819

[25] Goepfert RP, Lewin JS, Barrow MP, Gunn GB, Fuller CD, Beadle BM, et al. Long-term, prospective performance of the MD Anderson dysphagia inventory in "Low-Intermediate Risk" oropharyngeal carcinoma after intensity modulated radiation therapy. International Journal of Radiation Oncology, Biology, Physics. 2017;97(4):700-708. DOI: 10.1016/j. ijrobp.2016.06.010

[26] Amin MR, Achlatis S, Lazarus CL, Branski RC, Storey P, Praminik B, et al. Dynamic magnetic resonance imaging of the pharynx during deglutition. The Annals of Otology, Rhinology, and Laryngology. 2013;122(3):145-150

[27] Chilukuri P, Odufalu F, Hachem C. Dysphagia. Missouri Medicine. 2018;115(3):206-210

[28] Son YR, Choi KH, Kim TG. Dysphagia in tongue cancer patients. Annals of Rehabilitation Medicine. 2015;39(2):210-217. DOI: 10.5535/ arm.2015.39.2.210

[29] Saud MRM, Salahuddin Z, Hassan A, Yunus MRM, Mohamad I, Zulkifli MM. Laryngeal giant cell tumour presenting as a tongue base 
lesion causing severe dysphagia. Journal of Taibah University Medical Sciences. 2017;13(2):201-204. DOI: 10.1016/j. jtumed.2017.10.002

[30] Grinnell M, Logeman A, Knudsen T, Sayed Z. Deep parotid lobe abscess presenting with dysphagia and trismus. Case Reports in Otolaryngology. 2019;2019:2931015. DOI: $10.1155 / 2019 / 2931015$

[31] Pracy P, Loughran S, Good J, Parmar S, Goranova R. Hypopharyngeal cancer: United Kingdom National Multidisciplinary Guidelines. The Journal of Laryngology and Otology. 2016;130(S2):S104-S110

[32] Vella O, Bequignon A, Comoz F, Babin E. Retropharyngeal liposarcoma: A rare cause of dysphagia. European Annals of Otorhinolaryngology, Head and Neck Diseases. 2016;133(6):429430. DOI: 10.1016/j.anorl.2016.07.006

[33] Short MW, Burgers KG, Fry VT. Esophageal cancer. American Family Physician. 2017;95(1):22-28

[34] Simioniuc A, Anghel AG, Dumitru M, Anghel AO, Anghel I. Left hemilaryngeal carcinoma complicated with an external laryngocele.

Romanian Journal of Medical Practice. 2016;11(1):79-82

[35] Anghel AG, Anghel I, Dumitru M, Anghel AO, Cristian D, Burcos T. The importance of using the nutrition through endoscopic percutaneous gastrostomy in the management of patients with head and neck carcinoma. Romanian Journal of Medical Practice. 2015;10(2):156-163

[36] Vrinceanu D, Dumitru M, Nica AE. ENT private eye for unknown primary metastatic lymph nodes. ORL. ro. 2019;42:20-22

[37] Costache A, Dumitru M, Tweedie D, Sarafoleanu C, Anghel I. Adult cervical
lymphangioma-Ultrasonography, surgical removal, and pathology results. Case report. Medical Ultrasonography. 2015;17(3):410-412

[38] Bali GS, Hartman DJ, Haight JB, Gibson MK. A rare case of malignant glomus tumor of the esophagus. Case Reports in Oncological Medicine. 2013;2013:287078. DOI: $10.1155 / 2013 / 287078$

[39] Vrinceanu D, Dumitru M, Georgescu M. Impact of HPV and other tumoral markers in tonsillar squamous cell carcinoma (SCC) —A retrospective study. ORL.ro. 2018;41(4):12-17. DOI: 10.26416/ORL.41.4.2018

[40] Anghel I, Anghel AG, Soreanu CC, Dumitru M. Tonsilar tumoral pathology, the experience of Coltea ENT clinic. Romanian Journal of Medical Practice. 2012;4(28):320-323

[41] Vrinceanu D, Dumitru M, Stefan A, Banica B, Nica A. In search of a syndrome-A case with synchronous tumors in the parotid gland, thyroid gland and kidney. In: Proceedings of the National ENT, Head and Neck Surgery Conference 2018; Filodiritto; ISBN: 97888-85813-13-7. pp. 530-534. Available from: filodirittoreditore.com

[42] Garcia-OrtegaDY,Gomez-PedrazaA, Luna-Ortiz K. Parapharyngeal space lipomatosis with secondary dyspnea, disphagia and disphonia. International Journal of Surgery Case Reports. 2015;15:54-56. DOI: 10.1016/j. ijscr.2015.08.016

[43] Shakir SI, Pelmus M, Florea A, Boileau JF, Guiot MC, Di Maio S, et al. Synchronous metastatic skull base chordoma to the breast: Case report and literature review. Current Oncology. 2016;23(2):e154-e157. DOI: 10.3747/ co.23.2896

[44] King SN, Dunlap NE, Tennant PA, Pitts T. Pathophysiology of 
radiation-induced dysphagia in head and neck cancer. Dysphagia. 2016;31(3):339-351. DOI: 10.1007/ s00455-016-9710-1

[45] Sonis ST, Elting LS, Keefe D, Peterson DE, Schubert M, HauerJensen M, et al. Perspectives on cancer therapy-induced mucosal injury: Pathogenesis, measurement, epidemiology, and consequences for patients. Cancer. 2004;100(9 Suppl):1995-2025

[46] Anghel AG, Anghel I, Dumitru M, Cristian D, Burcos T. The use of gastrostomy procedures in HNC patients. Chirurgia. 2013;108:341-345

[47] Strojan P, Hutcheson KA, Eisbruch A, Beitler JJ, Langendijk JA, Lee AWM, et al. Treatment of late sequelae after radiotherapy for head and neck cancer. Cancer Treatment Reviews. 2017;59:79-92. DOI: 10.1016/j. ctrv.2017.07.003

[48] Govender R, Smith CH, Taylor SA, Barratt H, Gardner B. Swallowing interventions for the treatment of dysphagia after head and neck cancer: A systematic review of behavioural strategies used to promote patient adherence to swallowing exercises. BMC Cancer. 2017;17(1):43. DOI: 10.1186/ s12885-016-2990-x

[49] Messing BP, Ward EC, Lazarus CL, Kim M, Zhou X, Silinonte J, et al. Prophylactic swallow therapy for patients with head and neck cancer undergoing chemoradiotherapy: A randomized trial. Dysphagia. 2017;32(4):487-500. DOI: $10.1007 /$ s00455-017-9790-6 


\section{Edited by Monjur Ahmed}

The purpose of writing this book is to discuss the updated information on voice and swallowing disorders. The book has been written by international authors and experts in this field. You will find not only clinical aspects but also basic science aspects of voice and swallowing disorders. The chapters include the quantitative analysis of activity patterns of muscles of mastication and deglutition, and salivary secretion after facial massage and vibrotactile stimulation. You will also find the updated management of oropharyngeal dysphagia, dysphagia due to cervical facial tumors, radiation-induced dysphagia, and dysphagia in patients with a stroke. This book can be an important guide to the practicing physicians and surgeons managing voice and swallowing disorders.

\section{IntechOpen}

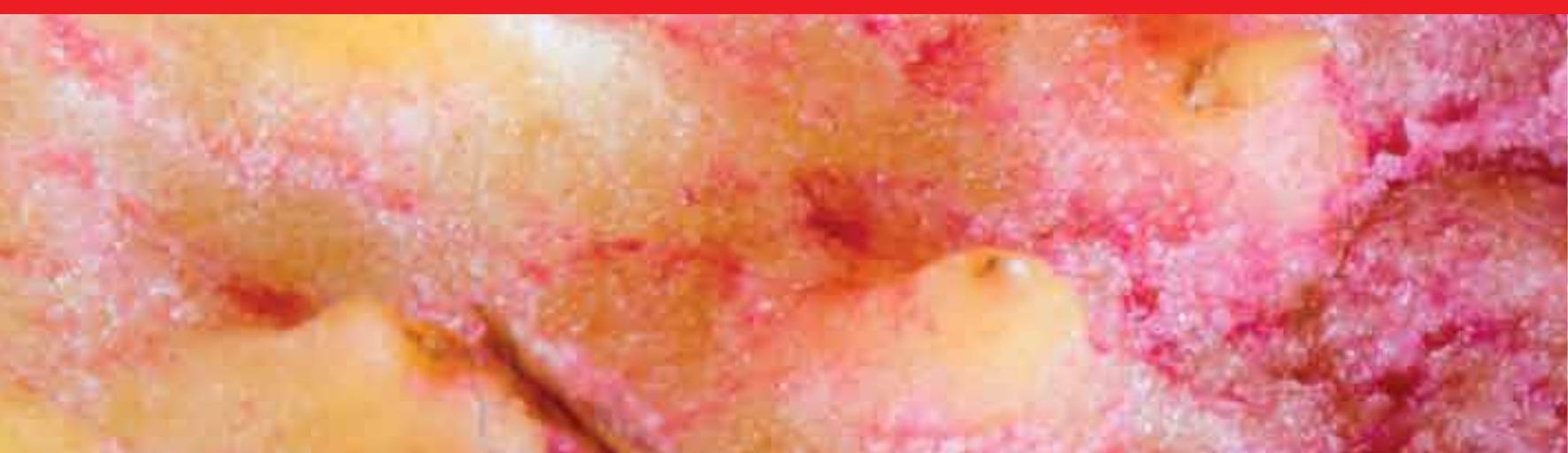

